\title{
STED nanoscopy of synaptic substructures in living mice
}

\section{Dissertation}

for the award of the degree "Doctor rerum naturalium" of the Georg-AugustUniversity Göttingen

within the doctoral program Molecular Physiology of the Brain (CMPB) of the Georg-August-University School of Science (GAUSS)

submitted by

Jennifer-Magdalena Masch

born in Hamburg, Germany 


\section{Members of the Thesis Committee}

Prof. Dr. Stefan W. Hell (Referee)

Department of NanoBiophotonics

Max Planck Institute for Biophysical Chemistry, Göttingen

Prof. Dr. Detlev Schild (2 ${ }^{\text {nd }}$ Referee)

Department of Neurophysiology and Cellular Biophysics

Georg-August-University Göttingen

\section{Prof. Dr. Nils Brose}

Department of Molecular Neurobiology

Max Planck Institute of Experimental Medicine, Göttingen

\section{Further members of the Examination Board}

\section{Prof. Dr. Sarah Köster}

Research Group for Nanoscale Imaging of Cellular Dynamics Institute for X-Ray Physics

Georg-August-University Göttingen

\section{Prof. Dr. Michael Hörner}

Department of Cellular Neurobiology

Johann-Friedrich-Blumenbach-Institute for Zoology and Anthropology

Georg-August-University Göttingen

\section{PD Dr. Alexander Egner}

Department of Optical Nanoscopy

Laser-Laboratorium Göttingen e.V. 
I declare that this thesis was composed by myself and that the work contained herein is my own except where explicitly stated otherwise in the text. Where the work was done in collaboration with others, I have made significant contributions that are clearly indicated throughout the thesis. I further declare that this work has not been submitted for any other degree or professional qualification except as specified. 
The best that most of us can hope to achieve in physics is simply to misunderstand at a deeper level.

Wolfgang Pauli (1958) 


\section{ABSTRACT}

Optical nanoscopy has revolutionized far-field microscopy, enabling the observation of subcellular structures and dynamics from completely new perspectives. Among other fields, neuroscience benefits greatly from the remarkable advances in super-resolution microscopy, which provide unprecedented insights into the molecular organization and function of synapses. STED nanoscopy has been one of the most successful methods for live-cell applications and is the only superresolution technique that has been demonstrated for imaging in living mice so far. However, in vivo nanoscale imaging of synaptic proteins has remained challenging due to the extraordinary complexity of the experiments and the lack of adequate labeling tools. The goal of this thesis was to overcome these challenges and image for the first time the distribution and substructure of a synaptic protein in vivo by STED nanoscopy with markers in the far-red spectral range.

In order to achieve this aim, this work focused on the optimization and integration of three essential elements of in vivo super-resolution imaging: (i) the development of a robust STED nanoscope, (ii) the establishment of a protocol for stable animal preparation, and (iii) the identification of an effective fluorescent labeling approach. First, a compact in vivo two-color STED nanoscope for the far-red spectrum was constructed, which best fulfilled the specific requirements for imaging the brains of living mice. A new design concept with minimized optical beam path lengths was developed, which resulted in maximum optical stability and reduced the need for frequent alignment procedures. In addition, a protocol for surgical preparation of the mice was established with particular emphasis on the mechanical stability of the mouse head fixation. This new procedure of animal preparation considerably reduced imaging artifacts due to cardiac and respiration induced motion. Lastly, highly specific labeling strategies based on far-red fluorophores for live-cell imaging were developed. The performance of various far-red fluorescent proteins and organic dyes was evaluated for in vivo applicability and the dyes 610CP and silicon-rhodamine (SiR) were identified as the most suitable fluorophores for in vivo far-red STED nanoscopy at $775 \mathrm{~nm}$ depletion wavelength.

The synergy of these three essential elements then enabled the first super-resolution study of a synaptic protein in the brains of living mice. The postsynaptic density 95 (PSD-95) protein was examined in a transgenic knock-in mouse line expressing endogenous PSD-95 in fusion with the selflabeling enzyme HaloTag. The results of this study uncovered the native nanoscopic structural organization of PSD-95 in dendritic spines of the visual cortex and revealed complex morphologies which were unresolved by confocal microscopy and not yet reported in such detail in in vitro model systems.

The present work underscores the importance of in vivo super-resolution microscopy and introduces a novel approach for future investigations of the molecular organization and function of the brain and other tissues in living animals. 


\section{ABBREVIATIONS}

\begin{tabular}{|c|c|}
\hline $1 D, 2 D, 3 D$ & one-, two-, three-dimensional \\
\hline AAV & adeno-associated virus \\
\hline ACSF & artificial cerebrospinal fluid \\
\hline AMPA & a-amino-3-hydroxy-5-methyl-4-isoxazolepropionic acid receptor \\
\hline AMPAR & AMPA receptor \\
\hline AOTF & acousto-optic tunable filter \\
\hline APD & avalanche photodiode \\
\hline BP & bandpass filter \\
\hline $\mathrm{CO}_{2}$ & chemical formula for carbon dioxide \\
\hline CW & continuous wave \\
\hline DM & dichroic mirror \\
\hline eGFP & enhanced green fluorescent protein \\
\hline EM & electron microscopy \\
\hline EOM & electro-optic modulator \\
\hline$f$ & focal length \\
\hline F-actin & filamentous actin \\
\hline FP & fluorescent protein \\
\hline FPGA & field-programmable gate array \\
\hline FWHM & full width at half maximum \\
\hline GFP & green fluorescent protein \\
\hline $\mathrm{H}_{2} \mathrm{O}$ & chemical formula for water \\
\hline hSyn & human synapsin promoter \\
\hline ID & inner diameter \\
\hline $1 / 0$ & input/output \\
\hline LP & longpass filter \\
\hline$n$ & refractive index \\
\hline MMF & multimode fiber \\
\hline NA & numerical aperture \\
\hline $\mathrm{NaCl}$ & chemical formula for sodium chloride \\
\hline NMDA & $\mathrm{N}$-methyl-D-aspartate receptor \\
\hline OD & outer diameter \\
\hline PAINT & points-accumulation-for-imaging-in-nanoscale-topography \\
\hline PALM & photoactivation localization microscopy \\
\hline PBS & phosphate-buffered saline \\
\hline PFA & paraformaldehyde \\
\hline PMT & photomultiplier tube \\
\hline PSD & postsynaptic density \\
\hline PSD-95 & postsynaptic density 95 protein \\
\hline PSF & point spread function \\
\hline rAAV & recombinant adeno-associated virus \\
\hline SD & standard deviation \\
\hline SiR & silicon-rhodamine \\
\hline SLM & spatial light modulator \\
\hline SP & shortpass filter \\
\hline STED & stimulated emission depletion \\
\hline STORM & stochastic optical reconstruction microscopy \\
\hline YFP & yellow fluorescent protein \\
\hline
\end{tabular}




\section{CONTENTS}

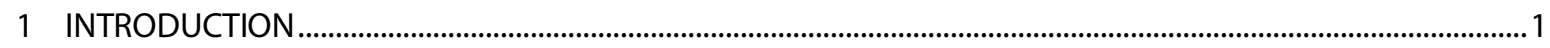

1.1 Far-field optical microscopy...................................................................................................

1.2 Fluorescence-based super-resolution far-field microscopy ....................................................................

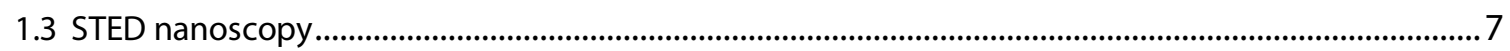

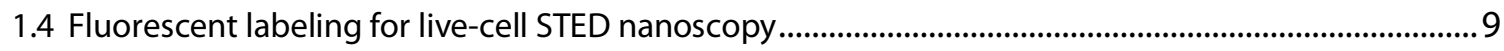

1.5 Super-resolution imaging of postsynaptic proteins...................................................................11

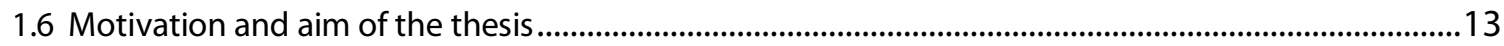

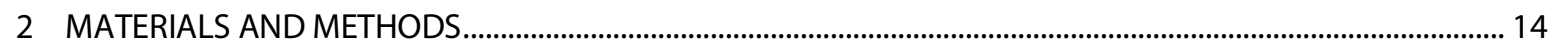

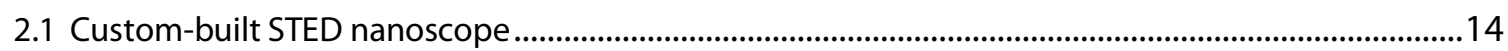

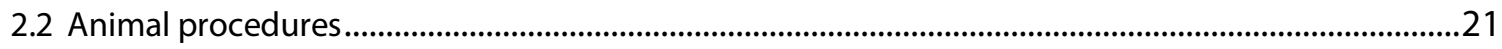

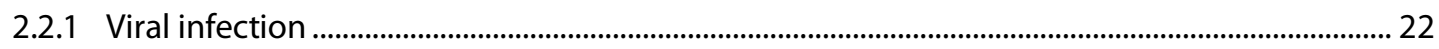

2.2.2 Surgical preparation of the mouse for STED imaging .................................................................... 24

2.2.3 Fluorescent organic dye injection............................................................................................... 27

2.2.4 Preparation and imaging of fixed brain slices ................................................................................. 28

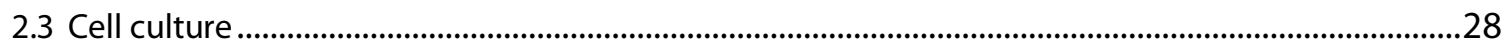

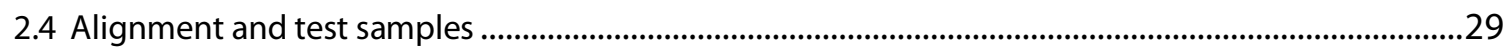

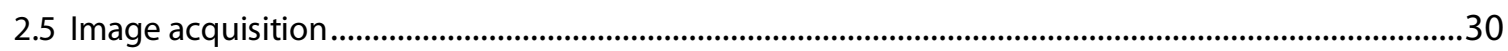

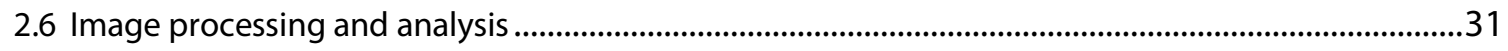

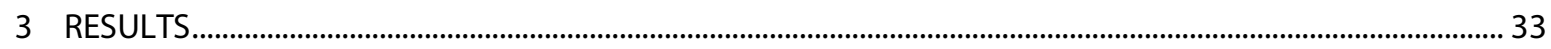

3.1 New design concept for the compact in vivo STED nanoscope ............................................................33

3.2 Performance of the compact STED nanoscope …………..................................................................

3.2.1 Characterization of the PSF ................................................................................................................. 37

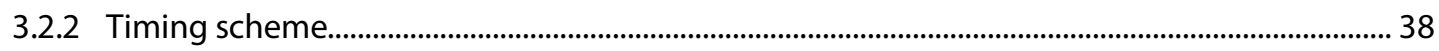

3.2.3 Effective confocal and STED resolution ....................................................................................... 40

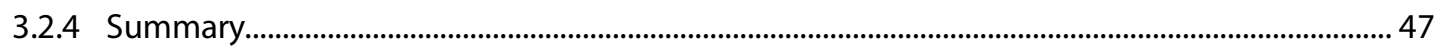

3.3 Development of labeling strategies for in vivo far-red STED imaging.................................................4

3.3.1 Labeling based on far-red fluorescent proteins ............................................................................ 48

3.3.2 Labeling based on far-red fluorescent organic dyes ........................................................................ 53

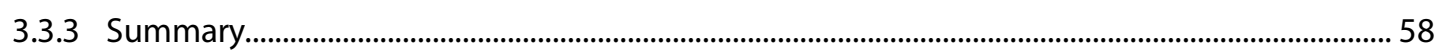

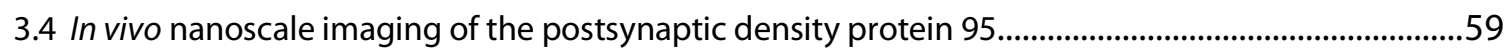

3.4.1 Distribution and nanoscale organization of PSD-95 in living mice ............................................. 59

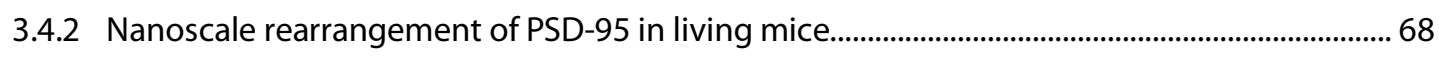

3.4.3 Summary............................................................................................................................ 71

4 CONCLUSIONS AND OUTLOOK ............................................................................................................... 72

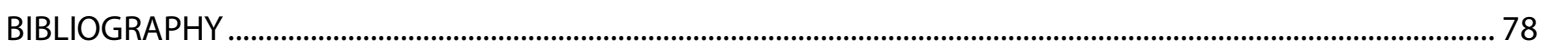

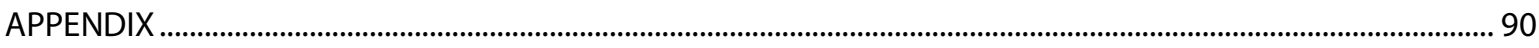

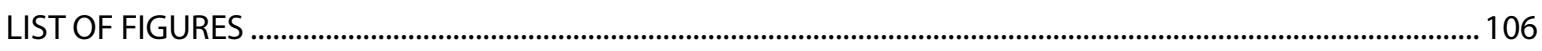

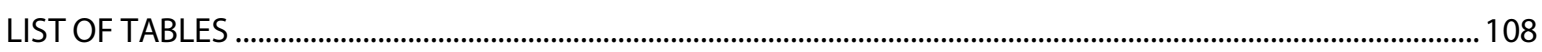

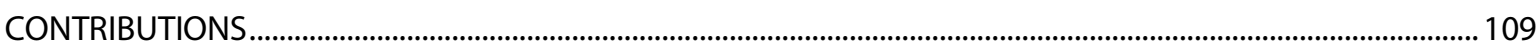

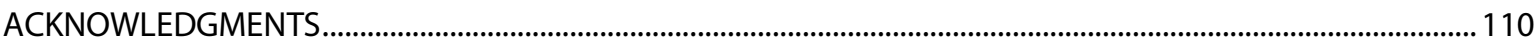




\section{INTRODUCTION}

During the last century, many new discoveries across the natural sciences were made by numerous technological advances, enabling to observe our environment from completely new perspectives. A great variety of applications was facilitated by light microscopy which, together with electron microcopy, proved to be one of the most powerful forms of microscopy. Light microscopy stands out for its ability to image structures minimally invasive, so that living cells can be studied without disturbing their natural environment. By labeling proteins with fluorescent markers, fluorescence microscopy allows to examine their organization, distribution, and interaction with very high specificity and contrast. The advent of fluorescence-based super-resolution far-field microscopy techniques at the end of the $20^{\text {th }}$ century has significantly revolutionized approaches to study organisms on the nanoscale, which have traditionally been accessible only by electron microscopy. Among other fields, neuroscience benefits greatly from super-resolution microscopy unraveling previously unexplored details of neuronal structures and, in particular, of synapses, which are the sites at which information is transmitted from one cell to the other. Synapses are only few folds larger than the diffraction-limit and therefore their substructures cannot be resolved with conventional optical microscopes.

In the following sections, the development of optical microscopy towards super-resolution microscopy is described briefly. Different super-resolution concepts are introduced, emphasizing STimulated Emission Depletion (STED) nanoscopy in particular due to its outstanding ability for in vivo imaging. An overview of live-cell labeling techniques for STED nanoscopy and recent superresolution studies about postsynaptic proteins is given, before the thesis project is introduced at the end of this section.

\subsection{Far-field optical microscopy}

The concept of optical microscopy dates back to the late $16^{\text {th }}$ century, when the first compound microscope was invented by Hans Jansen and his son. The Dutch spectacle-makers arranged two lenses in a tube, enabling the alteration of the magnification by varying the distance between them (reviewed by Clay and Court, 1933; Karlsson Rosenthal, 2009). However, the first microscopic observations were published by Robert Hooke and Antonie van Leeuwenhoek (Hooke, 1665; van Leeuwenhoek, 1673). Both developed their own optical microscopes and began with the studies of living specimen at the micrometer scale. Their microscopes created the fundamental basis for the technology that has become the key instrument in the field of biology, neuroscience, and medicine. The technical development of optical microscopy over the past four centuries was constantly driven by the demand for higher resolution and greater contrast with minimal aberration to get the best images of magnified biological structures. Despite the proper design of microscope lenses and enhanced specimen illumination, the improvement of light microscopy reached its physical limits, as described by Ernst Abbe in 1873 (Abbe, 1873). Abbe reported that light is diffracted when passing through an opening, limiting the spatial resolution of optical microscopes. The resolution is defined as the smallest distance between two nearby point objects, at which the objects still can be distinguished as two separate entities. The so-called diffraction barrier hampers to discern two pointlike objects, which are closer than approximately half the wavelength of the imaging light (Abbe, 1873). 
The minimum resolvable distance $d$ in the lateral $(x, y)$ and axial $(z)$ image plane is defined by the following formulae (von Helmholtz, 1874; Born and Wolf, 1999):

$$
d_{\text {lateral }}=\frac{\lambda}{2 n \cdot \sin (\alpha)}
$$

and

$$
d_{\text {axial }}=\frac{\lambda}{2 n \cdot \sin ^{2}(\alpha / 2)}
$$

where $\lambda$ the wavelength of the used light, $n$ the refractive index of the imaging medium, and $\alpha$ the half-aperture angle of the objective lens. Consequently, by using light of shorter wavelengths and objective lenses with higher numerical apertures $(N A=n \cdot \sin (\alpha))$, the spatial resolution can be improved to a certain finite extent. Thus, conventional optical microscopes theoretically can provide resolutions of $d_{\text {lateral }}=500 \mathrm{~nm} /(2 \cdot 1.4) \approx 180 \mathrm{~nm}$ and $d_{\text {axial }}=500 \mathrm{~nm} /\left(2 \cdot 1.52 \cdot \sin ^{2}\left(67^{\circ} /\right.\right.$ $2)) \approx 540 \mathrm{~nm}$ when operating at $500 \mathrm{~nm}$ wavelength and implementing an oil immersion objective lens with an NA of 1.4. Due to optical aberrations, inhomogeneities in the sample, and noise, the actual resolution is even worse and typically does not reach these theoretical limits. Usually, the point spread function (PSF) is used to characterize the optical performance of a microscope. It describes the response of an imaging system to a point source or point object and includes all aberrations of the optical system. For optical systems with a space invariant PSF, the image is a convolution of the object with the PSF. A common way for determining the resolution of an optical microscope is to measure the PSF, i.e. the response of a single point light source e.g. fluorescent bead (Shaw and Rawlins, 1991).

Since many biological specimens are by nature transparent and contain features with low contrast, several methods have been investigated over the last century to enhance the contrast. Among phase contrast (Zernicke, 1935), differential interference contrast (Nomarski, 1955), and others, fluorescence is used as the most popular contrast mode. Fluorescence is the emission of radiation by a molecule (fluorophore) after excitation with electromagnetic radiation. The molecule features discrete energy levels as shown in the Jablonski diagram (Jabłoński, 1933) in Figure 1.1. Besides the electronic states, it can also reside in a vibrational or rotational state. In thermal equilibrium, the molecule is typically in its singlet ground state $\left(\mathrm{S}_{0}\right)$. By absorption of light with a specific wavelength, the molecule is excited to any vibrational state of the excited singlet state $\left(S_{1}\right)$. The energy difference between the ground state and the excited state is a property of the fluorophore. The excited molecule thermally relaxes within a few picoseconds to the lowest vibrational state of $S_{1}$. The molecule resides typically in this state for a few nanoseconds (fluorescence lifetime). The transition to $S_{0}$ occurs either by emitting a photon (spontaneous emission) or is non-radiative via internal conversion. Since energy is lost in this absorption-emission cycle due to thermal relaxation, the emitted photon has a lower energy compared to the incident photon. Correspondingly, the emission spectrum is red-shifted with respect to the absorption spectrum, referred to as the Stokes shift (Stokes, 1852). Beside the transition from $S_{1}$ to $S_{0}$, the excited molecule can also non-radiatively transit to the excited triplet state $\left(T_{1}\right)$ via intersystem crossing which involves a spin flip of the electron. This transition is formally forbidden, but occurs with a low probability due to strong spinorbit coupling. Because the excited triplet state is usually energetically lower than the excited singlet state, the transition from $T_{n}$ to $S_{n}$ is extremely unlikely, but was reported for some fluorophores (Reindl and Penzkofer, 1996). Returning from $T_{1}$ to $S_{0}$ is also formally forbidden, resulting in a longer lifetime of the triplet state typically in the order of several microseconds compared to the singlet state. The transition to $S_{0}$ occurs either by a photon emission (phosphorescence) or non-radiatively. 


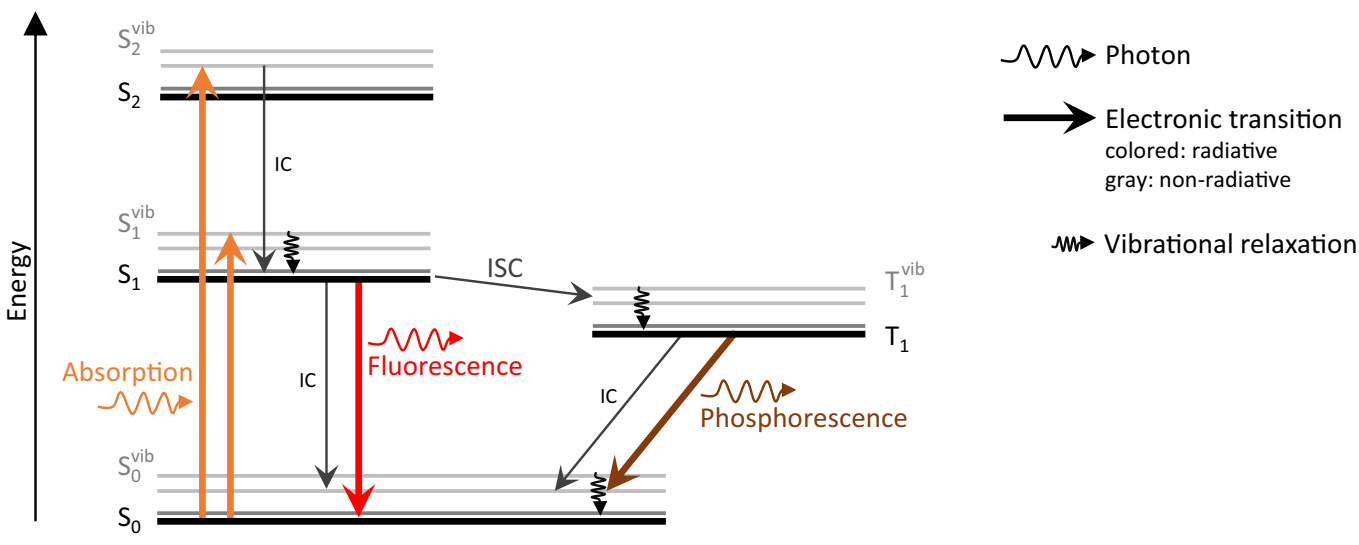

Figure 1.1: Jablonski energy diagram

Different energy levels of a molecule with singlet states $S_{n}$ and triplet state $T_{1}$. Arrows indicate possible transitions between the states (IC: internal conversion, ISC: intersystem crossing).

After the observation of fluorescence reported first by John Herschel (Herschel, 1845) and later by George Stokes (Stokes, 1852), Adolf von Bayer synthesized the first fluorescent dye, fluorescein, in 1871 (Bayer, 1871). At the beginning of the $20^{\text {th }}$ century, Carl Zeiss and Carl Reichert constructed the first fluorescence microscope (Heimstadt, 1911; Reichert, 1911; Lehmann, 1913). Since then, fluorescence microscopy became one of the most influential forms of microscopy due to its excellent contrast mode. Conventional epi-fluorescence microscopes consist of a light source, optical filters and dichroic mirrors, an objective and a tube lens, and a detector (e.g. camera). The specimen is illuminated by the excitation light of a specific wavelength through the objective lens and emits fluorescence. The objective lens collects the fluorescence signal, which is then separated from the excitation light by a dichroic mirror. After spectral filtering to reject the undesired background light, the fluorescence is imaged on a detector by the tube lens.

Over the last century, a large variety of fluorescent labels has been developed. Due to the development of fluorescently-labeled antibodies (Coons et al., 1942) and cloning of the green fluorescent protein (GFP) (Prasher et al., 1992; Chalfie et al., 1994; Heim and Tsien, 1996), fluorescence microscopy has become the method of choice when imaging biological specimen with molecular specificity. Furthermore, the sample preparation is relatively simple and specimens can be imaged under live-cell conditions.

Abbe's theory of microscopic imaging (Abbe, 1873) was regarded as the ultimate resolution limit for a long time. Over the last decades, however, several far-field optical microscopy techniques have been developed to maximize the lateral resolution and to introduce optical sectioning capabilities within the diffraction limit.

A significant milestone has been the investigation of the confocal concept (Minsky, 1961; Minsky, 1988), on which confocal laser scanning fluorescence microscopy is based on. It relies on scanning the sample with focused illumination (laser beam) and detecting the fluorescence signal spatially filtered by a pinhole placed at the image plane of the objective lens (Cremer and Cremer, 1978; Sheppard and Wilson, 1981). In point-scanning confocal microscopy, an image is acquired by moving either the sample or the focused light beam. The detector records the fluorescence signal at each position and the final image is generated by mapping the signal to the corresponding pixel of the image. In contrast to a conventional wide-field fluorescence microscopy, out-of-focus fluorescence signal is eliminated by the pinhole, thereby enabling optical sectioning in axial direction and providing higher image contrast. If the size of the pinhole is infinitely small, theoretically, the size of the image PSF can be sharpened by up to a factor of $\sqrt{2}$ and accordingly increasing the resolution (Wilson and Sheppard, 
1984; Pawley, 2006). However, in practice, only a pinhole with finite size can be implemented because the spatial filter also degrades the amount of detected fluorescence photons (Cox and Sheppard, 2004).

In two- or multiphoton excitation laser scanning fluorescence microscopy, two or multiple photons of lower energy are simultaneously ${ }^{1}$ absorbed by the fluorescent molecule to cause an excitation similar to the absorption of a single photon which has twice or $\mathrm{m}$-fold energy ( $\mathrm{m}$ : number of photons; Göppert-Mayer, 1931; Denk et al., 1990; Zipfel et al., 2003). Since the probability of simultaneous absorption of two or multiple photons is relatively rare, very high photon densities are typically required. Accordingly, the excitation volume is confined to the immediate surrounding of the focal spot, facilitating axial sectioning similar to confocal microscopy. Because a detection pinhole is not necessary, the fluorescence signal can be detected more effective without spatial filtering. Due to non-linear excitation with longer wavelength (typically near-infrared light), the major advantages of multiphoton excitation microscopy are axial sectioning, large depth penetration due to less absorption and scattering, and photobleaching limited to the focal plane (Cox and Sheppard, 2004). These benefits make multiphoton microscopy an attractive approach, in particular, for live-cell and (deep) tissue imaging (Svoboda et al., 1997; Helmchen and Denk, 2005).

The need to further improve the axial resolution led to the development of point-scanning $4 \mathrm{Pi}$ microscopy, which implements two opposing objective lenses focusing onto the same focal spot (Hell and Stelzer, 1992). The sample, positioned between both lenses, is coherently illuminated from both sides, so that the fluorescence occurs from an almost isotropic focal spot. Each objective lens collects the emitted fluorescence, which is superimposed for the reconstruction of the final image. The concept of increasing the effective NA by utilizing two opposing objective lenses is also applied in wide-field image interference microscopy, named as $I^{2} M$ (Gustafsson et al., 1995; Gustafsson et al., 1996). Both imaging modalities are rather complex in instrumentation and image processing, thus only being used in fluorescence microscopy when high axial resolution is desired.

An attempt to enhance the spatial resolution comes from Structured Illumination Microscopy (SIM) that, unlike the previously described techniques, is not based on a point-scanning system. SIM can yield a resolution improvement in both lateral and axial dimensions by illuminating the sample with a structured light pattern, which is generated by interference of multiple excitation beams (Heintzmann and Cremer, 1998; Frohn et al., 2000; Gustafsson, 2000). The interaction of the patterned illumination and the sample causes a shift of high image frequencies, usually not transmitted by the optical system ${ }^{2}$, down to lower ones, resulting in a low-frequency pattern (Moiré pattern). For the reconstruction of the final image, several images are recorded with different orientations of the structured illumination pattern. Because high frequency information of the image is preserved, the spatial resolution is increased by a factor of 2 compared to conventional microscopy.

The above-mentioned far-field optical microscopy techniques, which are by no means exhaustive, have been developed to improve the resolution in lateral and/or axial dimension beyond the limit of conventional microscopy (see Equations 1.1 and 1.2). However, the resolution is still restricted by diffraction. While these imaging techniques focused on instrumental improvements to increase the spatial resolution, in the early 1990s a groundbreaking concept was developed to overcome the diffraction barrier.

\footnotetext{
${ }^{1}$ In spatially and temporally coincidence

${ }^{2}$ The aperture of a lens filters the spatial frequencies of the image such that only frequencies below a certain cutoff frequency pass.
} 


\subsection{Fluorescence-based super-resolution far-field microscopy}

The fundamental advance for breaking the diffraction limit in optical microscopy was the insight of exploiting the photophysical and photochemical properties of the fluorophore itself, enabling theoretically unlimited spatial resolution in the far-field (Hell, 1994; Hell and Wichmann, 1994; Betzig, 1995; Hell and Kroug, 1995; Hell, 2007). By taking advantage of the transitions between distinguishable states of the fluorophore, the origin of the fluorescence signal can be localized much more precisely than the extent of the imaging PSF. The fluorescent molecule typically exists in a fluorescent (on) and a non-fluorescent (off) state (Hell, 2009). In general, the transition between states is the key factor responsible for discerning adjacent fluorescent molecules or features with diffraction-unlimited precision, i.e. no longer limited by the used wavelength or numerical aperture of the objective lens. Nowadays, several imaging concepts can provide resolutions on the nanoscale (actually molecular-size), and are collectively referred to as super-resolution microscopy or nanoscopy (Hell, 2007; Eggeling et al., 2015). While all super-resolution concepts are based on fluorescent state transitions, they differ in the way how they control the fluorescence emission for image acquisition. They are typically classified into coordinate-targeted and coordinate-stochastic super-resolution modalities as well as combinations thereof.

\section{Coordinate-targeted super-resolution approach}

The underlying concept of coordinate-targeted nanoscopy methods is based on REversible Saturable Optical Linear Fluorescence Transitions (RESOLFT, Hell, 2003). The fluorescence emission is confined to a targeted position with a size below the diffraction limit. This is achieved by illuminating the sample with a light pattern featuring isolated points of ideally zero intensity, which spatially controls the respective transitions of neighboring fluorescent molecules. To obtain images with diffraction-unlimited resolution, this light pattern is scanned over the sample and adjacent objects are discerned by sequentially switching their fluorophores on and off.

STimulated Emission Depletion (STED) microscopy was the first imaging method which overcame the diffraction barrier by applying a coordinate-targeted switching and readout scheme (Hell and Wichmann, 1994; Klar and Hell, 1999; Klar et al., 2000). A similar concept (with different switching mechanisms) is used in Ground State Depletion (GSD) microscopy (Hell and Kroug, 1995), Saturated Pattern Excitation Microscopy (SPEM; Heintzmann et al., 2002), Saturated Structured Illumination Microscopy (SSIM; Gustafsson, 2005), and RESOLFT microscopy with reversibly photoswitchable proteins or organic dyes (Hofmann et al., 2005; Bossi et al., 2006).

\section{Coordinate-stochastic super-resolution approach}

Instead of applying an illumination pattern, coordinate-stochastic nanoscopy methods use the switching and localization of single molecules in a wide-field microscopy implementation to generate an image with diffraction-unlimited precision. These methods are based on photoswitchable fluorophores, which provide the required on/off-state transitions spontaneously. The positions of single fluorescent molecules within a focal region can be determined by first isolating them based on their photophysical and photochemical properties, and then by calculating the centroid localizations of the diffraction-blurred image spots (Betzig, 1995). For separation, most molecules remain in a non-fluorescent state, while some fluorophores stochastically switch to the on-state by wide-field illumination (low activation intensity). Once a fluorophore has been activated, it can be excited and the fluorescence signal is recorded by a camera in a wide-field configuration. Subsequently, the activated molecules are switched off (e.g. by photobleaching) and another sparse set of isolated molecules is again stochastically switched to the on-state. This acquisition process is 
repeated until ideally all molecules are localized at least once. A diffraction-unlimited image is reconstructed from thousands to ten-thousands of individual wide-field images, which sequentially recorded the positions of individual, spatially separated (further apart than the diffraction-limited spot size) molecules.

This concept has been initially realized in (Fluorescence) PhotoActivation Localization Microscopy ((F)PALM; Betzig et al., 2006; Hess et al., 2006) and (direct) STochastic Optical Reconstruction Microscopy ((d)STORM; Rust et al., 2006; Heilemann et al., 2008). Further nanoscopy methods using stochastic switching of fluorophores are Points-Accumulation-for-Imaging-in-Nanoscale-Topography (PAINT; Sharonov and Hochstrasser, 2006), and Ground State Depletion followed by Individual Molecule return microscopy (GSDIM; Fölling et al., 2008).

\section{MINFLUX approach}

More recently, a new concept has been introduced that combines aspects of coordinate-targeted and coordinate-stochastic super-resolution approaches to localize fluorescent molecules in time and space with increased photon efficiency. It is based on localizing a single activated fluorescent emitter by probing its fluorescence with a sequential series of patterned excitation beams, featuring displaced local intensity minima of excitation light (Balzarotti et al., 2017). This new approach, called MINimal emission FLUXes (MINFLUX), can be used for both nanoscopy and single-molecule tracking (SMT) applications. For nanoscopy, individual fluorescent molecules are randomly switched on and off, and excited by the set of patterned excitation beams to localize their positions in space. In SMT applications, the position of a single, activated fluorophore is tracked by the set of patterned excitation beams. MINFLUX has demonstrated molecular resolution attaining $\sim 1 \mathrm{~nm}$ precision, resolving molecules only $6 \mathrm{~nm}$ apart (Balzarotti et al., 2017). Owing to its novelty, future developments and investigations intend to observe the structure, distribution, and dynamics of molecules in mammalian cells, applications in living organisms are also conceivable.

Both the coordinate-targeted and coordinate-stochastic nanoscopy methods have achieved spatial resolutions far beyond the limit of diffraction. Due to their difference in operating principle, they have distinct benefits and disadvantages, depending on the specific application.

The main advantage of stochastic methods is their experimental simplicity, requiring little technical effort for their wide-field setups. In contrast to targeted methods, no illumination pattern or special optics are required to achieve super-resolution images. However, stochastic approaches are restricted to photoswitchable fluorophores, limiting the choice of fluorescent proteins or organic dyes. Moreover, they usually require a reducing buffer, which is not live-cell compatible. In addition, the data acquisition time is considerably longer than for targeted approaches, and largely depends on the recording speed of the detection camera and the brightness of the fluorescent label. Both the requirement for special fluorophores and the long recording speeds are limiting the capability of stochastic nanoscopy methods for imaging in living organisms.

In this regard, STED nanoscopy, the most widely implemented coordinate-targeted approach, stands out because of its high temporal resolution and live-cell compatibility. In addition, STED nanoscopy offers optical sectioning based on the implemented confocal detection. In recent years, studies with STED nanoscopy have unraveled previously unresolved details inside living cells, tissue, or organisms (e.g. Willig et al., 2006; Nägerl et al., 2008; Westphal et al., 2008; Urban et al., 2011; Berning et al., 2012; Willig et al., 2014; D'Este et al., 2015; Schneider et al., 2015; Bottanelli et al., 2016). Since STED nanoscopy has been one of the most successful methods for live-cell applications and already demonstrated its potential for imaging in living mice, we applied in vivo STED nanoscopy to study a synaptic protein in its native environment in the context of this thesis. 


\subsection{STED nanoscopy}

In STED nanoscopy, the fluorescence emission is confined to a targeted spot of sub-diffraction size by transferring neighboring fluorescent molecules within the diffraction-limited spot into the ground singlet state $S_{0}$ (non-fluorescent) and into the first excited singlet state $S_{1}$ (fluorescent), respectively. To spatially control the respective transitions of the fluorophores, two laser beams are used for illumination of the sample. While the excitation laser beam transfers the fluorescent molecules from $S_{0}$ to $S_{1}$, the transition of the fluorophores from $S_{1}$ to $S_{0}$ is induced by the depletion laser beam through the process of stimulated emission. Both switching mechanism are illustrated in Figure 1.2a.

(a)

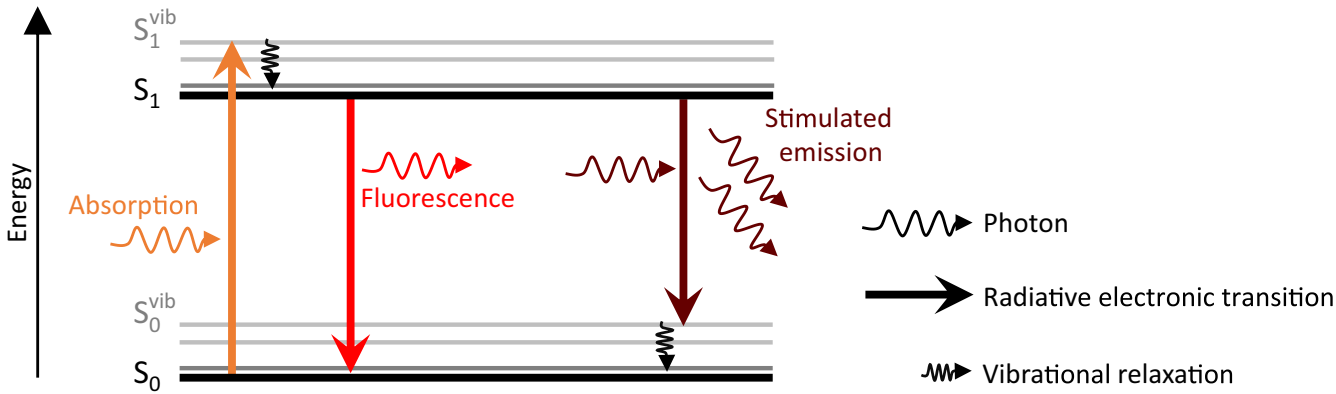

(b)
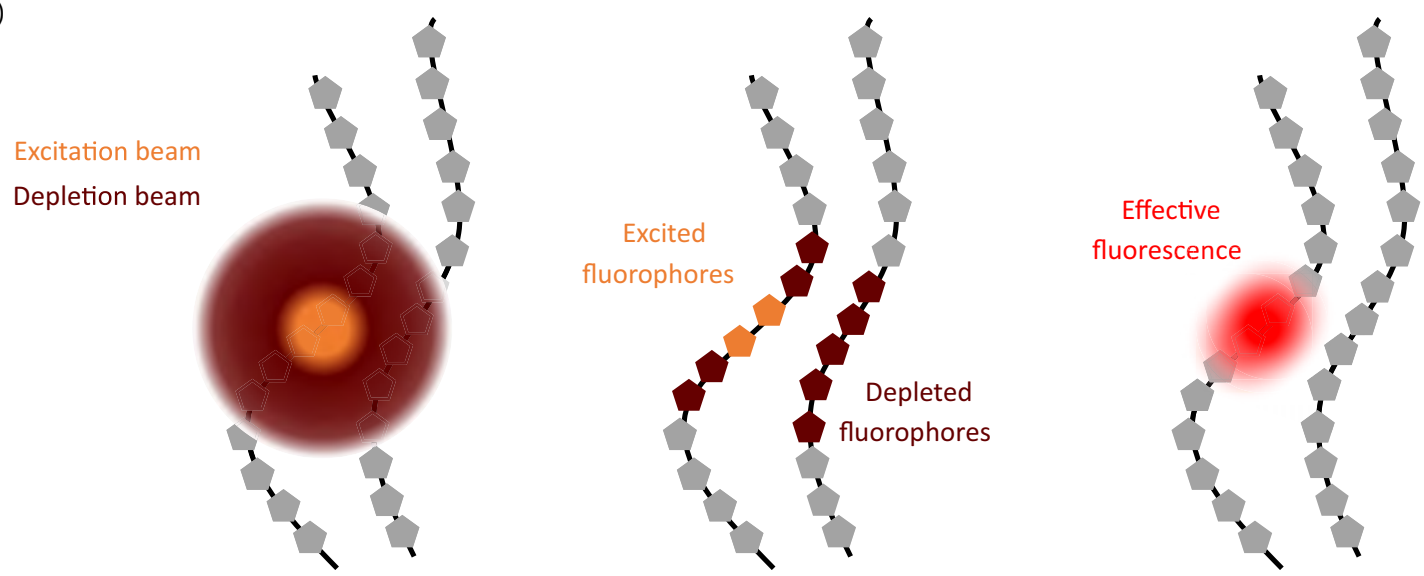

Figure 1.2: Principle of STED nanoscopy

(a) Jablonski diagram illustrating the processes of excitation, fluorescence, and stimulated emission. (b) A sample consisting of two filaments is labeled with fluorophores (gray pentagons). The sample is illuminated with the excitation and the doughnut-shaped depletion laser beams (left panel). Consequently, most of the excited fluorophores in the focal spot are depleted through stimulated emission (middle panel), resulting in a fluorescence signal only from fluorophores that are in the narrow sub-diffraction sized central spot (right panel).

In detail, in each excitation cycle, the fluorescent molecule absorbs a photon of the excitation light, resulting in an electronic transition from the ground singlet state $S_{0}$ to the first excited singlet state $\mathrm{S}_{1}$ (process of absorption). After internal vibrational relaxations within a few picoseconds, the excited molecule spontaneously decays back to the ground state by emitting a red-shifted fluorescence photon (process of spontaneous emission). Alternatively, while the excited molecule is residing in the excited state, the electronic transition from $S_{1}$ to $S_{0}$ can be stimulated by an external photon, causing the emission of a photon with the same wavelength, phase, polarization and propagation direction as the incident photon (process of stimulated emission; Einstein, 1917). 
In STED nanoscopy, off-switching of the excited molecules through stimulated emission is achieved by the depletion beam, which has a red-shifted emission wavelength relative to the excitation laser and is usually located at the redder edge of the fluorescence emission spectrum to avoid undesired re-excitation of the fluorophores to $S_{1}$. Typically, and in this thesis, the depletion beam has a doughnut-shaped intensity profile with an ideally zero intensity at the center to improve the lateral resolution. Axial or three-dimensional enhancement can be achieved by different depletion patterns (Klar et al., 2000; Keller et al., 2007). The doughnut-shaped illumination pattern $\hat{I}_{\text {STED }}$ close to the center can then be approximated by a parabola (Westphal and Hell, 2005; Harke et al., 2008):

$$
\hat{I}_{\text {STED }}(r) \cong 4 I_{\text {STED }} a^{2} r^{2}
$$

where $I_{\text {STED }}$ is the maximal depletion light intensity of the illumination pattern, $a$ the pattern steepness, and $r$ the distance from the center of the focus. By using this depletion illumination pattern, excited fluorophores located off the center of the excitation beam are switched off, and spontaneous fluorescence only occurs from the narrow, sub-diffraction sized central spot (Figure 1.2b). The final image is acquired by scanning the sample with the two superimposed laser beams, measuring the fluorescence signal at each position, and mapping the signal to the corresponding pixel of the image. While the spatial pattern of the depletion laser beam is still diffraction limited, super-resolution is achieved by using a high depletion laser intensity so that the non-fluorescent state transition becomes saturated. The remaining population in the fluorescent state is hereby nonlinear dependent on the depletion laser intensity. The achievable lateral resolution in STED nanoscopy can be approximated by (Westphal and Hell, 2005; Harke et al., 2008):

$$
d_{\mathrm{STED}} \cong \frac{d_{\mathrm{conf}}}{\sqrt{1+I_{\mathrm{STED}} / I_{\mathrm{sat}}}}
$$

where $d_{\text {conf }}$ is the diffraction-limited confocal resolution. $I_{\text {sat }}$ is the saturation intensity of the fluorophore, defining the depletion light intensity at which half of the fluorescence emission is suppressed by stimulated emission. It is dependent on the photophysical and photochemical properties of the fluorescent marker as well as on the wavelength used for the depletion. The STED resolution formula has been formulated using intensities and dependence on the ratio of $I_{\text {STED }} / I_{\text {sat }}$. This ratio can also be expressed by depletion laser powers:

$$
\frac{I_{\text {STED }}}{I_{\text {sat }}}=\frac{P_{\text {STED }}}{P_{\text {sat }}}
$$

with $P_{\text {STED }}=\int_{x y} I_{\text {STED }} \mathrm{d} x \mathrm{~d} y$ and suitably chosen saturation power $P_{\text {sat. }}$. Equation 1.4 can then also be written as:

$$
d_{\mathrm{STED}} \cong \frac{d_{\mathrm{conf}}}{\sqrt{1+P_{\mathrm{STED}} / P_{\mathrm{sat}}}}
$$

where $P_{\text {STED }}$ is the applied depletion laser power. For simplicity, the resolution formula based on laser powers (see Equation 1.6) is used in this thesis.

According to Equations 1.4 and 1.6, the lateral resolution in STED nanoscopy is theoretically unlimited and can be adjusted by the applied intensity/power of the depletion beam. Assuming excellent photostability of the fluorophore, resolution of the size of a single molecule is achievable with sufficiently high depletion laser intensity. Extremely photostable fluorescent nitrogen-vacancy 
centers in diamonds have provided a resolution down to $3 \mathrm{~nm}$ (Wildanger et al., 2012). In practice, however, resolutions are significantly lower due to less photostable fluorophores, which do not tolerate a high amount of the depletion laser intensity being applied. An equally important factor limiting the achievable resolution is the sample itself, which can induce optical aberrations and shall not be photodamaged by high laser exposures. Therefore, resolutions of about $20-30 \mathrm{~nm}$ for samples immuno-labeled with organic fluorophores (Donnert et al., 2006; Göttfert et al., 2013) and about 50-75 nm using genetically expressed fluorescent proteins (Willig et al., 2006; Hein et al., 2008; Morozova et al., 2010; Rankin et al., 2011; Hense et al., 2015; Matela et al., 2017) have been demonstrated.

Since its first experimental realization (Klar and Hell, 1999), the design and implementation of the STED technique have been continuously developed further to improve the performance in terms of image resolution and contrast, data acquisition speed, multicolor imaging, and setup simplicity. The following is by no means exhaustive, but highlights some major advancements. The use of compact, high power fiber lasers with a fixed single wavelength and pico- or nanosecond pulse lengths has had a significant impact for pulsed STED nanoscopes. It has not only reduced the cost and the size of the setups, but has also enhanced the image contrast due to less photobleaching (Göttfert et al., 2013). The implementation of supercontinuum fiber lasers in STED setups has enabled to flexibly generate laser pulses at almost any color for the excitation and depletion from the same spectrally broad-band light source. It has thus simplified multicolor STED imaging and pulse synchronization (Wildanger et al., 2008; Bückers et al., 2011; Osseforth et al., 2014). Implementations with the easySTED phase plate (Reuss et al., 2010) or a spatial light modulator (Booth et al., 2015) for creating the depletion beam pattern have made the microscopes simpler or more versatile. Considerable improvement in imaging speeds has been achieved by the use of fast electro-optical scanners (Schneider et al., 2015), or by parallelized scanning with multiple beams (Bingen et al., 2011; Bergermann et al., 2015). STED imaging with nanometer resolution in all three spatial dimensions has also been demonstrated (Schmidt et al., 2008; Wildanger et al., 2009; Osseforth et al., 2014; Curdt et al., 2015). Using multilevel STED or hyperspectral detection, three- or four-color STED nanoscopy has been considerably improved its flexibility (Sidenstein et al., 2016; Winter et al., 2017). Moreover, implementations such as RESCue-STED (Staudt et al., 2011), protected STED (Danzl et al., 2016), multilevel STED (Sidenstein et al., 2016), MINFIELD-STED (Göttfert et al., 2017), and DyMIN (Heine et al., 2017) have significantly reduced the overall light dose and photobleaching, one of the main limiting factors in STED nanoscopy.

\subsection{Fluorescent labeling for live-cell STED nanoscopy}

Along with reliable instrumentation, the fluorescent labeling of the target structure considerably influences the achievable resolution in STED images and therefore the success of the experiment (Fornasiero and Opazo, 2015). The ideal labeling involves both a bright and photostable fluorophore with finely tuned properties, matching the common specification of the super-resolution systems ${ }^{3}$, as well as a labeling strategy to tag proteins in living cells homogenously with high specificity, nontoxicity, and non-interference with intracellular functions. Over the last decades, a variety of fluorescent markers has been developed for conventional fluorescence microscopy, from which many also have successfully been applied in STED nanoscopy. Different types of fluorophores

\footnotetext{
${ }^{3}$ Among others, the fluorophore has to ensure adequate emission at the available depletion wavelengths (592/660/775 $\mathrm{nm}$ in commercially available systems).
} 
spanning over the whole visible spectrum range are available, but not all of them are applicable for live-cell studies. In general, live-cell compatible fluorescent markers can be divided into two classes: (i) genetically encoded markers (e.g. fluorescent proteins (FPs)) and (ii) non-genetically encoded markers (e.g. organic small-molecule fluorophores, quantum dot, toxins) (Fernandez-Suarez and Ting, 2008; Fornasiero and Opazo, 2015).

The most common genetically encoded markers are FPs ( $25 \mathrm{kD}$ in size) that can be expressed in fusion with a protein of interest and enable the labeling of living cells or organisms with absolute specificity (Chalfie et al., 1994; Ehrenberg, 2008; reviewed in Day and Davidson, 2009; Kremers et al., 2011; Cranfill et al., 2016). The color variety of FPs extends over the entire visible spectrum, whereby the green-yellow variants are considerably brighter than the blue and red ones, which have both a reduced extinction coefficient and a decreased fluorescence quantum yield (Shaner et al., 2005; Day and Davidson, 2009). However, red and far-red FPs are favored in experiments with living tissue or organisms because red-shifted excitation light features lower phototoxicity and increased tissue penetration depth, and the autofluorescence is reduced in the red and far-red spectral range (Nienhaus and Nienhaus, 2014). In general, however, FPs are less photostable than organic dyes, making STED nanoscopy with FPs more difficult. Still, several studies successfully used STED on GFP (Rankin et al., 2011; Tønnesen et al., 2011; Bethge et al., 2013) and its yellow variants (yellow fluorescent protein (YFP), Citrine; Hein et al., 2008; Nägerl et al., 2008; Tønnesen et al., 2011; Urban et al., 2011; Berning et al., 2012; Bethge et al., 2013; Willig et al., 2014), while only three far-red FPs tagRFP657, mGarnet, and mGarnet2 - have been reported to perform well in combination with STED imaging (Morozova et al., 2010; Hense et al., 2015; Matela et al., 2017).

In contrast, non-genetically encoded markers can provide better performances in terms of brightness and enhanced photostability. Among them, organic fluorophores stand out for their size of $<1 \mathrm{kD}$ (Giepmans et al., 2006; Yan and Bruchez, 2015), the possibility of coupling them to the molecule of interest, and the availability of a wide range of spectral options. Nevertheless, specific labeling with organic fluorescent dyes for live-cell imaging experiments requires a certain design for each target (Fernandez-Suarez and Ting, 2008). As an example, fluorophores were fused to lipids and cholesterol to track their diffusion on the cellular membrane (Müller et al., 2011; Saka et al., 2014), bound to toxins for studying the distribution of channels (Massensini et al., 2002; Ondrus et al., 2012; Kuzmenkov et al., 2016), conjugated to antibodies to detect extracellular proteins (Borgdorff and Choquet, 2002; Westphal et al., 2008; D'Este et al., 2015), or, in more recent papers, coupled to moieties targeting intracellular structures (actin, tubulin, DNA, lysosomes and mitochondria; Lukinavičius et al., 2014; Lukinavičius et al., 2015; Lukinavičius et al., 2016; Butkevich et al., 2017).

Self-labeling enzymes (20-30 kDa in size) combine the molecular specificity provided by genetically encoded markers with the benefits of organic fluorophores and represent a versatile approach to enable specific intracellular labeling in living cells (Yan and Bruchez, 2015). The structure of interest is genetically expressed in fusion with an engineered enzyme-tag, which reacts with a membranepermeable substrate leading to the formation of a covalent bond between the tag and the (fluorescent) substrate (Fernandez-Suarez and Ting, 2008; Xue et al., 2015). The most common selflabeling enzymes are SNAP-tag (Keppler et al., 2003), HaloTag (Los et al., 2008), and Clip-tag (Gautier et al., 2008). In the specific case of HaloTag, the protein-tag is a modified haloalkane dehalogenase designed to covalently bind to synthetic ligands (HaloTag-ligands) (Los and Wood, 2007; Los et al., 2008). Several membrane-permeable synthetic ligands are available, and they comprise a chloroalkane linker attached to a fluorescent dye. One of the biggest advantages of self-labeling enzymes is the fact that the fluorescent dyes can be easily changed depending on the specific needs. Several membrane-permeable, bright, and photostable red and far-red substrates are now available, and their use has already been demonstrated in single- or multi-color STED experiments 
(Lukinavičius et al., 2013; Erdmann et al., 2014; Lukinavičius et al., 2014; D'Este et al., 2015; Bottanelli et al., 2016; Butkevich et al., 2016; Lukinavičius et al., 2016; Butkevich et al., 2017). Finally, transgenic knock-in mice carrying an endogenous protein with a SNAP-tag are viable, fertile, and do not exhibit obvious defects (Yang et al., 2015), and in vivo labeling (combined to ex vivo imaging) is specific and homogenous (Grimm et al., 2017).

\subsection{Super-resolution imaging of postsynaptic proteins}

Attempts to elucidate the structural and functional connectivity of the brain have been a major research focus in neuroscience for several decades. About 86 billion neurons form a dense network in the human brain (Azevedo et al., 2009), being connected via specialized junctions called synapses, which are supposed to be the smallest information-processing units of the central nervous system (Sheng and Hoogenraad, 2007). The communication between neurons relies on the morphology and on the molecular organization at the pre- and postsynaptic sites of these connections (Hussain and Huganir, 2016). At most excitatory synapses, dendritic spines along the dendrites are the postsynaptic targets for the presynaptic input from axons (Nimchinsky et al., 2002). These small membranous protrusions comprise a head, which is connected to the dendritic shaft through a neck (Hering and Sheng, 2001) and contains the postsynaptic density ${ }^{4}$ (PSD) (Sheng and Hoogenraad, 2007). The PSD assembles neurotransmitter receptors, cytoskeleton and scaffolding proteins, and signal transduction components into an organized structure (Kim and Sheng, 2004; Sheng and Hoogenraad, 2007), which is assumed to play a decisive role in regulating the strength and structure of the synapse (synaptic plasticity) (Beiique and Andrade, 2003). One of the best-studied and most abundant component of the PSD is the scaffolding protein postsynaptic density 95 (PSD-95) (Chen et al., 2005; Sugiyama et al., 2005; Cheng et al., 2006; Sheng and Hoogenraad, 2007). It anchors neurotransmitter receptors (e.g. AMPA, NMDA) and other postsynaptic membrane proteins, interacts with AMPA receptors (AMPAR) through transmembrane AMPA receptor regulatory proteins (TARPs), and stabilizes numerous signaling molecules within the PSD (Niethammer et al., 1996; O'Brien et al., 1998; Chen et al., 2000; Kim and Sheng, 2004; Nicoll et al., 2006; Huganir and Nicoll, 2013). For a long time, electron microscopy (EM) was the most convenient method to study the morphology of synapses and their molecular organization accomplished by immuno-gold labeling (Peters et al., 1991; Arthur et al., 2007; Chen et al., 2008). However, EM studies are limited by both the labeling approach and the number of synapses that can be studied, and are not possible in living cells. Imaging by light microscopy, on the other hand, allows to study a larger number of synapses, but lacks the resolution to identify molecular features of the synapses due to their submicron size. By breaking the diffraction limit in optical microscopy (see Section 1.2), super-resolution microscopy has provided a complementary approach to resolve the substructure of synapses on the nanometerscale with specific molecular recognition (in an intact environment) (reviewed in Sigrist and Sabatini, 2012; Maglione and Sigrist, 2013; Tønnesen and Nägerl, 2013; Willig and Barrantes, 2014).

\footnotetext{
${ }^{4}$ The PSD was discovered as a region of thickening and increased density close to the postsynaptic membrane by electron microscopy in neurons (Palay, 1956; Palay, 1958; Gray, 1959). Subsequent EM studies have shown that the PSD extends about 30 to $50 \mathrm{~nm}$ from the cytoplasmic surface into the cytoplasm with a diameter of 200 to $500 \mathrm{~nm}$ (Carlin et al., 1980; Gulley and Reese, 1981). The molecular composition of the densely packed PSD was quantified with over 1000 proteins by proteomic analysis (Bayés et al., 2011).
} 
Several super-resolution studies in the recent years have investigated the molecular architecture of the postsynapse and have revealed an organization of proteins in nanodomains (Frank and Grant, 2017). One of the first super-resolution studies of postsynaptic proteins was the characterization of the distribution and organization of acetylcholine receptor supramolecular aggregates in fixed nonneuronal stably expressing Chinese hamster ovary cells (Kellner et al., 2007). STED nanoscopy revealed these neurotransmitter receptors as nanoclusters with a size of $55 \mathrm{~nm}$ and identified a cholesterol dependence of the nanocluster organization. Later, a comprehensive study used multicolor three-dimensional STORM to map the spatial organization of several pre- and postsynaptic proteins in 10-12 $\mu \mathrm{m}$ thick fixed mouse brain cryosections (Dani et al., 2010). Using this approach, the distributions and axial positions of several proteins within individual synapses and with respect to the synaptic cleft were determined. It was reported that PSD-95 is located $\sim 26 \mathrm{~nm}$ away from the synaptic cleft. A more recent study used STED and PALM to analyze the organization of PSD-95 in about 100,000 synapses in hippocampal sub-regions of paraformaldehyde-fixed brain sections (Broadhead et al., 2016). This comprehensive survey revealed that PSD-95 is spatially organized into single- and multi-nanoclusters, which form the basic structural unit of excitatory synapses. The mean size of a nanocluster was determined to be around $145 \mathrm{~nm}$. Moreover, based on the extensive data set, the authors presented a large structural diversity (different numbers of nanoclusters) between synapses of different hippocampal sub-regions. Another recent study used multicolor STORM to investigate the collective arrangement of pre- and postsynaptic nanodomains (pre: RIM1/2, Munc13, and bassoon; post: PSD-95) in fixed cultured rat hippocampal neurons (Tang et al., 2016). This approach has revealed gradients of protein densities, suggesting that nanodomains of pre- and postsynapse are trans-synaptically aligned.

Besides the structural organization, super-resolution techniques have also examined functional processes of postsynaptic proteins. In particular, the single-molecule information provided by coordinate-stochastic nanoscopy methods can be used to track motion of single molecules in spines and to study dynamic changes of nanocluster organizations. Here, several studies concentrated on AMPAR trafficking (Choquet, 2010). Using universal PAINT (uPAINT), Giannone et al., 2010 studied AMPAR mobility in cultured hippocampal neurons and mapped individual AMPAR trajectories in a single spine. Using PALM, MacGillavry and co-workers measured the structural organization and mobility of four postsynaptic scaffolding proteins (including PSD-95) with respect to AMPAR in living neurons with a resolution of $25 \mathrm{~nm}$ (MacGillavry et al., 2013). Their approach identified that the scaffold molecules are concentrated in one or two nanoclusters, which are preferentially enriched in AMPARs. They also revealed time-dependent variations in the spatial distribution of the nanocluster. In a comparative study, four super-resolution techniques (single-particle tracking PALM, UPAINT, STED, and ASTORM) were used to investigate the organization of AMPAR in live and fixed hippocampal neurons (Nair et al., 2013). All modalities demonstrated a comparable organization of AMPARs in nanodomains, which are heterogeneously distributed within the synapses. Often, these nanodomains colocalized with PSD-95 cluster, whereby AMPAR nanodomains $(80 \mathrm{~nm}, 120 \mathrm{~nm}$, and $70 \mathrm{~nm}$ for sptPALM, STED, and UPAINT, respectively) were smaller in size than the PSD-95 cluster.

The vast majority of these super-resolution studies on the PSD was carried out on neurons cultured in conditions that usually inhibit the growth of glial cells (Kaech and Banker, 2006). However, these cells, astrocytes and microglia in particular, regulate the formation, function and elimination of synaptic sites (Chung et al., 2015; Chung et al., 2015; Papouin et al., 2017). Therefore, our current understanding of the organization of the PSD might not reflect its structure within the context of a living brain. 


\subsection{Motivation and aim of the thesis}

Several super-resolution imaging studies have provided new insights into the neuronal composition and function of the synapse, and successfully demonstrated their potential in the field of neuroscience. The majority of studies investigated synapse organization and function in vitro, primarily on fixed cultured neurons or brain slices. However, these model systems might not reflect the native physiological conditions and complexity within the intact brain of a living animal (Lichtman and Denk, 2011; Sigrist and Sabatini, 2012) raising the question whether these observations are also valid in vivo. To date, only two studies demonstrated super-resolution in the cortex of a living mouse, both used the YFP and STED nanoscopy at $\sim 590 \mathrm{~nm}$. The first examined in nanoscopic detail the shape of dendritic spines and its morphological changes (Berning et al., 2012), the second the spatial arrangement of filamentous actin in dendrites and dendritic spines (Willig et al., 2014). Since both studies investigated rather large structural components and unraveled dynamic dendritic spine morphology in vivo, imaging the organization and function of synaptic proteins in the living brain is of particular interest.

We opted not to utilize the green and yellow FPs, because their use increases the risk of tissue damage through laser-induced phototoxic effects. Indeed, in both previous in vivo STED studies, a slight swelling was reported for thicker dendritic morphologies (Berning et al., 2012; Willig et al., 2014). On the contrary, far-red fluorophores are more suitable for live-cell imaging, since the far-red excitation light has lower photon energy and the far-red spectral range lacks common natural absorbers in living tissue (Svoboda and Block, 1994; König, 2000; Weissleder, 2001), leading to lower photodamage. In addition, imaging in the far-red spectral range benefits from the reduced scattering and thus increased light penetration in tissue, and improved image contrast due to lower autofluorescence (Helmchen and Denk, 2005; Xue et al., 2015). Therefore, the goal of this thesis is to image for the first time the distribution and substructure of a synaptic protein in vivo by STED nanoscopy with markers in the far-red spectral range.

In order to achieve this goal, several challenges have to be overcome. First, in vivo imaging requires a robust STED nanoscope. This work aims to develop a new design concept with minimized optical beam path lengths, in order to ensure highest stability and reduce the need for frequent beam alignment. Second, nanoscopic in vivo imaging is likely to suffer from artifacts due to cardiac and respiration induced motion. We strive to minimize these motion artifacts by optimizing the surgical preparation of the mice, with particular emphasis on the mechanical stability of the mouse head fixation. Third, recently developed far-red fluorescent probes for live-cell imaging have not yet been used for in vivo (STED) imaging. Consequently, this work intends to identify the most suitable far-red markers, which enable in vivo labeling with high specificity, sufficient brightness, appropriate photostability and, most importantly, STED compatibility at $775 \mathrm{~nm}$ depletion wavelength. By combining optimized STED nanoscopy, mouse preparation, and far-red labeling, we are able to perform the first super-resolution study of a synaptic protein in its native environment of a living mouse. 


\section{MATERIALS AND METHODS}

Reliable imaging of nanoscopic structures in vivo requires several key elements to work in harmony: a robust (STED) nanoscope, stable animal preparation, and efficient fluorescent labeling. In order to achieve high robustness of the setup in terms of minimal vibrations and alignment requirements, we designed a compact STED nanoscope with minimized optical beam path lengths and with heavy mechanical parts. Moreover, we optimized the mouse preparation to prevent imaging artifacts due to cardiac and respiration induced motion. Even the slightest movements may cause displacements which would be clearly visible in super-resolution images. Therefore, the reduction of motion artifacts was our primary concern when designing and constructing the in vivo STED nanoscope as well as when designing the mouse preparation. In the following sections, the required procedures are described which enable reliable super-resolution STED imaging in living mouse with far-red fluorophores.

\subsection{Custom-built STED nanoscope}

For super-resolving synaptic structures in living, anesthetized mouse with far-red fluorescent markers, we designed and constructed a dual-color STED nanoscope for the far-red spectrum with specific requirements for in vivo experiments. Most prominently, the STED nanoscope was built in an upright configuration to accommodate the physiological orientation of the mouse head. Moreover, adequate space was provided between the objective lens of the microscope and the translation stage to allow the positioning of the mouse and required mouse support equipment.

Figure 2.1, A.1, and A.2 show the design of the dual-color STED nanoscope, which was constructed in a modular fashion (depletion module, excitation module, STED unit, detection module) around a commercial microscope stand (DM6000 FS, Leica Microsystems GmbH, Wetzlar, Germany). The microscope stand not only offered a highly stable configuration of the optical components but also facilitated wide-field observation of large regions of the sample by using the oculars or a sidemounted charge-coupled device camera (DFK23U274, The Imaging Source Europe GmbH, Bremen, Germany). Bright-field as well as fluorescence wide-field imaging were enabled by focusing light from an external fluorescence light source (EL 6000, Leica Microsystems) onto the sample. The commercial filter cube L5 (bandpass filter (BP) 480/40, dichroic mirror (DM) 505, BP 527/30, Leica Microsystems) or a customized filter cube APH HC (BP 600/37, DM 635, BP 675/67, AHF analysentechnik AG, Tübingen, Germany) were used for blue excitation with green detection or for red excitation with far-red detection, respectively. The module that allowed laser-scanning microscopy, dubbed STED unit, was attached on top of the microscope stand on the second port of the microscope tube. A slidable mirror in the microscope stand enabled switching between wide-field and laser-scanning mode. The fixed sample stage supplied by the manufacturer was discarded in favor of a heavy, robust, and stepper motor driven $x y z$-translation stage (380FM-U with customized motorized, height adjustable bridge, Luigs und Neumann Feinmechanik + Elektrotechnik GmbH, Ratingen, Germany), which was independently assembled around the microscope stand (Figure A.1 and A.2). The translation stage features a large base with enhanced stability. This was especially beneficial for maintaining stability when additional equipment e.g. a heating plate to maintain body core temperature of the animal was required to perform the in vivo experiments. The stage offered submicron precise repositioning of the sample over a dimension of $25 \mathrm{~mm} \times 25 \mathrm{~mm} \times 30 \mathrm{~mm}$ (xyz) with 
minimized thermal drift and excellent mechanical stability. The three motor-driven axes of the stage could be controlled remotely (SM7 together with control box SM8, Luigs und Neumann).

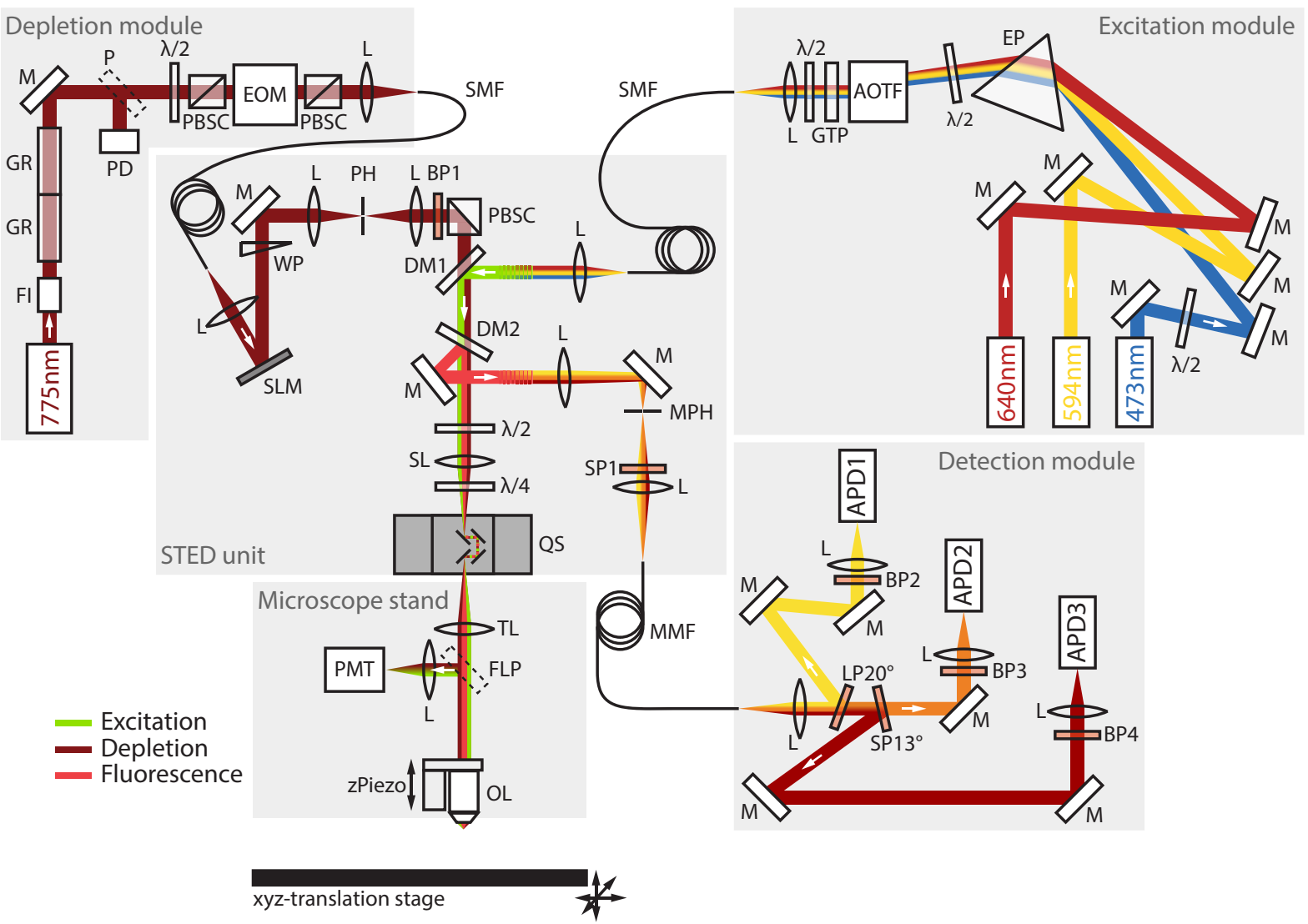

Figure 2.1: Scheme of the custom-built dual-color far-red STED nanoscope for in vivo mouse imaging

$\lambda / 2$ : half-wave plate, $\lambda / 4$ : quarter-wave plate, AOTF: acousto-optic tunable filter, APD: avalanche photo diode, BP: bandpass filter, DM: dichroic mirror, EOM: electro-optic modulator, EP: dispersive equilateral prism, FI: Faraday isolator, FLP: flippable pellicle beam splitter, GR: glass rod, GTP: Glan-Thompson prism, L: lens, M: mirror, LP: longpass filter, MMF: multi-mode optical fiber, MPH: motorized pinhole, OL: objective lens, P: pellicle beam splitter, PBSC: polarizing beam splitter cube, PD: photodiode, PH: pinhole, PMT: photomultiplier tube, QS: Quadscanner, SL: scanning lens, SLM: spatial light modulator, SMF: polarization-maintaining single-mode optical fiber, SP: shortpass filter, TL: tube lens, WP: wedge prism, zPiezo: piezoelectric transducer.

In the following section, the modules (depletion module, excitation module, STED unit, and detection module) are described separately.

\section{Depletion module}

A mode-locked Titanium-Sapphire laser (Mai Tai XF-W1S, Spectra-Physics, Inc., Santa Clara, California, USA) with a repetition rate of $\sim 80 \mathrm{MHz}$ delivered laser pulses with a bandwidth of 5-10 nm in a wavelength range between $710 \mathrm{~nm}$ and $950 \mathrm{~nm}$. For the depletion of the excited fluorophores, the laser was tuned to a wavelength of $775 \mathrm{~nm}$, at which it emitted ultrashort pulses of $80 \mathrm{fs}$ length with an average output power of $1.9 \mathrm{~W}$. After guiding the laser output through a Faraday isolator (IO-5BB800-HP, Thorlabs, Inc., Newton, New Jersey, USA) to prevent back-reflections into the laser cavity, the depletion beam passed two $19 \mathrm{~cm}$ long, highly dispersive SF6 glass rods (home-manufactured) to stretch the pulses to avoid fiber damage. For laser power adjustment and fast on/off-switching, an electro-optic modulator (EOM, LM 0202 P VIS, Linos AG, Göttingen, Germany) was integrated before the laser beam was coupled into a $100 \mathrm{~m}$ long polarization-maintaining single-mode optical fiber (PM630-HP, Thorlabs). The combination of two glass rods and the long single-mode fiber stretched the femtosecond pulses to about 300 ps to avoid two-photon excitation in the sample and to reduce 
photobleaching of the fluorophores. A fast photodiode with a constant fraction discriminator (homebuilt) was triggered by a reflection of the pulsed depletion laser beam from a pellicle (just before the beam passed through the EOM) to measure the arrival time of the depletion laser pulses. This temporal information was used to generate the trigger signal for the excitation lasers as well as for the detection timing.

\section{Excitation module}

For the excitation of the fluorescent markers, one continuous wave (CW) laser (473 nm $100 \mathrm{~mW}$, 06MLD, Cobolt AB, Solna, Sweden) and two pulsed lasers (594 nm $200 \mu W$, custom-built (Henrich, 2014); $640 \mathrm{~nm} 2 \mathrm{~mW}$, LDH-D-C-640, PicoQuant, Berlin, Germany) with pulse lengths $\leq 130 \mathrm{ps}$ at $80 \mathrm{MHz}$ repetition rate were installed. The CW laser with its continuous emission did not need to be synchronized with the depletion laser, whereas synchronization was required for the pulses from excitation and depletion lasers. The timing between the (594 and $640 \mathrm{~nm}$ ) excitation laser pulses and the depletion laser pulses were adjusted by a variable electronic delay (see below) using the optical trigger signal from the depletion laser as the reference. All three excitation laser beams were coupled into a two meter long polarization-maintaining single-mode optical fiber (PMC-460-3.0-NA012-3APC-200-P, Schäfter+Kirchhoff GmbH, Hamburg, Germany) by using a combination of a dispersive equilateral prism (PS859, Thorlabs) and an acousto-optic tunable filter (AOTF, AOTFnc-VIS-TN, AA Opto-Electronic, Orsay, France). Moreover, the AOTF was used to enable fast switching in the microsecond range and laser power control.

\section{STED unit}

The depletion beam emerging from the fiber connected to the depletion module was collimated and guided onto a liquid-crystal based spatial light modulator (LCOS-SLM) (X10468-12(X), Hamamatsu Photonics K.K., Hamamatsu, Japan) at an angle ${ }^{5}$ of $8^{\circ}$. The SLM, placed in a conjugated plane to the pupil of the objective lens, applied a helical phase profile $(0-2 \pi)$ to the wavefront of the depletion beam, resulting in a doughnut-shaped beam profile with a central singularity in the focal plane. Besides flatness correction for the slightly uneven liquid-crystal (correction pattern was provided by the manufacturer), a blazed grating with $0.23 \mathrm{~mm}$ groove period was superposed to the helical phase pattern on the SLM to diffract the phase-modified beam into the first diffraction order. Residual diffraction orders, spatially separated from the first diffraction order, were not required for imaging and were blocked by an aperture (see below) because they did not carry the processed wavefront. Due to the certain bandwidth of the depletion beam, its spectral components were angularly dispersed upon being diffracted at the blazed grating. A wedge prism with an angular deviation of $6^{\circ}$ (PS812-B, Thorlabs), positioned after the SLM, compensated this spectral dispersion by introducing the opposite angular dispersion. This compensation was necessary to avoid a blurred doughnut-shaped intensity pattern in the focal plane. The depletion light was then focused by an achromatic lens onto a pinhole with $100 \mu \mathrm{m}$ diameter to spatially separate the undesired zero order from the first diffraction order of the depletion beam. The pinhole was positioned in the Fourier plane to allow only the first-order light to pass through. The beam was subsequently re-collimated by another achromatic lens. A bandpass filter (BP1, ET780/40m, Chroma Technology Corp., Bellows Falls, Vermont, USA) and a polarizing beam splitter cube (PBS102, Thorlabs) were used to clean up the spectral width and the polarization of the depletion beam. It was then co-axially combined with the excitation beams emerging from the fiber coming from the excitation module via a dichroic

\footnotetext{
${ }^{5}$ Without the tilt, the SLM would deflect the beam back into its incoming propagation direction. Thus, the SLM was slightly tilted to deflect the phase-modulated beam away from the incoming propagation direction.
} 
mirror (DM1, T680 LPXXR, Chroma). The co-aligned depletion and excitation beams passed through a half-wave plate ( $\lambda / 2$, RAC 4.2.10 o.F., Bernhard Halle Nachfolger GmbH, Berlin, Germany) and a quarter-wave plate ( $\lambda / 4$, RAC 4.4.10 o.F., B. Halle) to ensure circular polarization of all laser beams. Scanning the sample in both lateral directions with a maximum field-of-view of $130 \mu \mathrm{m} \times 130 \mu \mathrm{m}$ was performed by a home-built beam scanner (named Quadscanner, Engelhardt, 2010; Görlitz et al., 2014) consisting of four galvanometric mirrors. The scanner was mounted on the top-located cmount of the microscope stand, and the intermediate image plane was placed at the center of the scanner mirrors. The orientation of the galvanometric mirrors decoupled the position and the angle of the beam for both scanning axes and induced less aberrations compared to conventional twomirror beam scanning concepts (Bingen et al., 2011). The achromatic lens ( $f=100 \mathrm{~mm}$ VIS, G05201 5000, Qioptiq Photonics GmbH \& Co. KG, Göttingen, Germany) in front of the beam scanner served as a scanning lens and formed an intermediate image within the pairs of the scanner mirrors. The intermediate image was captured by a tube lens $(f=200 \mathrm{~mm}$, Leica Microsystems) to the pupil of a $63 \times$ glycerol-immersion objective lens (NA=1.30, $n=1.46$; HC PL APO 63×/1.30 GLYC CORR CS2, Leica Microsystems), mounted on a closed-loop piezoelectric microscope lens positioner (zPiezo, MIPOS 100 PL CAP M25, piezosystem jena $\mathrm{GmbH}$, Jena, Germany) for fine $z$ positioning with a range of $100 \mu \mathrm{m}$. The objective lens featured a correction collar to compensate the spherical aberrations due to refractive index mismatch, or variations of cover glass thickness or temperature. $A$ small piezoelectric rotary motor (Piezo LR17 with PMD 101 driver, PiezoMotor Uppsala AB, Uppsala, Sweden) was mounted onto the objective lens to enable extremely precise motorization of the correction collar with low vibrations. An $80 \%$ glycerol/20\% water mixture (type G, Leica Microsystems) with a refractive index of $n=1.45$ at room temperature $\left(21^{\circ} \mathrm{C}\right)$ was used as the immersion medium. Typically, the correction collar was adjusted for the coverslips with \#1.5 thickness. Besides the high NA glycerol-immersion objective lens for imaging small regions, a 10X objective lens (HC PL S-APO 10x/0.30, Leica Microsystems) was integrated into the objective changer of the microscope stand to allow larger field-of-view observation of the sample for coarse orientation.

The fluorescence emitted from the sample was collected by the objective lens, descanned by passing back through the beam scanner, and finally separated from the depletion and excitation laser beams by using a quad-bandpass dichroic mirror (DM2, custom-made, ZT473/594/640/745-800tpc-UF3, Chroma). To eliminate the out-of-focus light, the object plane was imaged onto a motorized pinhole (Variable Pinhole TCS 2, Leica Microsystems) with variable sizes between $20 \mu \mathrm{m}$ and $600 \mu \mathrm{m}$. Typically, the pinhole size was set to a diameter of $50 \mu \mathrm{m}$ and its backprojected size corresponds to 1.2 Airy units of the fluorescence wavelength of $685 \mathrm{~nm}$. To reject stray depletion laser light and avoid Ramaneffects, a shortpass filter (SP1, ET720 SP-2P, Chroma) was integrated before the fluorescence was coupled into a two meter long multimode fiber (MMF, FG050LGA-Custom-Muc, Thorlabs) with a core diameter of $50 \mu \mathrm{m}$.

Besides the confocal detection path, the STED nanoscope also contained a photomultiplier tube (PMT), which served as a non-confocal detection element for the reflected light from the sample (e.g. from gold nanobeads). The backscattered light was coupled out from the common beam path by a pellicle beam splitter (BP545B1, Thorlabs), which was integrated in a home-built filter cube. By flipping the pellicle cube into the beam path, the reflected light was laterally coupled out of the microscope stand at the filter cube wheel and focused onto the sensor of the PMT (H10721-20, Hamamatsu). 


\section{Detection module}

The fluorescence emerging from the MMF was collimated and two optical filters (LP20 ${ }^{\circ}$, longpass filter at $20^{\circ}$ angle of incidence, BLP01-594R-25, Semrock, Inc., Rochester, New York, USA; SP13 shortpass filter at $13^{\circ}$ angle of incidence, FF01-655/SP-25, Semrock) split the fluorescence into three detection channels (green-yellow: $497-553^{6} \mathrm{~nm}$; red: $611-635^{6} \mathrm{~nm}$; dark-red: $653-717^{6} \mathrm{~nm}$; Figure 2.2). Both filters were angle-tuned in order to enable precise discrimination between the red and the dark-red detection channel as well as to facilitate maximum transmission of the fluorescence signal in the dedicated detection channel. For each detection channel, a BP was integrated to reject reflected excitation and depletion laser light (green-yellow: BP2, FF03-525/50-25, Semrock; red: BP3, FF01-623/24-25, Semrock; dark-red: BP4, ET685/70m, Chroma). After spectral filtering, the fluorescence was focused onto a free-space avalanche photodiode (APD) (SPCM-ACRH-13, Excelitas Technologies Corp., Waltham, Massachusetts, USA).

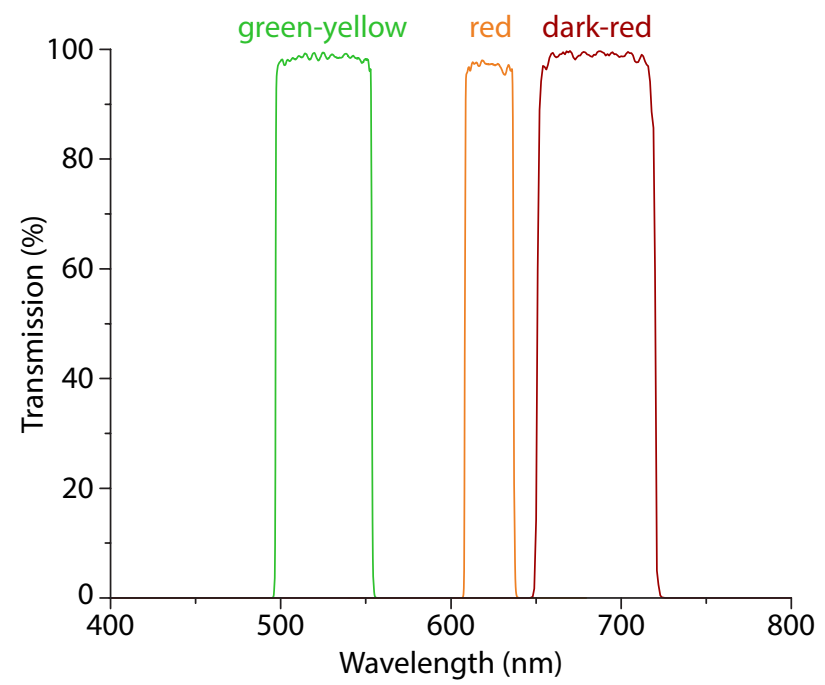

Figure 2.2: Spectral ranges of the three detection channels

A combination of a $594 \mathrm{~nm} \mathrm{LP}$ and a $655 \mathrm{~nm} \mathrm{SP}$, both angle-tuned, was used to separate the fluorescence signal into three detection bands (green-yellow: $497-553^{6} \mathrm{~nm}$; red: $611-635^{6} \mathrm{~nm}$; dark-red: $653-717^{6} \mathrm{~nm}$ ).

Each APD counted the detected fluorescence photons. The single photon events in the APD modules were translated into transistor-transistor-logic (TTL) pulses, which were forwarded to counters in the field-programmable gate array (FPGA) electronics (see below). These counters were gated synchronously to the trigger signal of the depletion laser. Time-gated detection was applied to eliminate the early part of the fluorescence signal, which was spontaneously emitted by the fluorophores either before or during the depletion pulse.

\section{Electronic control- and signal pathways}

For the hardware control of the STED nanoscope, we used an FPGA unit consisting of three main components: an FPGA board (PCle-7852R, National Instruments Corp., Austin, Texas, USA), a commercial input/output (I/O) breakout box (NI SCB-68A, National Instruments), and a custom-built I/O connector, dubbed fast signal connector (FSC). Major functions of the FPGA unit were laser-, scanning-, and detection-control as well as image data acquisition and transfer to the $\mathrm{PC}$ via PCI bus. Figure 2.3 illustrates the electronic control- and signal pathways. The depletion laser pulses served as the internal trigger signal for the FPGA unit. One function of the FPGA unit was to operate the four galvanometric mirrors ( $\mathrm{X} 1, \mathrm{X} 2, \mathrm{Y} 1$ and $\mathrm{Y} 2$ ) of the Quadscanner (QS) by individually applying voltages

\footnotetext{
${ }^{6}$ Transmission $>90 \%$
} 
to define the scan position and the scan area in $x y$-direction. For precise focus adjustment, the expansion of the piezoelectric transducer (zPiezo) was varied in $z$-direction by the FPGA unit. Furthermore, the motorized pinhole (motPinhole) was set by the FPGA unit to enable variable diameter sizes. Essentially, the FPGA unit controlled the emission of excitation and depletion lasers in terms of intensity modulation, fast on/off-switching, and synchronized timing. The delay between excitation and depletion laser pulses was adjusted to optimally deplete the fluorescence signal. Beside the manipulation of the laser emission, the FPGA unit enabled software control to set the gate width and gate delay of time-gated detection, which recorded the fluorescent signal following the depletion laser pulse.

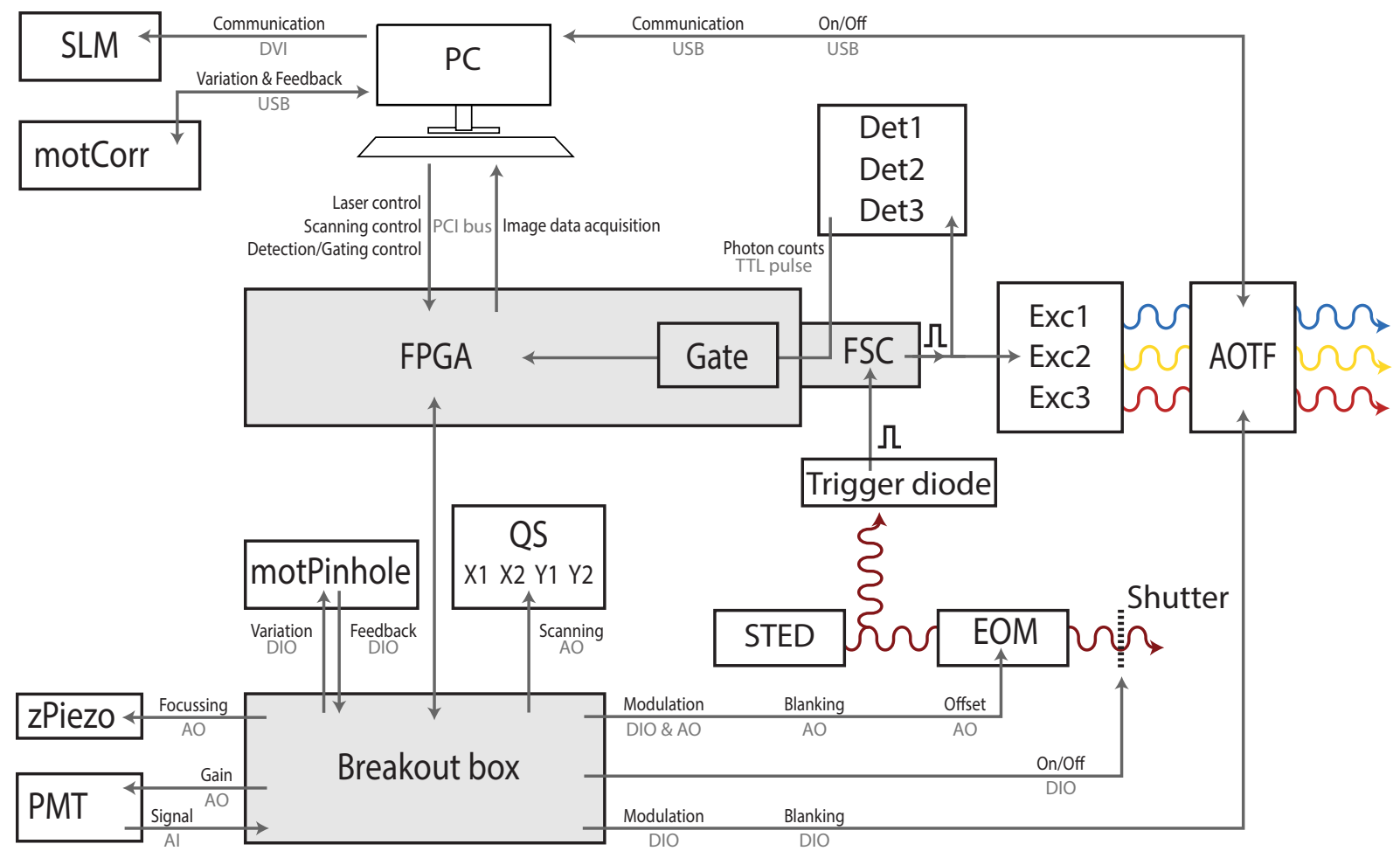

Figure 2.3: Schematic of electronic control- and signal pathways

Hardware control of the STED nanoscope was implemented by an FPGA board, an I/O breakout box, and an I/O fast signal connector (FSC). Л: trigger/delay, Al: analog input, AO: analog output, AOTF: acousto-optic tunable filter, Det: detector, DIO: digital input/output, DVI: digital visual interface, EOM: electro-optic modulator, Exc: excitation laser, motCorr: motorized aberration correction collar of objective lens, motPinhole: motorized confocal pinhole, PMT: photomultiplier tube, QS: Quadscanner, SLM: spatial light modulator, USB: universal serial bus, zPiezo: piezoelectric transducer for focus adjustment.

\section{Hardware control}

The STED nanoscope and the image acquisition were controlled by a graphical user interface ${ }^{7}$ that was programmed in LabVIEW (2014 Service Pack1, version: 14.0.1f3, National Instruments). The SLM and the motorized aberration correction collar of the objective lens (motCorr) were operated individually. The commercially available software from PiezoMotor allowed the aberration correction collar of the objective lens to be rotated precisely. An encoder integrated in the piezoelectric rotary motor transmitted the absolute position data. For the implementation of a doughnut-shaped focal intensity distribution and aberration correction of the depletion beam, an interface ${ }^{8}$ in MATLAB (R2016b, version: 9.1.0.441655, The MathWorks, Inc.) was used to flexibly control the SLM output on the liquid-

\footnotetext{
${ }^{7}$ Custom-made by Johann Engelhardt, German Cancer Research Center (DKFZ), Heidelberg, Germany

${ }^{8}$ Custom-made by Joachim Fischer, DKFZ
} 
crystal display, whose size was $792 \times 600$ pixels with a pixel pitch of $12.5 \mu \mathrm{m}$. The SLM modulates the phase of the beam by varying the applied voltages to the single pixels, which change the orientation of the birefringent parallel-aligned liquid-crystal molecules individually (software: Hamamatsu Photonics, 2014; hardware: Hamamatsu Photonics, 2014). For one linear incident polarization, different tilt angles result in different refractive indexes, which lead to a phase difference due to different optical path lengths (Hamamatsu Photonics, 2014). Using the MATLAB interface, the SLM output was controlled by sending gray-scale images representing the desired phase pattern from the PC to the controller of the SLM via the digital video interface. The SLM controller processed the image signal into voltages and displayed the phase image on the liquid-crystal of the SLM by changing the orientation of the liquid-crystal molecules accordingly (software: Hamamatsu Photonics, 2014; hardware: Hamamatsu Photonics, 2014).

(a)

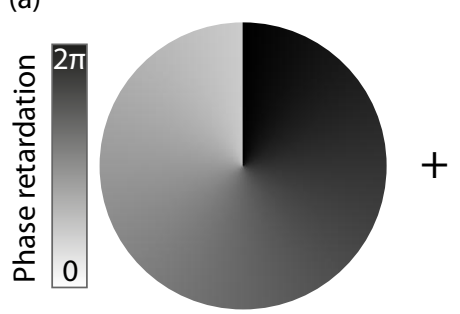

(b)

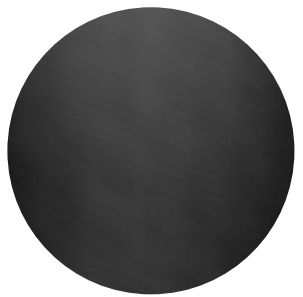

(c)

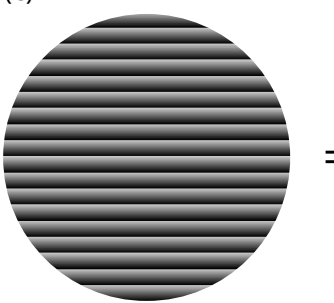

(d)

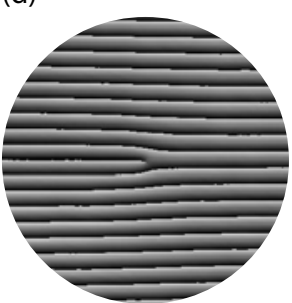

Figure 2.4: Phase pattern displayed on the SLM

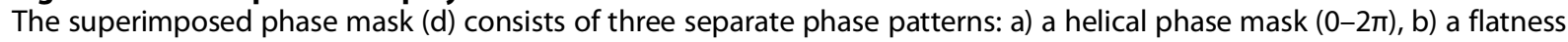
correction pattern, and c) a blazed grating. The superposed phase pattern was displayed on the SLM to generate the doughnut-shaped beam profile with a central singularity in the focal plane.

A phase image contains phase values from 0 to $2 \pi$ radians and is represented as an 8-bit gray level image. Depending on the wavelength, a $2 \pi$ phase may correspond to a gray value of 255 or less, as determined by the manufacturer-provided scaling constant. The desired final phase image comprised at least three superimposed images. i) A helical phase mask (0-2 $\pi$ ) (Figure 2.4a) was employed for the generation of the characteristic doughnut-shaped focal depletion pattern. ii) A wavelengthdependent flatness correction pattern (Figure 2.4b), provided by the manufacturer, was applied to correct the surface distortion by the liquid-crystal itself. This pattern ensured that the dedicated phase modulation was not altered by intrinsic distortions of the SLM. iii) A blazed grating (Figure 2.4c) was utilized to spatially separate phase-modulated light (first diffraction order) from undesired nonphase-modulated light (zero diffraction order). The latter was blocked by an aperture (see Figure 2.1) to prevent contribution to the image formation process. Optionally, aberration correction of the depletion beam could be performed by applying an additional aberration correction phase pattern represented in terms of Zernike polynomials ${ }^{9}$. The superimposed image (sum of at least three images) was rescaled to an 8-bit gray-scale image by phase wrapping ${ }^{10}$, if the result of superpositioning exceeded the maximum gray level value of 255 . Figure $2.4 \mathrm{~d}$ shows the final phase pattern which includes the helical phase mask, the flatness correction pattern, and the blazed grating as displayed on the SLM.

\footnotetext{
${ }^{9} \mathrm{~A}$ common way to mathematically describe the shape of an optical wavefront is with Zernike polynomials, which were formulated by Fritz Zernicke in 1934. The Zernike polynomials are a complete set of functions, which are orthogonal and continuous over the unit circle (Zernicke, 1934). These make them suitable for accurately describing optical wavefront aberrations of optical systems with circular pupils.

${ }^{10}$ Only gray level values from 0 to 255 can be displayed on the SLM. Due to superimposing of several phase pattern, some pixel values exceeded the maximum gray level value of 255 . These pixel values were set to the remainder after division by 256 (modulo operation) (software: Hamamatsu Photonics, 2014; hardware: Hamamatsu Photonics, 2014).
} 


\subsection{Animal procedures}

According to the guidelines of the European and national law, the animal care and all mouse procedures of the present study were approved by the Niedersächsisches Landesamt für Verbraucherschutz, Germany. We used two different mouse strains depending on the labeling strategy and made all efforts to reduce the number of animals for our experiments.

- C57BL/6N wildtype mice, in-house-bred

- PSD95-HaloTag

PSD-95-HaloTag knock-in mice (C57BI/6J background) expressing endogenous postsynaptic density 95 (PSD-95) protein fused with a HaloTag; the HaloTag replaced the stop codon behind the last exon (new stop codon was engineered after the HaloTag sequence), from Prof. Seth G. N. Grant, Centre for Clinical Brain Sciences, Edinburgh University, Edinburgh, United Kingdom or in-house-bred; publication on generation of PSD95-HaloTag mice is in preparation

Male mice with an age of three to six months were used in all conducted in vivo experiments. In case of PSD95-HaloTag, we used both heterozygous and homozygous mice.

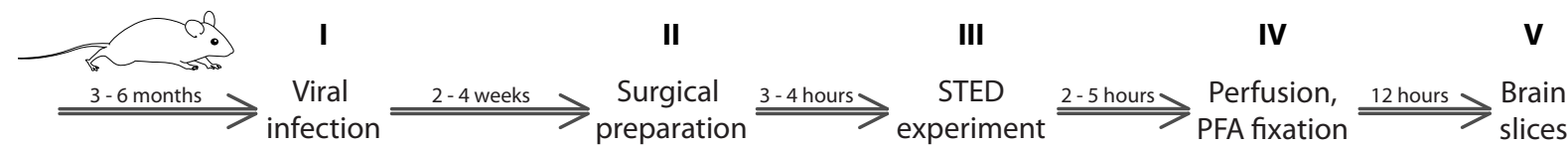

Figure 2.5: Flow chart of animal experiments

A virus was injected in the brain of adult mouse (I). The virus needed two to four weeks to reach an adequate expression level. Surgical intervention (II) was performed to enable in vivo STED imaging (III). After the imaging, mouse was transcardially perfused with paraformaldehyde (PFA) (IV). The brain was collected and processed to prepare brain slices for histology (V).

For the in vivo application of the STED nanoscope, several different procedures were conducted with the mouse as chronologically illustrated in Figure 2.5. We started with a viral infection (see Section 2.2.1) to achieve fluorescent labeling of the structure of interest. Depending on the expression level, we performed in vivo STED imaging two to four weeks after viral infection. The STED imaging came along with prior surgical preparations (see Section 2.2.2) comprised of a central venous catheterization for infusion anesthesia, a tracheotomy for artificial respiratory ventilation, the stereotactic fixation of the skull, and the preparation of a cranial window. For a histological control afterwards, we executed mouse fixation via transcardial perfusion with paraformaldehyde (PFA) (see Section 2.2.4) after finishing in vivo imaging. Finally, we prepared coronal brain slices to identify the locations of the labeled structures.

In the following sections, the surgical preparation for in vivo imaging is described in detail. We performed mouse surgery similar as previously reported (Cetin et al., 2006; Holtmaat et al., 2009; Berning et al., 2012; Willig et al., 2014) and we optimized some surgical steps specifically needed for nanoscale STED imaging to achieve a reliable preparation with minimal movement artifacts. Throughout the viral infection, the surgical preparation, and the entire in vivo STED imaging, we continuously monitored and documented the depth of anesthesia and vital parameters of the mouse. Heart rate, breath rate, and arterial oxygen saturation were recorded by a pulse oximeter (MouseOx Plus, Starr Life Sciences Corp., Oakmont, Pennsylvania, USA). Mouse was positioned on a heating plate (home-built) to maintain the body temperature $\left(35-37^{\circ} \mathrm{C}\right)$ and a rectal thermal probe (GTF 300 and GTH-1170, GHM Messtechnik, Regenstauf, Germany) was used for body core temperature controlling. 


\subsubsection{Viral infection}

In order to label the structure of interest with a fluorescent protein or with a HaloTag in the visual cortex, we performed stereotactic intracranial injection of adeno-associated viruses in mouse two to four weeks before STED imaging.

Table 2.1 lists all recombinant adeno-associated virus (rAAV) vectors, which were used to target different structures of interest. Some of the viral plasmids were designed in-house (see Appendix A.6 for plasmid sequences) and were already present in the department. Virus particles were obtained by transfecting HEK 293FT cells with (i) the PAAV plasmid encoding the desired fusion construct, (ii) pF $\Delta 6$ containing adenoviral helper proteins for replication, (iii) pH 21 and pRV1 encoding replication and capsid proteins of serotype 1 and 2, respectively. Two days after virus transduction, cells were resuspended in lysis buffer $(150 \mathrm{mM} \mathrm{NaCl}, 50 \mathrm{mM}$ Tris- $\mathrm{HCl}, \mathrm{pH} 8.5)$ and cracked by three freeze-thaw cycles $\left(-80^{\circ} \mathrm{C}\right.$ for $20 \mathrm{~min}, 37^{\circ} \mathrm{C}$ for $\left.10 \mathrm{~min}\right)$. After a $5 \mu \mathrm{l} \mathrm{DNAseI} \mathrm{treatment}\left(1 \mathrm{U} / \mu \mathrm{l} ; 37^{\circ} \mathrm{C}\right.$ for $\left.30 \mathrm{~min}\right)$ the lysate was centrifuged ( $1690 \mathrm{~g}$ for $10 \mathrm{~min}$ ) to remove the cell fragments. Afterwards, the supernatant was centrifuged at $4^{\circ} \mathrm{C}$ (48000 $\mathrm{g}$ for two hours) and the pellet containing the virus particles was resuspended in $150 \mu \mathrm{l}$ sterile filtered phosphate-buffered saline (PBS, pH 7.5). $5 \mu \mathrm{l}$ aliquots were frozen and stored at $-80^{\circ} \mathrm{C}$.

\begin{tabular}{ll} 
Table 2.1: Adeno-associated viral vectors \\
\hline F-actin labeling & rAAV1/2-hSyn-Actin-Chromobody-mNeptune2 ${ }^{11}$ \\
& rAAV1/2-hSyn-Lifeact-mNeptune2.5 \\
& rAAV1/2-hSyn-Lifeact-tagRFP65 $7^{11}$ \\
& rAAV1/2-hSyn-Lifeact-mCardinal ${ }^{12}$ \\
& rAAV1/2-hSyn-Lifeact-mGarnet ${ }^{11}$ \\
& rAAV1/2-hSyn-Lifeact-YFP12 \\
& rAAV1/2-hSyn-Lifeact-Halo' \\
& rAAV1/2-hSyn-eGFP ${ }^{11}$ \\
\hline Cytosol labeling & F-actin: filamentous actin; hSyn: human synapsin promoter
\end{tabular}

For the viral infection, all surgical steps were performed under sterile conditions to reduce the risk of inflammations. Surgical equipment was cleaned with $70 \%$ ethanol and all substances were applied using a syringe filter $(0.45 \mu \mathrm{m}$ pore size, Whatman, Maidstone, UK). For preemptive analgesia, carprofen $(5 \mathrm{mg}$ per $\mathrm{kg}$ of body weight; $50 \mathrm{mg} / \mathrm{ml}$ carprofen in sterile physiological $0.9 \% \mathrm{NaCl}$ solution; Rimadyl, Pfizer, New York City, New York, USA; NaCl, B. Braun Melsungen, Melsungen, Germany) was injected subcutaneously. After $\geq 30 \mathrm{~min}$, the mouse was anesthetized by an intraperitoneal injection of pentobarbital sodium (77 mg per $\mathrm{kg}$ of body weight; $16 \mathrm{~g} / 100 \mathrm{ml}$ pentobarbital sodium dissolved in sterile physiological $0.9 \% \mathrm{NaCl}$ solution; Narcoren, Merial, Hallbergmoos, Germany; NaCl, B. Braun Melsungen). The anesthetized mouse was placed in proneposition on a heating plate (home-built). The head was stabilized in a stereotactic frame (SG-4N, Narishige, Tokyo, Japan). Eyes were protected from dehydration by applying dexpanthenol (eye and nose ointment, Bepanthen, Bayer Vital, Leverkusen, Germany), and breathing gas (mixture of $47.5 \%$ oxygen, 50\% nitrogen, and $2.5 \%$ carbon dioxide; Westfalen AG, Münster, Germany) was administered by a respiratory mask (home-built). The head was shaved (Moser ChroMini Pro, Wahl, Unterkirnach, Germany), the scalp was disinfected with $70 \%$ ethanol, and surgically opened by a $5 \mathrm{~mm}$ incision

\footnotetext{
${ }^{11}$ Design, production, and/or purification of viral vector were performed by C. Gregor, P. llgen, T. Gilat, or J. Hubrich, all Max Planck Institute for Biophysical Chemistry, Göttingen, Germany.

${ }^{12}$ Plasmid and/or viral vector were kindly provided by the laboratory of Dr. K. I. Willig of University of Göttingen, Germany.
} 
above the left parietal bone. A hole with a diameter of $0.7 \mathrm{~mm}$ was drilled (drill bit: HD 1RF007, Hager \& Meisinger, Neuss, Germany; drill: Ideal Micro-Drill, CellPoint Scientific, Gaithersburg, Maryland, USA) through the skull of the left parietal bone at $4 \mathrm{~mm}$ caudal distance from bregma ${ }^{13}$ and $2.5 \mathrm{~mm}$ medial-to-lateral distance from the midline suture to access the area of the visual cortex. To avoid injuries of the cortical surface, drilling was only performed until blood vessels in the dura were clearly visible through the thin, but still intact skull. The drilling hole was filled and covered with sterile artificial cerebrospinal fluid (ACSF) at pH 7.4 containing $126 \mathrm{mM} \mathrm{NaCl}, 2.5 \mathrm{mM} \mathrm{KCl}, 2.5 \mathrm{mM} \mathrm{CaCl} 2$ $2 \mathrm{H}_{2} \mathrm{O}, 1.3 \mathrm{mM} \mathrm{MgCl}{ }_{2} 6 \mathrm{H}_{2} \mathrm{O}, 27 \mathrm{mM}$ HEPES, and $30 \mathrm{mM}$ glucose, and the trepanation was perforated carefully with a forceps to expose the cortex surface.

(a)

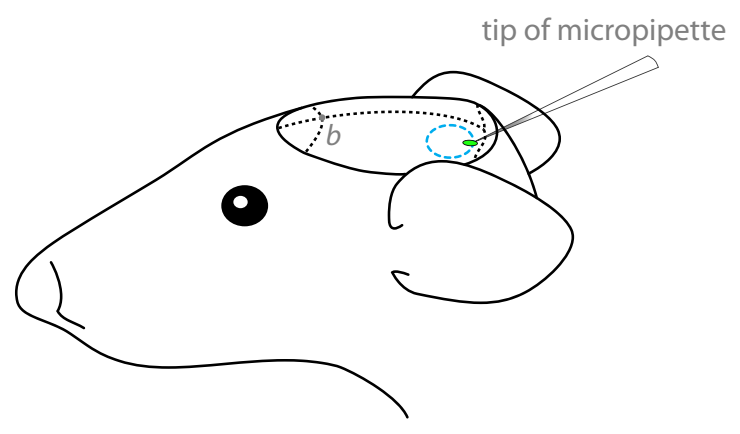

(b)

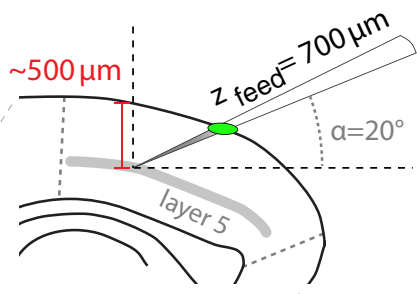

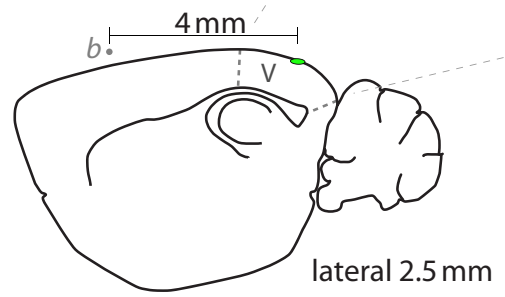

Figure 2.6: Schematic of viral injection into the visual cortex of the left hemisphere

(a) The virus was injected with a fine pre-pulled glass micropipette through a drilled hole (green spot) in the left parietal bone located at the caudal edge of the supposed imaging area (blue circle). Dotted lines mark the three prominent bone sutures used as the primary guides. (b) Sagittal brain section (modified from Paxinos and Franklin, 2007) with the position of the trepanation ( $4 \mathrm{~mm}$ caudal from bregma $b$ and $2.5 \mathrm{~mm}$ lateral from the midline suture) and with the stereotactic coordinates (feed $z_{\text {feed, }}$ angle $a$ ) to reach layer 5 of the visual cortex (V) at depths of around $500 \mu \mathrm{m}$ from the cortical surface.

The trepanation was placed $\sim 0.5-1 \mathrm{~mm}$ caudal to the supposed imaging area, since the virus was stereotactically injected by a pre-pulled glass micropipette (tip diameter $\sim 10 \mu \mathrm{m}$ (1B150F-4 with inner diameter $\mathrm{ID}=0.84 \mathrm{~mm}$ and outer diameter $\mathrm{OD}=1.5 \mathrm{~mm}$; World Precision Instruments, Sarasota, Florida, USA)) under an angle of $20^{\circ}$ using a micromanipulator (SM-11, Narishige) (Figure 2.6a). The capillary micropipette was inserted with a feed of $\sim 700 \mu \mathrm{m}$ from the brain surface to reach the cell bodies of the pyramidal neurons of layer 5 of the visual cortex located at a depth of $\sim 500 \mu \mathrm{m}$ from the cortical surface (Figure 2.6b). The caudal trepanation location and the $20^{\circ}$ insertion of the micropipette minimized brain tissue injuries especially in the imaging region. The virus ( 250 to $500 \mathrm{nl}$ (1:5 diluted in sterile ACSF)) was gently injected using air pressure pulses applied via a pressure generator ( 30 to 50 msec pulses with 0.7 to 3.5 bar; both parameters were adjusted to modulate the flow rate; TooheySpritzer, Toohey Company, Fairfield, New Jersey, USA). The micropipette retained in its position for three minutes to allow virus diffusion into the tissue and to prevent subsequent backflow of the virus into the injection site. After retraction of the micropipette, the scalp incision was closed with three single-button sutures and the mouse was placed back in its cage (supported on a heating plate) to recover. The mouse fully recovered within one to three hours after surgery and returned to the animal facility under normal housing conditions until the STED imaging experiment. For post-operative care, the mouse was analgetically aided for three days with metamizole ( $375 \mathrm{mg}$

\footnotetext{
${ }^{13}$ The bregma was used as a landmark for stereotactic targeting. It is formed by the intersection of the coronal and sagittal sutures of the skull (Cetin et al., 2006).
} 
per $250 \mathrm{ml}$ in the drinking water; $500 \mathrm{mg} / \mathrm{ml}$ metamizole dissolved in water; Novaminsulfonratiopharm, ratiopharm, Ulm, Germany).

\subsubsection{Surgical preparation of the mouse for STED imaging}

Two to four weeks after viral infection, the mouse was pre-operatively analgized with carprofen ( $5 \mathrm{mg}$ per $\mathrm{kg}$ of body weight; $50 \mathrm{mg} / \mathrm{ml}$ caprofen in sterile physiological $0.9 \% \mathrm{NaCl}$ solution; Rimadyl, Pfizer; $\mathrm{NaCl}, \mathrm{B}$. Braun Melsungen) and initially anesthetized using pentobarbital sodium (77 mg per $\mathrm{kg}$ of body weight; $16 \mathrm{~g} / 100 \mathrm{ml}$ pentobarbital sodium dissolved in sterile physiological $0.9 \% \mathrm{NaCl}$ solution; Narcoren, Merial; $\mathrm{NaCl}$, B. Braun Melsungen) for the STED imaging experiment. The depth of anesthesia was verified by checking sensorimotor reflexes e.g. pinch of hindpaw. Dexpanthenol was applied to both eyes to prevent them from dehydration during surgery and STED imaging. The mouse was placed in supine position on a heating plate (home-built) and upper and lower limbs were fixed in place with surgical tape (Leukosilk, BSN medical, Hamburg, Germany). The head was straightened by tethering a yarn around the front teeth, and breathing gas (mixture of $47.5 \%$ oxygen, $50 \%$ nitrogen, and $2.5 \%$ carbon dioxide; Westfalen AG) was administered by a respiratory mask (home-built).

In the first step, the throat was shaved and exposed by a $20 \mathrm{~mm}$ long median skin incision between the sternum and the larynx. The lobes of the salivary glands and connective tissue were separated gently with forceps and arranged sideways to gain access to the right external jugular vein. For vascular catheterization, two yarn loops were positioned with $5 \mathrm{~mm}$ distance along the exposed jugular vein. The distal loop was tightly closed to interrupt the blood flow, and a small incision was made in between. A tube catheter (Portex ${ }^{\mathrm{TM}}$ Fine-Bore Polyethylene Tubing with $I D=0.28 \mathrm{~mm}$ and $\mathrm{OD}=0.61 \mathrm{~mm}$, Smiths Medical, St. Paul, Minnesota, USA) was inserted into the incision of the jugular vein to allow a continuous anesthesia infusion of methohexital sodium $(50 \mathrm{mg}$ per hour and $\mathrm{kg}$ of body weight; $500 \mathrm{mg}$ methohexital sodium dissolved in sterile physiological $0.9 \% \mathrm{NaCl}$ solution; Brevimytal, Hikma, London, UK; $\mathrm{NaCl}$, B. Braun Melsungen) by using a syringe pump (BS-300, Braintree Scientific, Braintree, Massachusetts, USA). The tube catheter was fixed by the two loops, and additionally to the skin at the frontal part of the median skin incision. During the entire experiment, the heart rate was kept between 270 to 375 beats per minute by varying the dose of anesthesia. To reduce motion artifacts in the images, which might result from active respiration during imaging, artificial ventilation of the mouse through intubation of the trachea was necessary. To expose the trachea, the sternohyoid muscles were spread bluntly along the midline. Two yarn loops were positioned around the trachea with $7 \mathrm{~mm}$ spacing. Here, an incision was made between two tracheal cartilages, located close to the thymus. A home-built tube was inserted into the trachea and secured in place by using the two yarn loops. The tracheal tube was designed in a T-shape. One distal end ( $1.35 \mathrm{~mm} \mathrm{OD}$ ) was used for insertion into the trachea. The other two parts served as the flow-in of the breathing gas mixture $(1.1 \mathrm{~mm} \mathrm{OD})$ as well as the flow-out of the out-breathing (2.1 mm OD). Pancuronium bromide (100 $\mu \mathrm{l}$ per hour from $0.095 \mathrm{mg} \mathrm{ml}^{-1}$ stock solution; $4 \mathrm{mg} / 2 \mathrm{ml}$ pancuronium bromide dissolved in sterile physiological $0.9 \% \mathrm{NaCl}$ solution; Pancuronium Inresa, Inresa Arzneimittel, Freiburg, Germany; $\mathrm{NaCl}$, B. Braun Melsungen) was injected subcutaneously for muscle relaxation. Thereafter, the artificial ventilation (home-built) was kept at 120 breaths per minute with a tidal volume of $120-140 \mu \mathrm{l}$. This flat respiration pattern was used to minimize the mouse movement during imaging. The breathing gas mixture exhibited a high percentage of carbon dioxide which is needed to avoid respiratory alkalosis. Danger of malfunction of the lungs by alveolar 
collapse was prevented by a short deep breath when necessary (arterial oxygen saturation below $92 \%$, controlled by pulse oximetry).

After closing the throat incision with suture clips the mouse was placed in prone position. Two stainless steel needle electrodes $(0.33 \mathrm{~mm}$ diameter) were subcutaneously inserted into the forepaws for electrocardiogram recordings monitored using a differential preamplifier (home-built) in combination with an oscilloscope. The head of the mouse was shaved and a flap of the scalp, covering the skull of both hemispheres, was surgically removed. Then, the periosteum was carefully scraped off the bone with a curette and Histoacryl tissue adhesive (B. Braun Melsungen, Melsungen, Germany) was applied to the wound margins to keep the skull dry (adopted from Holtmaat et al., 2009). A flat, tiltable head holder (home-built) was adhered with dental cement (Super-Bond CB, Sun Medical Co., Moriyama City, Japan) on the frontal bone of the skull between the eyes (see Figure 2.7). Insufficient removal of the periosteum or inadequate drying of the skull would prevent a proper bonding of dental cement and result in mechanical instability. The holder served specifically to mechanical stabilize the mouse head for further surgical preparation steps as well as for the subsequent imaging experiment. While previous work (Holtmaat et al., 2009; Berning et al., 2012; Willig et al., 2014) used a simple bar shaped head holder, we designed a T-shaped head holder to provide a larger surface contact area to the bone of the skull for a more reliable stabilized head fixation. In addition, we tested different dental cements and achieved the best bonding with SuperBond $\mathrm{CB}^{14}$. Other dental cements, like Fuji Ortho LC (GC Corp., Tokyo, Japan), had less bonding efficiency and lost their bonding to the skull within hours. Both improvements extended the mechanical stability and reduced the motion artifacts due to sufficient and stable bonding between the animal and the head holder, and resulted in a robust connection to the $x y z$-translation stage of the STED nanoscope.

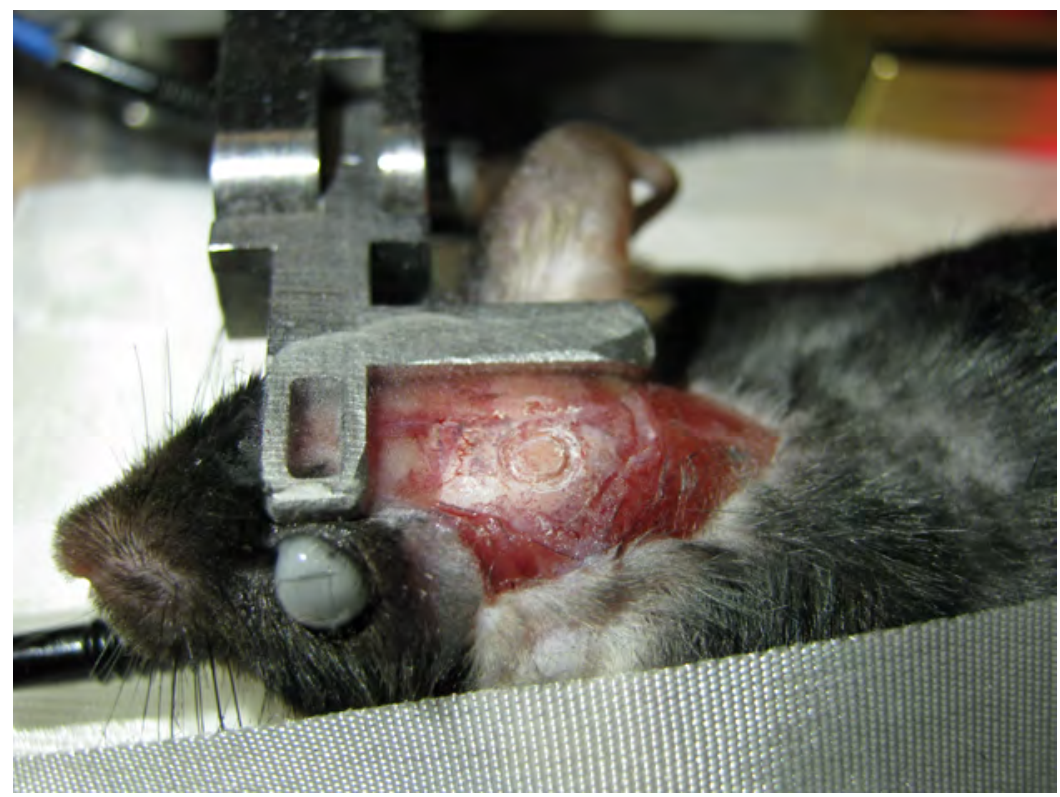

Figure 2.7: Mouse head fixation

A tiltable head holder (home-built) was adhered with dental cement to the skull of an anaesthetized mouse to mechanical stabilize the animal for the preparation of the cranial window and more important for the subsequent STED imaging experiment.

\footnotetext{
${ }^{14}$ Upon suggestion of J. Tereshchenko from the Neurobiological Research Laboratory, University Hospital Göttingen, Germany
} 
After the dental cement had hardened, a circular groove ( 2 to $3 \mathrm{~mm} I D$ ) was drilled into the skull around the region of interest (Figure 2.8a, see also Figure 2.6 and Figure 2.7), comprised the former trepanation made for the viral infection. In addition, a straight groove was drilled to enable access for a fine tube (150 $\mu \mathrm{m}$ OD) filled with ACSF and connected to a $1 \mathrm{ml}$ syringe. This fine tube served to extract spare cerebrospinal fluid during the imaging experiment if required (Figure 2.8b). A large drop of ACSF was applied to the circular cranial bone, before it was lifted and removed using sharp forceps. Residual bone material and arachnoid mater were eliminated with fine tipped forceps (Figure 2.8c), leading to a convex curvature of the brain. The opened brain area was washed with fresh ACSF.
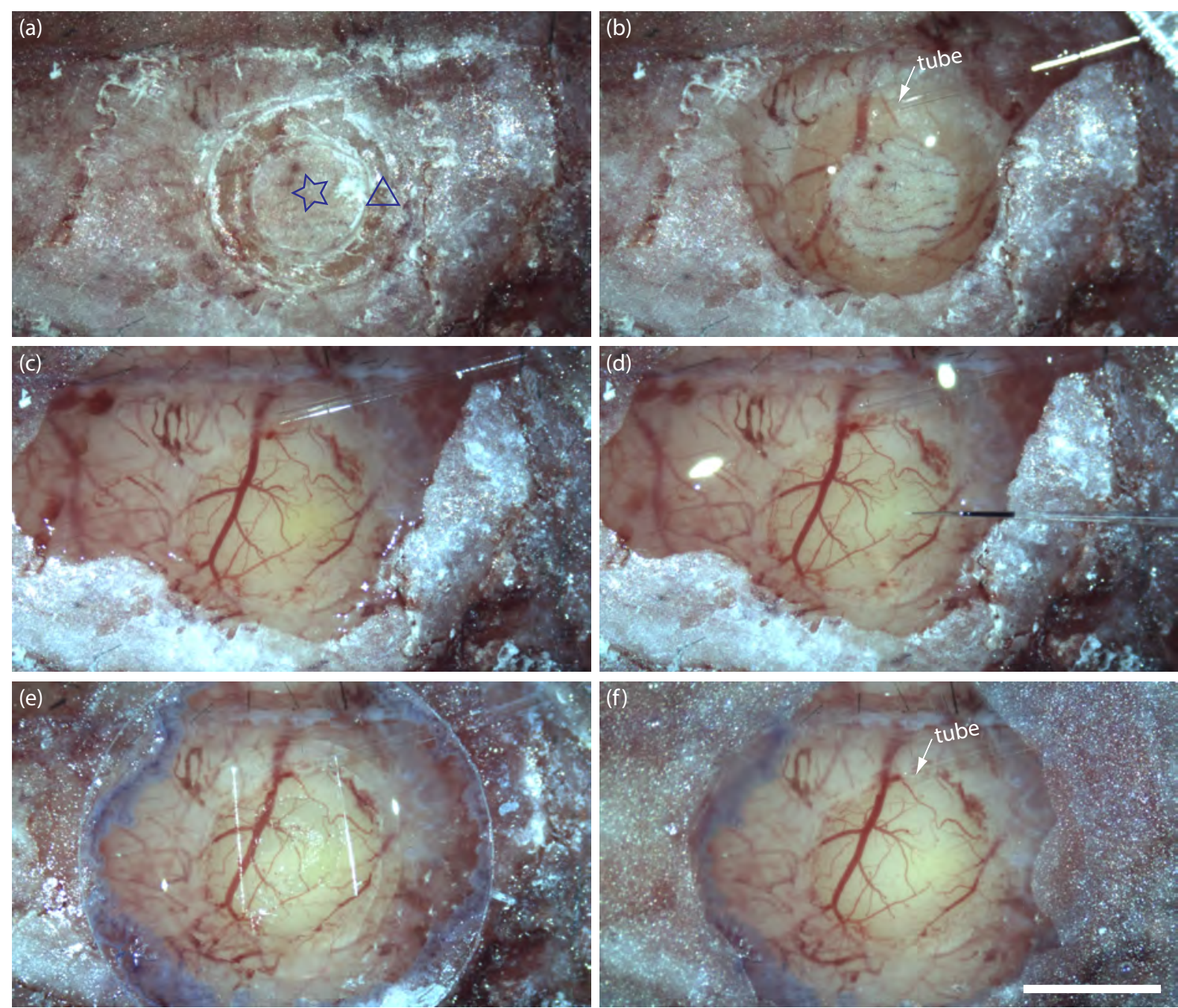

Figure 2.8: Surgical preparation of the cranial window

(a) A circular groove of 2 to $3 \mathrm{~mm}$ ID was drilled into the skull around the region of interest (asterisk, supposed viral infection area), comprised the former trepanation of the viral infection (triangle). (b) A drainage tube was implemented into the groove to be able to extract spare cerebrospinal fluid, and the region of interest was covered with sterile ACSF. (c) After removal of circular cranial bone and arachnoid mater, the cortex with its superficial blood vessels became clearly visible. (d) If applicable, fluorescent organic dye was stereotactic injected by using a pre-pulled glass micropipette. (e) A circular cover glass, covered with a thin layer of a protective polymer and a piece of transparent adhesive tape, was glued to the skull with Histoacryl tissue adhesive (slightly visible in blue). (f) Finally, the exposed skull, the edges of the cover glass, and the head holder were covered with dental cement. Scale bar: $2 \mathrm{~mm}$. 
Depending on the fluorescent labeling approach, a stereotactic injection of fluorescent organic dye was carried out on several locations in the center region of the cranial window (Figure 2.8d) as described in Section 2.2.3. Afterwards, the cranial hole was covered with a circular cover glass ${ }^{15}$ ( $6 \mathrm{~mm}$ diameter, \#1.5 thickness; Thermo Fisher Scientific, Waltham, Massachusetts, USA). Through the convex bow of the brain, the cortex with the pia mater formed direct contact to the glass coverslip in the center of the optical window. A small amount of Histoacryl tissue adhesive was applied around the cover glass for sealing the optical window (Figure 2.8e). Afterwards, exposed skull, wound margins, edges of the cover glass, and the head holder were covered with dental cement to form a mechanical solid unit (Figure 2.8f). Before the heating plate with the head-fixed mouse was transferred onto the $x y z$-translation stage of the STED nanoscope for imaging, we adjusted the orientation of the mouse head. With the tiltable head holder and the dumping screws of the heating plate the glass surface of the cranial window was positioned perpendicular to the optical axis of the nanoscope.

\subsubsection{Fluorescent organic dye injection}

By using genetically encoded HaloTags fused to the protein of interest, a fluorescent organic HaloTag-ligand needed to be applied for labeling the target structure. Therefore, we strove for a direct injection of the fluorescent dye into the center of the supposed imaging area and established a method for labeling in vivo. Table 2.2 lists the fluorescent organic dyes as HaloTag-ligands which were used to label the HaloTag fusion proteins. The ligands were provided as $100 \mu \mathrm{M}$ or $5 \mathrm{mM}$ stock solutions (Facility for Synthetic Chemistry, Max Planck Institute for Biophysical Chemistry, Göttingen) and were diluted with ACSF to lower concentrations for experimental use.

Table 2.2: Fluorescent organic dyes

\begin{tabular}{|c|c|c|c|}
\hline Dye & Stock solution & Dilution & Manufacturer \\
\hline 580CP-Halo ${ }^{[1]}$ & $5 \mathrm{mM}$ in DMSO & $750 \mathrm{nM}$ in ACSF & in-house \\
\hline 610CP-Halo ${ }^{[1]}$ & $100 \mu \mathrm{M}$ in DMSO & $750 \mathrm{nM}$ in ACSF & in-house \\
\hline 630GeRH-Halo ${ }^{[2]}$ & $5 \mathrm{mM}$ in DMSO & $750 \mathrm{nM}$ in ACSF & in-house \\
\hline GeR-Halo ${ }^{[2]}$ & $5 \mathrm{mM}$ in DMSO & $750 \mathrm{nM}$ in ACSF & in-house \\
\hline SiR-Halo ${ }^{[3]}$ & $100 \mu \mathrm{M}$ in DMSO & $750 \mathrm{nM}$ in ACSF & $\begin{array}{l}\text { in-house, commercially available from } \\
\text { Spirochrome Ltd, Stein am Rhein, } \\
\text { Switzerland }\end{array}$ \\
\hline $640 \mathrm{SiRH}-\mathrm{Halo}^{[2]}$ & $100 \mu \mathrm{M}$ in DMSO & $750 \mathrm{nM}$ in ACSF & in-house \\
\hline
\end{tabular}

DMSO: dimethyl sulfoxide; ACSF: artificial cerebrospinal fluid; CP: carbopyronine; GeR: germanorhodamine; h: hydroxylated; SiR: silicon-rhodamine; [1]: Butkevich et al., 2016; [2]: Butkevich et al., 2017; [3]: Lukinavičius et al., 2013;

A pre-pulled glass micropipette with $\sim 10 \mu \mathrm{m}$ tip diameter (1B150F-4 with ID $=0.84 \mathrm{~mm}$ and $\mathrm{OD}=1.5 \mathrm{~mm}$; World Precision Instruments) was stereotactically inserted with a feed of $100 \mu \mathrm{m}$ into the visual cortex under an angle of $20^{\circ}$ to the brain surface (see Figure 2.9) by using the same micromanipulator as for viral infection (see Section 2.2.1). Between 100 to $250 \mathrm{nl}$ HaloTag-ligand ( $750 \mathrm{nM}$, dissolved in ACSF) was injected to layer 1 of the visual cortex of the left hemisphere by using

\footnotetext{
${ }^{15}$ The cortex facing surface of the cover glass was coated with a thin layer of $100 \mathrm{~nm}$ crimson fluorescent spheres as described in Section 2.4 in order to use them as a reference for measurement of the depth of the fluorescent labeling. If the distance between the fluorescent spheres and the fluorescent-labeled structures was larger than $5 \mu \mathrm{m}$, spare cerebrospinal fluid was the cause of the spacing in most of the cases and was extracted by using the provided drainage tube. The other surface of the cover glass was prepared with a layer of a protective polymer (First Contact Polymer, Photonic Cleaning Technologies, LLC, Platteville, WI, USA), covered by a piece of transparent adhesive tape. Both protect the cover glass surface from scratches or dirt during mounting to the skull.
} 
a pressure generator ( 30 to 50 msec pulses with 0.7 to 3.5 bar). The micropipette retained in its position for around $30 \mathrm{sec}$ to allow dye diffusion into the tissue. Usually, this procedure was performed on four to eight different locations within the center of the region of interest in between the superficial blood vessels.

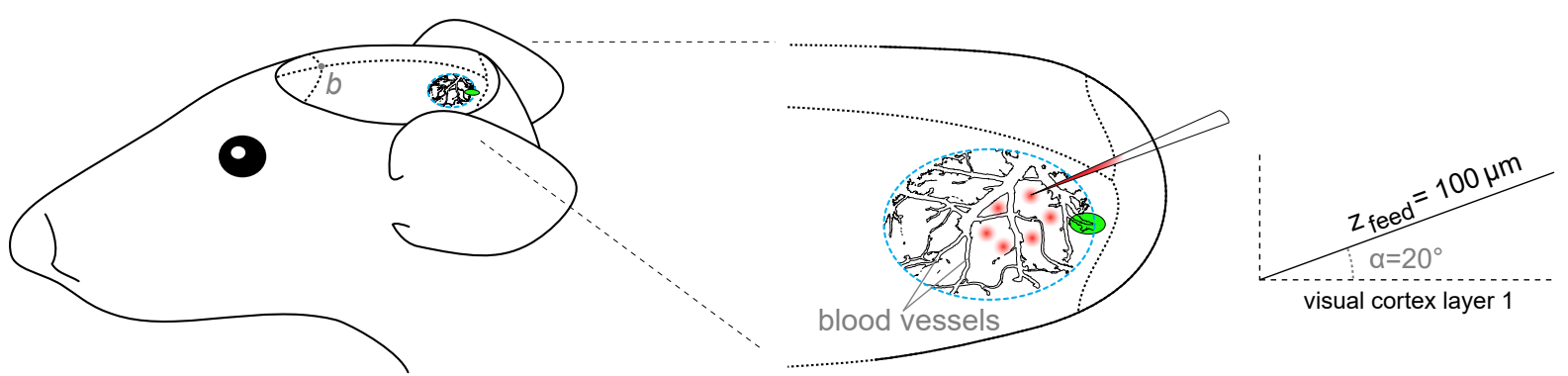

Figure 2.9: Schematic of fluorescent dye injection into the visual cortex of the left hemisphere

Fluorescent dye was injected with a micropipette at several locations (red spots) within the supposed imaging area (blue circle) of the opened skull at the left parietal bone. Stereotactic injections were performed with a feed of Zfeed $=100 \mu \mathrm{m}$ under an angle of $\mathrm{a}=20^{\circ}$ to reach layer 1 of the visual cortex.

\subsubsection{Preparation and imaging of fixed brain slices}

For histological control, the mouse was transcardially perfused with PBS (pH 7.4), followed by $4 \%$ PFA ( $\mathrm{pH} 7.4$ ). The brain was dissected and post-fixed in $4 \%$ PFA (pH 7.4) at $4^{\circ} \mathrm{C}$. On the next day, the solution was replaced with PBS, containing $0.5 \%$ PFA, for preservation during storage of the brain at $4^{\circ} \mathrm{C}$ until sectioning. Both hemispheres were separated along the mid-sagittal plane and the left hemisphere was embedded in $3 \%$ agarose in PBS (pH 7.4). Series of coronal sections of $70 \mu \mathrm{m}$ thickness were cut with a vibratome (VT1000S, Leica) and mounted on coverslips using Mowiol.

Images of fixed coronal brain slices were acquired with an epifluorescence microscope (DM6000 B, Leica) using magnifications of 2.5/5/10/20/40× (N PLAN 2.5×/0.07, HC PL FLUOTAR 5×/0.15, HC PL FLUOTAR 10×/0.30, HC PL FLUOTAR 20×/0.55, HCX PL FLUOTAR 40×/0.75, all Leica Microsystems). Blue (BP 470/40) and red (BP 600/37) light was applied to excite the fluorescence of yellow and red markers, respectively.

\subsection{Cell culture}

Vero cells were grown on coverslips and cultured at $37^{\circ} \mathrm{C}$ with $5 \% \mathrm{CO}_{2}$ and $90 \%$ humidity in Dulbecco's Modified Eagle Medium (DMEM, cat. no. 61965-059, Thermo Fisher Scientific/Gibco, Waltham, Massachusetts, USA) enriched with $10 \%$ fetal bovine serum (cat. no. S0615, Biochrom $\mathrm{GmbH}$, Berlin, Germany), 1\% sodium pyruvate (1 mM final concentration; cat. no. S8636, SigmaAldrich Corp., St. Louis, Missouri, USA), and 1\% penicillin/streptomycin (100 U/ml and $100 \mu \mathrm{g} / \mathrm{ml}$ final concentration, respectively; cat. no. A2213, Biochrom).

The culture preparation was carried out by the technician Tanja Gilat from Max Planck Institute for Biophysical Chemistry, Göttingen.

Cultures of hippocampal neurons were prepared from newborn Wistar rats (postnatal day P0-P1) of both sexes following the same procedure as described in (D'Este et al., 2015). Neuronal cultures ( 100.000 cells) were grown on $18 \mathrm{~mm}$ glass coverslips covered with $100 \mu \mathrm{g} / \mathrm{ml}$ poly-L-ornithine (cat. no. P3655, Sigma-Aldrich) and $1 \mu \mathrm{g} / \mathrm{ml}$ laminin (cat. no. 354232, BD Bioscience, San Jose, California, USA). Cells were cultured at $37^{\circ} \mathrm{C}$ with $5 \% \mathrm{CO}_{2}$ in neurobasal medium (cat. no. 21103049 , Thermo 
Fisher Scientific/Gibco) enriched with serum-free B27 (50x) (cat. no. 17504044, Thermo Fisher Scientific/Gibco), L-glutamine (1 mM final concentration; cat. no. 25030024, Thermo Fisher Scientific/Gibco), and penicillin/streptomycin $(100 \mathrm{U} / \mathrm{ml}$ and $100 \mu \mathrm{g} / \mathrm{ml}$ final concentration, respectively; cat. no. A2213, Biochrom). One day after preparation of neuronal cultures, the growth medium was supplemented with cytosine $\beta$-D-arabinofuranoside ( $5 \mu \mathrm{M}$ final concentration; cat. no. C1768, Sigma-Aldrich).

The culture preparation was carried out by the technicians Ina Herfort and Jasmine Hubrich, both Max Planck Institute for Biophysical Chemistry, Göttingen, in accordance with the regulations of the German Animal Welfare Law and under institutional guidelines. The use of organs and tissues collected from sacrificed animals is not an animal experiment as stated in the European Union Directive (2010/63/EU). Therefore, no approval for the preparation of neuronal cultures was required.

\subsection{Alignment and test samples}

Gold bead samples To align the depletion pattern and to precisely co-align the depletion and excitation beams, we used gold bead samples. Gold nanoparticles with a diameter of $80 \mathrm{~nm}$ (EM.GC80, British Biocell International, Cardiff, United Kingdom) diluted 1:100 in $\mathrm{H}_{2} \mathrm{O}$ were sonicated for $15 \mathrm{~min}$. A square coverslip (22 mm x $22 \mathrm{~mm}$ with thickness No. 1.5H, Paul Marienfeld, Lauda Königshofen, Germany) was covered with $150 \mu$ l poly-L-lysine (P8920-100ML, Sigma-Aldrich) for three minutes, washed with deionized water and dried with pressurized air. The gold bead dilution was applied to the coated coverslip, incubated for five minutes, rinsed off with deionized water, and dried with pressurized air. In a final step, the coverslip was mounted onto a microscope slide in oilimmersion ${ }^{16}$ (Type B, Cargille, Cedar Grove, New Jersey, USA) and sealed with nail polish.

\section{Fluorescent bead samples}

For regular alignment and performance tests of the custom-built STED nanoscope, we prepared calibration samples with commercially available fluorescent spheres of different diameter sizes (see Table 2.3). These fluorescent spheres are loaded with an ensemble of fluorescent dye molecules inside a polystyrene sphere that provides enough brightness and shows little to no photobleaching. Figure 2.10 presents the normalized absorption and emission spectra of the yellow-green and crimson beads.

Table 2.3: Overview of selected fluorescent spheres

\begin{tabular}{l|c|c|c|c}
\hline Name & Size & $\begin{array}{c}\text { Excitation } \\
\text { peak } \\
\mathrm{nm}\end{array}$ & $\begin{array}{c}\text { Emission } \\
\text { peak } \\
\mathrm{nm}\end{array}$ & Catalog no. \\
\hline Yellow-green & 40 & 505 & 515 & F8795 \\
\hline Crimson & 100 & 625 & 645 & 17 \\
\hline Crimson & 40 & 625 & 645 & 17 \\
\hline Crimson & $20^{18}$ & 625 & 645 & F8782 \\
\hline
\end{tabular}

\footnotetext{
${ }^{16}$ Because only gold nanoparticles attached to the coverslip were imaged, the refractive index of the immersion medium did not affect the image quality.

${ }^{17}$ This size of crimson beads is no longer available.

${ }^{18}$ Sphere diameter is determined by electron microscopy and shows a coefficient of variation of about $20 \%$ (Thermo Fisher Scientific/Invitrogen).
} 
For the sample preparation, fluorescent spheres were diluted 1:10000 in $\mathrm{H}_{2} \mathrm{O}$ and sonicated for $15 \mathrm{~min}$. A coverslip (22 mm x $22 \mathrm{~mm}$ with thickness No. 1.5H, Paul Marienfeld, Lauda Königshofen, Germany) was coated with $150 \mu \mathrm{l}$ poly-L-lysine (P8920-100ML, Sigma-Aldrich, St. Louis, Missouri, USA) for three minutes, washed with deionized water, and dried with pressurized air. Afterwards, $50 \mu \mathrm{l}$ of diluted spheres were applied to the coverslip, incubated for three minutes, and washed in the same way as before. Finally, the coverslip was mounted with Mowiol on a microscope slide. Samples were dried for one hour before sealing with nail polish.

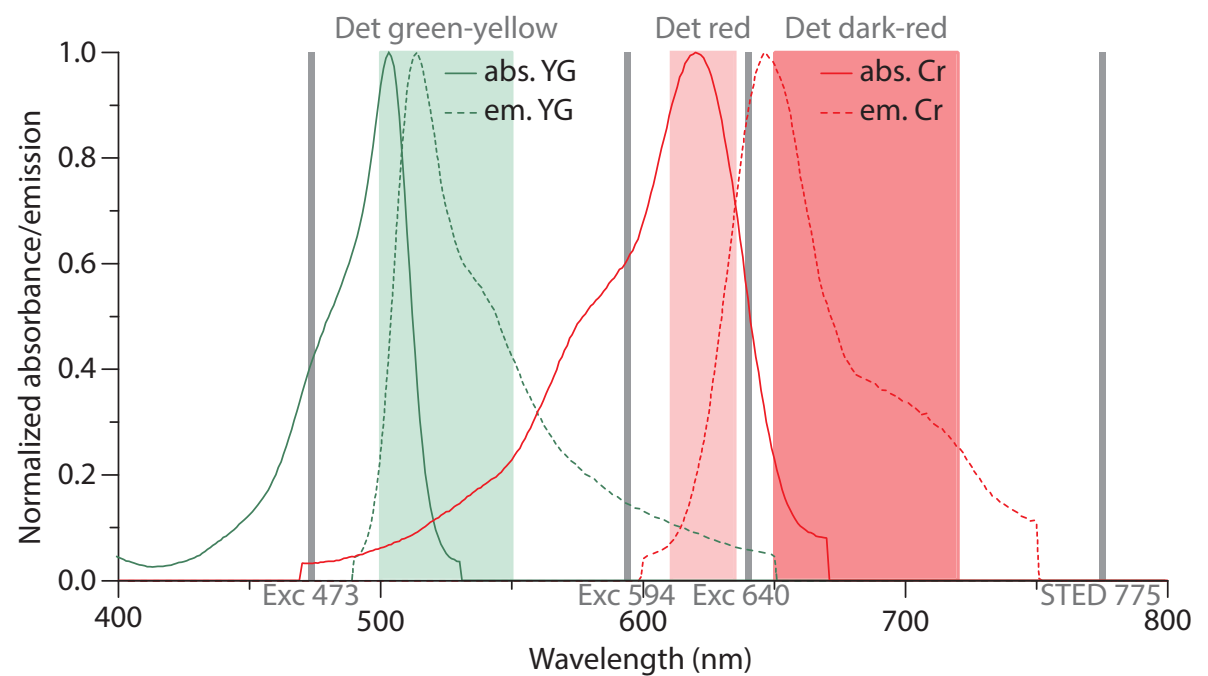

Figure 2.10: Normalized absorbance and emission spectra of selected fluorescent spheres

Absorbance (abs) and emission (em) spectra of yellow-green (YG, green curves) and crimson ( $\mathrm{Cr}$, red curves) fluorescent spheres are illustrated by solid and dashed lines, respectively. Grey bars highlight laser lines of the custom-built STED nanoscope and shaded boxes represent their designated detection channels.

\subsection{Image acquisition}

Image acquisition was performed with a custom-written LabVIEW interface ${ }^{19}$ and allowed a flexible adjustment of imaging parameters such as pixel size in $x y z$-scanning directions, field-of-view, pixel dwell time, excitation wavelength and power, depletion power, delay between excitation and depletion laser pulses, detection channel, gated detection (delay and width), imaging mode (lineby-line ${ }^{20}$ or frame-by-frame). Table A.1 lists in detail the used acquisition parameters for all fluorescent images within this thesis. Laser powers were measured in the pupil of the objective lens. The maximum field-of-view was $130 \mu \mathrm{m} \times 130 \mu \mathrm{m}$ accomplished by the Quadscanner in combination with the $63 \times$ magnification objective lens. To reduce the bleaching of the sample, all used lasers were blanked during the non-imaging return of the galvanometer mirrors of the beam scanner. In most of the experiments, STED and confocal images were acquired line-by-line to minimize drift errors between both images. If not indicated otherwise, all images are presented in raw data.

For in vivo STED imaging, the far-red fluorescent markers were excited with $594 \mathrm{~nm}$ or $640 \mathrm{~nm}$, were depleted at $775 \mathrm{~nm}$, and imaged using the custom-built STED nanoscope. The morphological reference structures labeled with either enhanced green fluorescent protein (eGFP) or yellow

\footnotetext{
${ }^{19}$ Custom-made by Johann Engelhardt, German Cancer Research Center (DKFZ), Heidelberg, Germany

${ }^{20}$ Quasi-simultaneous acquisition of confocal and STED images was enabled by recording one line of the image in the STED mode followed by an acquisition in the confocal mode of the same line.
} 
fluorescent protein (YFP) were excited at 473nm. Throughout all in vivo experiments, we acquired images at a depth of 5 to $25 \mu \mathrm{m}$ below the cortex surface, and adjusted the laser powers and correction collar of the objective lens individually. Typical imaging settings were composed of $15 \mu \mathrm{m} \times 15 \mu \mathrm{m}$ field-of-view, a pixel size of $20 \mathrm{~nm}$, and a pixel dwell time of $30 \mu \mathrm{s}$, resulting in a total acquisition time of $\sim 18 \mathrm{~s}$ per frame. Average excitation laser power was about 5-15 $\mu \mathrm{W}$. The amount of applied depletion laser power was dependent on the fluorophore (e.g. $66 \mathrm{~mW}$ for the SiR-Haloligand). To avoid motion artifacts induced by pulsating blood vessels of living mouse, imaging fields of view were selected in regions without prominent vasculation. To record the three-dimensional organization of the structure of interest, stacks of images (image stack or $z$-stack) 300 or $400 \mathrm{~nm}$ apart in axial direction were acquired.

\subsection{Image processing and analysis}

Image data was visualized, processed, and analyzed using the following software packages: ImSpector (version: 0.14.11616; Schönle, 2006), MATLAB (R2016b, version: 9.1.0.441655; The MathWorks, Inc., Natick, Massachusetts, USA), and OriginPro 2016 (version: b9.3.2.303; OriginLab Corp., Northampton, Massachusetts, USA). Typical processing and analyzing methods are described below, indicating the applied software. If image processing steps are not stated in the figure caption, images are in raw data.

\section{Smoothing}

To reduce noise, smoothing of the images was performed using a two-dimensional low-pass Gaussian filter with a width of one pixel, implemented in the software ImSpector.

\section{Brightness and contrast enhancement}

To enhance the brightness and/or contrast of images for better visibility, lower and upper count values of the colormap were adjusted individually, performed in the software ImSpector. Respective count values are stated in colorbars in or next to the images, if they were relevant.

\section{Overlay}

To merge two images into one single image, the images were overlaid by using the software ImSpector. Both images were color-coded with different colormaps (e.g. green and magenta) to identify the correlation as an additive composition of both (e.g. white as additive sum of green and magenta) in the superimposed image. A necessary requirement for an accurate overlay image was the absence of sample drift. If drift was apparent, we performed drift correction (see below) before an overlay was created.

\section{Drift correction}

If lateral drift between two corresponding images was present, a cross-correlation based maximum value registration algorithm was used to compensate the frame shift from image data sets. Estimated drift correction with an accuracy of one pixel was applied to both confocal and super-resolution images. The drift calculation and correction was executed with a MATLAB routine ${ }^{21}$.

\footnotetext{
${ }^{21}$ Custom-written by Jan Keller-Findeisen, Max Planck Institute for Biophysical Chemistry, Göttingen, Germany
} 


\section{Maximum intensity projection}

To generate a single two-dimensional presentation out of an image stack, images were maximum intensity projected along the $z$-direction using the software ImSpector. The value of every pixel of the resulting image is determined by the maximum intensity from all sections along the $z$-direction.

\section{Full width at half maximum (FWHM) analysis}

All quantitative analysis was performed on the raw data.

\section{a) Single structures}

For the determination of the image resolution based on a circular structure (e.g. fluorescent bead), an intensity profile (typically averaged over several pixels perpendicular to the direction of the profile) through the center of the structure was generated using the software ImSpector. Using Origin, a one-dimensional (1D) Gaussian peak function was fitted to the averaged intensity profile and the image resolution was determined by FWHM of the Gaussian curve fit.

For the determination of the image resolution based on the width of a feature (e.g. thickness of elongated structures), an intensity profile (typically averaged over several pixels perpendicular to the direction of the profile) through the desired width of the structure was generated using the software ImSpector. Using Origin, a 1D Gaussian or Lorentzian peak function was fitted to the averaged intensity profile and the image resolution was determined by FWHM of the Gaussian/ Lorentzian curve fit.

b) Confocal point spread function (PSF) estimation from any fluorescent beads

To determine the lateral and axial confocal image resolution, image stacks of single beads were recorded. Using a MATLAB routine ${ }^{21}$, the positions of single beads were identified by finding local intensity maxima of the smoothed image stack (convolution with a three-dimensional (3D) Gaussian peak function of four pixel FWHM). To identify the average PSF from all single bead images, the raw data was de-convolved with a stack containing non-zero pixels only at the bead positions by using the Richardson-Lucy algorithm (Richardson, 1972) running for 50 iterations. The result was a noisefree estimation of the confocal 3D PSF. Using the software ImSpector, intensity profiles (typically averaged over several pixels) along $x$ and $z$ through the center of the estimated 3D PSF were generated. Using Origin, a 1D Gaussian peak function was fitted to the averaged intensity profiles. The lateral and axial resolutions were determined by the FWHMs of the Gaussian curve fits. 


\section{RESULTS}

The aim of this thesis was to image for the first time the distribution and substructure of a synaptic protein in vivo by STED nanoscopy with markers in the far-red spectral range. In order to achieve this aim, we constructed a compact and stable in vivo two-color STED nanoscope for the far-red spectrum, which best accommodated the specific requirements for imaging the brains of living mice. Moreover, effective labeling strategies were developed, in particular, to exploit recently developed far-red fluorescent probes for live-cell imaging.

This chapter is divided into four parts. First, specific considerations for the new design concept of the developed far-red in vivo STED nanoscope are emphasized, followed by demonstrating the performance of the setup. Next, the developed labeling strategies for in vivo STED imaging are described and evaluated. Finally, the imaging results from STED nanoscopy of synaptic proteins in living mice are presented.

\subsection{New design concept for the compact in vivo STED nanoscope}

Maximum stability was the key aspect for the new design concept of the compact in vivo STED nanoscope. Typically, experimental STED setups are spread out in space due to the large number of optical components needed. To make the alignment easier, the components are often arranged with a lot of space in between. Long optical paths, however, are more susceptible to vibrations or drifts. This is in particular the case for upright configurations, which require an additional beam path that typically leads vertically up from the optical table to the dedicated beam entrance of the microscope stand. Besides the increased susceptibility to vibrations, the construction and the alignment are more complex in comparison to inverse designs. Due to our intended application on mice, we were not able to implement an inverse configuration of a STED nanoscope. Therefore, we developed a completely new design concept for an upright in vivo STED nanoscope to make it as compact and reliable as possible in order to ensure high stability. In the following, the specific considerations for the design of the newly developed far-red in vivo STED nanoscope (depicted in Section 2.1) are emphasized.

\section{In vivo imaging requirements}

To enable imaging in living mice, it is necessary to construct a STED nanoscope in an upright configuration according to the physiological orientation of the mouse head. Beyond that, adequate space between the objective lens of the microscope and the translation stage needs to be provided for placing the mouse and required support equipment such as a heating plate. To meet these requirements and to ensure maximum stability, we used a commercial upright microscope stand, offering a highly stable basis construction for further modifications. Due to its rigid design, the optical elements (e.g. tube and objective lens) have a fixed alignment and accordingly, further optimization is neither possible nor needed. A high stability, a large field-of-view, and binocular vision are, however, considerably more beneficial for the dedicated application.

Although the exact refractive index of our intended sample (the visual cortex of living mice) is unknown, previous studies successfully used glycerol-immersion objective lenses for superresolution nanoscopy of cultured brain slices (Urban et al., 2011) or cortex of the living mice (Berning et al., 2012; Willig et al., 2014). We therefore implemented a high-NA glycerol-immersion objective 
lens with a free working distance ${ }^{22}$ of $300 \mu \mathrm{m}$, intended for samples with a refractive index of about 1.45. This objective lens is designed for coverslips of 140 to $190 \mu \mathrm{m}$ thickness and with a correction collar for spherical aberrations. The latter can be adjusted to correct for refractive index variations occurring in the immersion medium or sample, and changes of cover glass thickness or temperature. Such spherical aberrations do appear if not compensated for sufficiently and will cause loss in resolution and signal intensity due to the elongation of the illumination focus (Urban et al., 2011). For a precise adjustment of the aberration correction collar with low vibrations while imaging, we mounted a small custom-built piezo-motorized unit to the objective lens for tuning the position of the correction collar, which could be controlled remotely.

To assure highest stability in $x-, y$ - and $z$-directions, we separately assembled a heavy and robust motorized translation stage (custom-built by Luigs \& Neumann) around the microscope stand (see Figure A.1). The translation stage provided enough space for placing the mouse and required mouse support equipment, and was designed for the increased size and weight of the specimen. By decoupling the translation stage from the microscope stand, we avoided any negative impacts between these components.

\section{Minimization}

Common ways to reduce the sensitivity to vibrations and drifts are to miniaturize the optical setup and decouple its components. We constructed the STED nanoscope in a modular fashion (depletion laser, multiple excitation lasers, STED unit, microscope stand, and detection) to ensure robustness and flexibility. By using fiber-based transmission of the optical beams between the modules (except for the microscope stand), we decoupled and therefore reduced the sensitivity to drift and at the same time simplified the alignment. The laser sources and the STED unit were therefore decoupled mechanically and thermally to avoid the mutual negative impacts.

Some of the most critical parts of every STED nanoscope are the formation of the helical phase pattern of the depletion beam, the combination of the excitation and the depletion beams, the generation of the circular polarization, and the split-up of the fluorescence from the incoming laser light to be spectrally detected. In our design, this part (dubbed as STED unit) was minimized to a size of $25 \mathrm{~cm} \times 30 \mathrm{~cm}$ by reducing the optical path lengths to their minimum and by using customdesigned elements (e.g. quad-bandpass dichroic filter from AHF analysentechnik). We implemented only the most essential optical components, additional components were outsourced into other modules. Thus, the complexity of the STED unit was reduced but however resulted in a loss of flexibility. For example, all three excitation laser beams were transmitted to the STED unit by the same optical fiber. We required only one dichroic mirror (with characteristic transmission and reflectance properties for the implemented laser lines) to overlay the depletion and excitation beams, but the fiber collimation optics did not allow to perfectly collimate all three excitation beams at once. Accordingly, the minimization of the STED unit was a compromise between the reduced optical path lengths and a less flexible and perfect adjustment of the optical components.

Due to its small size, we were able to directly mount the STED unit vertically to the camera port on top of the microscope stand without any further optics, and reduced the lengths of the optical beam paths drastically. The longest beam path length ${ }^{23}$ in the STED unit was $62 \mathrm{~cm}$ (excitation beam path length: $25 \mathrm{~cm}$, depletion beam path length: $62 \mathrm{~cm}$, detection beam path length: $38 \mathrm{~cm}$ ). Previous upright STED nanoscopes required an additional beam path to redirect the beams to the dedicated

${ }^{22}$ Comparable oil-immersion objective lenses typically offer a free working distance of only $140 \mu \mathrm{m}$ (Leica Microsystems, 2014).

${ }^{23}$ The beam path length was measured from the fiber output to the intermediate image plane, which was at the center of the scanner mirrors. 
beam entrance of the microscope stand (approximately $60 \mathrm{~cm}$ ), making the setup more susceptible to vibrations and thermal changes. With the new design of the compact STED nanoscope (modular fashion, minimized STED unit, and direct attachment to the microscope stand), we aimed for maximum stability as well as to reduce the need for frequent alignment procedures.

\section{Far-red spectral range}

STED nanoscopy has been shown to be well suited for imaging dendritic spines in cultured brain slices (Nägerl et al., 2008; Urban et al., 2011) and in the visual cortex of living mice (Berning et al., 2012; Willig et al., 2014) using the yellow fluorescent protein (YFP) and a depletion wavelength of about $590 \mathrm{~nm}$. Imaging deep in living tissue is, however, challenging, among others because of absorbance and scattering especially in the visible spectrum (Helmchen and Denk, 2005). By using red-shifted excitation and depletion light, the risk for tissue damage (phototoxicity) can be reduced due to the lower photon energy and minimal occurrence of common endogenous absorbers such as hemoglobin, melanin, cytochrome c, and water (Svoboda and Block, 1994; König, 2000; Weissleder, 2001; Wäldchen et al., 2015). In addition, imaging within the far-red spectral range benefits from reduced scattering, thus increasing the light penetration depth in tissue, and improving image contrast due to less autofluorescence. Thus, we custom-built the STED nanoscope for in vivo imaging implementing pulsed excitation ( $594 \mathrm{~nm}$ and $640 \mathrm{~nm}$ ) and pulsed depletion $(775 \mathrm{~nm})$ in the far-red spectral range, similar to the dual-color concept presented in Göttfert et al., 2013. With respect to the spectral properties of far-red fluorescent markers, we designed the setup for two different spectral detection ranges $(600-635 \mathrm{~nm}$ and $650-720 \mathrm{~nm})$ to allow for flexibility in the choice of the fluorophores and to enable two-color STED imaging. Using a single-doughnut approach for both detection channels ensured a perfect chromatic alignment of the two color channels because the central singularity in the depletion doughnut-shaped intensity pattern determines the position of the fluorescence signal in STED nanoscopy (Bückers et al., 2011; Tønnesen et al., 2011). Moreover, using a single optical fiber combining light from all three excitation lasers increased the optical colocalization accuracy.

\section{Flexible phase modulation and aberration correction of the depletion beam}

Conventionally, the required phase distribution of the depletion beam has been implemented mostly by optical vortex phase plates (e.g. RPC Photonics, Inc., Rochester, New York, USA), consisting of a glass substrate coated with a thin structured polymer layer. These optical elements can generate a stable and nearly perfect helical phase shift from 0 to $2 \pi$ radians, resulting in a doughnut-shaped focal intensity pattern with a central singularity when used with a circularly polarized beam (Kotlyar et al., 2005; Keller et al., 2007). However, vortex phase plates are very inflexible as they are made for a fixed design wavelength. The implementation of a spatial light modulator (SLM) is a complex, but more versatile approach to modulate the phase distribution with high flexibility. For every wavelength, an optimal phase pattern can be calculated and applied (Maurer et al., 2011; Patton et al., 2015). With high-precision phase-modulation characteristics, SLMs were successfully utilized in STED nanoscopes previously (Donnert et al., 2006; Auksorius et al., 2008; Gould et al., 2012). Due to the flexibility, a SLM can additionally compensate phase distortion of the beam induced by the optical setup itself or by the refractive index variations within the sample (Kubby, 2013; Booth, 2014; Booth et al., 2015). We adapted this approach and equipped the STED nanoscope with a liquidcrystal based SLM (from Hamamatsu). It was implemented to flexibly generate an arbitrary phase retardation pattern with high spatial accuracy, to perfectly co-align the excitation and depletion beams (see Section 3.2.1), and to perform aberration corrections of the depletion beam. By using a freely programmable SLM, the STED nanoscope actually became prepared for three-dimensional 
(3D) STED imaging. In future experiments, the phase pattern for a so-called bottle-beam (Klar et al., 2000; Harke et al., 2008; Wildanger et al., 2009; Osseforth et al., 2014) could be applied to additionally suppress fluorescence above and below the focal plane at the expense of some lateral resolution.

\section{Beam-scanning}

For the purpose of imaging in living mice, stage-scanning is not feasible because the weight as well as the spatial extent of both the mouse and required support equipment like the heating plate prevent a fast and precise scanning. To meet the requirement for a compact design, we implemented a home-built beam scanner (Quadscanner, Engelhardt, 2010; Görlitz et al., 2014), consisting of four galvanometric mirrors. In contrast to the conventional beam scanning concepts containing only two mirrors, this beam scanner not only offers a compact size but also induces fewer aberrations due to the specific orientation of the four galvanometric mirrors. The position and the angle of the beam for both scanning axes is decoupled and, therefore, this scanner can be aligned rather easily without a defined distance to the objective lens (Bingen et al., 2011). The integration of the Quadscanner into the custom-built nanoscope allowed us to laterally translate the illumination beam in the $x y$-plane (maximum field-of-view of $130 \mu \mathrm{m} \times 130 \mu \mathrm{m}$ ). For axially scanning, we mounted the objective lens on a piezoelectric transducer to translate the objective lens along the optical ( $z$-)axis.

\section{Stability}

Because super-resolution imaging is substantially more susceptible to electronic noise, and thermal or mechanical instability, maximum stability was one of the main issues of the new design for the upright in vivo STED nanoscope. The result was a modular setup with a compact STED unit. Due to the small size of the STED unit $(25 \mathrm{~cm} \times 30 \mathrm{~cm})$, we directly mounted it on top of an upright microscope stand (see Figure A.1). We stabilized the STED unit against vibrations with respect to the microscope stand by implementing an additional mechanical construction. We mounted a frame to the STED unit and the anti-vibration table (see Figure A.2) consisting of two vertical rails connected by a horizontal rail (both S 65-4, OWIS, Staufen i. Br., Germany). Moreover, we stabilized the vertical frame from behind by mounting an additional rail (XT95, Thorlabs) at an angle of about $50^{\circ}$ to the surface of the anti-vibration table. The stiffness in torsion and bending of the rails allowed us to build a robust mechanical support for the STED unit. In addition, we assembled a protective cover around the mechanical construction to block undesired light, acoustic noise, and air flow from the environment.

The hereafter shown performance confirmed sufficient stability of the STED nanoscope, since no vibrations were present in the point spread function (PSF) measurements and high resolution was achieved. Furthermore, the observed performance of the STED nanoscope was stable over weeks, reducing the need for frequent alignment procedures. Accordingly, we have been able to ensure thermal and mechanical stability of the compact STED nanoscope by minimizing the size of STED unit and providing adequate mechanical support. 


\subsection{Performance of the compact STED nanoscope}

In the previous section, we emphasized the specific considerations for the new design of the custombuilt far-red dual-color STED nanoscope, constructed for the purpose of super-resolving synaptic structures in living, anesthetized mice with far-red fluorescent markers. In the following, we demonstrate the performance of the STED nanoscope. In order to fulfill the preconditions for maximum STED resolution, we optimized the doughnut-shaped depletion beam pattern in the focal plane by adjusting the phase retardation with the SLM and by aligning for ideal circular polarization. Moreover, we optimized the spatial and temporal overlay of excitation and depletion beams, both of which were essential to achieve the best possible resolution. By performing the PSF measurements, the shapes of the PSFs of the excitation and depletion beams were determined. The adjustment of the temporal arrangement of excitation, depletion, and detection (timing scheme) is also described, before the confocal and super-resolution capabilities of the STED nanoscope are characterized and demonstrated.

\subsubsection{Characterization of the PSF}

Several factors influence the imaging capability of STED nanoscopy. Besides the quality of the optical components accountable for the extent of the aberrations, the intensity distribution of the excitation and depletion beams as well as their spatial and temporal overlap predominantly determine the super-resolution outcome. The most important property is, however, the contrast between the high intensity illumination at the crest of the doughnut-shaped distribution of the depletion beam and its minimal, ideally zero, intensity at the center. Residual depletion intensity in the zero of the doughnut leads to undesired suppression of fluorescence from the center and degrades the superresolution (Harke et al., 2008; Booth et al., 2015).

For achieving the best STED performance, the mechanical alignment needs to be impeccable for an optimal shape of the PSFs and ideal co-alignment of the excitation and depletion beams in $x y^{-}, x z^{-}$ and $y z$-directions is required. To measure and optimize the PSFs, we used a calibration sample with $80 \mathrm{~nm}$ gold beads and detected the reflected light of individual beads on the photomultiplier tube. Figure 3.1 compares the PSFs of the depletion beam and the $640 \mathrm{~nm}$ excitation beam, and their corresponding overlay. The intensity distribution of the depletion beam in $x y$-direction was symmetric and showed a close to zero intensity centered in the doughnut-shaped profile. The STED PSF views in $x z$ - and $y z$-direction exhibited an almost symmetric intensity distribution with straight, not tilted intensity maxima at the same position along the optical axis. During alignment, a slight system-induced astigmatism of the depletion beam was compensated by the SLM. No other optical aberrations were visible and needed to be corrected for. The PSF of the $640 \mathrm{~nm}$ excitation beam was symmetric and round in $x y$-direction, and revealed straight, marginally curved intensity maxima in $x z$ - and $y z$-directions. Both beams showed negligible optical aberrations and were well aligned for STED nanoscopy. Moreover, the overlay of the depletion beam and $640 \mathrm{~nm}$ excitation beam demonstrated an excellent overlap in all three directions. The distance between the maximum of the excitation intensity distribution and the minimum intensity of the depletion intensity profile in the focal plane was determined as $20 \mathrm{~nm}$. The maximum intensity of the excitation PSF had the same $z$ position as the intensity maxima of the depletion PSF. Note that the shape of the PSFs and the overlap were stable over weeks and mostly no further optimization was required. 

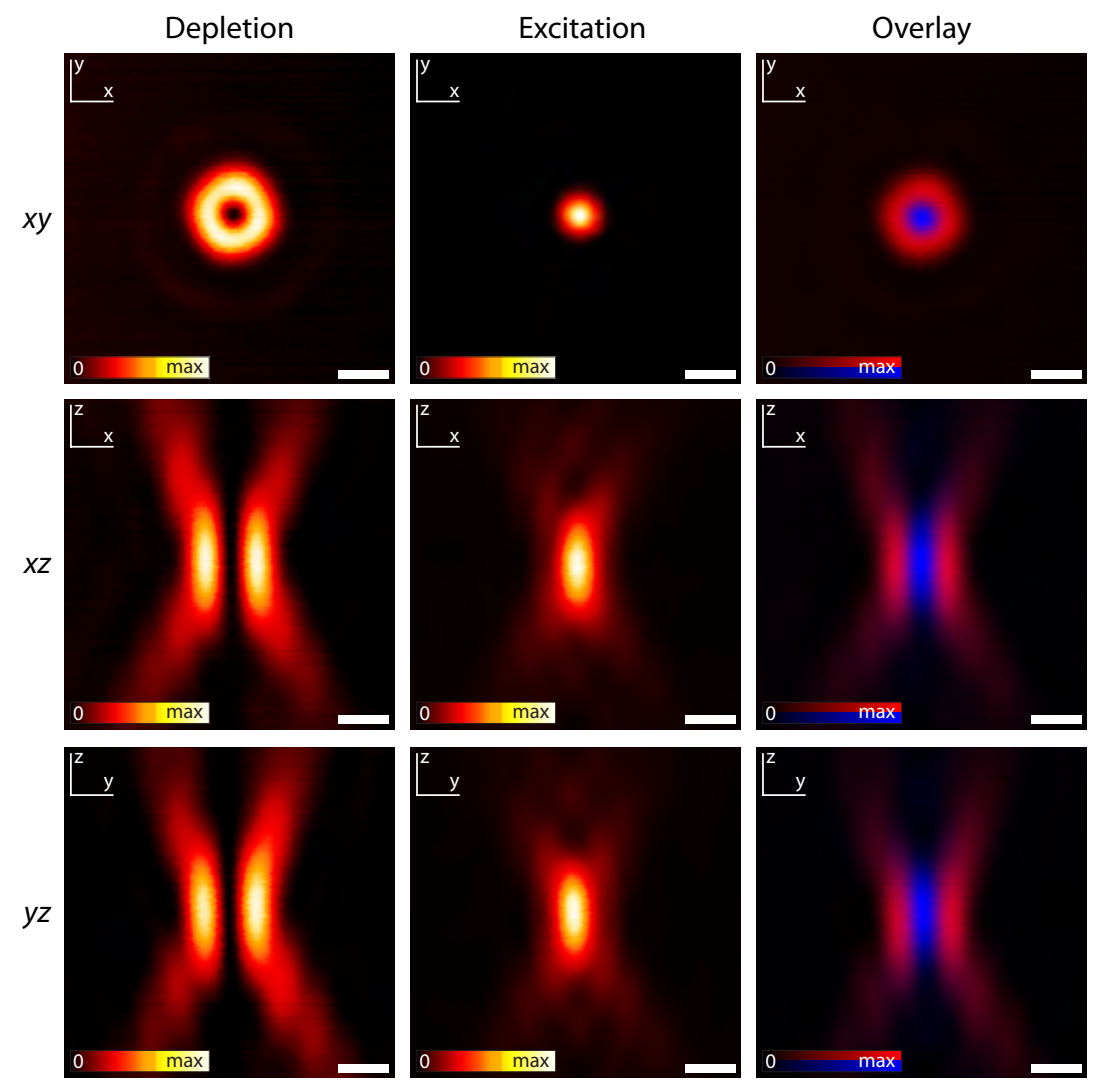

Figure 3.1: PSF measurements on $80 \mathrm{~nm}$ gold beads

The PSF of the depletion beam (left column), the PSF of the excitation beam of $640 \mathrm{~nm}$ (middle column), and the corresponding overlay (right column) of the depletion beam (red) and excitation beam (blue) are shown for $x y$ (top row), $x z^{-}$(middle row) and $y z^{-}$(bottom row) views. Scale bars: $500 \mathrm{~nm}$.

\subsubsection{Timing scheme}

Besides the spatial overlap of the depletion and excitation laser beams, the temporal alignment of both laser pulses needs to be precisely optimized when pulsed laser sources are implemented. The depletion laser pulse should be timed almost immediately after the excitation laser pulse in order to be most efficient. If the depletion pulse is initiated too early, most of the fluorescent molecules would not be in the excited state yet resulting in an overall lower STED efficiency. If the depletion pulse arrives too late, undesired spontaneous emission would occur, degrading the image contrast (Donnert et al., 2006; Vicidomini et al., 2013). Since very early fluorescence photons are likely to stem from the full diffraction-limited spot when the depletion laser pulse is not yet entirely applied, the fluorescence signal should be recorded only once the depletion pulse decayed fully. Otherwise numerous excited fluorophores at the periphery of the diffraction-limited spot would not be depleted and would lead to undesired background signal (Schrader et al., 1995; Westphal and Hell, 2005). By introducing a time-gated detection, filtering the recorded fluorescent photon based on their arrival times, the image contrast is improved due to the suppression of undesired background signal. Although the impact of time-gated detection is more pronounced for STED with continuouswave depletion lasers, an increased signal-to-background ratio has also been reported for pulsed STED nanoscopes (Moffitt et al., 2011; Vicidomini et al., 2013).

To achieve the best possible STED performance, we optimized the timing between the depletion and excitation laser pulses by using a variable electronic delay, integrated in the FPGA unit (see 
Figure 2.3). Prior coarse adjustment of the laser pulses was controlled by changing either the optical beam path length or the length of the connecting cables. By imaging $40 \mathrm{~nm}$ crimson fluorescent bead samples, we adjusted the delay of the excitation laser pulses using the depletion laser pulses as the reference. Continuous image acquisition was performed without time-gated detection and the delay of the excitation laser was varied to find the delay leading to the maximum STED resolution. We found that the excitation laser pulse should arrive about $150 \mathrm{ps}$ before the depletion laser pulse. Even though the time delay was stable over weeks, we verified the calibration by performing measurements on crimson fluorescent bead samples before every in vivo experiment. Furthermore, we were able to flexibly adjust the delay during the experiment in order to match the specific fluorophore characteristics.

To perform time-gated detection, we aligned the time gate within the FPGA unit consisting of a gate delay and a gate width for the red and dark-red detection channels. Fluorescence photons were recorded for a certain time (gate width) starting after a time delay (gate delay) with respect to the incoming depletion laser pulse (center of the laser pulse as a reference point). We experimentally investigated the resolution and contrast improvement of time-gated detection by using again $40 \mathrm{~nm}$ crimson fluorescent bead samples. The images were acquired with the $640 \mathrm{~nm}$ excitation laser and the far-red fluorescence detection channel. Figure 3.2 illustrates how the image quality was enhanced by time-gated STED in comparison to confocal and non-gated STED.
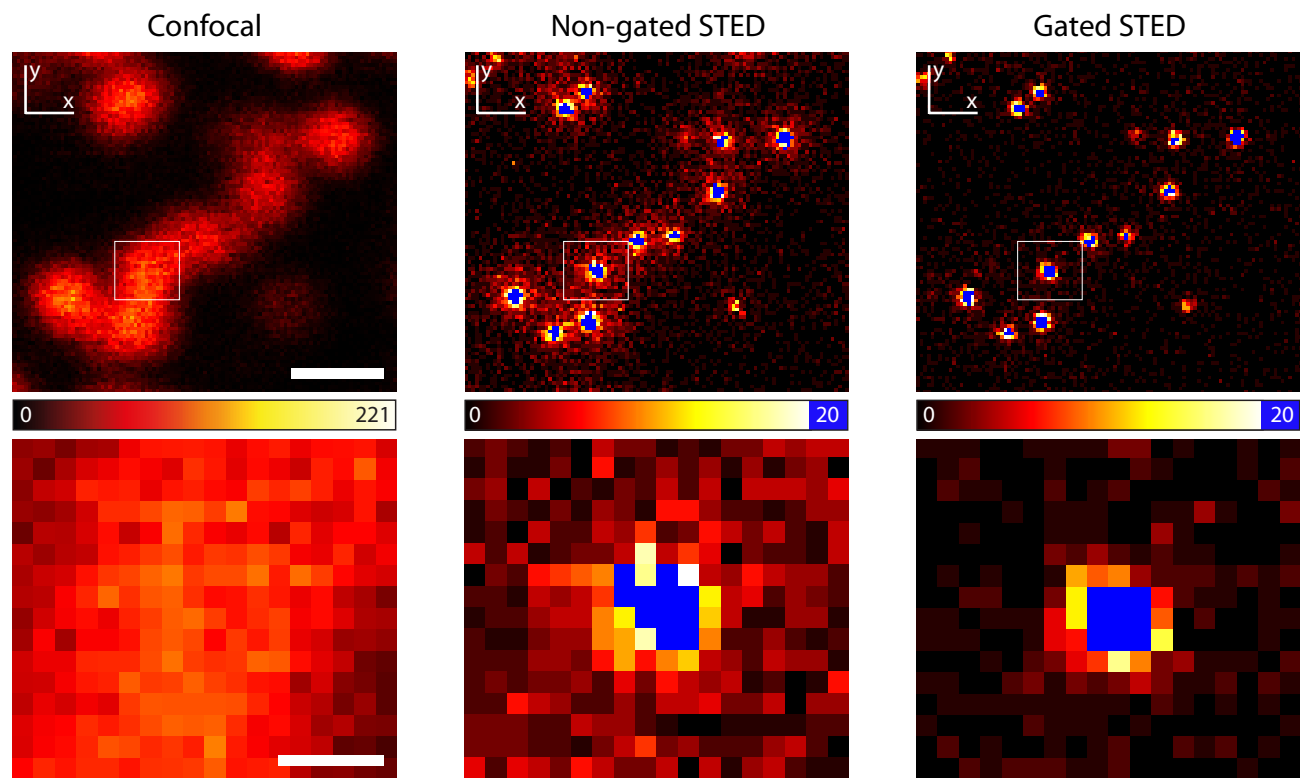

Figure 3.2: Comparison of the image quality of $\mathbf{4 0} \mathbf{~ n m}$ crimson beads by using different imaging modalities The upper row shows overview images of the same region of interest for the different imaging modes: confocal (left column), non-gated STED (middle column), and gated STED (right column). The insets are depicted in the lower row. To emphasize low counts, the color scale of the non-gated and gated STED images was cut-off at 20 counts per pixel. Scale bars: $500 \mathrm{~nm}$ (upper row), $100 \mathrm{~nm}$ (lower row).

As expected, a significant resolution enhancement was observed between the confocal and the nongated STED images. However, the non-gated STED images revealed a slight halo around the resolved beads. Primarily, this halo was caused by spontaneous emission from unquenched dye molecules, which were not depleted yet. Therefore, we implemented a gate delay to discard very early fluorescence photons. We optimized the gate delay to find the best compromise between enhanced 
image contrast and fluorescence signal loss. The maximum available gate width ${ }^{24}$ was chosen which was more than sufficient to record also the tail of the fluorescence decay. We found the best image contrast for a gate delay of $0.5 \mathrm{~ns}$ after the center of the depletion laser pulse and for a gate width of 9 ns (valid for both detection channels). In the gated STED images, the fluorescent beads were resolved without a diffraction-limited background.

To quantify the benefit of using time-gated detection for the custom-built pulsed nanoscope, we analyzed the effective PSFs of the gated and non-gated modes (see also Section A.2). Due to timegated detection, we increased the signal-to-background ratio by eliminating the diffraction-limited background almost completely, which represented $44 \%$ of the total non-gated fluorescence signal. In contrast, the total diffraction-unlimited signal was decreased only by $2 \%$. The spatial resolution, determined by measuring the average full width at half maximum (FWHM) of several beads, was identical for both detection modes. Accordingly, the implementation of time-gated detection in the custom-built pulsed STED nanoscope was beneficial due to significantly enhanced signal-tobackground ratio.

For contrast enhancement in pulsed STED nanoscopy due to time-gated detection, the gate delay should theoretically be smaller or equal to the depletion laser pulse width (Vicidomini et al., 2013). The width of the depletion laser pulses in the custom-built nanoscope was about 300 ps. However, we set a slightly larger gate delay due to the limited precision of the timing. The pulses of the depletion laser exhibited a small jitter and were stretched by an optical fiber resulting in a pulse-topulse jitter of $<20$ ps. In addition, the implemented avalanche photodiodes (APDs) have a timing resolution of about $350 \mathrm{ps}$ and the electronic processing within the FPGA unit has a jitter of $<100 \mathrm{ps}$. By using a gate delay of 500 ps, an enhanced signal-to-background ratio was achieved with negligible signal loss. The calibration of the time gate settings was performed frequently, in particular, after modifications of the setup. Typically, an adjustment of the time gate settings was not required for individual experiments.

\subsubsection{Effective confocal and STED resolution}

The characterization of the aberration-compensated doughnut-shaped intensity distribution of the depletion beam and its perfect spatial and temporal overlay with the excitation laser beam have been described in previous sections. This section outlines the confocal and super-resolution capabilities of the STED nanoscope.

Spatial resolution is a key feature of any optical microscope and is defined as the ability to distinguish between closely spaced objects (see Section 1.1). For conventional microscopes, the numerical aperture of the objective lens and the excitation wavelength are the important factors influencing the resolution of optical systems (see Equations 1.1 and 1.2). A direct measurement of the optical resolution is challenging and requires a sparse distribution of bright point-like sources of light. Fluorescent beads are the standard tool to determine the resolution of an optical system by measuring the FWHM of the PSF. The beads are advantageous due to their exceptional brightness and photostability, and are available for several wavelength ranges. The custom-built STED nanoscope had three excitation lasers $(473 / 594 / 640 \mathrm{~nm})$ and offered three detection channels (green-yellow: $497-553^{25} \mathrm{~nm}$, red: $611-635^{25} \mathrm{~nm}$, dark-red: $653-717^{25} \mathrm{~nm}$ ), with the green-yellow channel being used only in the confocal resolution. The other two detection channels were utilized

\footnotetext{
${ }^{24}$ The available time for the complete timing sequence including excitation and detection delay is limited by the repetition rate of the laser light (here: 12.5 ns pulse-to-pulse time).

${ }^{25}$ Transmission $>90 \%$
} 
for nanoscopic imaging and offered super-resolution, which is dependent on the depletion beam pattern, the depletion wavelength, the applied depletion laser intensity, and the saturation intensity of the fluorescent marker (see Section 1.3).

\section{Confocal resolution}

To estimate the confocal resolution in axial and lateral directions for the three detection channels at a detection pinhole size of 1.2 Airy units of the fluorescence wavelength of $685 \mathrm{~nm}$, we used fluorescent bead samples with yellow-green as well as crimson beads both with a diameter of about $40 \mathrm{~nm}$. Excited by the $473 \mathrm{~nm}$ laser, the yellow-green beads emitted fluorescence within the range of 503-545 $\mathrm{nm}^{26}$, which was detected by using the green-yellow channel. The crimson beads were excited by $594 \mathrm{~nm}$ and $640 \mathrm{~nm}$ separately, and the fluorescence, ranging from $630-670 \mathrm{~nm}^{26}$, was recorded with the designated detector (red or dark-red channel for $594 \mathrm{~nm}$ or $640 \mathrm{~nm}$ excitation, respectively).
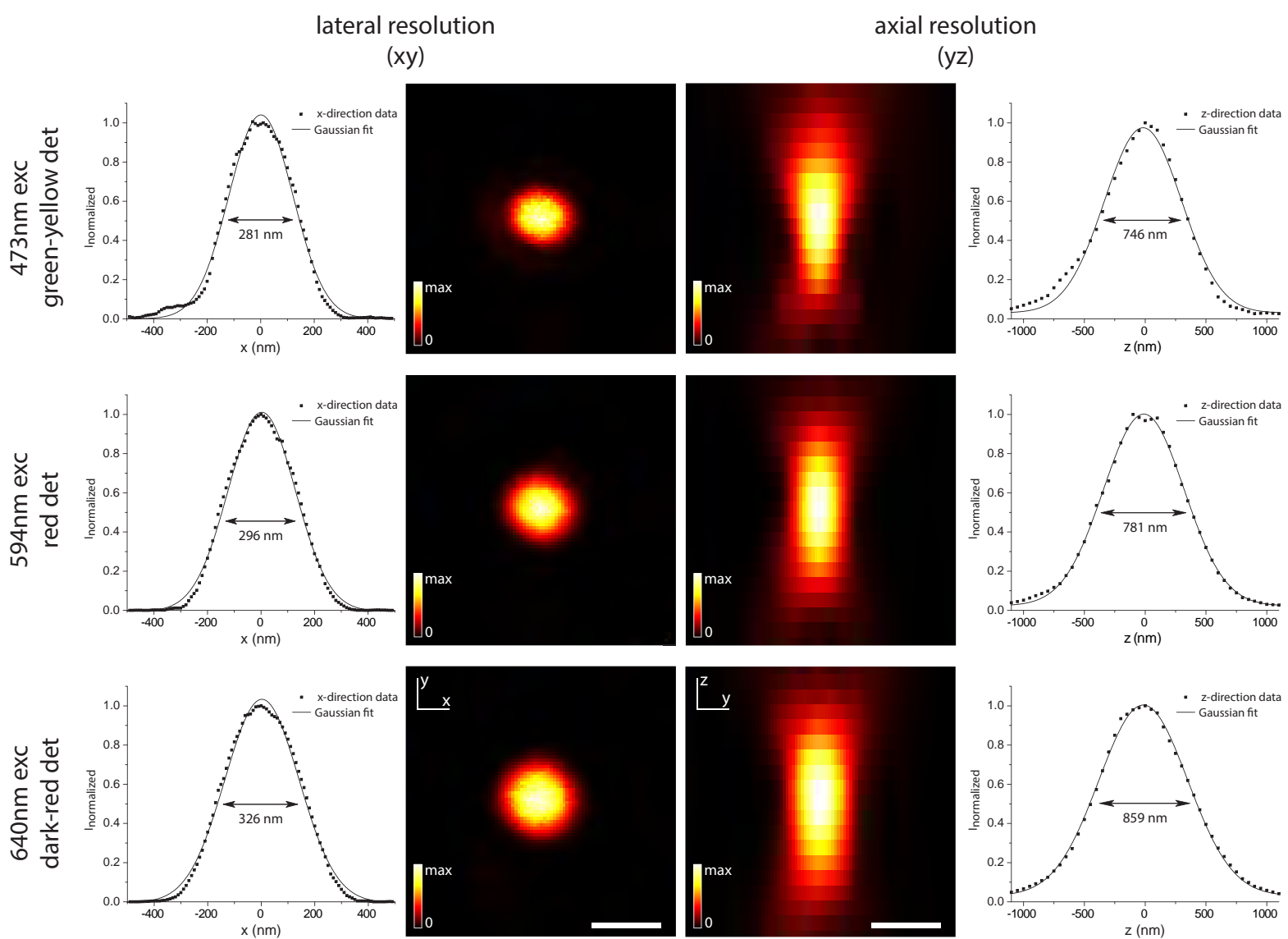

Figure 3.3: Confocal PSF estimation from images of $40 \mathrm{~nm}$ fluorescent beads (detection pinhole size of 1.2 Airy units of the fluorescence wavelength of $685 \mathrm{~nm}$ )

For each excitation-detection combination (top row: $473 \mathrm{~nm}$ excitation with green-yellow detection channel, middle row: $594 \mathrm{~nm}$ excitation with red detection channel, bottom row: $640 \mathrm{~nm}$ excitation with dark-red detection channel) confocal PSFs were estimated from image stacks of 5-10 single beads (see Section 2.6). PSFs are shown in $x y$ - and $y z$-view (both middle columns, counts are normalized). Lateral (left column) and axial (right column) resolutions were determined by FWHM of a Gaussian curve fitting to the corresponding normalized intensity profiles (averaged over 3 pixels perpendicular to the direction of the profile) through the center of the estimated PSF. Detailed imaging parameters are listed in Table A.1. Scale bars: $500 \mathrm{~nm}$.

\footnotetext{
${ }^{26}$ Relative fluorescence intensity above 50\% (Thermo Fisher Scientific/Invitrogen)
} 
For every selected combination of excitation laser and detection channel, we acquired image stacks of 5-10 single beads over a $3 \mu \mathrm{m}$ axial range with a $100 \mathrm{~nm}$ axial step size (the $z$-axis corresponds to the propagation direction of light). To analyze the lateral and axial resolution by means of the recorded image stacks, we used implemented tools of the software products ImSpector and Origin to estimate the average FWHM of several beads (see Section 2.6). The $x y$ - and the $y z$-view of the estimated PSF for each excitation-detection combination is shown in Figure 3.3 (both middle columns). For each detection channel, we calculated the lateral and axial resolution by determining the FWHM from fitted Gaussian curves (left and right column of Figure 3.3), respectively. While the PSF of the $473 \mathrm{~nm}$ excitation in the $x y$-direction was slightly elliptical due to aberrations (dimension in $x$ is longer than in $y$ ), the PSFs of $594 \mathrm{~nm}$ and $640 \mathrm{~nm}$ were almost symmetric and round. A lateral resolution of $281 \mathrm{~nm}, 296 \mathrm{~nm}$, and $326 \mathrm{~nm}$ was achieved for the green-yellow, red, and dark-red detection channels, respectively. As expected, the axial resolution was considerably lower than the lateral resolution. The PSFs of all three detection channels exhibited almost symmetric distributions with straight, not tilted intensity maxima in the $z$-direction. Only the PSF of the $473 \mathrm{~nm}$ excitation revealed noticeable spherical aberration. For the green-yellow, red, and dark-red detections, axial resolutions of $746 \mathrm{~nm}, 781 \mathrm{~nm}$, and $859 \mathrm{~nm}$ were measured, respectively.

To evaluate these experimental results, we calculated the ideal values of the lateral and axial resolution for the used wavelengths and numerical aperture of the objective lens of the STED nanoscope using vector diffraction theory (Richards and Wolf, 1959) and assuming planar illumination of the objective lens (resulting images shown in Figure A.4). For the lateral resolution, we experienced a difference of $45 \%$ for the green-yellow and $20-30 \%$ for red and dark-red channels between theoretical and experimental values. For the axial resolution, we measured $62 \%$ longer FWHM values for the green-yellow channel and about $40 \%$ longer for the red and dark-red channels than the calculated values. Due to unavoidable residual aberrations in various elements in beam paths of the system and the sample itself, the experimental resolution is always worse with a typical deviation $\sim 20 \%$ compared to theoretical calculations assuming ideal optics. During the design and construction of the STED nanoscope, we considered the most important factors influencing the resolution. The differences between the theoretical and the experimental values may result from our design concept, which was optimized to provide an almost aberration-free beam path for the depletion wavelength. We aspired to implement a non-disturbed centered zero in the doughnutshaped beam profile, which determines the super-resolution capability. Moreover, we adjusted the optics not traversed by the depletion beam for the far-red spectral range and, in consequence, the system performance of the green-yellow channel was slightly compromised.

\section{STED resolution}

To estimate the lateral STED resolution and for frequently executed performance tests of the STED nanoscope, we used crimson fluorescent bead samples with a nominal sphere diameter of $20 \mathrm{~nm}$. We recorded crimson beads, emitting fluorescence within the range of 630 to $670 \mathrm{~nm}^{27}$, in both confocal and STED modes with the maximum available depletion laser power. Figure 3.4 compares the lateral resolution of confocal (a) and STED (b) images of $20 \mathrm{~nm}$ crimson beads using the STED nanoscope with highest available depletion laser power. The confocal image shows blurred fluorescent beads, partly clustered, and fails to resolve bead aggregates. In contrast, most of the fluorescent beads are revealed separately in the STED image with excellent signal-to-noise ratio, though the peak intensity was reduced by $35.5 \%$. This is typical for STED imaging and does not

\footnotetext{
${ }^{27}$ Relative fluorescence intensity above 50\% (Thermo Fisher Scientific/Invitrogen)
} 
represent photobleaching, as can be seen on subsequent confocal images (not shown) acquired after the STED image. At highest available depletion laser power, the FWHM along a lateral direction of one exemplary bead amounted to $60 \mathrm{~nm}$, an improvement by a factor of $\sim 5.5$ over the $328 \mathrm{~nm}$ FWHM of the bead in confocal resolution.
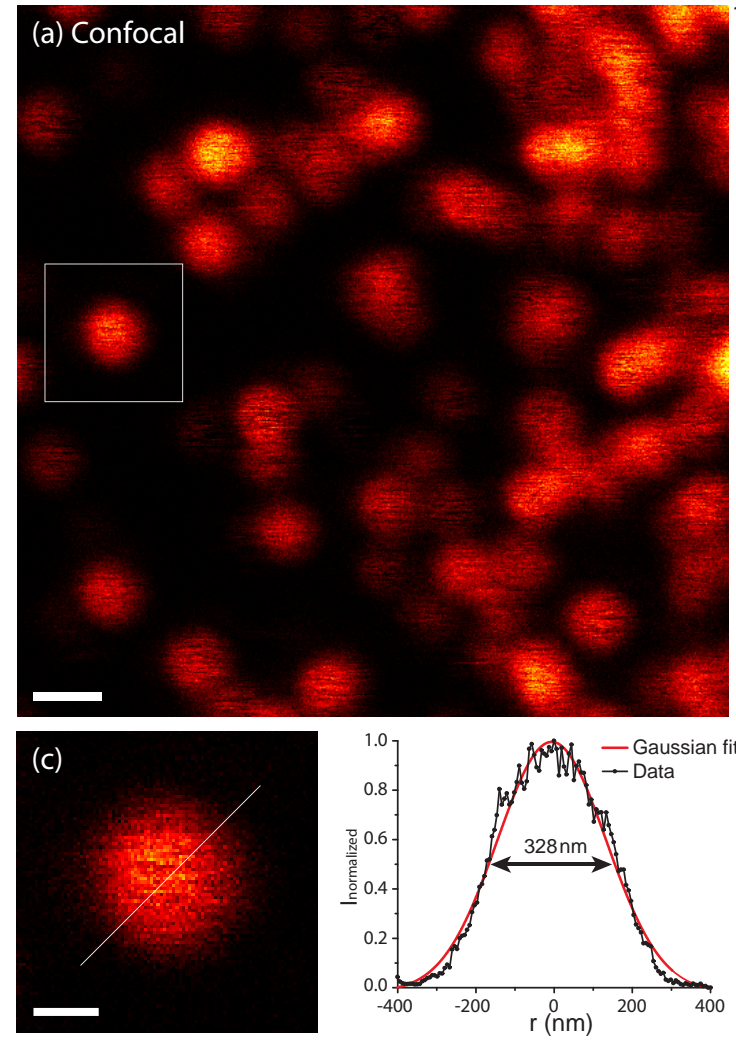
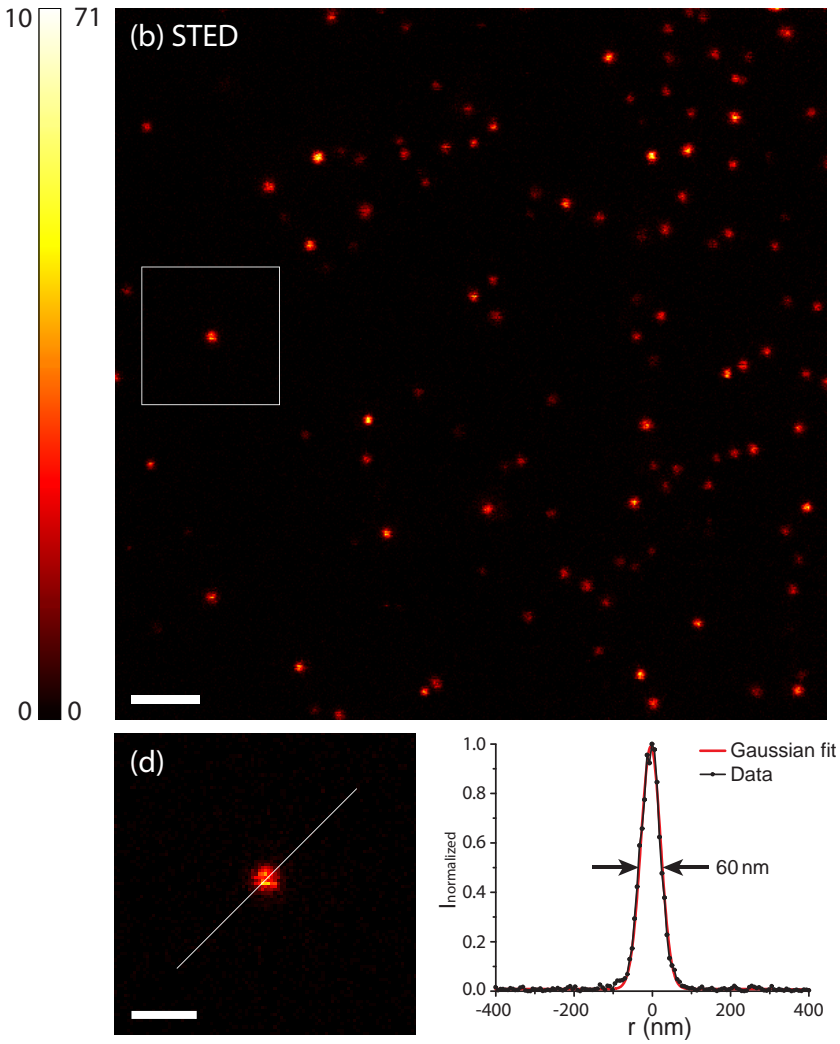

Figure 3.4: Images of $20 \mathrm{~nm}$ crimson beads with confocal and maximum STED resolution (detection pinhole size of 1.2 Airy units of the fluorescence wavelength of $685 \mathrm{~nm}$ )

(a) Confocal image with blurred beads, partly clustered. (b) STED image with sharper, mostly separated beads. Images in (c) and (d) are insets from the white squares in (a) and (b) with a single crimson bead in confocal and STED mode, respectively. Corresponding normalized intensity profiles (averaged over 5 pixels perpendicular to the direction of the profile) along the indicated lines in (c) and (d), respectively. Image resolutions were determined by FWHMs of the Gaussian curve fits. All images are raw data. Detailed imaging parameters are listed in Table A.1. Scale bars: $500 \mathrm{~nm}(\mathrm{a}, \mathrm{b}), 200 \mathrm{~nm}$ $(c, d)$.

For a quantitative analysis of the power dependent resolution scaling of the STED nanoscope, we prepared a more diluted crimson bead sample $\left(1: 10^{6} \mathrm{in}_{2} \mathrm{O}\right)$. Because of the very high photostability of the fluorescent beads, we were able to perform this measurement on the same area of interest with different depletion laser powers. We first acquired a confocal image of $15 \mu \mathrm{m} \times 15 \mu \mathrm{m}$ size and then increased the depletion laser power image by image up to the highest available depletion laser power. The powers of the depletion beam used in this series were $0 \mathrm{~mW}$ (confocal), $14 \mathrm{~mW}, 20 \mathrm{~mW}$, $39 \mathrm{~mW}, 66 \mathrm{~mW}, 96 \mathrm{~mW}, 126 \mathrm{~mW}, 150 \mathrm{~mW}, 169 \mathrm{~mW}$, and $177 \mathrm{~mW}\left(P_{\text {STEDmax }}\right)$. All other imaging parameters were kept unchanged over the whole resolution series.

To analyze the lateral resolution, we used a MATLAB routine ${ }^{28}$ to estimate the FWHM of several beads. In a first step, this routine corrected for drift of bead positions within the image series. The positions of single beads were identified by finding local intensity maxima of the smoothed sum of the four best resolved images $\left(P_{\text {STED }} \geq 125 \mathrm{~mW}\right)$. In the next step, we excluded neighboring beads

${ }^{28}$ Custom-written by Jan Keller-Findeisen, Max Planck Institute for Biophysical Chemistry, Göttingen, Germany 
closer than a certain distance ${ }^{29}$ to each other depending on the applied depletion laser power. Then, for every image of the resolution series, a two-dimensional (2D) circular Gaussian peak function was fitted to each isolated identified bead, and the distribution of the resulting FWHM of the Gaussian peak estimation was obtained.
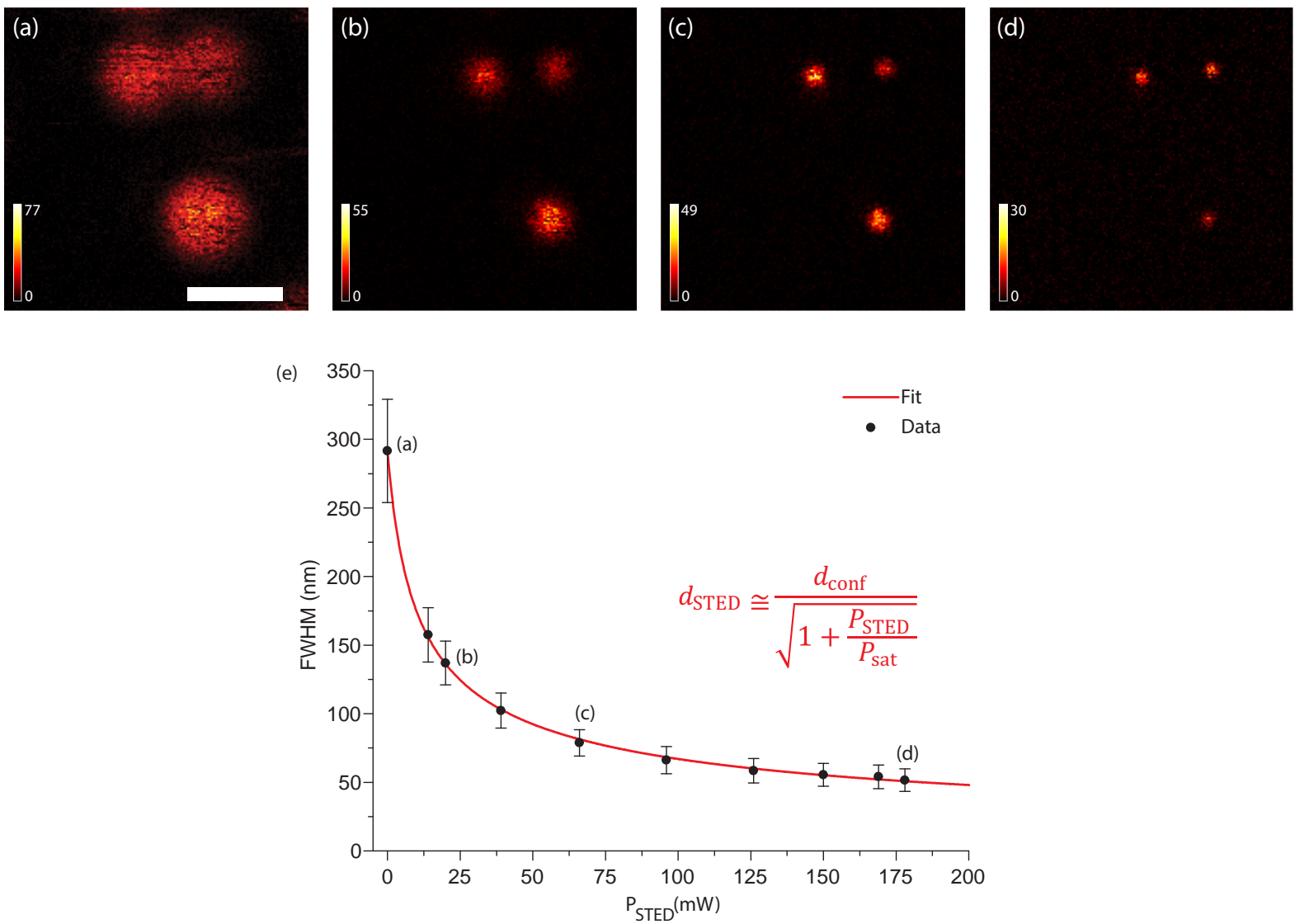

Figure 3.5: Resolution improvement of $20 \mathrm{~nm}$ crimson beads with increasing depletion laser power

(a) Confocal image of three beads. (b-d) STED images of the same sample region. Images were acquired with different depletion laser powers (20 mW (b), $66 \mathrm{~mW}$ (c), $177 \mathrm{~mW}$ (d)). (e) Resolution-scaling graph for the crimson dye. Mean FWHM \pm standard deviation of several isolated beads (at least 55 beads for each data point) as a function of the applied depletion laser power $\left(P_{\text {STED }}\right)$. The resolution-scaling graph followed well the STED resolution formula (fit line with $d_{\text {conf }}=291.4 \mathrm{~nm}$ and $P_{\text {sat }}=5.6 \mathrm{~mW}$ ). At highest available depletion laser power, a lateral resolution of $52 \pm 8 \mathrm{~nm}(n=74$ beads) was achieved in comparison to a FWHM of $292 \pm 38 \mathrm{~nm}$ ( $n=56$ beads) in confocal mode. All images are raw data. Detailed imaging parameters are listed in Table A.1. Scale bar: $500 \mathrm{~nm}$.

The results of the power dependent resolution scaling analysis on $20 \mathrm{~nm}$ fluorescent beads are shown in Figure 3.5. The comparison of the confocal image (Figure 3.5a) and the STED image at highest available depletion laser power (Figure 3.5d) demonstrates the significant resolution increase by using STED nanoscopy. The resolution-scaling graph for the crimson dye quantified the super-resolution capability of the STED nanoscope for different depletion laser powers (Figure 3.5e). The measured lateral resolution was considerably improved with increasing depletion laser power and followed well the STED resolution formula (Equation 1.6) with fitted parameter values of $d_{\text {conf }}=215.4 \mathrm{~nm}$ and $P_{\text {sat }}=5.6 \mathrm{~mW}$. At highest available depletion laser power, the mean FWHM of bead images ( $n=74$ beads) along the lateral direction was $52 \pm 8 \mathrm{~nm}$, a 5.7-fold improvement on the $292 \pm 38 \mathrm{~nm}$ FWHM in the confocal mode ( $n=56$ beads). The saturation power $\left(P_{\text {sat }}\right)$ was extracted out of the resolution-scaling graph and amounted to $5.6 \mathrm{~mW}$ for the crimson dye.

\footnotetext{
${ }^{29}$ Minimum distance of $500 / 400 / 300 / 200 \mathrm{~nm}$ at $0-14 / 15-39 / 40-125 / 126-177 \mathrm{~mW}$ applied depletion laser power, respectively.
} 
As a side note, the finite size of the beads must be considered in the calculation of the resolution when estimating the FWHM of the PSF if both values are similar (Harke, 2008). Nevertheless, this was not necessary here because the measured bead image PSF was still considerably larger ( 2.6 times) than the bead size.

\section{Resolution scaling for the SiR-Halo dye}

Since the STED resolution is determined by the applied depletion laser intensity as well as the saturation intensity of the fluorophore (see Equation 1.4), we were interested in identifying the resolution scaling of the custom-built STED nanoscope for the live-cell compatible fluorescent dye SiR (Lukinavičius et al., 2013), most frequently used in our in vivo studies. Therefore, we estimated its specific resolution by analyzing the thickness of single tubulin filaments in living cells, measuring $24 \mathrm{~nm}$ in diameter (Lodish et al., 2000).

For specific labeling, we transfected Vero cells with $\beta$-tubulin-HaloTag ${ }^{30}$ fusion protein. 24 hours after transfection, cells were incubated with $1 \mu \mathrm{M}$ SiR-Halo dye for 20 minutes at growth conditions $\left(37^{\circ} \mathrm{C}\right.$, $5 \% \mathrm{CO}_{2}$ ), followed by 20 minutes washing with pre-warmed $37^{\circ} \mathrm{C}$ HDMEM (pH7.4, DMEM lacking Phenol Red, 10 mM HEPES, 1\% penicillin, 1\% streptomycin) under growth conditions. STED imaging was performed at room temperature in HDMEM by using the STED nanoscope. Due to photobleaching, we were not able to perform the measurements with different depletion laser powers on the same area of interest. For every depletion laser power, we acquired an image of about 170 to $490 \mathrm{\mu m}^{2}$ size from the tubulin network within an individual cell. The best signal-to-noise ratio was achieved at imaging regions above or below the cell nucleus. The power of the STED beam was varied from $0 \mathrm{~mW}$ (confocal), $14 \mathrm{~mW}, 20 \mathrm{~mW}, 39 \mathrm{~mW}, 66 \mathrm{~mW}, 96 \mathrm{~mW}, 126 \mathrm{~mW}, 150 \mathrm{~mW}, 169 \mathrm{~mW}$, to $177 \mathrm{~mW}\left(P_{\text {STEDmax }}\right)$ within the resolution series. The excitation power was individually adjusted for the imaging series to obtain a decent image contrast, all other imaging parameters were held constant (see Table A.1).

To analyze the lateral resolution, we used implemented tools of the software products ImSpector and Origin to estimate the average FWHM of 10 separated tubulin filaments for every image of the resolution series. We fitted a 1D Gaussian peak function to the intensity profiles (intensity profile was averaged over 10 pixels along the direction of the filament) across each tubulin filament, and calculated the distribution of the resulting FWHMs of the Gaussian curve estimation.

Figure 3.6 presents the experimental data of the power dependent resolution scaling analysis of SiRHalo on tubulin filaments in living Vero cells in a similar manner as shown before for crimson bead samples. The resolution increase of the tubulin network depending on the applied depletion laser power revealed the significant impact of using super-resolution, most obvious by comparison of the confocal (Figure 3.6a) and the STED image at highest available depletion laser power (Figure 3.6d). The resolution-scaling graph for the SiR-Halo dye quantified the super-resolution capability of the STED nanoscope for different depletion laser powers (Figure 3.6e).

The measured lateral resolution was considerably improved with increasing depletion laser power and followed well the STED resolution formula (Equation 1.6). The experimental data was approximated by using $d_{\text {conf }}=295.3 \mathrm{~nm}$ and $P_{\text {sat }}=7.6 \mathrm{~mW}$. A maximum STED resolution of $66 \pm 5 \mathrm{~nm}$ was achieved at the highest available depletion laser power. In contrast, the confocal resolution of the SiR-Halo dye was found to be $300 \pm 28 \mathrm{~nm}$. This represents a lateral resolution improvement of 4.6 at highest available depletion laser power. The saturation power $\left(P_{\text {sat }}\right)$ was extracted out of the resolution-scaling graph and amounted to $7.6 \mathrm{~mW}$ for the SiR-Halo dye.

\footnotetext{
${ }^{30}$ Plasmid was kindly provided by the laboratory of Prof. Yasushi Okada from RIKEN Quantitative Biology Center, Osaka, Japan.
} 

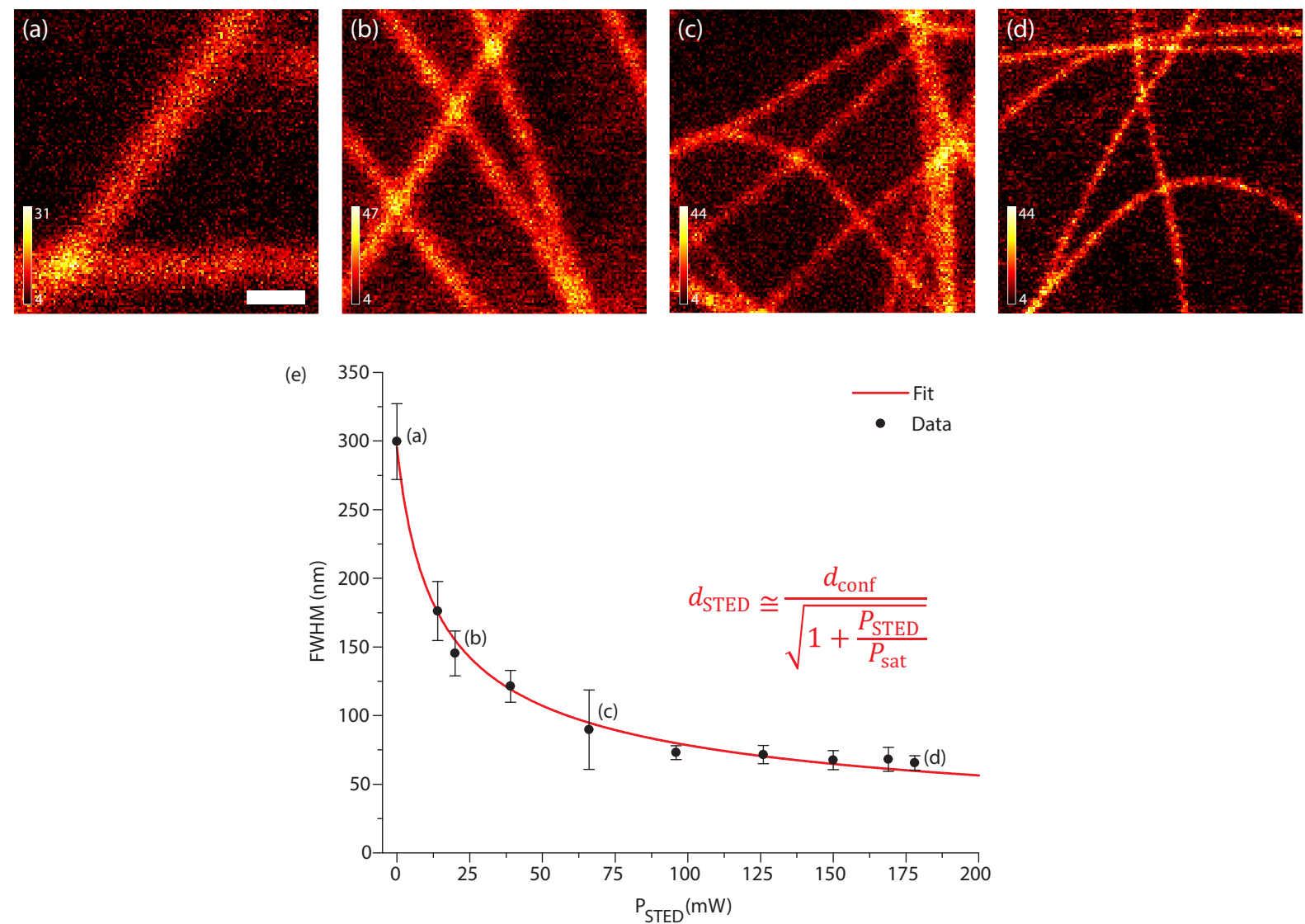

Figure 3.6: Resolution increase in STED nanoscopy of tubulin filaments in living Vero cells, labeled with SiR-Halo Vero cells expressing $\beta$-tubulin-HaloTag fusion protein were live-stained with SiR-Halo (incubation: $1 \mu \mathrm{M}$ for $20 \mathrm{~min}$, followed by 20 min of washing). The tubulin network was live-imaged in confocal mode (a) and with STED nanoscopy of increasing depletion laser power $(20 \mathrm{~mW}$ (b), $66 \mathrm{~mW}$ (c), $177 \mathrm{~mW}$ (d)). (e) Resolution-scaling graph for the SiR-Halo dye. Values represent mean FWHM \pm SD of 10 separated tubulin filaments at each data point as a function of the applied depletion laser power $\left(P_{\text {STED }}\right)$. The resolution-scaling graph followed well the STED resolution formula (fit line with $d_{\text {conf }}=295.3 \mathrm{~nm}$ and $P_{\text {sat }}=7.6 \mathrm{~mW}$ ). At highest available depletion laser power, a lateral resolution of $66 \pm 5 \mathrm{~nm}$ was achieved in comparison to a FWHM of $300 \pm 28 \mathrm{~nm}$ in confocal mode. All images are raw data. Detailed imaging parameters are listed in Table A.1. Scale bars: $500 \mathrm{~nm}$.

The maximum STED resolution for the SiR-Halo dye differed about $27 \%$ from the crimson dye. While the crimson dye is captured in a bead, one has to take into account that the dye SiR-Halo was bound to tubulin using a HaloTag, which slightly increased the diameter of the labeled tubulin structure, the actual size of the HaloTag being $\sim 3-5 \mathrm{~nm}^{31}$. Because the STED resolution is dependent on the applied depletion laser power as well as the saturation intensity of the fluorophore, we used the resolution-scaling graph of the SiR-Halo dye to compare the resolution from data obtained in in vivo studies.

\footnotetext{
${ }^{31}$ We estimated the size of the HaloTag based on recently published x-ray crystal structure data (Liu et al., 2017).
} 


\subsubsection{Summary}

As a part of this thesis, a compact and stable far-red two-color STED nanoscope was designed and constructed for in vivo imaging in brains of mice. With particular consideration given to stability, we have developed a new design concept for an upright in vivo STED nanoscope. It consisted of different modules (one depletion laser, multiple excitation lasers, STED unit, microscope stand, and detection), and the size of the mechanically stabilized STED unit was minimized by reducing the optical beam path lengths. Characteristics of the custom-built setup included a compact, upright two-color STED configuration for the far-red spectral range with a third color in the green-yellow spectrum at confocal resolution, flexible wavefront modulation and aberration correction of the depletion beam with an SLM, beam-scanning with a maximum field-of-view of $130 \mu \mathrm{m} \times 130 \mu \mathrm{m}$ with the Quadscanner, a 1.3 NA glycerol objective lens with aberration correction collar, and a robust and decoupled translational stage for $x y$-scanning of large and heavy objects.

Overall, the custom-built STED nanoscope demonstrated superb optical performance. PSF measurements showed that the intensity distributions of depletion and excitation beams were well aligned without prominent optical aberrations. More specifically, the depletion beam exhibited the desired symmetric doughnut-shaped intensity distribution with a centered, almost zero valued singularity. The temporal alignment of depletion, excitation, and time-gated detection was optimized to achieve the highest possible resolution with enhanced signal-to-noise ratio and no considerably loss of the total fluorescence signal.

For characterization of the setup's imaging capabilities, we used standard available fluorescent beads in fixed samples to estimate the achievable resolution in confocal and STED modes. The dedicated excitation-detection combination for the green-yellow channel operated at a lateral confocal resolution of about $280 \mathrm{~nm}$, and the maximum lateral STED resolution in red/far-red channels was around $52 \mathrm{~nm}$. By using living samples with SiR-Halo dye tagged tubulin, we characterized the fluorophore specific super-resolution capability and achieved a maximum lateral STED resolution of about $66 \mathrm{~nm}$. The performance of the STED nanoscope was stable over weeks with only marginal adjustments of optical and mechanical parts necessary in contrast to less compact systems. With this minimalistic design and an on-top mounted STED unit, we were thus able to overcome the otherwise limited stability of upright STED configurations.

\subsection{Development of labeling strategies for in vivo far-red STED imaging}

For the reliable imaging of nanoscopic structures in vivo, efficient fluorescent labeling is required in addition to a robust STED nanoscope and stable animal preparation. Therefore, a further goal of this thesis was the development of effective labeling strategies for specifically targeting synaptic proteins in living mice. Especially, the use of the recently developed far-red fluorophores for live-cell imaging were of high interest due to their reduced absorbance and scattering in tissue (Shcherbo et al., 2007; Shcherbo et al., 2009; Morozova et al., 2010; Lukinavičius et al., 2013; Grimm et al., 2015; Hense et al., 2015; Butkevich et al., 2016; Lukinavičius et al., 2016; Butkevich et al., 2017). We considered several red and far-red variants of fluorescent proteins (FPs) and fluorescent organic dyes, establishing two labeling strategies with high specificity for both classes of fluorophores, and investigated them for our specific aim. 
The selection of fluorophores for effective labeling was carried out according to the following criteria:

(i) The labeling needed to be non-cytotoxic, live-cell compatible, and highly specific.

(ii) Spectral properties of the fluorophore had to be suitable for the design of the custombuilt STED nanoscope, i.e. the absorption spectrum had to match the excitation laser wavelengths ( $594 \mathrm{~nm}$ and $640 \mathrm{~nm}$ ), the emission spectrum needed to fit the detection channels (red: 611-635 nm; dark-red: 653-717 nm) and had to offer an adequate crosssection for stimulated emission with a depletion wavelength of $775 \mathrm{~nm}$.

(iii) Sufficient brightness of the fluorophore and a strong resistance to photobleaching were desired to achieve maximum STED resolution when applying high depletion laser intensities, and to enable time-lapse imaging and image stack recordings.

(iv) The fluorescent lifetime of the fluorophore had to be relatively long (several nanoseconds) to provide enough time for an effective stimulated emission process.

The labeling strategies based on fluorescent proteins or organic dyes, and their investigations in living mice are presented in the two following sections.

\subsubsection{Labeling based on far-red fluorescent proteins}

The objective of this investigation was to develop a labeling strategy based on red to far-red FPs. Due to the specifications of the custom-built STED nanoscope, we favored FPs that are excitable in the red wavelength spectrum of $594 \mathrm{~nm}$ or $640 \mathrm{~nm}$ and which emit in the far-red spectral range, in order to be compatible with the depletion laser of $775 \mathrm{~nm}$. Table 3.1 lists all available $\mathrm{e}^{32}$ monomeric $^{33}$ far-red FPs with emission maxima above $620 \mathrm{~nm}$, sorted by ascending emission peak.

Table 3.1: Characteristics of red to far-red fluorescent proteins

\begin{tabular}{|c|c|c|c|c|c|c|c|}
\hline FP & $\begin{array}{c}\text { Excitation } \\
\text { peak } \\
\mathrm{nm}\end{array}$ & $\begin{array}{c}\varepsilon_{\text {abs }} \\
\text { at peak } \\
\mathrm{M}^{-1} \mathrm{~cm}^{-1}\end{array}$ & $\begin{array}{c}\text { Emission } \\
\text { peak } \\
\mathrm{nm}\end{array}$ & $\begin{array}{c}\varphi_{\mathrm{fl}} \\
\text { total }\end{array}$ & $\begin{array}{l}\tau_{\mathrm{fl}} \\
\mathrm{ns}\end{array}$ & $\begin{array}{c}\text { Molecular } \\
\text { brightness } \\
\mathrm{M}^{-1} \mathrm{~cm}^{-1}\end{array}$ & $\begin{array}{c}\text { STED } \\
\text { compatible }\end{array}$ \\
\hline mRaspberry ${ }^{[1]}$ & 598 & 86000 & 625 & 0.15 & na & 13000 & na \\
\hline mKate $2^{[2]}$ & 588 & 63000 & 633 & 0.40 & na & 25000 & na \\
\hline mKate $^{[3]}$ & 588 & 45000 & 635 & 0.33 & na & 15000 & na \\
\hline mNeptune2. $5^{[4]}$ & 599 & 95000 & 643 & 0.28 & na & 27000 & na \\
\hline mPlum ${ }^{[1]}$ & 590 & 41000 & 649 & 0.10 & na & 4000 & na \\
\hline mNeptune ${ }^{[5]}$ & 600 & 67000 & 650 & 0.20 & na & 13000 & na \\
\hline mNeptune $2^{[4]}$ & 599 & 89000 & 651 & 0.24 & na & 21000 & na \\
\hline $\operatorname{tagRFP657^{[6]}}$ & 611 & 34000 & 657 & 0.10 & $1.4^{[7]}$ & 3000 & $Y_{e}{ }^{[6]}$ \\
\hline mCardinal ${ }^{[4]}$ & 604 & 87000 & 659 & 0.19 & na & 17000 & na \\
\hline mGarnet $^{[7]}$ & 598 & 95000 & 670 & 0.09 & 0.8 & 9000 & $\mathrm{Yes}^{[7]}$ \\
\hline
\end{tabular}

$\varepsilon_{\mathrm{abs}}$ : extinction coefficient; $\varphi_{\mathrm{f}}$ : fluorescence quantum yield; $\tau_{\mathrm{ff}}$ : fluorescence lifetime; brightness is the calculated product of $\varepsilon_{\mathrm{abs}}$ and $\varphi_{\mathrm{ff}}$; na: not available from literature; [1]: Wang et al., 2004; [2]: Shcherbo et al., 2009; [3]: Shcherbo et al., 2007; [4]: Chu et al., 2014; [5]: Lin et al., 2009; [6]: Morozova et al., 2010; [7]: Hense et al., 2015;

Especially for STED nanoscopy, fluorophores with high extinction coefficients, large fluorescence quantum yields, and long fluorescence lifetime are preferred to achieve bright images with enhanced STED resolution and to enable multiple excitation-depletion cycles. The molecular brightness of the listed FPs varies between 3000 to $27000 \mathrm{M}^{-1} \mathrm{~cm}^{-1}$, which is rather low in comparison

\footnotetext{
${ }^{32}$ As of February 2016

${ }^{33}$ Due to its small size in comparison to multimeric states, a monomeric state is favorable to avoid aggregation artifacts and to minimize perturbations of the target protein (Fernandez-Suarez and Ting, 2008).
} 
to that of the enhanced GFP with a brightness of $32000 \mathrm{M}^{-1} \mathrm{~cm}^{-1}$ (Patterson et al., 1997). As could be expected, labeling with far-red FPs may result in (much) dimmer images than with GFP. Regardless of the reduced brightness, these red and far-red FPs might be more favorable to reduce tissue damage, due to their reduced absorbance and scattering (see Section 1.6).

To identify the most suitable far-red FPs for the intended application in living mice, we investigated the STED performance of different FPs with regard to achievable resolution, brightness, and photostability. We selected mNeptune2, mNeptune2.5, tagRFP657, mCardinal, and mGarnet as potential candidates due to their far-red shifted emission peaks above $640 \mathrm{~nm}$, which ensured a better cross-section for stimulated emission with a depletion wavelength of $775 \mathrm{~nm}$. Although mPlum and mNeptune 1 were potential candidates with emission peaks above $640 \mathrm{~nm}$, we excluded them from further investigations due to the lower molecular brightness in comparison to mNeptune2, which also emitted around $650 \mathrm{~nm}$.

\section{Comparison of far-red fluorescent proteins in vitro}

To investigate the STED performance of the five selected FPs (mNeptune2, mNeptune2.5, tagRFP657, mCardinal, and mGarnet), we initially labeled the actin cytoskeleton of cultured rat hippocampal neurons using viral transduction. We utilized viral vectors based on the adeno-associated virus $(\mathrm{AAV})^{34}$ reported to be highly efficient for long-term expression and less cytotoxic in comparison to the Semliki Forest virus (Liljeström and Garoff, 1991; Lundstrom et al., 2000; Peel and Klein, 2000; Ehrengruber et al., 2001; Lundstrom et al., 2003). All viral vectors were designed as a mixture of AAV serotypes $^{35} 1$ and 2 and with the neuron-specific human synapsin promoter (hSyn) (Kügler et al., 2003; Shevtsova et al., 2005) to accomplish a bright labeling and to achieve a specific expression of fusion constructs exclusively in neurons. In order to target filamentous actin (F-actin), one of the major components of the cytoskeleton, we used either the actin-binding peptide Lifeact $^{36}$ or the antigen-binding nanobody Actin-Chromobody ${ }^{37}$ (ChromoTek GmbH, Martinsried, Germany) instead of simply an actin-FP fusion protein, because direct actin fusions are known to impair cytoskeletal functions and dynamics (Riedl et al., 2008).

Two to four weeks old ${ }^{38}$ cultured neurons, grown on glass coverslips, were transduced in their growth media with the respective viruses (see Table 2.1) encoding a far-red FP targeted to either Lifeact or the Actin-Chromobody. To allow sufficient expression of the fusion construct, neurons were incubated for three to five days before imaging. Cultured neurons were imaged in fresh artificial cerebrospinal fluid buffer at room temperature with STED nanoscopy. Using the aforementioned FPs, we resolved the actin cytoskeletal organization in higher detail than using diffraction-limited

\footnotetext{
${ }^{34}$ Other types of viral vectors e.g. lentivirus could not be utilized due to biosafety requirements in the department.

${ }^{35}$ Viral serotypes vary mainly in terms of the target cell specificity and the speed of gene expression, and differ also in the mechanism in which they are taken up into the cell (Nassi et al., 2015).

${ }^{36}$ Lifeact is a small peptide with only 17 amino acids, which binds to either free globular actin (G-actin) or filamentous actin (F-actin). Due to its small size and weak-binding affinity, it does not affect cytoskeletal functions and dynamics. The binding affinity of Lifeact to F-actin is lower in comparison to G-actin and may impair signal-to-noise ratio due to a constant background of labeled free G-actin in the cytosol (Riedl et al., 2008).

${ }^{37}$ Chromobodies are a fusion product of an FP with the antigen-binding domain of heavy-chain antibody (Hamers-Casterman et al., 1993) $\left(\mathrm{V}_{\mathrm{H}} \mathrm{H}\right)$ from Camelidae (Rothbauer et al., 2006). Due to a molecular mass of $\sim 13 \mathrm{kDa}$ and a size of approximately $2 \mathrm{~nm} \times 4 \mathrm{~nm}, \mathrm{~V}_{\mathrm{H}} \mathrm{Hs}$ are the smallest intact naturally occurring antibodies devoid of light chains, often referred to as nanobodies (Muyldermans, 2001). By genetically fusing nanobodies with FPs, endogenous antigens can be visualized in living cells (Rothbauer et al., 2006).

${ }^{38}$ After 14 days in vitro, neurons are classified as mature and typically have formed a large number of dendritic spins (Dotti et al., 1988; Papa et al., 1995; Kaech and Banker, 2006).
} 
microscopy (data not shown). Compared to the other fluorophores, mNeptune2.5 and mGarnet were dimmer and less photostable. Therefore, we excluded the two FPs mNeptune2.5 and mGarnet for further considerations.

\section{Comparison of far-red fluorescent proteins in vivo}

Following these preliminary tests in cultured neurons, we were interested in testing the remaining three far-red FPs (mNeptune2, mCardinal, tagRFP657) in an in vivo environment and in identifying the best candidate(s) for STED imaging. For this purpose, we performed viral infections (method: Section 2.2.1) with the respective viruses (see Table 2.1) on C57BL/6N wildtype mice, which then expressed the fusion proteins in neurons of the visual cortex. All viral transductions were targeted to layer 5 by injecting the AAV vector solution at a depth of $\sim 500 \mu \mathrm{m}$ below the cortical surface.

To study the performance of the gene transfer and neuron-specific targeting, we perfused the mice (method: Section 2.2.4) two to four weeks after AAV vector injections. We analyzed the gene expression based on $70 \mu \mathrm{m}$ thick coronal brain slices (method: Section 2.2.4). By using epifluorescence microscopy, we examined the spatial extent of the brain area containing the transduced neurons. We found FP expressing neurons in a limited coronal extent ( 0.8 to $2.8 \mathrm{~mm}$ in width for one injection site) of the visual cortex (see Figure 3.7).

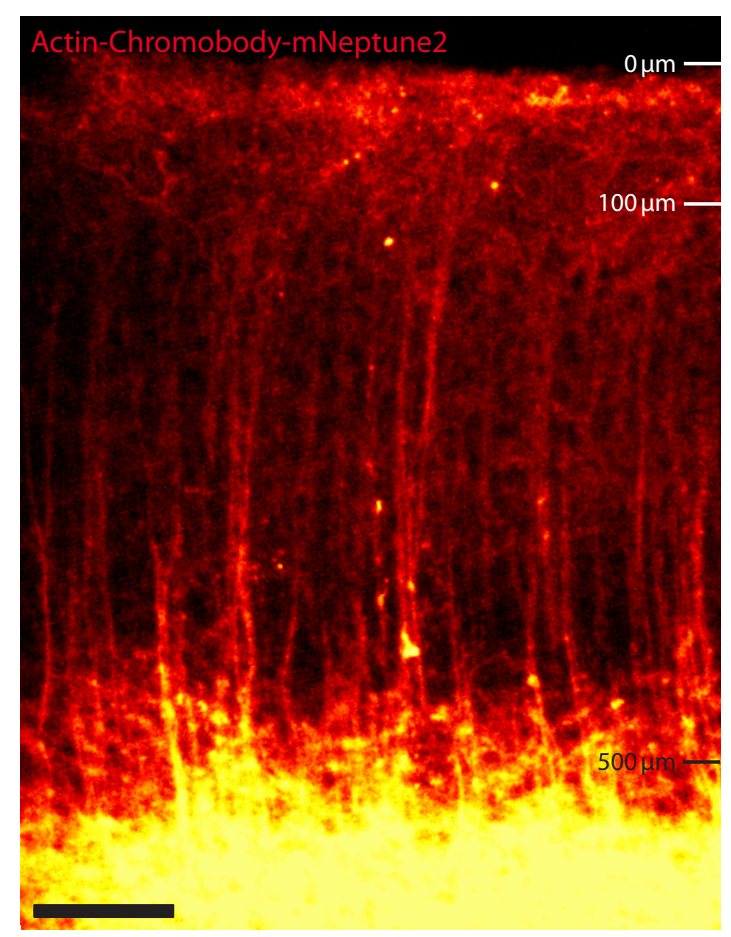

Figure 3.7: Specificity of the AAV-based expression of mNeptune2-labeled Actin-Chromobody in neurons of the visual cortex using the hSyn promotor

Epifluorescence image of pyramidal neurons of the visual cortex (fixed coronal brain section). Cell type-specific transduction of pyramidal neurons within layer 5 led to sufficient labeling of cortical dendrites after two weeks of expression. A limited coronal extent of the neocortex was labeled ( 0.8 to $2.8 \mathrm{~mm}$ in width for one injection site). Apical dendrites were observed to arise from the cell bodies of pyramidal neurons within layer $5(550-700 \mu \mathrm{m}$ below cortical surface), to extend towards the cortical surface, and branch broadly $(0-120 \mu \mathrm{m}$ below cortical surface). Scale bar: $100 \mu \mathrm{m}$. 
Mainly, pyramidal cells of layer $5(550-700 \mu \mathrm{m}$ below cortical surface) and their apical dendrites with fine branches $(0-120 \mu \mathrm{m}$ below cortical surface) were labeled by the AAV transduction. We achieved a homogenous, not too dense, and highly specific labeling of cortical dendrites in layer 1 . This method of AAV injection effectively labeled the cortical dendrites by targeting pyramidal neurons within layer 5 . Therefore, we performed all viral infections within this thesis according to the aforementioned protocol (see Section 2.2.1).

Next, we investigated the STED performance of the three FPs with regard to achievable resolution, brightness, and photostability in living mice. For this, we surgically prepared the mice two to four weeks after viral expression, including a cranial window implantation for STED imaging (see Section 2.2.2). We excited the far-red fluorescent markers with the $594 \mathrm{~nm}$ laser. At the time of these investigations, the STED setup comprised only one far-red detection channel ${ }^{39}$. The applied excitation and depletion laser powers as well as the pixel size and the dwell time were individually adjusted for each FP to find the best compromise of resolution improvement and signal-to-noise ratio. We evaluated genetically encoded far-red FPs in dendrites located 5-25 $\mu \mathrm{m}$ deep in the visual cortex of living mice. We used three mice to assess the performances of each FPs in vivo, focusing our attention primarily on the brightness and possibility of acquiring image stacks. Exemplary images are presented in Figure 3.8. We imaged the actin cytoskeleton of cortical dendrites labeled with the FPs mCardinal (Figure 3.8A), mNeptune2 (Figure 3.8B), and tagRFP657 (Figure 3.8C) in confocal and STED mode. By using STED nanoscopy, we resolved the actin cytoskeletal organization in dendrites and dendritic spines with a good signal-to-noise ratio (low unspecific background apparent) and in higher detail than using diffraction-limited microscopy (resolution improvement by a factor of $\sim 2$ 2.7). As expected, an enrichment of the actin label was mostly found in dendritic spine heads. Depending on the individual photostability of the FPs, we only applied $20-40 \%$ of the available depletion laser power, with $\mathrm{mCardinal}$ and $\mathrm{mNeptune}$ being more resistant to higher depletion laser powers than tagRFP657, which, for this reason, showed the worst performances in terms of resolution. Accordingly, even if a resolution improvement was readily visible in all STED images, the maximum achieved resolution (140-160 nm FWHM) was lower than the nominal resolution of the STED nanoscope, as was investigated on crimson fluorescent bead samples with highest available depletion laser power (see Section 3.2.3).

\footnotetext{
${ }^{39}$ The fluorescence was spectrally filtered by a 690/70 bandpass filter (BP) (F49-691, AHF analysentechnik) and detected with a fiber-coupled APD (SPCM-AQR-13-FC, PerkinElmer, Inc., Waltham, Massachusetts, USA). Both were not components of the most recent configuration of the custom-built STED nanoscope due to later modifications.
} 


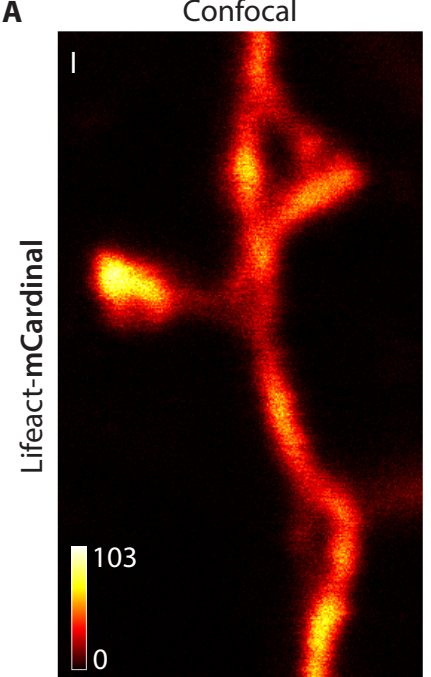

STED
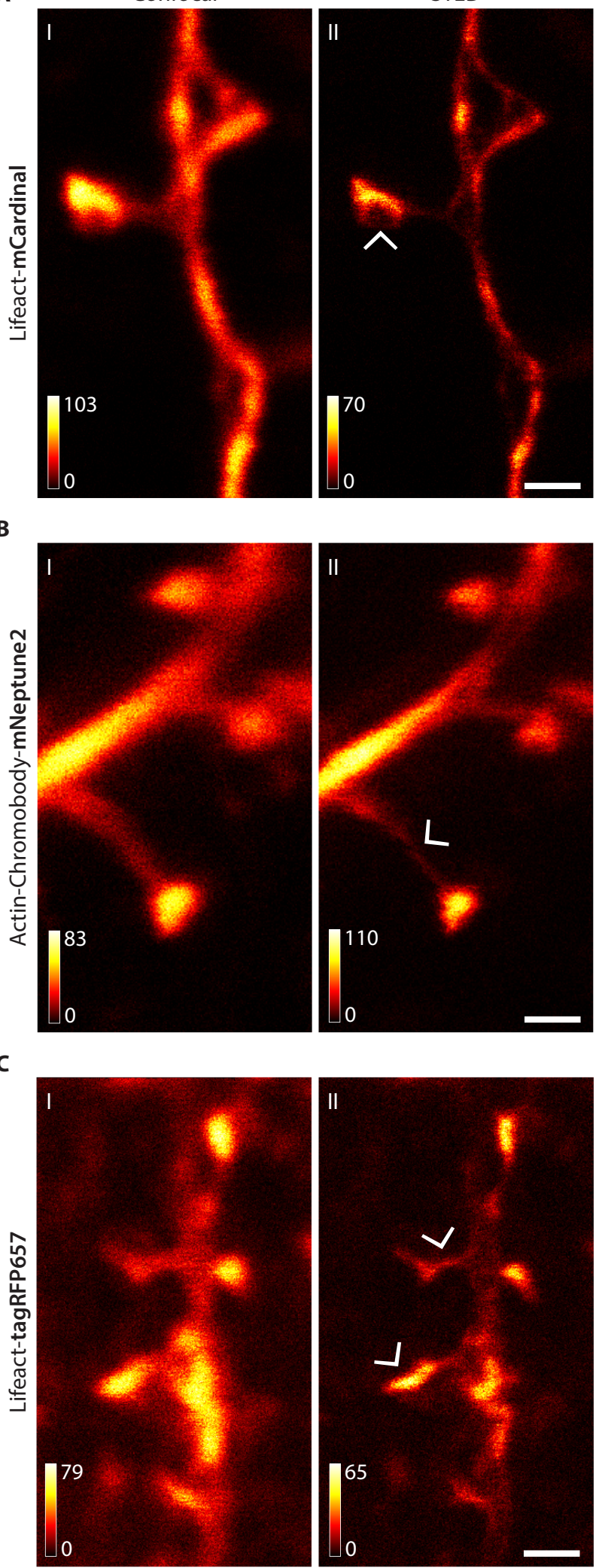
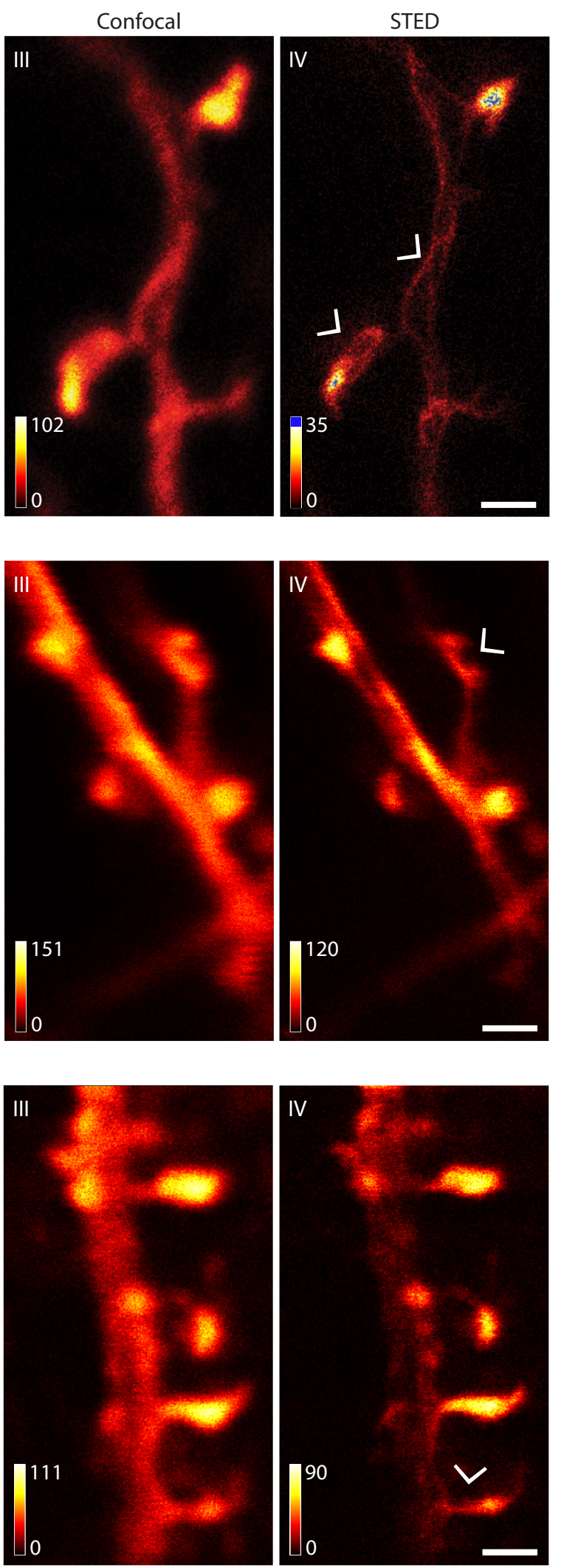

Figure 3.8: In vivo STED imaging of far-red fluorescent proteins in the visual cortex

Confocal (first and third column) and corresponding STED (second and fourth column) images show dendritic actin distribution of (A) Lifeact-mCardinal, (B) Actin-Chromobody-mNeptune2, and (C) Lifeact-tagRFP657. STED nanoscopy revealed the actin cytoskeletal organization and in particular the shapes of the spine heads in much higher detail than confocal microscopy. The arrows indicate details in the STED images, which were not properly resolved in the confocal images. All images are raw data. To emphasize low counts of panel A-IV, the color scale was cut-off at 35 counts per pixel. Detailed imaging parameters are listed in Table A.1. Scale bars: $1 \mu \mathrm{m}$. 
In vivo imaging necessary requires the acquisition of image stacks to appreciate the 3D arrangement of the structure of interest. With this respect, the image quality of tagRFP657 was inferior to mCardinal and mNeptune2 due to a slightly higher background and more bleaching. Therefore, we could not record image stacks, as the fluorophores were bleached before all images could be acquired. In contrast, image stacks were recorded with mCardinal and mNeptune2 labeling (e.g. Figure 3.9), indicating that mCardinal and mNeptune2 were more photostable than tagRFP657 although higher depletion laser powers were used.

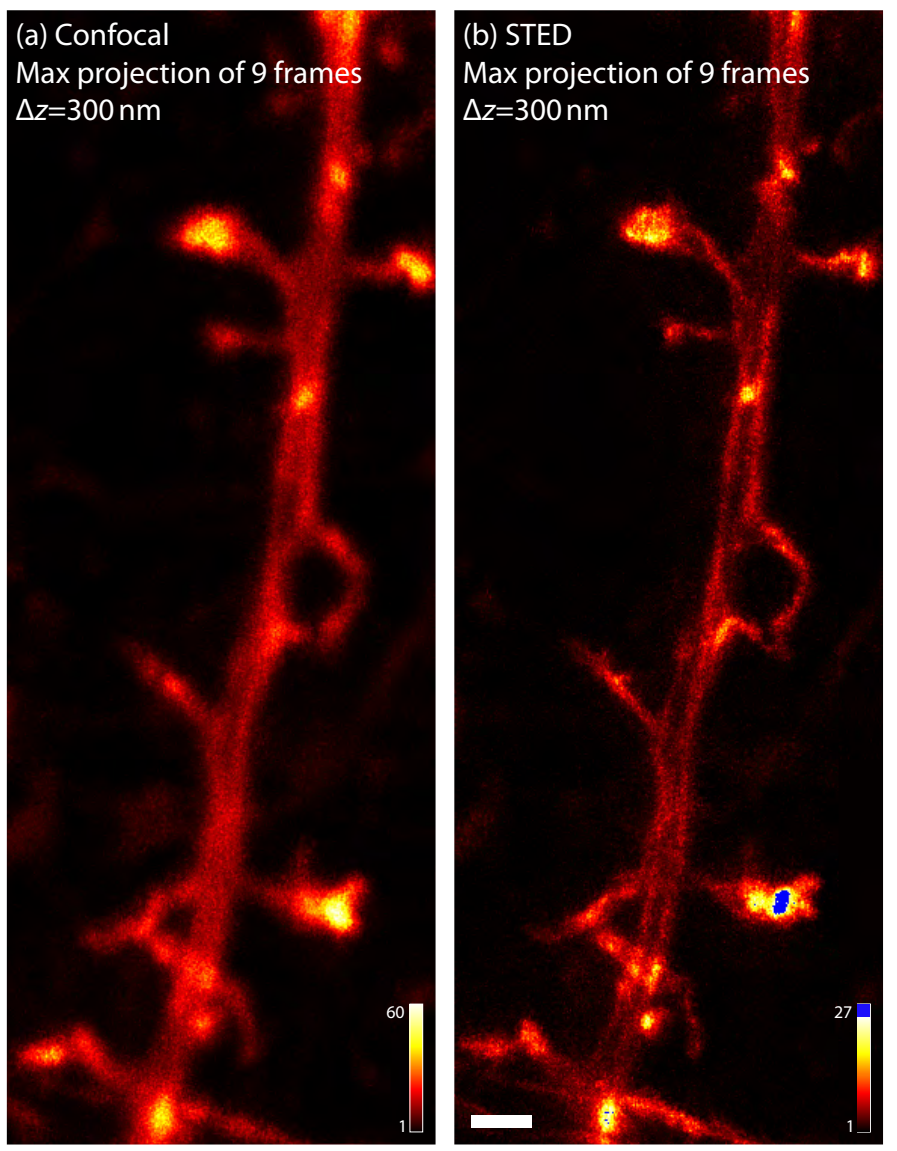

Figure 3.9: In vivo STED imaging of Lifeact-mCardinal in dendrites of pyramidal neurons of the visual cortex (a) Confocal image of a dendritic shaft with several dendritic spines. Actin showed an almost homogenous signal throughout the dendrite with enrichment of the actin label in dendritic spine heads, whereas (b) STED nanoscopy revealed distinct actin filament bundles and better resolved the shape of dendritic spines. Both images are maximum intensity projections (max projection) of stacks of nine $(x y)$ images taken in $300 \mathrm{~nm}$ axial $(z)$ distances. All images were smoothed prior to the maximum intensity projection processing. To emphasize low counts in the STED image, the color scale was cut-off at 27 counts per pixel. Scale bar: $1 \mu \mathrm{m}$.

In conclusion, we demonstrated for the first time the possibility of performing in vivo STED imaging using far-red FPs in combination with a $775 \mathrm{~nm}$ depletion laser. Under our experimental conditions, mCardinal and mNeptune2 showed better performances than tagRFP657 and hence we can anticipate their use for the labeling of synaptic proteins in living mice. Importantly, we did not observe any signs of tissue photodamage throughout the in vivo STED imaging of far-red FPs, further encouraging the use of the far-red spectral configuration proposed for in vivo nanoscopy.

\subsubsection{Labeling based on far-red fluorescent organic dyes}

As a second approach, we developed a labeling strategy based on a genetically encoded tag (see Section 1.4), which covalently binds a membrane-permeable fluorescent organic dye. Since organic 
dyes need to be more photostable for STED nanoscopy as compared to conventional fluorescence microscopy, only a few suitable cell-permeant probes were available. Guided by the specifications of the custom-built STED nanoscope, we focused on organic dyes with emission maxima above $620 \mathrm{~nm}$. Table 3.2 lists the fluorophores that we investigated for our labeling strategy (sorted by ascending emission peaks). The list of fluorophores is by no means exhaustive, but we selected the most widely used dye silicon-rhodamine (SiR; Lukinavičius et al., 2013) as well as promising dyes developed in our department. Note that we considered the fluorescent dye 580CP, even though its emission peak is below $620 \mathrm{~nm}$, since in combination with SiR it enables two-color STED imaging with a single depletion laser at $775 \mathrm{~nm}$ (demonstrated in Butkevich et al., 2016).

Table 3.2: Characteristics of the used far-red fluorescent organic dyes

\begin{tabular}{|c|c|c|c|c|c|c|c|}
\hline Dye & $\begin{array}{c}\text { Excitation } \\
\text { peak } \\
\mathrm{nm}\end{array}$ & $\begin{array}{c}\varepsilon_{\text {abs }} \\
\text { at peak } \\
\mathrm{M}^{-1} \mathrm{~cm}^{-1}\end{array}$ & $\begin{array}{c}\text { Emission } \\
\text { peak } \\
\mathrm{nm}\end{array}$ & $\begin{array}{c}\varphi_{\mathrm{fl}} \\
\text { total }\end{array}$ & $\begin{array}{l}\tau_{\mathrm{fl}} \\
\mathrm{ns} \\
\end{array}$ & $\begin{array}{c}\text { Molecular } \\
\text { brightness } \\
\mathrm{M}^{-1} \mathrm{~cm}^{-1}\end{array}$ & $\begin{array}{c}\text { STED } \\
\text { compatible }\end{array}$ \\
\hline $580 C P^{[1]}$ & $582^{*}$ & 90000 & $607^{*}$ & 0.69 & 3.6 & 62000 & yes $^{[1]}$ \\
\hline $610 C P^{[1]}$ & $609 *$ & 100000 & $634^{*}$ & 0.59 & 3.1 & 59000 & yes $^{[1]}$ \\
\hline $630 \mathrm{GeRH}^{[2]}$ & $631^{*}$ & 61000 & $651^{*}$ & 0.60 & 3.2 & 37000 & $\operatorname{yes}^{[2]}$ \\
\hline $\mathrm{GeR}^{[2]}$ & $634^{*}$ & 97000 & $655^{*}$ & 0.43 & 2.7 & 42000 & yes $^{[2]}$ \\
\hline $640 \mathrm{SiRH}^{[2]}$ & $641^{*}$ & 51000 & $662^{*}$ & 0.42 & 3.2 & 21000 & yes $^{[2]}$ \\
\hline
\end{tabular}

${ }^{*}$ for HaloTag-ligands the spectrum is $5-10 \mathrm{~nm}$ red-shifted; $\varepsilon_{\mathrm{abs}}$ : extinction coefficient; $\varphi_{\mathrm{fl}}$ : fluorescence quantum yield; $\tau_{\mathrm{fl}}$ : fluorescence lifetime; brightness is the calculated product of $\varepsilon_{\mathrm{abs}}$ and $\varphi_{\mathrm{fl}}$; CP: carbopyronine; GeR: germanorhodamine; h: hydroxylated; SiR: silicon-rhodamine; [1]: Butkevich et al., 2016; [2]: Butkevich et al., 2017; [3]: Lukinavičius et al., 2013;

Previous studies have successfully demonstrated the use of the listed far-red dyes in STED nanoscopy of living cells (e.g. actin cytoskeletal organization in rat hippocampal neurons: Lukinavičius et al., 2014; D'Este et al., 2015; vimentin and tubulin filaments in HeLa cells: Butkevich et al., 2016; Butkevich et al., 2017; endoplasmic reticulum, plasma membrane, and Golgi-localized proteins in monkey fibroblast-like kidney cells (COS-7) and HeLa cells: Bottanelli et al., 2016). Therefore, we directly tested the performance of the selected dyes in vivo and, as a benchmark, we labeled the actin cytoskeleton.

\section{Comparison of far-red fluorescent organic dyes in vivo}

For the specific labeling of the actin cytoskeleton in vivo, we established a labeling strategy based on the HaloTag technology (Los et al., 2008), primarily because the listed far-red dyes have already been successfully tested as HaloTag-ligands and because the HaloTag shows the fastest reaction with its ligand (Gautier et al., 2008; Los et al., 2008). Any of the other self-labeling tags would have needed more tests prior to application in in vivo studies. Moreover, the appropriate recombinant AAV vector (rAAV1/2-hSyn-Lifeact-Halo) was readily accessible and tested.

We performed viral infections (method: Section 2.2.1) with both AAV vectors rAAV1/2-hSyn-LifeactHalo and rAAV1/2-hSyn-Lifeact-YFP on C57BL/6N wildtype mice to label the actin cytoskeleton of neurons of the visual cortex. To reach pyramidal neurons of layer 5 , we injected a solution mix of both AAV vectors at a depth $\sim 500 \mu \mathrm{m}$ below the cortical surface. Two to four weeks after the viral infection, we injected the HaloTag-ligand (ligand: Table 2.2; method: Section 2.2.3) into layer 1 of the visual cortex at a depth of $\sim 35 \mu \mathrm{m}$ below the cortical surface. The Lifeact-YFP label served as a reference labeling for the spatial extent of the AAV expression.

For the analysis of gene expression and HaloTag-ligand labeling, we perfused the mice one hour after dye injection and prepared $70 \mu \mathrm{m}$ thick coronal brain slices (method: Section 2.2.4). Using epifluorescence microscopy, we examined the performance of the neuron-specific gene transfer and targeting of the HaloTag-labeling. We found that AAV-based, cell type-specific transduction of pyramidal neurons within layer 5 led to a homogenous, not too dense expression of Lifeact-Halo and 
Lifeact-YFP (reference labeling) in cortical dendrites after two weeks of expression (see Figure 3.10). The injection of the HaloTag-ligand in at least four different locations at a depth of around $35 \mu \mathrm{m}$ within the anticipated Lifeact-HaloTag expression area resulted in a bright labeling of the cortical dendrites one hour after injection. The fluorescent dye penetrated down to a depth of about $110 \mu \mathrm{m}$, so that apical dendritic arborisations of the superficial layer were sufficiently stained. Since the dye diffused more in width than in depth, we identified 4-6 dye injections necessary to achieve a concentrated labeling within a small localized region of the cortex $(\sim 500 \mu \mathrm{m}$ coronal width down to a depth of about $110 \mu \mathrm{m}$ ). The labeling with Lifeact-YFP served as a reference staining to identify the extent of viral expression, and to confirm target specific expression and labeling with the HaloTagligand. In conclusion, the HaloTag-labeling strategy was highly effective to label cortical dendrites with HaloTag-ligands and was therefore used throughout all following experiments, in which fluorescent dyes were used.

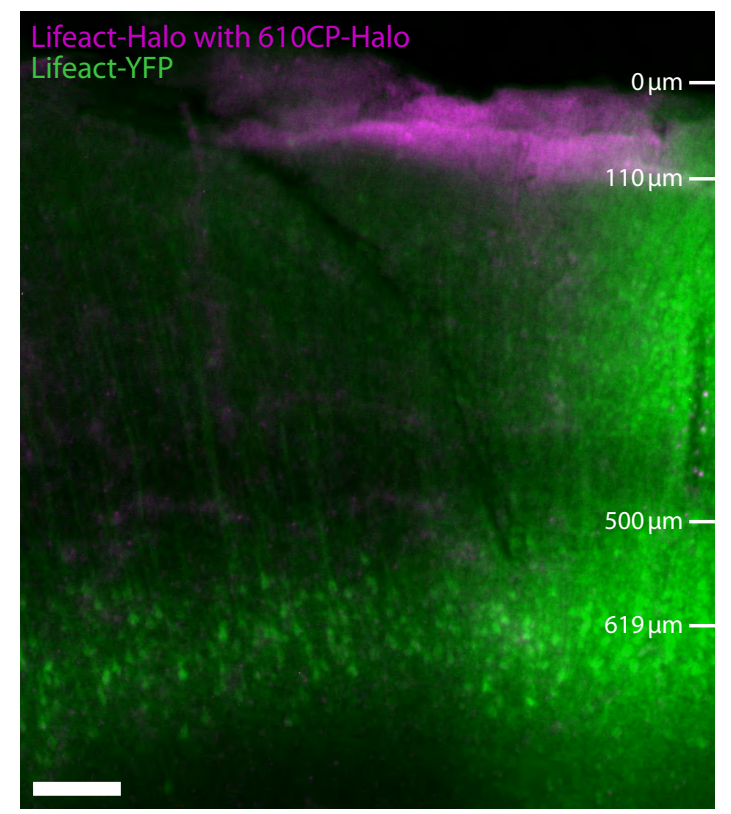

Figure 3.10: Specific and efficient in vivo labeling of Lifeact-Halo in neurons of the visual cortex using the hSyn promotor

Overlay of 610CP-Halo (magenta) and YFP (green) epifluorescence images of pyramidal neurons of the visual cortex (fixed coronal brain section). Cortical pyramidal neurons of layer 5 expressed both rAAV1/2-hSyn-Lifeact-Halo and rAAV1/2-hSyn-Lifeact-YFP. Genetically modified expression was achieved by injecting the respective viruses into layer $5(\sim 500 \mu \mathrm{m}$ below the cortical surface). Two weeks after viral injection, 610CP-Halo-ligand was injected into layer $1(\sim 35 \mu \mathrm{m}$ below the cortical surface) to enable covalent protein labeling in vivo. Lifeact-YFP expressing layer 5 pyramidal neurons were apparent with cell bodies located at depths of 570-670 $\mu \mathrm{m}$ below the cortical surface. Their apical dendrites extended towards the cortical surface and branched broadly $(0-120 \mu \mathrm{m}$ below cortical surface). The labeling of Lifeact-Halo with 610CP-Halo-ligand was localized in a small region of the cortex in the proximity of the injection site, and did not diffuse to the lower cortical layers ( $~ 500 \mu \mathrm{m}$ coronal width down to a depth of about $110 \mu \mathrm{m})$. Scale bar: $100 \mu \mathrm{m}$.

Next, we investigated the STED performance of the six selected far-red fluorescent organic dyes (see Table 3.2) with regard to achievable resolution, brightness, and photostability in living mice. For this, we surgically prepared the mice two to four weeks after viral expression, including a cranial window implantation for STED imaging (see Section 2.2.2). In vivo staining of actin was achieved by injecting 750 nM HaloTag-ligand (ligand: Table 2.2; method: Section 2.2.3) into layer 1 of the visual cortex, as previously described. We performed STED imaging one hour after dye injection. The dyes 580CPHalo, 610CP-Halo, 630GeRH-Halo, and GeR-Halo were excited by the $594 \mathrm{~nm}$ laser, whereas SiR-Halo and $640 \mathrm{SiRH}-\mathrm{Hal}$ were excited by the $640 \mathrm{~nm}$ laser. At the time of these investigations, the STED 
setup comprised only one far-red detection channel ${ }^{40}$. The applied excitation and depletion laser powers as well as the pixel size and the dwell time were individually adjusted for each fluorescent dye to find the best compromise of resolution improvement and signal-to-noise ratio.

We performed imaging in dendrites located 5-25 $\mu \mathrm{m}$ deep in visual cortex of living mice. We used three mice to assess the performances of each fluorescent dye in vivo, focusing our attention primarily on the brightness and possibility of acquiring image stacks. Representative confocal and STED images with different far-red fluorescent organic dye labeling of the actin cytoskeleton in vivo are shown in Figure 3.11. Four of the six selected dyes exhibited bright and uniform labeling of the actin cytoskeletal organization in confocal and STED images (Figure 3.11A: 580CP-Halo, B: 610CPHalo, C: SiR-Halo, and D: 640SiRH-Halo), whereas the stainings with the dyes 630GeRH-Halo and GeRHalo were insufficiently bright (images not shown) and did not allow STED imaging.

By using STED nanoscopy with $20-50 \%$ of the available depletion laser power, we resolved features of the actin distribution in dendrites and dendritic spines that were not visible in diffraction-limited microscopy (resolution improvement by a factor of $\sim 2-2.6$ ). The actin labeling showed a homogenous signal throughout the dendrite with enrichment of actin in dendritic spine heads. The labeling with 610CP-Halo and SiR-Halo provided the best resolution (120-150 nm FWHM) and signal-to-noise ratio. Compared to the other fluorophores, the optical resolution improvement with 580CP-Halo was lower, which was expected due to a smaller cross-section of 580CP-Halo for stimulated emission with the $775 \mathrm{~nm}$ depletion wavelength. In terms of signal-to-noise ratio, the dye 640SiRH-Halo was inferior to the other three dyes due to a substantial nonspecific background fluorescence, which severely degraded the image quality. In contrast, SiR-Halo was superior with almost negligible fluorescent background. The dye SiR is known for its outstanding fluorogenicity (Lukinavičius et al., 2013, Lukinavičius et al., 2014), which means that the ligand is almost non-fluorescent until binding to its target protein (Xue et al., 2015). We found no significant differences between the four dyes in terms of photostability (image stacks were possible for each). For 580CP-Halo, a higher depletion laser power would have been required to achieve a comparable resolution, but this would have affected its photostability. We noticed that STED imaging with the SiR-Halo-ligand required less depletion laser power in comparison to 580CP-Halo, 610CP-Halo, and 640SiRH-Halo, which can probably be attributed to a better cross-section of SiR-Halo for stimulated emission with the $775 \mathrm{~nm}$ depletion wavelength.

Aside from the photophysical behavior, we observed a fluorescence decrease of SiR-Halo 1.5 to 2 hours after dye injection in all experiments $(n=3)$. At the beginning of the STED imaging, the SiR-Halo fluorescence was obvious at several injection spots within the cranial window (see Section 2.2.3). After a certain time, however, the fluorescence had decreased also in areas, where we had not imaged so far. We did not recognize this behavior for the other three dyes and we were not able to reproduce this in control experiments in transfected cultured hippocampal neurons. The observed SiR-Halo fluorescence decrease has (to date) not been reported in literature and could be a targetspecific artifact induced by the SiR-Halo-ligand that destabilizes Lifeact. Despite this peculiar behavior, SiR-Halo and 610CP-Halo were superior to 580CP-Halo and $640 \mathrm{SiRH}-\mathrm{Halo}$ in terms of resolution and fluorogenicity. Therefore, we primarily focused on these dyes for labeling a synaptic protein in living mice (see Section 3.4). We also used 580CP with the aim of performing two-color in vivo imaging in combination with $\mathrm{SiR}$ in the future.

\footnotetext{
${ }^{40}$ The fluorescence was spectrally filtered by a 690/70 BP (F49-691, AHF analysentechnik) and detected with a fiber coupled APD (SPCM-AQR-13-FC, PerkinElmer). Both were not components of the most recent configuration of the custom-built STED nanoscope due to later modifications.
} 
Concluding, we established a surgical and labeling protocol that permits the robust and effective labeling of overexpressed proteins in vivo, and successfully performed nanoscopy experiments on the visual cortex of living mice. We identified a palette of red and far-red HaloTag probes, which can be used for in vivo STED nanoscopy at $775 \mathrm{~nm}$, enabling single and multi-color imaging.
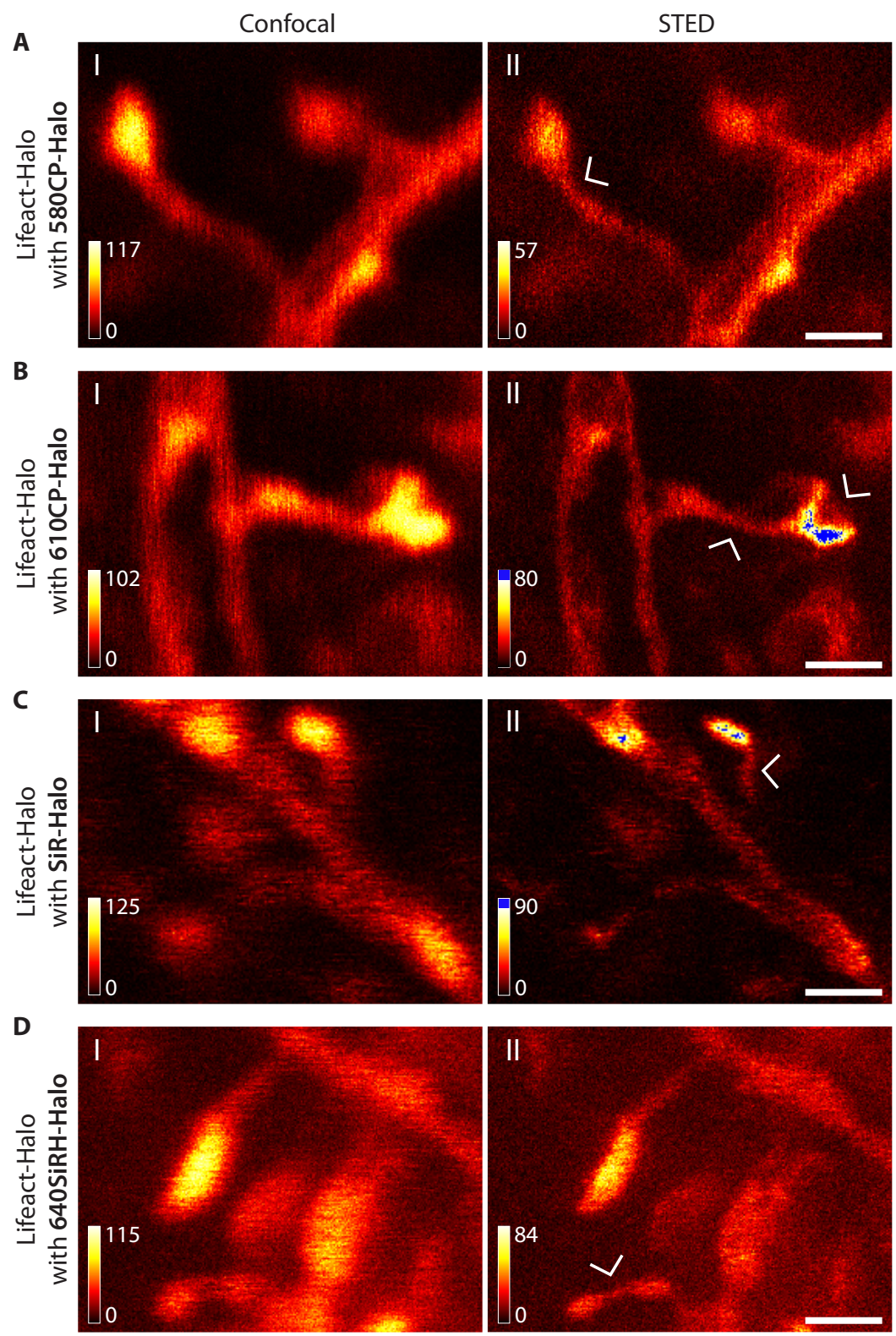

Figure 3.11: HaloTag-labeling of actin cytoskeletal organization in dendrites of the visual cortex in living mice

Confocal (left column) and STED (right column) images of the actin cytoskeleton in dendrites of layer 5 pyramidal neurons, expressing a Lifeact-HaloTag fusion protein (by AAV-mediated infection) and stained with 580CP (A), 610CP (B), SiR (C), and 640SiRH (D) HaloTag-ligands (750 nM). In the STED images, the actin organization and in particular the shapes of the dendritic spine heads were resolved in much higher detail than in confocal images. The arrows indicate details in the STED images, which were not properly resolved in the confocal images. All images are raw data. To emphasize low counts of panels B-II and C-II, the color scale was cut-off at 80 or 90 counts per pixel, respectively. Detailed imaging parameters are listed in Table A.1. Scale bars: $1 \mu \mathrm{m}$. 


\subsubsection{Summary}

Choice of fluorophore and labeling approach are key factors for successful imaging of nanoscopic structures in vivo. Non-toxic fluorescent markers must provide bright images with low background fluorescence and consistent optical properties. Particularly for super-resolution imaging, the fluorescent label needs to be extremely photostable and resistant to high laser intensities to facilitate maximum resolution. Therefore, we developed two labeling strategies based on FPs or organic dyes for in vivo STED nanoscopy with high specificity. We investigated the performance of several far-red variants of FPs and fluorescent organic dyes with emission maxima above $620 \mathrm{~nm}$ in the visual cortex of living mice via overexpression of the protein of interest via AAV infection. Two methodologies, each using different proteins genetically fused to the protein of interest, were employed for labeling: (i) a direct labeling method via FP fusion and (ii) an indirect method with a fused HaloTag-enzyme to be subsequently covalently labeled by intracortical injection of a fluorescent HaloTag-ligand.

Initially, we tested the gene expression of both labeling strategies in coronal brain slices with epifluorescence microscopy. We found that AAV-based, cell type-specific transduction of layer 5 pyramidal neurons in living mice led to adequate expression of both FPs- and HaloTag-fusion proteins in cortical dendrites after two weeks of viral expression. The extent of the FP-based labeling ( 0.8 to $2.8 \mathrm{~mm}$ in coronal width) was broader than HaloTag-based staining due to limited penetration of the exogenous fluorescent dye ( $\sim 500 \mu \mathrm{m}$ in coronal width, $\sim 100 \mu \mathrm{m}$ in depth). However, both labeling strategies sufficiently stained the apical dendritic arborisations in the superficial layer, which were currently accessible to our microscope.

To investigate the STED performance of far-red fluorophores, we labeled the actin cytoskeleton with different FPs and fluorescent dyes, and studied the imaging capability in terms of achievable resolution, brightness, and photostability in vivo. We evaluated three far-red FPs (mNeptune2, mCardinal, tagRFP657) in the brains of living mice. Using STED nanoscopy, we resolved the actin cytoskeleton labeled with these FPs (coupled to Lifeact or Actin-Chromobody) in vivo with a satisfactory signal-to-noise ratio. STED nanoscopy provided a resolution improvement of $\sim 2-2.7$-fold compared to diffraction-limited microscopy. In terms of resolution and photostability, mNeptune 2 and mCardinal were superior to tagRFP657 and therefore good candidates for in vivo STED nanoscopy at $775 \mathrm{~nm}$. In addition to the FPs, we also investigated the STED performance of six farred fluorescent organic dyes for labeling the actin cytoskeleton in vivo using Lifeact-Halo covalently bound to HaloTag-ligands injected one hour before imaging. Four of the six dyes (580CP-Halo, 610CP-Halo, SiR-Halo, 640SiRH-Halo) exhibited bright, precise labeling of the actin organization by STED nanoscopy (resolution improvement of $\sim 2-2.6$-fold compared to diffraction-limited microscopy), though differences in the image quality of the four dyes were salient. 610CP-Halo and SiR-Halo provided the best resolution and signal-to-noise ratio. However, 580CP-Halo, even if enabling imaging at a lower resolution, showed a specific labeling and is an attractive candidate for a two-color imaging scheme in combination with SiR.

In summary, we identified the two FPs, mNeptune2 and mCardinal, as well as the two dyes, $610 \mathrm{CP}$ and SiR, as the most suitable fluorophores for in vivo far-red STED nanoscopy. The latter group (i.e. the genetically encoded self-labeling tagged proteins stained with small-molecule fluorescent organic dyes) exhibited far superior brightness and photostability compared to FPs. These factors enabled both bright images with high resolution and recordings of image stacks. The latter are essential tools to study dynamics or three-dimensional structures in live-cell imaging. Thus, we decided to use fluorescent dye labeling with $610 \mathrm{CP}$ and SiR for the study of synaptic proteins. 


\subsection{In vivo nanoscale imaging of the postsynaptic density protein 95}

This thesis intended to image for the first time the distribution and substructure of a synaptic protein in vivo by STED nanoscopy. We focused on the postsynaptic density 95 (PSD-95) protein (Cho et al., 1992; Kistner et al., 1993), because it is one of the best-studied and most abundant component of the postsynaptic density (PSD) (Chen et al., 2005; Sugiyama et al., 2005; Cheng et al., 2006; Sheng and Hoogenraad, 2007). As the major scaffolding protein, it is located on the spine head where it anchors neurotransmitter receptors and other postsynaptic membrane proteins, and stabilizes numerous signaling molecules within the PSD (Kim and Sheng, 2004).

\subsubsection{Distribution and nanoscale organization of PSD-95 in living mice}

The purpose of the first experiments was to demonstrate the feasibility of STED nanoscopy for imaging PSD-95 in vivo with far-red fluorescent markers. As mentioned in Section 3.3, two promising labeling strategies were identified to target the synaptic protein in fusion with an FP or a HaloTag, respectively. We focused on the latter primarily due to the superior STED performance of the fluorescent dyes 610CP-Halo and SiR-Halo. Moreover, a transgenic knock-in mouse line expressing PSD-95 in fusion with the self-labeling enzyme HaloTag (PSD95-HaloTag, publication in preparation; see Section 2.2) was recently engineered by the laboratory of Prof. Seth G. N. Grant (Centre for Clinical Brain Sciences, Edinburgh University, Edinburgh, United Kingdom). This mouse line allowed us to study endogenous PSD-95-HaloTag in vivo with the use of fluorescent organic dyes as HaloTagligands.

For these experiments, the labeling approach involved a viral infection of the transgenic PSD95HaloTag mouse either with the rAAV1/2-hSyn-eGFP or rAAV1/2-hSyn-Lifeact-YFP vector for morphological reference labeling in pyramidal neurons in layer 5 of the visual cortex (method: Section 2.2.1). After two to four weeks, the mouse was anaesthetized, and a craniotomy was surgically performed at the viral transduction site (method: Section 2.2.2). To stain endogenous PSD95-HaloTag (see Figure 3.12a), we injected the HaloTag-ligand (610CP-Halo or SiR-Halo) into the first layer of the visual cortex, as previously described (ligand: Table 2.2; method: Section 2.2.3). After preparing a cranial window and setting up the mouse for in vivo imaging, we imaged the distribution of endogenous PSD-95 at a depth of 5-25 $\mu \mathrm{m}$ below the brain surface. Differently from what was observed when imaging Lifeact-Halo (see Section 3.3.2), the quality of the labeling did not decrease over time. The dye SiR-Halo was excited by the $640 \mathrm{~nm}$ laser, whereas the dye 610CP-Halo was excited by the $594 \mathrm{~nm}$ laser. The fluorescence of $610 \mathrm{CP}$-Halo was detected with both the red and the far-red detection channels, the fluorescence of SiR-Halo only with the far-red detection channel (for spectral properties see Table 3.2). For a morphological reference in confocal resolution, we excited either eGFP or YFP with the $473 \mathrm{~nm}$ laser and recorded the fluorescence signal with the green-yellow detection channel. The applied excitation and depletion laser powers as well as the pixel size and the dwell time were individually adjusted for each fluorescent marker to find the best compromise between resolution improvement and signal-to-noise ratio.

Figure 3.12b shows a representative overview of the molecular layer of the visual cortex of a living PSD95-HaloTag mouse in confocal mode (overlay of SiR-Halo and YFP fluorescence), which demonstrates the quality of our labeling strategy. The endogenous PSD-95 protein in fusion with the self-labeling enzyme HaloTag was successfully stained by the HaloTag-ligand, and morphological reference labeling was expressed in a high but yet sparse enough number of cortical dendrites. The image overlay revealed, as expected, that the endogenous PSD-95 appears in arrangements of various dimensions and is localized in the dendritic spine heads. However, not all labeled PSD-95 
arrangements colocalized with a corresponding eGFP- or Lifeact-YFP-labeled synapse. This is due to the transduction efficiency of the virus-mediated gene transfer, which is not affecting all neurons, while PSD-95-HaloTag is expressed in every neuronal cell.

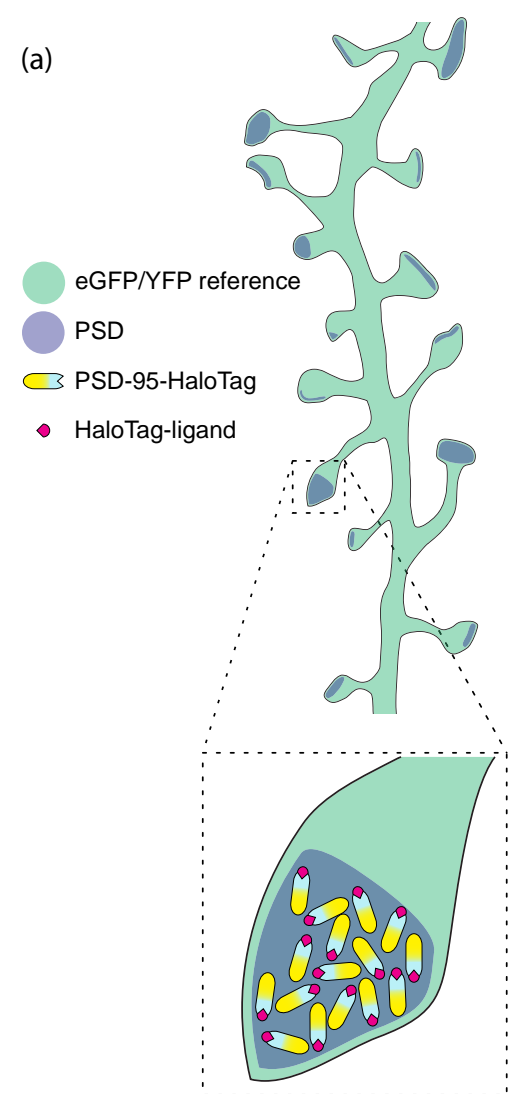

PSD-95-HaloTag with SiR-Halo Lifeact-YFP

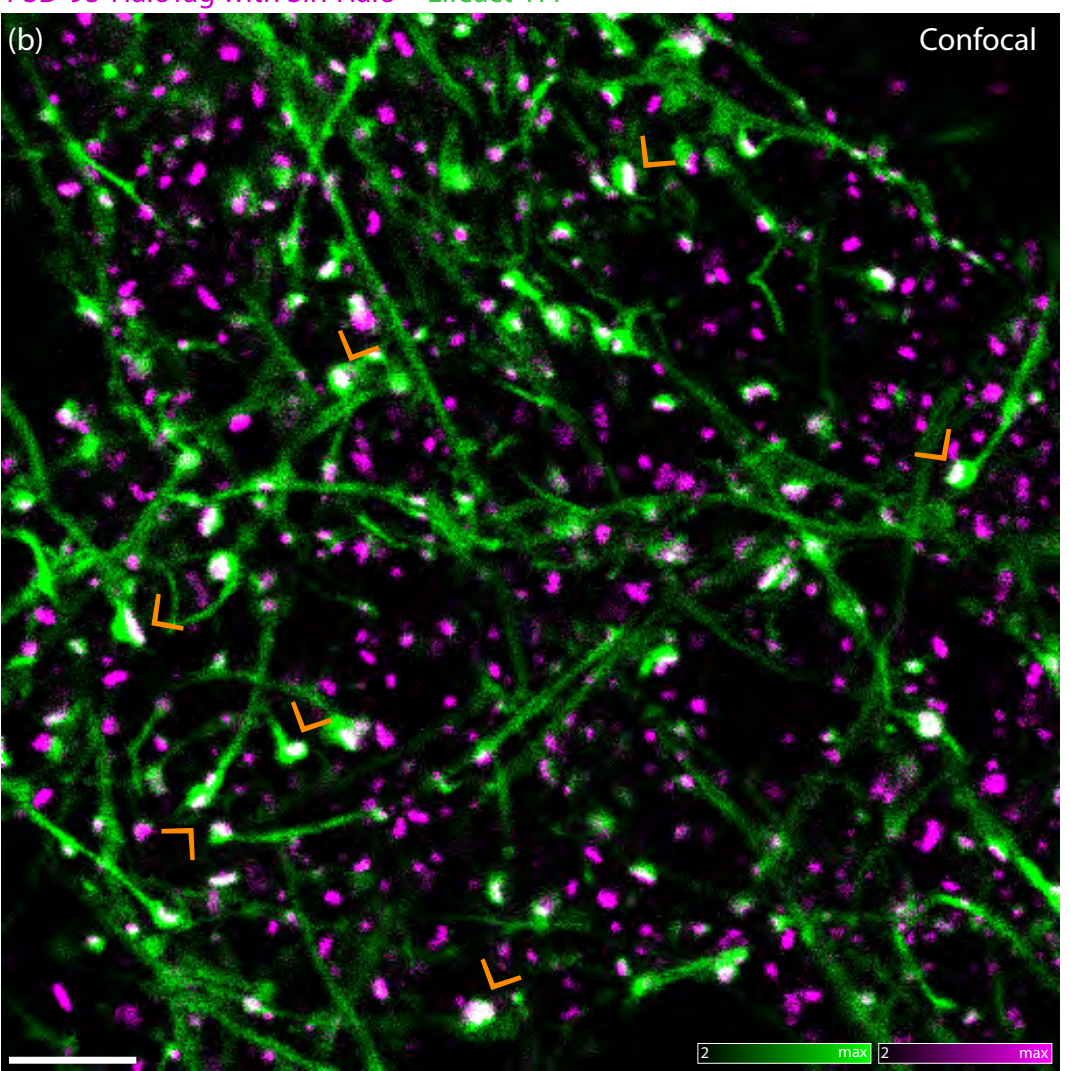

Figure 3.12: In vivo imaging of endogenous PSD-95 in the visual cortex

(a) Strategy for co-labeling PSD-95 and the dendritic morphology. Transgenic PSD95-HaloTag mice were transduced either with the viral vector rAAV1/2-hSyn-eGFP or rAAV1/2-hSyn-Lifeact-YFP two to four weeks before the STED imaging experiment. Hence, cortical dendrites were expressing endogenous PSD-95 as a knock-in with the self-labeling enzyme HaloTag and either exogenous cytoplasmic eGFP or Lifeact-YFP. PSD-95-HaloTag was stained in vivo with $750 \mathrm{nM}$ Haloligand during surgical preparation. (b) Confocal overlay of PSD-95 (magenta; SiR-Halo) and actin (green; Lifeact-YFP). PSD95 labeling was present in different sizes and distributed throughout the dendritic network in the molecular layer of the visual cortex. The morphological reference labeling with YFP shows the localization of PSD-95 at the dendritic spine heads (examples marked by arrowheads). Detailed imaging parameters are listed in Table A.1. Scale bar: $5 \mu \mathrm{m}$.

From confocal overviews (e.g. Figure 3.12), we identified regions-of-interest with PSD-95 located in eGFP- or Lifeact-YFP-positive dendritic spine heads. We imaged the distribution and structure of PSD-95 with super-resolution in relation to the dendritic morphology (Figure 3.13, Figure A.5-A.13). While the confocal images showed PSD-95 scaffolds as blurred dots or lines of various dimensions, STED nanoscopy resolved the substructures of the PSD-95 organizations with excellent image contrast and low background fluorescence. It became apparent that larger structures were not simply puncta, but rather complex PSD-95 arrangements. Elongated PSD-95 shapes exhibited a thickness of about $85 \mathrm{~nm}$ (Figure 3.13aVI, aVII, d, e; FWHM) with a depletion laser power of $66 \mathrm{~mW}$, which is approximately equal to the achievable resolution of our STED nanoscope. The previously determined resolution capability of the dye SiR-Halo in living Vero cells on tubulin-Halo filaments was $90 \mathrm{~nm}$ at this depletion laser power (see Section 3.2.3). 
PSD-95-HaloTag with SiR-Halo (confocal/STED) Lifeact-YFP (confocal)
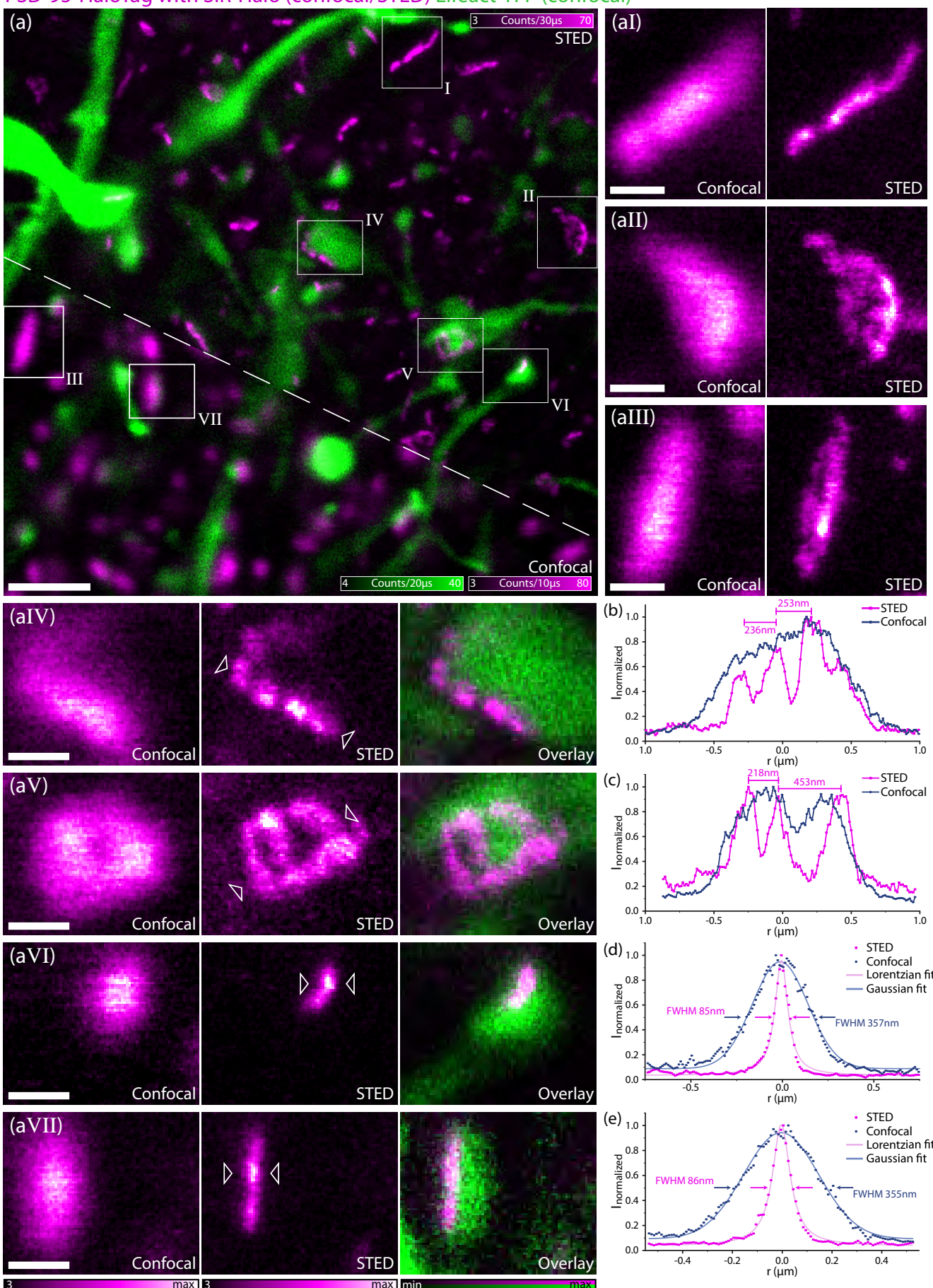

Figure 3.13: In vivo STED nanoscopy of endogenous PSD-95 in the molecular layer of the visual cortex

(a) STED image of endogenous PSD-95-HaloTag stained with $750 \mathrm{nM}$ SiR-Halo-ligand (magenta) in cortical dendrites. Confocal data of PSD-95 is shown in the bottom left corner. Both images were overlaid with the confocal Lifeact-YFP image (green), which served as morphological reference labeling. (al-VII) Insets of regions marked in (a) in confocal mode, STED mode, and the overlay of the STED image with the confocal Lifeact staining. (b-e) Normalized line intensity profiles (five pixel width measured on raw data) from tip to tip of the arrowheads in the STED images (aIV-VII). Gaussian (for confocal data) and Lorentzian fits (for STED data) revealed distances and thickness of features (FWHM). All images were smoothed with a one pixel wide 2D Gaussian filter. Detailed imaging parameters are listed in Table A.1. Scale bars: $2 \mu \mathrm{m}(\mathrm{a}), 500 \mathrm{~nm}$ (al-VII). 
With our in vivo STED imaging approach, we resolved the organization of individual PSD-95 with unprecedented resolution. Our results revealed that the substructures of the PSD-95 organizations were highly diverse, which also might be dependent on the orientation of the synapse in the image plane. Therefore, we investigated the morphological diversity of PSD-95, and we classified them into three groups depending on the appearance: a) small round- or oval-shaped, b) elongated, and c) complex. Figures 3.14, 3.16, and 3.18 represent image galleries showing examples of PSD-95 arrangements within single spine heads for the different categorized shapes, all acquired in vivo (gallery insets are from supplementary Figures A.5-A.12).

In relatively small dendritic spines $(<430 \mathrm{~nm}$ spine head diameter as revealed by Lifeact-YFP reference labeling), the organization of PSD-95 was round- or oval-shaped (Figure 3.14; observed in $53 \%$ of the dendritic spines). Confocal images showed punctated shapes of PSD-95 assemblies, which became much smaller in size with STED imaging. We observed mostly compact nanoscale organizations in STED images, not clustered in shape, whereas some elliptical shapes might have consisted of two single round substructures (see (2) and (10) in Figure 3.14). The reference labeling with Lifeact-YFP indicated that the PSD-95 organizations were located within single spine heads.

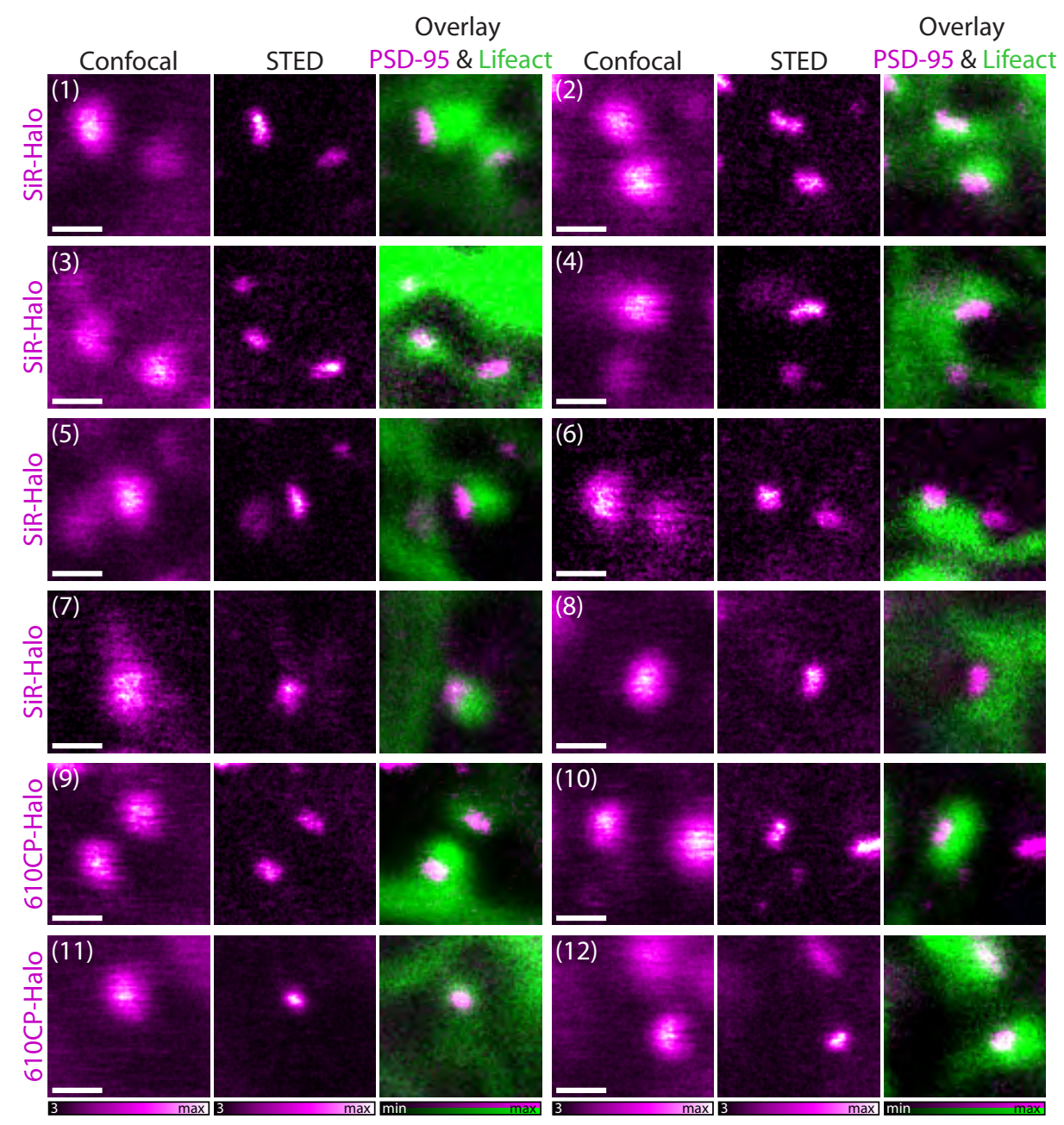

Figure 3.14: Gallery of round- or oval-shaped nanoscale organizations of PSD-95 in vivo

Examples of round- or oval-shaped PSD-95 organizations in confocal and STED modes, as well as overlays of the superresolved PSD-95 organizations (magenta) with the confocal images of Lifeact-YFP (green). Endogenous PSD-95-HaloTag was labeled with the SiR-Halo-ligand (1-8) or with the 610CP-Halo-ligand (9-12). (1-3) are insets from supplementary Figure A.5, (4-5) are insets from supplementary Figure A.6, (6-7) are insets from supplementary Figure A.8, (8) is inset from supplementary Figure A.9, (9-10) are insets from supplementary Figure A.11, (11-12) are insets from supplementary Figure A.12. All images were smoothed with a one pixel wide 2D Gaussian filter. Scale bars: $500 \mathrm{~nm}$. 
To characterize the size of the round- or oval-shaped structures, we measured the lengths of the shortest and longest axes on line intensity profiles along the respective axis. The sizes were determined as FWHMs of the Lorentzian ${ }^{41}$ (for STED data) and of the Gaussian curve (for confocal data) fits on the line intensity profiles. Both the STED and confocal data revealed a wide variability in the size of the round- or oval-shaped structures with short axis ranges of 90-175 $\mathrm{nm}$ for STED and 275-410 $\mathrm{nm}$ for confocal (see Figure 3.15). We exhibited an elliptical shape with an average dimension of $124 \pm 21 \mathrm{~nm}$ (mean \pm standard deviation (SD) short axis) and $223 \pm 46 \mathrm{~nm}$ (mean \pm SD long axis) by STED nanoscopy. Due to the circularity of the STED PSF ${ }^{42}$, this finding indicated a distinct ellipticity of the structure. We expect that they exhibit an ellipticity of at least 2:1 (ratio between long and short axes). In contrast, confocal microscopy revealed an almost round-shaped morphology with an average dimension of $319 \pm 35 \mathrm{~nm}$ (mean \pm SD short axis) and $353 \pm 45 \mathrm{~nm}$ (mean \pm SD long axis), and the elliptical shape of the structure could not identified in the confocal mode. Accordingly, we were able to resolve the small oval organizations of PSD-95 more reliable with STED nanoscopy and observed only a minor fraction of truly round-shaped structures of PSD-95 e.g. (6), (9), and (11) in Figure 3.14.

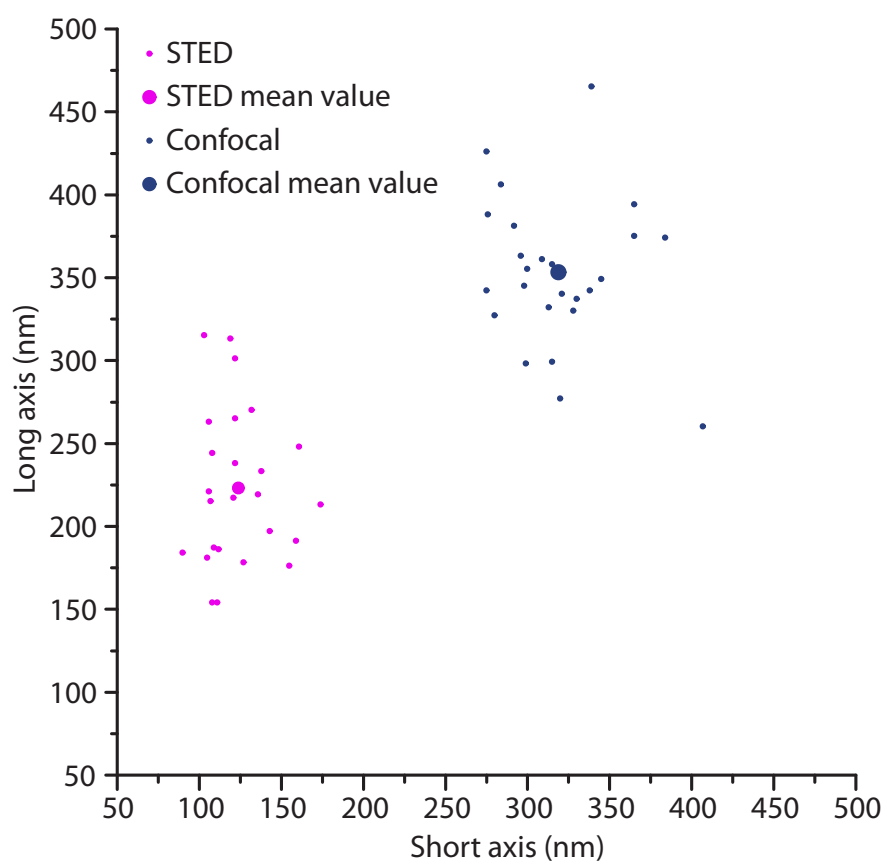

Figure 3.15: In vivo STED nanoscopy reveals an elliptical organization of PSD-95

Size analysis of the round- and oval-shaped nanoscale organization of PSD-95 $(n=25)$. The graph shows the size (short and long axes chosen on STED data) of individual round- and oval-shaped PSD-95 structures for STED (magenta dots) and confocal (blue dots) resolution, determined by fitting Lorentzian (STED data) or Gaussian (confocal data) peak functions to line intensity profiles (five pixel width measured on raw data). Enlarged data points represent mean values for STED and confocal microscopy, respectively.

\footnotetext{
${ }^{41}$ The small features of PSD-95 structures in the STED image, which is a convolution of the object with the PSF of the imaging system, were fitted with a Lorentzian curve to provide a more accurate fit than a Gaussian curve. ${ }^{42}$ Since the image of an object is a convolution of the PSF with the object (see Section 1.1), native structures or shapes can be altered. Here, the actual ellipticity of the object was reduced as a result of the convolution with a circular PSF. The larger the PSF, the greater is the decrease of the apparent ellipticity in the image. Accordingly, confocal microscopy with a large PSF resolved these elliptical objects as circular ones.
} 
The second category of PSD-95 arrangements is the elongated shape, which we observed in $30 \%$ of the dendritic spines (see Figure 3.16). For this category, the confocal images showed elongated or large elliptical PSD-95 organizations, whereas STED nanoscopy resolved the shapes more precisely as thin, elongated bars. PSD-95 appeared mostly continuous in STED images, but sometimes it showed interruptions (see (9) and (10) in Figure 3.16).
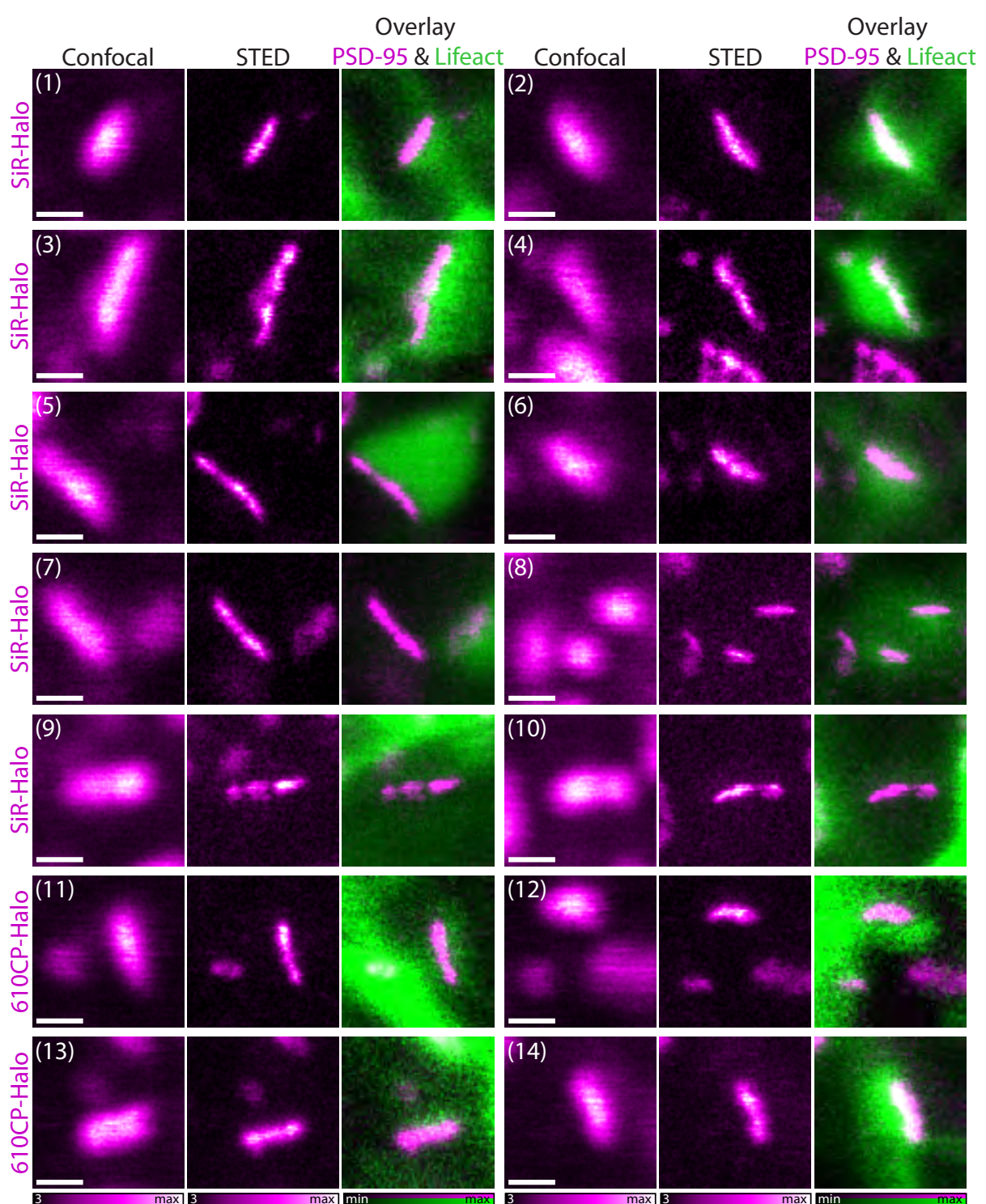

Figure 3.16: Gallery of elongated nanoscale organizations of PSD-95 in vivo

Examples of the elongated PSD-95 organizations in confocal and STED modes, as well as overlays of the super-resolved PSD-95 organization (magenta) with the confocal images of Lifeact-YFP (green). Endogenous PSD-95-HaloTag was stained with the SiR-Halo-ligand (1-10) or with the 610CP-Halo-ligand (11-14). (1-4) are insets from supplementary Figure A.5, (57) are insets from supplementary Figure A.6, (8-10) are insets from supplementary Figure A.7, (11-12) are insets from supplementary Figure A.11, (13-14) are insets from supplementary Figure A.12. All images were smoothed with a one pixel wide 2D Gaussian filter. Scale bars: $500 \mathrm{~nm}$.

Similar to the data shown above from round- or oval-shaped organizations, we performed a size analysis of several elongated nanoscale arrangements of PSD-95. We measured again the sizes of the shortest and longest axes of the elongated shapes by line intensity profiles along the respective axis. For the short axis, we calculated the FWHMs of the Lorentzian (for STED data) and of the Gaussian (for confocal data) curve fits. The extent of the long axis was determined by estimating the FWHMs directly from line intensity profiles (for STED and confocal data). We found that the elongated PSD- 
95 structures varied a lot in length (see Figure 3.17). In STED nanoscopy, we measured a range of 80 $145 \mathrm{~nm}$ (confocal: $275-375 \mathrm{~nm}$ ) for the short axis, whereas the long axis ranged from 375 to $1065 \mathrm{~nm}$ (confocal: 415-1075nm). The elongated structures exhibited an average dimension of $113 \pm 19 \mathrm{~nm}$ (mean \pm SD short axis) and $630 \pm 185 \mathrm{~nm}$ (mean \pm SD long axis) or $322 \pm 27 \mathrm{~nm}$ (mean \pm SD short axis) and $699 \pm 176 \mathrm{~nm}$ (mean \pm SD long axis) for STED or confocal data, respectively.

Depicted by the Lifeact-YFP reference labeling, the dendritic spine heads with elongated PSD-95 organizations seemed to be larger in comparison to synapses with round- or oval-shaped PSD-95 structures. However, a careful assessment of the volume of the spine heads from our data was difficult due to their three-dimensional geometry. Moreover, the elongated formation of PSD-95 was mainly observed from side-views of the synapses.

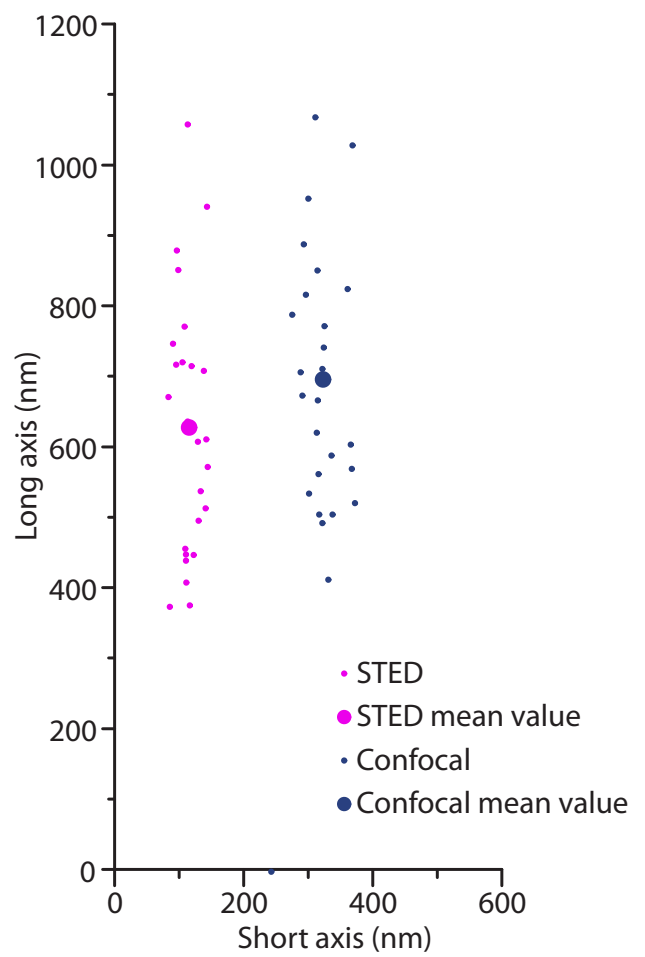

Figure 3.17: Size analysis of the elongated organization of PSD-95

The graph shows the size (short and long axes) of individual elongated PSD-95 structures for STED (magenta dots) and confocal (blue dots) resolution $(n=25)$. Sizes of the short axes were measured by fitting Lorentzian (STED data) or Gaussian (confocal data) peak functions to line intensity profiles (averaged over five pixels of the raw data pixels perpendicular to the direction of the axis) through raw data. Sizes of the long axes were determined by manually measured FWHMs (STED and confocal data) from line intensity profiles (five pixel width measured on raw data). Enlarged data points represent mean values of the short and long axes for STED and confocal microscopy, respectively.

The third category of PSD-95 organizations we observed is a complex (ring-like to oval) substructure with irregular borders and perforations (see Figure 3.18), which were found in $17 \%$ of the dendritic spines. Some of them featured larger gaps fragmenting the morphology. In contrast, confocal images showed blurred and irregular shapes without any major evidence of distinct perforations and substructures. Due to the heterogeneity of these structures, we performed a simple size analysis on the STED data to ascertain their spatial dimensions. To analyze the size of several PSD-95 organizations from Figure 3.18, we measured the lengths of two axes: (i) the length of the longest axis and (ii) the length of the perpendicular orientated axis through the center of the longest side. The sizes determined by estimating the FWHMs directly from line intensity profiles along the respective axis. 


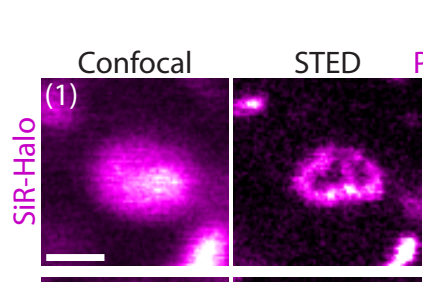

Overlay
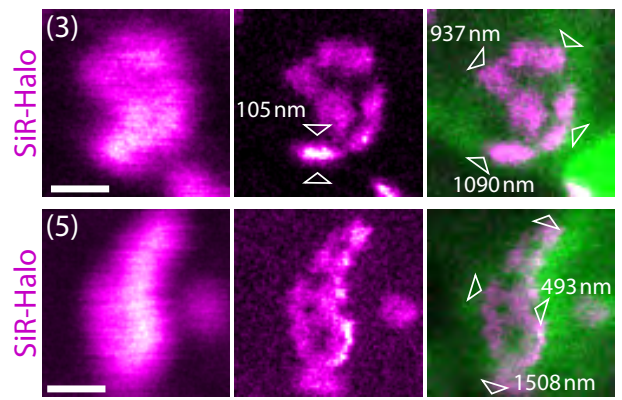

PSD-95 \& Lifeac

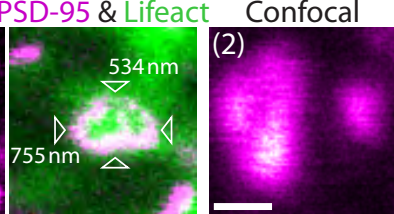

STED PSD-95 \& Lifeact
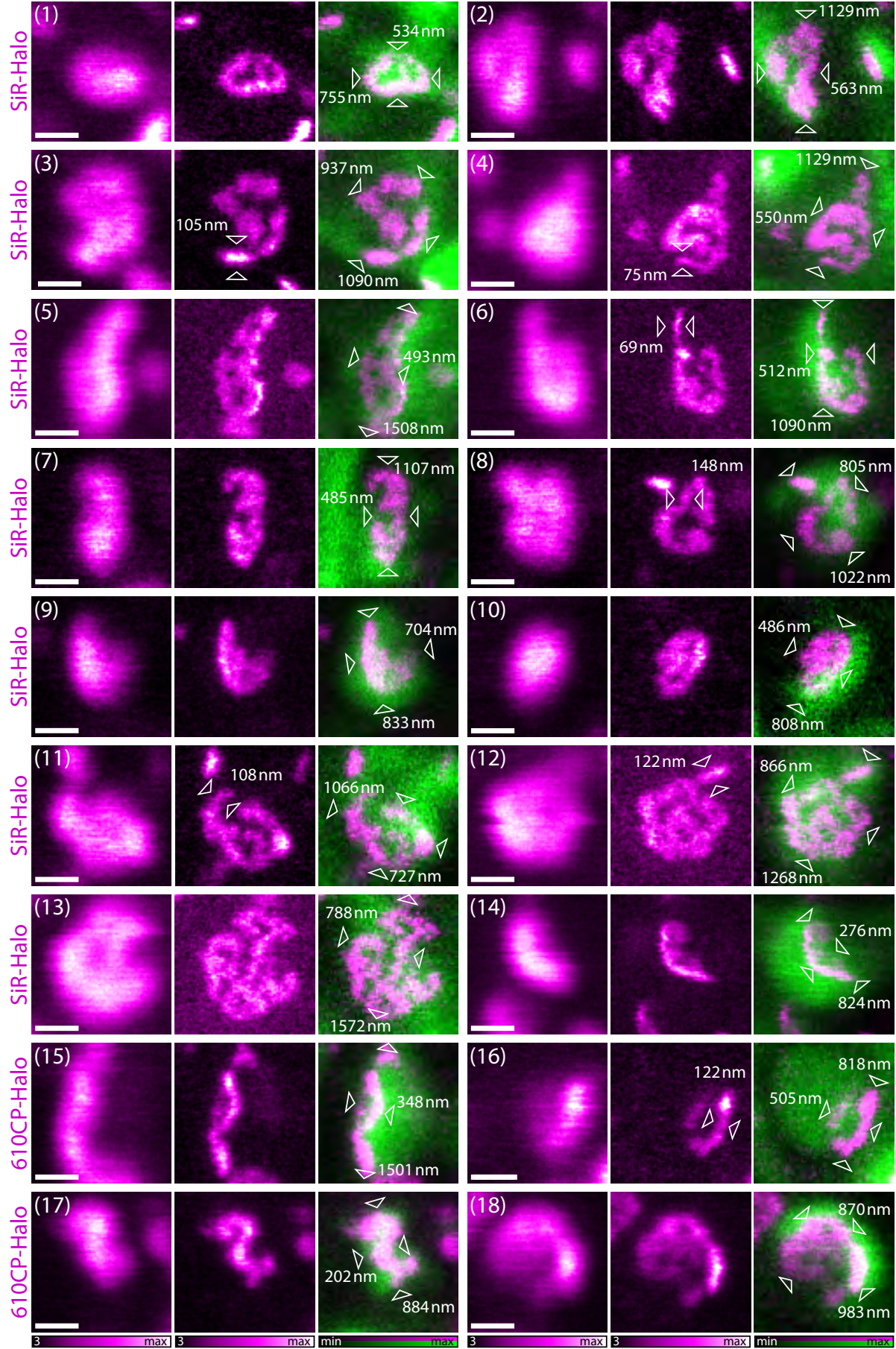

Figure 3.18: Gallery of the complex-shaped nanoscale organizations of PSD-95 in vivo

Examples of the complex-shaped PSD-95 organizations in confocal and STED modes, as well as the overlays of the superresolved PSD-95 organizations (magenta) with the confocal images of Lifeact-YFP (green). Endogenous PSD-95-HaloTag was labeled with the SiR-Halo-ligand (1-14) or with the 610CP-Halo-ligand (15-18). Provided dimension values were measured along the indicated lines (from tip to tip of the corresponding arrowheads), determined by estimating the FWHMs (STED data) directly from line intensity profiles (five pixel width measured on raw data, measured size corresponds to the width at the height of half the maximum of the smallest intensity peak of the profile). Sizes of small features in the STED images were measured along the indicated positions (from tip to tip of the corresponding arrowheads), determined by fitting Lorentzian peak functions to line intensity profiles (five pixel width measured on raw data). (1-2) are insets from supplementary Figure A.5, (3) is inset from supplementary Figure A.6, (4-6) are insets from supplementary Figure A.7, (79) are insets from supplementary Figure A.8, (10-11) are insets from supplementary Figure A.9, (12-14) are insets from supplementary Figure A.10, (15-16) are insets from supplementary Figure A.11, (17-18) are insets from supplementary Figure A.12. All images were smoothed with a one pixel wide 2D Gaussian filter. Scale bars: $500 \mathrm{~nm}$. 
The complex-shaped organizations of PSD-95 varied considerably in size (see Figure 3.18) with an average dimension of $1077 \pm 251 \mathrm{~nm}$ (mean \pm SD long axis) and $592 \pm 210 \mathrm{~nm}$ (mean \pm SD perpendicular orientated axis through the center of the longest axis). Based on the YFP reference labeling, we saw that the complex-shaped structures of PSD-95 tend to occur in the largest spine heads and are en face views of the postsynaptic densities. Next, we measured the size of small features of some nanoscale organizations from the STED images by determining the FWHM of the Lorentzian curve fit of line intensity profiles along the respective axis. We measured a mean width of $107 \pm 28 \mathrm{~nm}$ by STED nanoscopy, whereas this measurement was not even possible with confocal microscopy. Notably, this mean value was in a good agreement with above measured smallest widths of round and elongated PSD-95 morphologies. This might be a hint that small round- or oval-shaped structures could represent the smallest unit of the complex-shaped organizations.

As previously mentioned and in consideration of the eGFP/YFP reference labeling, the appearance of PSD-95 arrangements is dependent on the orientation of the synapse with respect to the focal plane. We classified the PSD-95 structures based on their appearance in 2D images with a low resolution along the optical axis, which then resemble add up projections of the native 3D structure onto the 2D image plane.

PSD-95-HaloTag with SiR-Halo (STED) cytosolic eGFP (confocal)
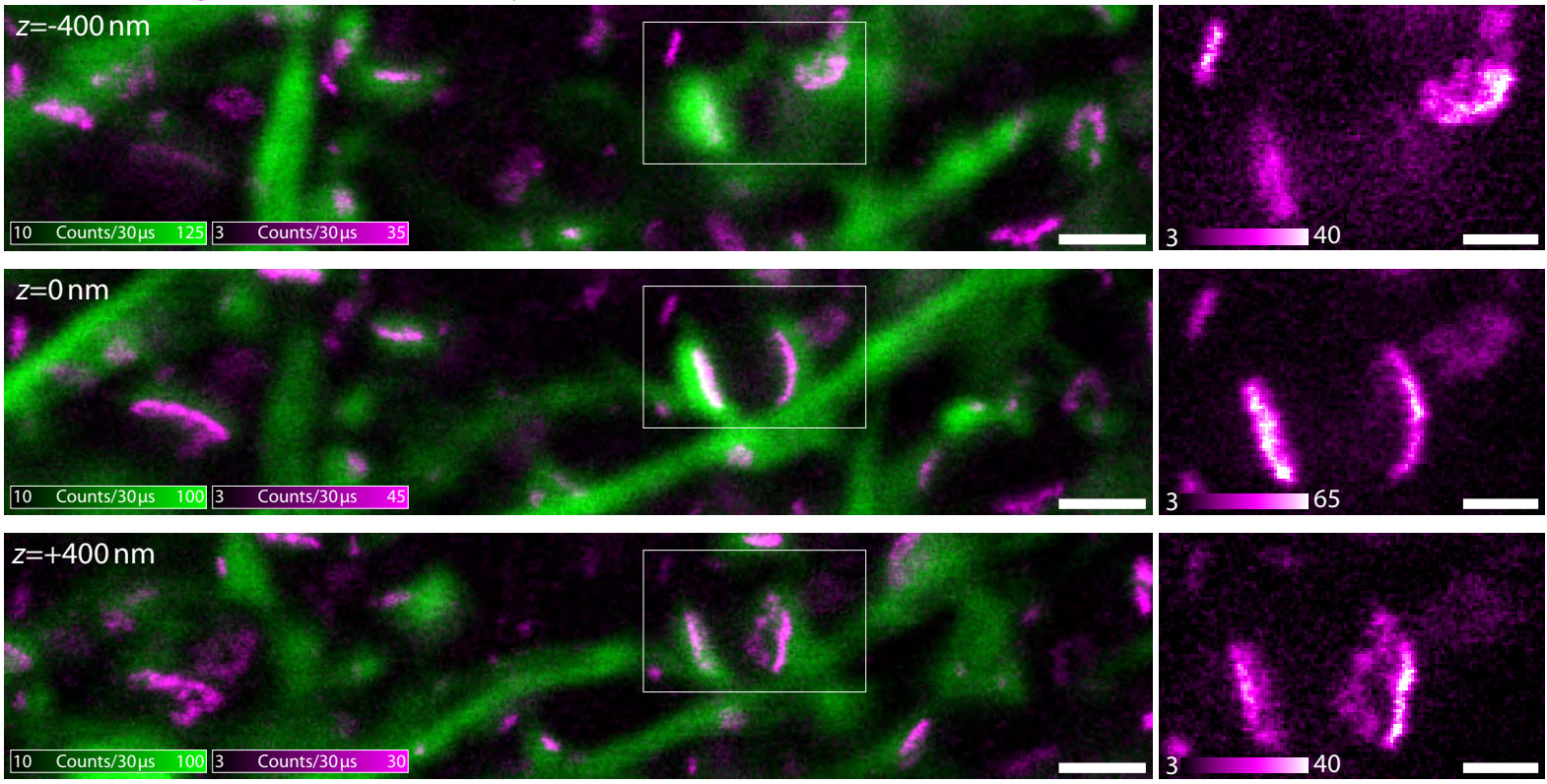

Figure 3.19: Three-dimensional nanoscale organization of PSD-95 in vivo

Organizations of individual PSD-95 dependent on the orientation of the synapse with respect to the focal plane. (Left panel) Overlays of the STED images of PSD-95 (magenta) with the corresponding confocal images of cytosolic eGFP (green) from an axial image series. Endogenous PSD-95-HaloTag was labeled with the SiR-Halo-ligand and imaged at different depths within the molecular layer of the visual cortex of a living mouse. The individual images were recorded as a stack with a $400 \mathrm{~nm}$ axial step size (acquisition order: $z=0, z=-400 \mathrm{~nm}, z=+400 \mathrm{~nm}$, the $z$-axis corresponds to the propagation direction of the incident light). (Right panel) Insets from corresponding STED images of the left panel. Detailed imaging parameters are listed in Table A.1. All images were smoothed with a one pixel wide 2D Gaussian filter. Scale bars: $1 \mu \mathrm{m}$ (overlay), $500 \mathrm{~nm}$ (insets).

To obtain information about both the lateral and the axial organizations of the PSD-95, we recorded image stacks (benefiting from the high photostability afforded by the fluorescent labels). In fact, the image stacks revealed that PSD-95 arrangements in one focal plane were classified by elongated shapes, but exhibited complex and perforated structures in a parallel imaging plane located $400 \mathrm{~nm}$ apart along the $z$-axis (representative example in Figure 3.19). However, we also encountered some 
nanoscale structures, which did not differ in their organization along the $z$-direction. Hence, our data suggest a connection between elongated and complex-shaped structures, which can be dependent on the orientation of the synapse with respect to the focal plane.

In this section, we successfully demonstrated the feasibility of STED nanoscopy for imaging PSD-95HaloTag in vivo with the dyes SiR-Halo and 610CP-Halo, revealing unprecedented features. As mentioned in Section 3.3.2, the dye 580CP-Halo also provided adequate staining of HaloTag-fusion proteins. Therefore, we performed STED imaging studies using 580CP-Halo to label endogenous PSD-95-HaloTag. We found that 580CP-Halo stained PSD-95-HaloTag successfully, whereby STED images (representative example in supplementary Figure A.13) showed lowered resolution in comparison to data acquired with $610 \mathrm{CP}$ - or SiR-Halo labeling. Finally, 580CP can be considered for future two-color in vivo imaging studies due to its favorable fluorescent emission spectrum, which is clearly distinguishable from the SiR emission (but not from 610CP emission).

\subsubsection{Nanoscale rearrangement of PSD-95 in living mice}

The previous section showed that PSD-95 forms diverse nanoscale structures with large variations in shape and size. Next, we investigated whether the arrangement of PSD-95 changes over time or retains a stable morphology. To address this question, we performed time-lapse STED imaging of PSD-95 in living mice.

First, we investigated the PSD-95 organizations on a long-term time scale by recording image stacks at two time points ( 0 and 8 minutes). To represent $2 \mathrm{D}$ images of the three-dimensional organizations, we processed the individual image stacks by generating maximum intensity projections. Projections of three image planes ( $400 \mathrm{~nm}$ axial step size) were sufficient to capture the complete extent of the PSD-95 organization. The preliminary results of this investigation are illustrated in Figure 3.20. When comparing the shapes of the dendritic shafts between the two maximum intensity projections (eGFP reference labeling in Figure 3.20a and b), no significant morphological changes of the dendritic shafts were visible, suggesting that they did not modify during the two acquisition time points. While most of the PSD-95 organizations were rather stable over time (e.g. Figure 3.20 (2) and (4)), other postsynapses exhibited slight changes both of the structure of PSD-95 and the spatial orientation of the dendritic spine heads (Figure 3.20 (1), (3), (5), (6)).

To further examine time-dependent variations of the PSD-95 organization, we recorded STED images every 20 seconds. To speed up the acquisition time, we collected single-plane images. Magnified views of an exemplary imaging series are shown in Figure 3.21. The nanoscopic resolution enabled to identify rearrangements in a fraction of PSD-95 organizations even in this short time-resolution. The major changes were observed in the case of complex-shaped PSD-95 structures that altered their perforations, while keeping their surface areas predominantly constant. However, our results revealed a morphological stability of most PSD-95 arrangements on a short-term time scale.

Even if preliminary (one mouse per time-lapse condition examined), our results indicate that PSD-95 might undergo structural changes within the synapse on a short- and long-term time scale. Further investigations are needed to ascertain whether the observed changes are of a lasting nature. Furthermore, the analysis should be extended to 3D images to better appreciate the overall spine morphology and PSD-95 rearrangements. In this respect, the development and use of brighter and more photostable dyes will also be beneficial. Notably we did not observe any kind of photodamage throughout the time-lapse recordings. 

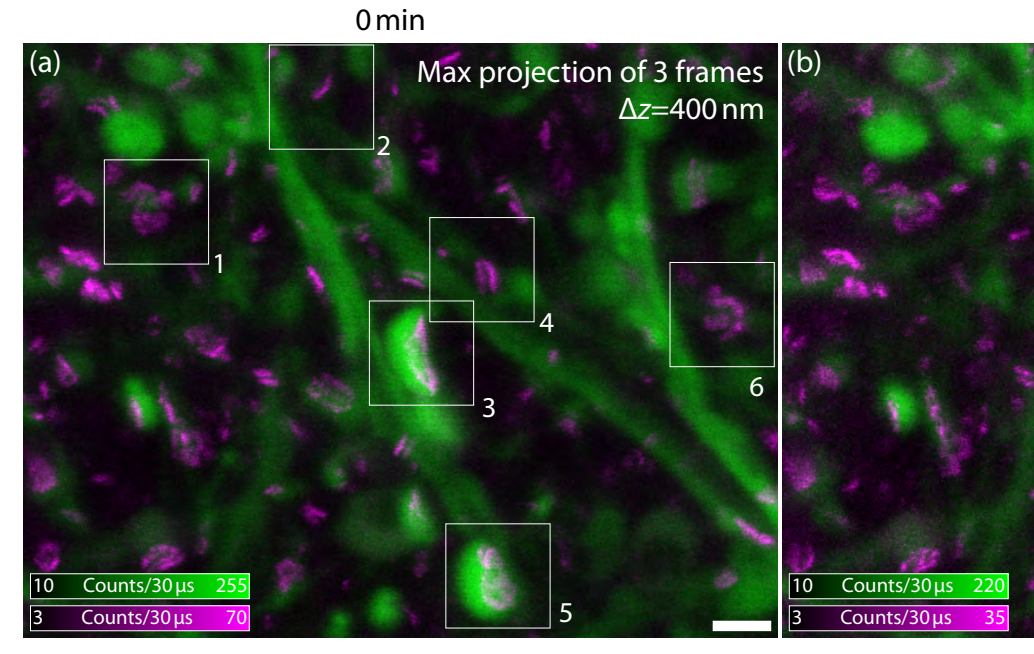

$8 \min$
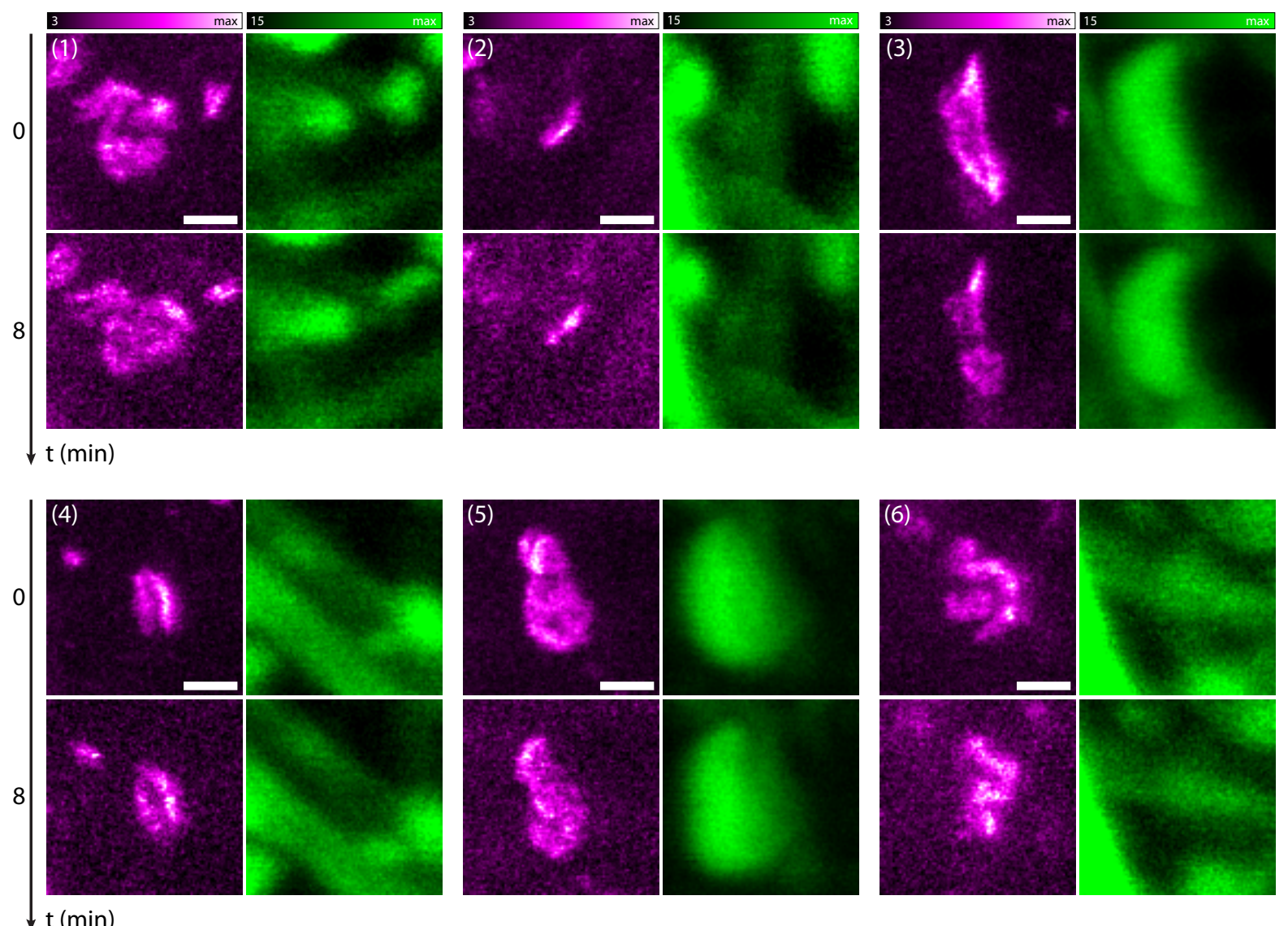

Figure 3.20: Long-term time-lapse imaging reveals rearrangements of individual PSD-95 structures in a living

\section{mouse}

Organizations of PSD-95 at given time points (a: start, 0 minutes; b: after 8 minutes). (a, b) Overlays of the STED maximum intensity projections (max projection) of PSD-95 (magenta) with the corresponding confocal max projections of cytosolic eGFP (green). Endogenous PSD-95-HaloTag was labeled with the SiR-Halo-ligand and imaged at different depths within the molecular layer of the visual cortex of a living mouse and at two different time points. The individual images were recorded as stacks with $400 \mathrm{~nm}$ axial step size (acquisition order: $z=0, z=-400 \mathrm{~nm}, z=+400 \mathrm{~nm}$, the $z$-axis corresponds to the propagation direction of the incident light), whereby the three imaging modes (STED, confocal, and confocal eGFP reference) were acquired line-wise both at each time point and $z$-plane. (1-6) Insets of the indicated regions from (a). Prior to the max projection processing, all images were corrected for drift based on the calculated sample drift of the confocal eGFP images from $z=0$, and smoothed with a one pixel wide 2D Gaussian filter. Detailed imaging parameters are listed in Table A.1. Scale bars: $1 \mu \mathrm{m}(\mathrm{a}, \mathrm{b}), 500 \mathrm{~nm}(1-6)$. 
20
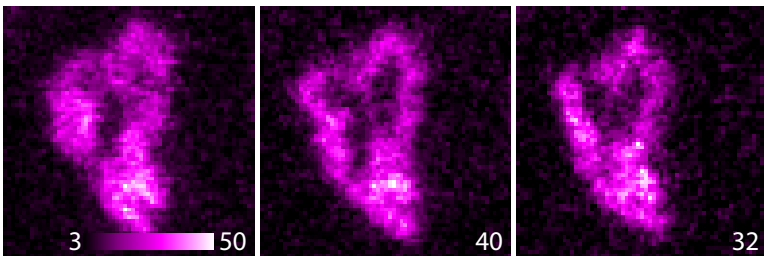

32
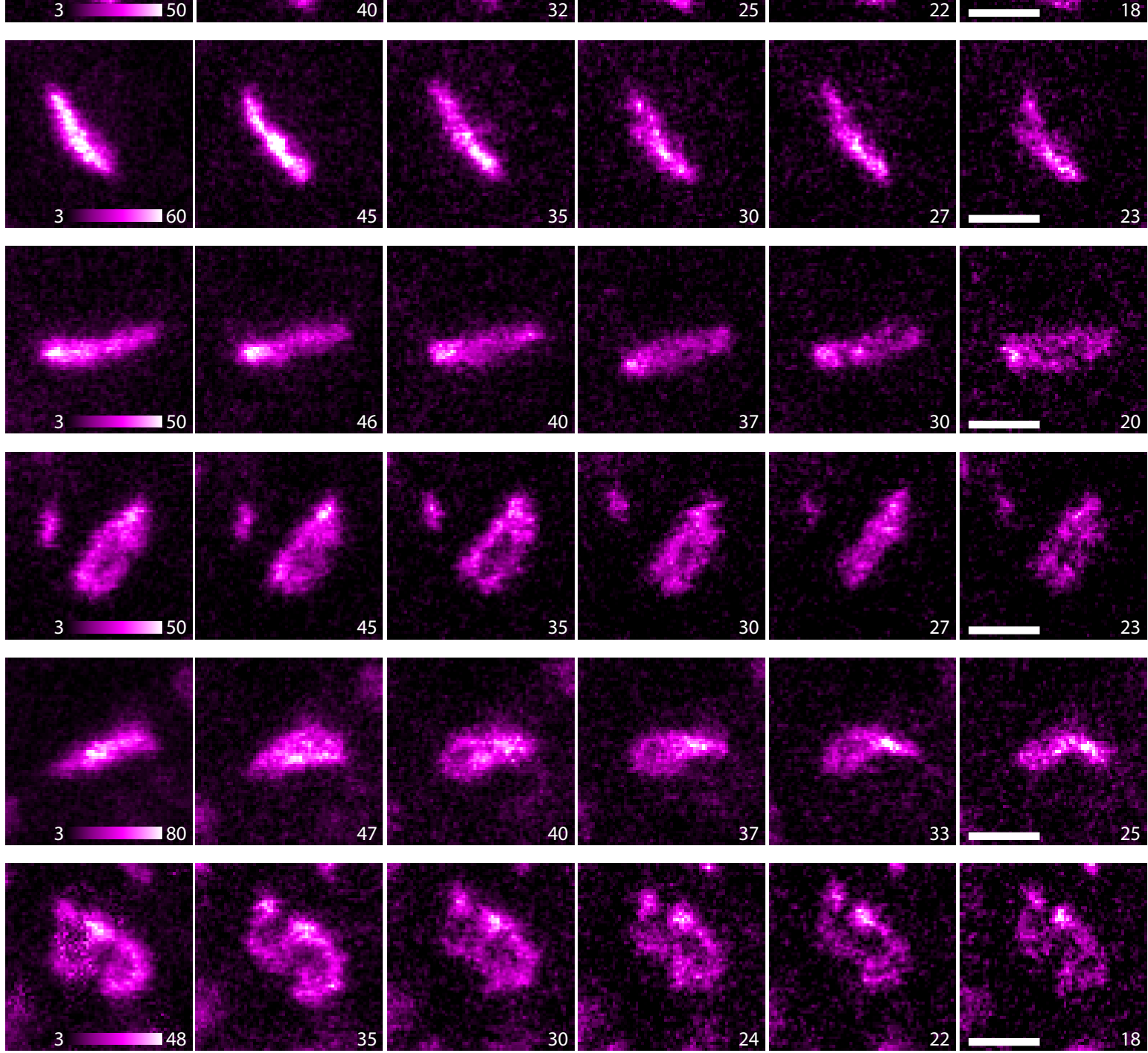

Figure 3.21: Short-term time-lapse imaging reveals rearrangements of individual PSD-95 structures in a living mouse

Several PSD-95 structures were recorded repeatedly every 20 seconds for almost two minutes. Endogenous PSD-95HaloTag was labeled with the SiR-Halo-ligand and imaged at the same focal plane within the molecular layer of the visual cortex of a living mouse. Provided values in the bottom right corners specify the maximum counts of the individual images. All images were corrected for drift and were smoothed with a one pixel wide 2D Gaussian filter. Detailed imaging parameters are listed in Table A.1. Scale bars: $500 \mathrm{~nm}$. 


\subsubsection{Summary}

The aim of this thesis was to image for the first time the distribution and substructure of a synaptic protein in vivo by STED nanoscopy. The subject of our investigation was the postsynaptic protein PSD-95, which we studied in a transgenic knock-in mouse line expressing endogenous PSD-95 in fusion with the self-labeling enzyme HaloTag. The endogenous PSD-95-HaloTag was stained with a HaloTag-ligand (610CP-Halo or SiR-Halo) using intracortical dye injection into the first layer of the visual cortex ( $35 \mu \mathrm{m}$ depth below the cortex surface) one hour prior to STED imaging.

Using this approach, we performed STED nanoscopy through a cranial window in anesthetized transgenic mice (PSD95-HaloTag) and demonstrated the benefits of STED nanoscopy for imaging PSD-95 in vivo with far-red fluorescent markers. The HaloTag-ligands provided bright, consistent labeling with low unspecific background staining and permitted the observation of structural features as small as $70-85 \mathrm{~nm}$ (FWHM), which is close to the achievable resolution of the custombuilt STED nanoscope (see Section 3.2.3). We studied the shape, size, and distribution of endogenous PSD-95 in cortical dendrites of pyramidal neurons at 5-25 $\mu \mathrm{m}$ below the brain surface, and found that the structures of PSD-95 were highly diverse and varied considerably in shape and size between synapses. We resolved their individual substructures with unprecedented resolution and found that larger structures were not simply puncta, but rather complex PSD-95 arrangements. Based on the appearance of PSD-95 organizations, we classified them into three groups: a) small round- or ovalshaped, b) elongated, and c) complex. This distinction was not possible with confocal imaging, as small round- or oval-shaped and complex structures were not distinguishable. Overall, the size of the PSD-95 arrangements spanned from an average dimension of $124 \pm 21 \mathrm{~nm}$ and $223 \pm 46 \mathrm{~nm}$ (mean \pm SD short axis and mean \pm SD long axis) in small puncta to $1077 \pm 251 \mathrm{~nm}$ and $592 \pm 210 \mathrm{~nm}$ (mean \pm SD longest side and mean \pm SD perpendicular orientated side through the center of the longest side) for the most complex structures with irregular borders and perforations.

The classification and size analysis of various nanoscale arrangements of PSD-95, however, were dependent on the orientation of the synapse with respect to the focal plane. By recording image stacks, we identified a connection between the elongated and complex-shaped PSD-95 structures. With eGFP/YFP labeling of the dendritic morphology as reference, we noted that the complex substructures were observed primarily from the en face view, while elongated substructures of PSD95 were imaged from the side-view. Notably, the STED images revealed mostly continuous organizations of PSD-95, though distinct interruptions fragmenting the morphology were sometimes present.

Benefiting from the high photostability afforded by the fluorescent labels, we also performed timelapse STED imaging of individual PSD-95 structures on short- and long-term time scales. Consecutive recordings revealed morphological rearrangements in a fraction of PSD-95 complexes in both time frames. Further investigations of the time-dependent variations in PSD-95 organizations are necessary to fully characterize these preliminary findings. Notably, we did not observe any sign of photodamage throughout the time-lapse recordings.

Altogether, the combination of in vivo STED nanoscopy, a transgenic mouse line with PSD-95, and bright and photostable far-red fluorescent HaloTag-ligands allowed us to perform the first superresolution study of an endogenously tagged synaptic protein in living mice. STED nanoscopy resolved structural diversity of PSD-95 organizations, revealing complex morphologies that are not resolved by confocal microscopy. 


\section{CONCLUSIONS AND OUTLOOK}

The architecture and function of synapses are best studied in living animals under physiological conditions where a multitude of cell types (e.g. astrocytes and oligodendrocytes) and the extracellular matrix are present and all homeostatic regulatory mechanisms are intact. Two-photon excitation microscopy provided insights into the synaptic structure in living animals even during activity; however, its spatial resolution is only capable of detecting major rearrangements of the whole compartment (Grutzendler et al., 2002; Trachtenberg et al., 2002; Gray et al., 2006; Helmchen, 2009). In the last decades, super-resolution microscopy techniques have become a powerful tool to resolve the substructure of synapses on the nanometer-scale with molecular specificity in vitro (reviewed by Sigrist and Sabatini, 2012; Maglione and Sigrist, 2013; Tønnesen and Nägerl, 2013; Willig and Barrantes, 2014). However, no super-resolution data has been published so far on the molecular organization of synapses in vivo due to the extraordinary complexity of the experiments and the lack of adequate labeling tools. In this thesis, we demonstrated for the first time the capability of STED nanoscopy to study the distribution and substructure of a synaptic protein in its native environment in a living mouse. In order to accommodate the demanding nature of these experiments, a robust STED nanoscope, stable animal preparation, and efficient fluorescent labeling were optimized for synergistic function.

We constructed a modular nanoscope with a compact STED unit $(25 \mathrm{~cm} \times 30 \mathrm{~cm})$, whose design concept best fulfilled the specific requirements for imaging the brain in living mouse with maximal optical stability. By minimizing the size of the design, we overcame the challenge of the typically limited stability of upright STED configurations. The setup was equipped with a high-NA glycerolimmersion objective lens, which enabled super-resolution imaging within the dense neuronal network of the neocortex of a living mouse. To reduce light scattering and the risk of tissue damage, we optimized the setup for the far-red spectral range with a depletion laser wavelength of $775 \mathrm{~nm}$. Two excitation lasers $(594 \mathrm{~nm}$ and $640 \mathrm{~nm})$ and two far-red detection channels $(623 \pm 12 \mathrm{~nm}$ and $685 \pm 35 \mathrm{~nm}$ ) were implemented to allow flexibility in the choice of the fluorophores and to enable two-color STED imaging. Importantly, while previous in vivo STED experiments with yellow fluorescent protein (YFP) labeling (excitation: $488 / 490 \mathrm{~nm}$, depletion: 592/590 nm) revealed swelling but no degradation of dendrites (Berning et al., 2012; Willig et al., 2014), we did not observe any signs of tissue photodamage throughout the duration of STED imaging in living mice, thereby, further encouraging the use of the far-red spectral configuration in in vivo nanoscopy.

In vivo nanoscale imaging intrinsically introduces new challenges, not present in two-photon excitation microscopy due to its lower operating resolution. Indeed, motion artifacts induced by the movement of the mouse in in vivo STED nanoscopy have been previously shown to compromise the quality of obtained images (Willig et al., 2014). The motions stem primarily from the heartbeat and breathing, which we limited by anesthetizing the animal and performing artificial ventilation, as previously described (Holtmaat et al., 2009; Berning et al., 2012; Willig et al., 2014). Moreover, we improved the surgical steps involved, with particular emphasis on the mechanical stability of the mouse head fixation. We designed a head holder with larger surface contact area and discovered a new and better bonding compound than the one previously used. Both improvements extended the mechanical stability and significantly reduced the motion artifacts visible in nanoscopic images. We saw artifacts only in close proximity of the pulsating arteries. The established mouse preparation routine is thus highly effective and can be implemented as a reference protocol for in vivo imaging of the cortex. 
In addition to suitable STED instrumentation and stable animal preparation, we developed two labeling strategies for in vivo STED imaging based on far-red fluorescent proteins (FPs) or organic fluorescent dyes. One approach relied on the overexpression of FPs genetically fused to the protein of interest, whereas the other utilized the self-labeling enzyme HaloTag as a fusion partner, which was then covalently tagged by a membrane-permeable fluorescent probe. With these tools at our disposal, we demonstrated for the first time the feasibility of performing in vivo STED imaging using far-red FPs and organic dyes in combination with a $775 \mathrm{~nm}$ depletion laser. We identified the two FPs, mNeptune2 and mCardinal, as well as the two dyes, 610CP-Halo and SiR-Halo, as the most suitable fluorophores. In terms of brightness and photostability, both organic dyes exhibited a far superior performance compared to the FPs and were therefore used for labeling synaptic proteins in living mice. The flexibility and wide spectrum of available substrates make the use of self-labeling enzymes attractive for two-photon excitation imaging as well. This tool could also be conceivably used to study protein turnover in vivo by simple injection of spectrally separated dyes at different time points.

To study a synaptic protein in its native environment with super-resolution, we used the transgenic mouse line PSD95-HaloTag, which was engineered to express endogenous postsynaptic density 95 (PSD-95) protein in fusion with the self-labeling enzyme HaloTag. Compared to virus-mediated protein expression, the advantages of this mouse line are: (i) no overexpression ${ }^{43}$ of the fusionconstruct (and artifact thereof), (ii) no alterations in the localization and functionality of the protein of interest, and (iii) flexibility in the choice of the fluorescent HaloTag-probe to be used (and the corresponding flexibility in the wavelength range used, in contrast to transgenic mice with a GFP- or YFP-tagged protein of interest where only certain wavelengths can be used). By injecting 610CP-or SiR-Halo into the first layer of the visual cortex, STED nanoscopy resolved individual structures of endogenous PSD-95 with unprecedented lateral resolution and found that PSD-95 arrangements were highly diverse and varied considerably in shape and size between synapses. Larger structures were not simply puncta (as seen with confocal microscopy), but rather complex PSD-95 arrangements with irregular borders and perforations never reported before in such detail in optical microscopy studies. Moreover, with respect to the reference labeling, our results indicate that the complex-shaped organizations of PSD-95 tend to occur in the largest spine heads. This seems to be consistent with previous EM studies, which have also associated the existence of larger PSD-95 complexes with increased synaptic activity (Harris and Stevens, 1989; Geinisman et al., 1993; Stewart et al., 2005).

Based on the appearance of PSD-95 organizations in the 2D images, we classified them into three groups (i.e. small round- or oval-shaped, elongated, and complex) and analyzed their sizes. The smallest structural features were 70-85 nm (FWHM) in width, which corresponds to an at least 4-fold lateral resolution improvement of our STED nanoscope over diffraction-limited optical microscopy. While the custom-built STED nanoscope achieved a lateral resolution of $52 \mathrm{~nm}$ on fluorescent beads with the maximum available depletion laser power, the resolution of the in vivo experiments was primarily limited by photobleaching precluding the use of comparably high laser powers. Nevertheless, the attained at least 4-fold lateral resolution improvement matches those reported in earlier STED imaging studies on cultured brain slices or in living mice (Berning, 2011; Urban et al., 2011; Willig et al., 2014).

\footnotetext{
${ }^{43}$ Overexpression of PSD-95 can increase synaptic strength (Stein et al., 2003; Ehrlich and Malinow, 2004) and dendritic spine size (El-Husseini et al., 2000).
} 
Overall, the size of the PSD-95 arrangements spanned from an average dimension of $124 \mathrm{~nm} \times 223 \mathrm{~nm}$ in the case of small puncta to $592 \mathrm{~nm} \times 1077 \mathrm{~nm}$ for the most complex structures. Notably, we found mostly continuous organizations of PSD-95 in elongated and complex structures in the STED images, though distinct interruptions sometimes fragmented the structural morphologies observed. The high diversity and complexity of PSD-95 arrangements are in agreement with recent super-resolution studies on the organization of PSD-95 in fixed neurons or fixed brain slices (Dzyubenko et al., 2016; Broadhead et al, 2016). STED nanoscopy of synapses in the hippocampus of fixed brain slices revealed that endogenous PSD-95-eGFP is organized into nanoclusters with a single oval nanocluster size of $90 \mathrm{~nm} \times 200 \mathrm{~nm}$ (Broadhead et al, 2016). There, in $65 \%$ of the synapses, PSD-95 consisted of a single nanocluster, whereas some contained multiple nanoclusters that were separated by a mean distance of 100-300 nm. Some multi-nanoclusters were resolved in a ring-like formation (Broadhead et al, 2016). Our in vivo results also identified small, elliptical PSD-95 organizations with a size comparable to the finding from Broadhead et al, 2016. In contrast, the complex-shaped PSD-95 structures in our data were not organized into nanoclusters but instead clearly showed ring-like mostly continuous formations with perforations, which seem to be more consistent with earlier EM studies (Carlin et al., 1980; Nieto-Sampedro et al., 1982; Geinisman, 1993). Moreover, we identified that the smallest features of these complex structures were $107 \pm 28 \mathrm{~nm}$ in width, which might be a hint that small round- or oval-shaped structures with almost the same size share a common smallest unit with the complex-shaped organizations. However, neither the complex nor the elongated PSD-95 structures exhibited a distinct clustered nano-organization. This difference with previous reports might be ascribed to the optical resolution that may not have been sufficient to detect single nanoclusters. The smallest observed PSD-95 features in our study measured a size of $70-85 \mathrm{~nm}$, which is approximately equal to the determined lateral resolution capability $(90 \mathrm{~nm}$ FWHM) of our STED nanoscope at the utilized power (see Section 3.2.3). It seems possible that these features are much smaller than those identified in our experiments. However, as the resolution of our STED nanoscope was nearly the same as the resolution of $85 \mathrm{~nm}$ in Broadhead et al, 2016, we should be able to identify nanoclusters of a size of 90-200 nm, separated 100-300 nm apart. A potential explanation is that the reported nanoclusters may be artifacts caused by the fixation process. So far, the effects of different sample fixations on the image structure in super-resolution microscopy have not been explored in detail, but some studies have already reported image artifacts due to conventional fixation protocols (Whelan and Bell, 2015; Bachmann et al., 2016). It might also be that the described nanoclusters in Broadhead et al, 2016 are an effect of the image processing with a Gaussian kernel filter of $40 \mathrm{~nm}$ size, followed by a threshold to subtract the background. Further studies on fixed brain sections from the PSD95-HaloTag mouse will clarify whether the discrepant results are an artifact induced by the fixation or due to the limited resolution used in the in vivo studies owing to photobleaching.

We classified the PSD-95 structures based on their appearance in 2D images with a low resolution along the optical axis, which then resemble add up projections of the native 3D structure onto the 2D image plane. We speculate that the appearance of PSD-95 arrangements is dependent on the orientation of the synapse with respect to the focal plane. Indeed, we observed elongated structures mostly arranged along the postsynaptic membranes of dendritic spines in side-views of synapses (i.e. in case where the reference labeling (Lifeact-YFP or cytoplasmic eGFP) showed the dendritic spine head connected via spine neck with the dendritic shaft). In contrast, complex-shaped organizations of PSD-95 were typically identified in en face views of synapses (reference labeling showed elliptical disks). Our recorded image stacks revealed that PSD-95 arrangements in one focal plane would be classified with elongated shapes, but actually exhibited complex and perforated 
structures in a parallel imaging plane located $400 \mathrm{~nm}$ apart along the $z$-axis, suggesting that a connection between elongated and complex-shaped structures does indeed exist. This finding is in agreement with another STED imaging study (Dzyubenko et al., 2016) that also resolved PSD-95 as bar-shaped or ring-like organizations.

In the last part of the results, we showed preliminary data regarding the dynamics of PSD-95. Previously, a study on dynamics of overexpressed PSD-95 in vivo was reported by Gray et al., 2006 using two-photon excitation microscopy. They showed that a subset of PSD-95-GFP or -paGFP organizations were stable over days, while single PSD-95 molecules were rapidly redistributed between neighboring spines. Based on our short- and long-term time-lapse imaging ( 2 and $~ 10$ min, respectively), we observed that PSD-95 might be structurally rearranged within the synapse. While the small round PSD-95 arrangements were rather stable, the complex shapes revealed a rearrangement of their perforations. In the analysis of our data, however, several points should be taken into consideration: (i) photobleaching might introduce imaging artifacts, e.g. structural changes become visible due to partial bleaching, (ii) focus drift along the optical axis and (iii) rotation or dynamics of spine morphology causing a different appearance of the structure. In our measurements, we cannot completely rule out that any of the points mentioned above might have had a visible effect on our images. As to the first concern, lower depletion laser power would reduce photobleaching; however, it would also result in lower resolution which may not be sufficient to resolve the complex PSD-95 arrangements. Concerns (ii) and (iii) could be better mitigated by using 3D imaging to appreciate the overall spine morphology and PSD-95 (re-)arrangements. Further investigations are needed to ascertain whether the observed changes of PSD-95 are of a lasting nature and whether they are correlated to an overall reshaping of the spine head. These experiments are possible only in vivo, since the most complex PSD-95 arrangements have not been reported in such a detail in cell- or tissue culture experiments.

\section{Future directions}

Although our presented data provide a representative view of the PSD-95 nanostructures in vivo, higher spatial resolution will be necessary to further resolve the smallest structural units of PSD-95. In our experiments, the resolution was mostly limited by photobleaching of the fluorescent marker. However, due to their exceptional brightness and photostability, organic fluorophores are still favored for future experiments. Within this thesis, we focused on the most widely-used dye SiR as well as on promising dyes developed in our department that match the specifications of our custombuilt STED nanoscope in terms of excitation/depletion wavelengths and detection channels. In addition to the tested fluorescent probes, other HaloTag-dye candidates such as Janelia Fluor 585 or 646 (Grimm et al., 2015; Grimm et al., 2017; both commercially available from Tocris Bioscience, Bristol, United Kingdom), ATTO590 (Bottanelli et al., 2016; commercially available from ATTO-TEC, Siegen, Germany), or SiR700 (an even more red-shifted version of SiR, Lukinavičius et al., 2016) could be investigated in future in vivo experiments. Moreover, new fluorescent dyes are being continually designed, and these developments may prove to be particularly useful in future experiments. As our approach with a transgenic mouse benefits from the flexibility of the self-labeling HaloTag technology, we are able to rapidly investigate newly designed cell-permeable dyes and test whether they are more suitable than the ones used so far. However, new promising dyes may require a modification of the optical setup to meet their spectral properties (tuning of the depletion laser wavelength, implementing other excitation laser wavelengths, and change of optical components e.g. filters). 
In addition to the development of brighter and more photostable dyes, recent advancements in STED nanoscopy (see Section 1.3) could be adapted to our approach to reduce photobleaching. A simple but efficient method would be to implement relatively long depletion laser pulses $(600-$ 1000 ps pulse duration), which are less harmful due to lower peak intensities (Dyba and Hell, 2003; Göttfert et al., 2013; Castello et al., 2016). Commercially available high power laser sources with long pulses (approx. 1000 ps) typically have a lower repetition rate $(20-40 \mathrm{MHz})$, which in turn increases the acquisition time. In live-cell applications, however, fast imaging speed is desired to avoid artifacts due to sample dynamics or drift. To counteract this, the imaging speed could also be further improved by parallelization of the scanning with multiple beams (Bingen et al., 2011; Bergermann et al., 2015). Another approach to reduce photobleaching would be the implementation of MINFIELDSTED, which significantly reduces light irradiation of the sample by confining the field-of-view to the minimum of the depletion pattern (Göttfert et al., 2017). Since a higher depletion laser intensity can then be applied, it enables imaging at higher spatial resolution with enhanced image contrast. However, due to its design, this new method is limited to very small imaging fields of for example $100 \mathrm{~nm} \times 100 \mathrm{~nm}$. Although our results showed that the smallest features of the complex PSD-95 structures were $107 \mathrm{~nm}$ in width, which borders the limits of MINFIELD scanning area, MINFIELD may be used to unravel the PSD-95 arrangements with an expected lateral resolution down to $20 \mathrm{~nm}$. More recently, an approach termed DyMIN was reported, which considerably reduces the overall light irradiation of the sample by applying the maximum depletion laser power only if a significant amount of structure is present (Heine et al., 2017). In this way, DyMIN enables imaging at higher spatial resolution ( $25 \mathrm{~nm}$ ) with enhanced image contrast and, at the same time, reduces bleaching. Imaging of synaptic sites may benefit immensely from DyMIN, since most of the pixels in the image usually do not contain any information and the structures are confined in space. This has already been demonstrated on cultured cells (Heine et al., 2017). Therefore, we can anticipate that in vivo imaging with DyMIN will improve the resolution, though at the expense of longer acquisition times. On a longer perspective, intravital implementation of MINFLUX, which achieves true molecular resolution (see Section 1.2; Balzarotti et al., 2017), can be speculated. So far, however, this novel super-resolution approach has only been demonstrated in proof-of-principle studies. Its application to observe the structure, distribution, and dynamics of proteins in the living mouse brain would still require major developments, with particular concern to the size of the field-of-view, which is currently limited to $\sim 100 \mathrm{~nm} \times 100 \mathrm{~nm}$.

As presented in this thesis, PSD-95 scaffolds have a highly three-dimensional geometry; threedimensional super-resolution would thus be even more beneficial. So far, we have only used the nanoscope for super-resolution capability in lateral dimensions because the phase retardation pattern for 3D is more sensitive to aberrations, which is particularly challenging when imaging thick specimens (Booth et al., 2015; Patton et al., 2016). The custom-built STED nanoscope is already equipped with a freely programmable spatial light modulator and, thus, the required phase mask for axial resolution enhancement, the so-called bottle-beam or $z$-doughnut (Klar et al., 2000; Harke et al., 2008; Wildanger et al., 2009; Osseforth et al., 2014), could be additionally applied to the depletion beam. However, this would require a relatively high depletion laser power for the simultaneous implementation of two phase masks to provide enough depletion intensity for the saturation of the fluorophores in all three dimensions. Typically, 3D STED imaging with a significantly enhanced axial resolution is enabled at the expense of both some lateral resolution and photobleaching. Therefore, finding the best tradeoff for 3D STED nanoscopy requires an accurate optimization of all imaging parameters. 
Regarding the biological aspects of this work, future in vivo experiments could aim at studying the distribution, localization, and interaction of two or more proteins (e.g. PSD-95 and transmembrane AMPA receptor regulating proteins like stargazin (Deng et al., 2006)). The custom-built STED nanoscope presented here is already capable of performing two-color STED measurements, which require a pair of fluorophores that match the specification of the setup. In the scope of this thesis, we already tested the dye $580 \mathrm{CP}$, which may be used together with the dye SiR for future two-color experiments. However, the less red-shifted fluorophore (here 580CP) has a smaller cross-section for stimulated emission, and a higher depletion laser intensity is necessary to achieve the same resolution in both detection channels, conversely resulting in higher photobleaching of the more red-shifted fluorophore (here SiR). A modulation of the depletion laser intensity during image acquisition would be beneficial to be able to perform two-color STED imaging simultaneously with optimal resolutions in both color channels (Sidenstein et al., 2016). This concept, called multilevel STED, would require the employment of fast acousto- or electro-optic modulators, a modification of the electronics, and adjustments of the acquisition software.

If long-term imaging over days or weeks is favored, the established surgical preparation within this thesis is not appropriate due to its highly invasive nature (artificial ventilation via tracheostomy and continuous anesthesia through the Vena jugularis). By changing the method of anesthesia (e.g. isoflurane inhalation), chronic experiments would be possible in principle. The feasibility of longterm studies has already demonstrated with two-photon excitation microscopy via the use of a cranial window to investigate structural plasticity over a period of weeks (Grutzendler et al., 2002; Trachtenberg et al., 2002; Gray et al., 2006; Holtmaat et al., 2009). One of the major limitations for long-term STED imaging, though, may be the labeling. Usually, transgenic mouse lines expressing the protein of interest in fusion with an FP or virus-mediated expression of fusion constructs are used in chronic experiments. FPs are less photostable and bright, and therefore the employment of genetically expressed self-labeling tags would be preferable. The transgenic mouse line PSD95HaloTag benefits from the flexibility of the self-labeling technology and could be utilized for longterm experiments. The only disadvantage, however, would be the need to apply the fluorescent probe before every imaging session. The current labeling approach of intracortical injections restricts multiple dye applications at different time points. Nevertheless, this can be overcome via injection of a blood-brain barrier-permeable ligand into the tail vein as recently reported by Grimm et al., 2017.

In summary, the combination of a robust in vivo STED nanoscope, a transgenic mouse line with PSD95-HaloTag, and bright and photostable far-red fluorescent HaloTag-ligands allowed us to perform the first super-resolution study of an endogenously tagged synaptic protein in the brains of living mice. STED nanoscopy resolved structural diversity of PSD-95 organizations with unprecedented clarity, revealing complex morphologies unresolved by confocal microscopy or by super-resolution imaging in cell- or tissue culture experiments. Our results highlight the presence of differences between the organization of postsynaptic sites in vitro and in vivo, and therefore the necessity to scrutinize these nanoclusters in greater detail. The results presented here indicate the importance of in vivo super-resolution microscopy and introduce a novel approach for future investigations of the molecular organization and function of the brain in living animals. 


\section{BIBLIOGRAPHY}

Abbe, E. (1873). Beiträge zur Theorie des Mikroskops und der mikroskopischen Wahrnehmung. Arch Mikrosk Anat, 9:413-420.

Arthur, C. P., et al. (2007). Electron tomographic methods for studying the chemical synapse. Methods Cell Biol, 79 : 241-257.

Auksorius, E., et al. (2008). Stimulated emission depletion microscopy with a supercontinuum source and fluorescence lifetime imaging. Opt Lett, 33(2): 113-115.

Azevedo, F. A. C., et al. (2009). Equal numbers of neuronal and nonneuronal cells make the human brain an isometrically scaled-up primate brain. J Comp Neurol, 513(5): 532-541.

Bachmann, M., Fiederling, F. and Bastmeyer, M. (2016). Practical limitations of superresolution imaging due to conventional sample preparation revealed by a direct comparison of CLSM, SIM and dSTORM. J MicrosC, 262(3): 306-315.

Balzarotti, F., et al. (2017). Nanometer resolution imaging and tracking of fluorescent molecules with minimal photon fluxes. Science, 335(6325): 606-612.

Bayer, A. (1871). Über eine neue Klasse von Farbstoffen. Ber Dtsch Chem Ges, 4: 555-558.

Bayés, A., et al. (2011). Characterization of the proteome, diseases and evolution of the human postsynaptic density. Nat Neurosci, 14(1): 19-21.

Béïque, J.-C. and Andrade, R. (2003). PSD-95 regulates synaptic transmission and plasticity in rat cerebral cortex. $\underline{J}$ Physiol, 546(Pt3): 859-867.

Bergermann, F., Alber, L., Sahl, S. J., Engelhardt, J. and Hell, S. W. (2015). 2000-fold parallelized dual-color STED fluorescence nanoscopy. Opt Express, 23(1): 211-223.

Berning, S. (2011). STED Nanoscopy of the Living Brain. Dissertation, University of Göttingen.

Berning, S., Willig, K. I., Steffens, H., Dibaj, P. and Hell, S. W. (2012). Nanoscopy in a Living Mouse Brain. Science, 335(6068): 551.

Bethge, P., Chéreau, R., Avignone, E., Marsicano, G. and Nägerl, U. V. (2013). Two-photon excitation STED microscopy in two colors in acute brain slices. Biophys J, 104(4): 778-785.

Betzig, E. (1995). Proposed method for molecular optical imaging. Opt Lett, 20(3): 237-239.

Betzig, E., et al. (2006). Imaging intracellular fluorescent proteins at nanometer resolution. Science, 313(5793): $1642-1645$.

Bingen, P., Reuss, M., Engelhardt, J. and Hell, S. W. (2011). Parallelized STED fluorescence nanoscopy. Opt Express, 19(24): 23716-23726.

Booth, M. J. (2014). Adaptive optical microscopy: the ongoing quest for a perfect image. Light: Sci and Appl, 3 : e165.

Booth, M., Andrade, D., Burke, D., Patton, B. and Zurauskas, M. (2015). Aberrations and adaptive optics in superresolution microscopy. J Microsc-Oxford, 64(4): 251-261.

Borgdorff, A. J. and Choquet, D. (2002). Regulation of AMPA receptor lateral movements. Nature, 417(6889): 649-653.

Born, M. and Wolf, E. (1999). Principles of Optics, 7th edition. Cambridge University Press, Cambridge, United Kingdom.

Bossi, M., Fölling, J., Dyba, M., Westphal, V. and Hell, S. W. (2006). Breaking the diffraction resolution barrier in farfield microscopy by molecular optical bistability. New J Phys, 8(11): 275.

Bottanelli, F., et al. (2016). Two-colour live-cell nanoscale imaging of intracellular targets. Nat Commun, 7: 10778. 
Broadhead, M. J., et al. (2016). PSD95 nanoclusters are postsynaptic building blocks in hippocampus circuits. $\underline{\text { Sci }}$ Rep, 6: 24626.

Bückers, J., Wildanger, D., Vicidomini, G., Kastrup, L. and Hell, S. W. (2011). Simultaneous multi-lifetime multicolor STED imaging for colocalization analyses. Opt Express, 19(4): 3130-3143.

Butkevich, A. N., et al. (2016). Fluorescent Rhodamines and Fluorogenic Carbopyronines for Super-Resolution STED Microscopy in Living Cells. Angew Chem Int Ed, 55(10): 3290-3294.

Butkevich, A. N., et al. (2017). Hydroxylated Fluorescent Dyes for Live-Cell Labeling: Synthesis, Spectra and SuperResolution STED Microscopy. Chem Eur J, 23(50): 12114-12119.

Butkevich, A. N., Lukinavičius, G., D'Este, E. and Hell, S. W. (2017). Cell-Permeant Large Stokes Shift Dyes for Transfection-Free Multicolor Nanoscopy. J Am Chem Soc, 139(36): 12378-12381.

Carlin, R. K., Grab, D. J., Cohen, R. S. and Siekevitz, P. (1980). Isolation and characterization of postsynaptic densities from various brain regions: enrichment of different types of postsynaptic densities. J Cell Biol, 86(3): 831-845.

Castello, M., et al. (2016). Gated-sted microscopy with subnanosecond pulsed fiber laser for reducing photobleaching. Microsc Res Techniq, 79(9): 785-791.

Cetin, A., Komai, S., Eliava, M., Seeburg, P. H. and Osten, P. (2006). Stereotaxic gene delivery in the rodent brain. Nat Protoc, 1(6): 3166-3173.

Chalfie, M., Tu, Y., Euskirchen, G., Ward, W. W. and Prasher, D. C. (1994). Green fluorescent protein as a marker for gene-expression. Science, 263(5148): 802-805.

Chen, L., et al. (2000). Stargazin regulates synaptic targeting of AMPA receptors by two distinct mechanisms. Nature, 408(6815): 936-943.

Chen, X., et al. (2005). Mass of the postsynaptic density and enumeration of three key molecules. $\underline{P \text { Natl Acad Sci }}$ USA, 102(32): 11551-11556.

Chen, X., Winters, C. A. and Reese, T. S. (2008). Life inside a thin section: tomography. J Neurosci, 28(38): 9321-9327.

Cheng, D., et al. (2006). Relative and absolute quantification of postsynaptic density proteome isolated from rat forebrain and cerebellum. Mol Cell Proteomics, 5(6): 1158-1170.

Cho, K. O., Hunt, C. A. and Kennedy, M. B. (1992). The rat brain postsynaptic density fraction contains a homolog of the Drosophila discs-large tumor suppressor protein. Neuron, 9(5): 929-942.

Choquet, D. (2010). Fast AMPAR trafficking for a high-frequency synaptic transmission. Eur J Neurosci, 32(2): $250-260$.

Chu, J., et al. (2014). Non-invasive intravital imaging of cellular differentiation with a bright red-excitable fluorescent protein. Nat Methods, 11(5): 572-578.

Chung, W.-S., Welsh, C. A., Barres, B. A. and Stevens, B. (2015). Do glia drive synaptic and cognitive impairment in disease? Nat Neurosci, 18(11): 1539-1545.

Chung, W.-S., Allen, N. J. and Eroglu, C. (2015). Astrocytes control synapse formation, function, and elimination. Cold Spring Harb Perspect Biol, 7(9): a020370.

Clay, R. S. and Court, T. H. (1933). The History of the Microscope: Compiled from Original Instruments and Documents, up to the Introduction of the Achromatic Microscope. Nature, 131:219-221.

Coons, A., Creech, H., Jones, R. N. and Berliner, E. (1942). The demonstration of pneumococcal antigen in tissues by the use of fluorescent antibody. J Immunol, 45(3): 159-170.

Cox, G. and Sheppard, C. J. R. (2004). Practical limits of resolution in confocal and non-linear microscopy. Microsc Res Techniq, 63(1): 18-22. 
Cranfill, P. J., et al. (2016). Quantitative assessment of fluorescent proteins. Nat Methods, 13(7): 557-562.

Cremer, C. and Cremer, T. (1978). Considerations on a laser-scanning-microscope with high resolution and depth of field. Microsc Acta, 81 (1): 31-44.

Curdt, F., et al. (2015). isoSTED nanoscopy with intrinsic beam alignment. Opt Express, 23(24): 30891-30903.

D'Este, E., Kamin, D., Gottfert, F., El-Hady, A. and Hell, S. W. (2015). STED nanoscopy reveals the ubiquity of subcortical cytoskeleton periodicity in living neurons. Cell Rep, 10(8): 1246-1251.

Dani, A., Huang, B., Bergan, J., Dulac, C. and Zhuang, X. (2010). Superresolution imaging of chemical synapses in the brain. Neuron, 68(5): 843-856.

Danzl, J. G., et al. (2016). Coordinate-targeted fluorescence nanoscopy with multiple off states. Nat Photonics, 10(2): 122-128.

Day, R. N. and Davidson, M. W. (2009). The fluorescent protein palette: tools for cellular imaging. Chem Soc Rev, 38(10): 2887-2921.

Deng, F., Price, M. G., Davis, C. F., Mori, M. and Burgess, D. L. (2006). Stargazin and other transmembrane AMPA receptor regulating proteins interact with synaptic scaffolding protein MAGI-2 in brain. J Neurosci, 26(30): 7875-7884.

Denk, W., Strickler, J. and Webb, W. (1990). Two-photon laser scanning fluorescence microscopy. Science, 248(4951): 73-76.

Donnert, G., et al. (2006). Macromolecular-scale resolution in biological fluorescence microscopy. P Natl Acad Sci USA, 103(31): 11440-11445.

Dotti, C. G., Sullivan, C. A. and Banker, G. A. (1988). The establishment of polarity by hippocampal neurons in culture. J Neurosci, 8(4): 1454-1468.

Dyba, M. and Hell, S. W. (2003). Photostability of a fluorescent marker under pulsed excited-state depletion through stimulated emission. Appl Opt, 42(25): 5123-5129.

Dzyubenko, E., Rozenberg, A., Hermann, D. M. and Faissner, A. (2016). Colocalization of synapse marker proteins evaluated by STED-microscopy reveals patterns of neuronal synapse distribution in vitro. J Neurosci Meth, 273: 149-159.

Eggeling, C., Willig, K. I., Sahl, S. J. and Hell, S. W. (2015). Lens-based fluorescence nanoscopy. Q Rev Biophys, 48(2): 178-243.

Ehrenberg, M. (2008). The green fluorescent protein: discovery, expression and development. Scientific Background on the Nobel Prize in Chemistry.

Ehrengruber, M. U., et al. (2001). Gene transfer into neurons from hippocampal slices: comparison of recombinant Semliki Forest Virus, adenovirus, adeno-associated virus, lentivirus, and measles virus. Mol Cell Neurosci, 17(5): 855-871.

Ehrlich, I. and Malinow, R. (2004). Postsynaptic density 95 controls AMPA receptor incorporation during long-term potentiation and experience-driven synaptic plasticity. J Neurosci, 24(4): 916-927.

Einstein, A. (1917). Zur Quantentheorie der Strahlung. Phys Z, 18: 121-128.

El-Husseini, A. E., Schnell, E., Chetkovich, D. M., Nicoll, R. A. and Bredt, D. S. (2000). PSD-95 involvement in maturation of excitatory synapses. Science, 290(5495): 1364-1368.

Engelhardt, J. (2010). Method and device for dynamically shifting a light beam relative to an optic which focuses the light beam. Patent application WO2010069987 A1.

Erdmann, R. S., et al. (2014). Super-resolution imaging of the Golgi in live cells with a bioorthogonal ceramide probe. Angew Chem Int Ed, 53(38): 10242-10246. 
Fernandez-Suarez, M. and Ting, A. Y. (2008). Fluorescent probes for super-resolution imaging in living cells. Nat Rev Mol Cell Biol, 9(12): 929-943.

Fölling, J., et al. (2008). Fluorescence nanoscopy by ground-state depletion and single-molecule return. Nat Methods, 5(11): 943-945.

Fornasiero, E. F. and Opazo, F. (2015). Super-resolution imaging for cell biologists. BioEssays, 37(4): 436-451.

Frank, R. A. and Grant, S. G. (2017). Supramolecular organization of NMDA receptors and the postsynaptic density. Curr Opin Neurobiol, 45: 139-147.

Frohn, J. T., Knapp, H. F. and Stemmer, A. (2000). True optical resolution beyond the Rayleigh limit achieved by standing wave illumination. P Natl Acad Sci USA, 97(13): 7232-7236.

Gautier, A., et al. (2008). An engineered protein tag for multiprotein labeling in living cells. Chem Biol, 15(2): 128-136.

Geinisman, Y. (1993). Perforated axospinous synapses with multiple, completely partitioned transmission zones probable structural intermediates in synaptic plasticity. Hippocampus 3(4): 417-434.

Geinisman, Y., et al. (1993). Structural synaptic correlate of long- term potentiation: formation of axospinous synapses with multiple, completely partitioned transmission zones. Hippocampus, 3(4): 435-445.

Giannone, G., et al. (2010). Dynamic superresolution imaging of endogenous proteins on living cells at ultra-high density. Biophys J, 99(4): 1303-1310.

Giepmans, B. N., Adams, S. R., Ellisman, M. H. and Tsien, R. Y. (2006). The fluorescent toolbox for assessing protein location and function. Science, 312(5771): 217-224.

Göppert-Mayer, M. (1931). Über Elementarakte mit zwei Quantensprüngen. Ann Phys, 401 (3): 273-294.

Görlitz, F., et al. (2014). A STED Microscope Designed for Routine Biomedical Applications. Prog Electromagn Res, 147: 57-68.

Göttfert, F., et al. (2013). Coaligned dual-channel STED nanoscopy and molecular diffusion analysis at $20 \mathrm{~nm}$ resolution. Biophys J, 105(1): L01-L03.

Göttfert, F., et al. (2017). Strong signal increase in STED fluorescence microscopy by imaging regions of subdiffraction extent. P Natl Acad Sci USA, 114(9): 2125-2130.

Gould, T. J., Burke, D., Bewersdorf, J. and Booth, M. J. (2012). Adaptive optics enables 3D STED microscopy in aberrating specimens. Opt Express, 20(19): 20998-21009.

Gray, E. G. (1959). Axo-somatic and axo-dendritic synapses of the cerebral cortex: an electron microscope study. J Anat, 93(Pt 4): 420-433.

Gray, N. W., Weimer, R. M., Bureau, I. and Svoboda, K. (2006). Rapid redistribution of synaptic PSD-95 in the neocortex in vivo. PLoS Biol, 4(11): e370.

Grimm, J. B., et al. (2015). A general method to improve fluorophores for live-cell and single-molecule microscopy. Nat Methods, 12(3): 244-250.

Grimm, J. B., et al. (2017). A general method to fine-tune fluorophores for live-cell and in vivo imaging. bioRxiv.

Grutzendler, J., Kasthuri, N. and Gan, W.-B. (2002). Long-term dendritic spine stability in the adult cortex. Nature, 420(6917): 812-816.

Gulley, R. L. and Reese, T. S. (1981). Cytoskeletal organization at the postsynaptic complex. J Cell Biol, 91(1): 298-302.

Gustafsson, M. G. L., Agard, D. A. and Sedat, J. W. (1995). Sevenfold improvement of axial resolution in 3D widefield microscopy using two objective lenses. Proc SPIE, 2412: 147-156. 
Gustafsson, M. G. L., Agard, D. A. and Sedat, J. W. (1996). 3D widefield microscopy with two objective lenses: experimental verification of improved axial resolution. Proc SPIE, 2655: 62-66.

Gustafsson, M. G. L. (2000). Surpassing the lateral resolution limit by a factor of two using structured illumination microscopy. J Microsc, 198(Pt 2): 82-87.

Gustafsson, M. G. L. (2005). Nonlinear structured-illumination microscopy: wide-field fluorescence imaging with theoretically unlimited resolution. P Natl Acad Sci USA, 102(37): 13081-13086.

Gustafsson, M. G. L., et al. (2008). Three-dimensional resolution doubling in wide-field fluorescence microscopy by structured illumination. Biophys J, 94(12): 4957-4970.

Hamamatsu Photonics (2014). LCOS-SLM: Software operation manual. Hamamatsu City, Japan: 1-38.

Hamamatsu Photonics (2014). LCOS-SLM: X10468 Series operation manual. Hamamatsu City, Japan: 1-29.

Hamamatsu Photonics (2014). "Opto-semiconductor Handbook - Chapter12: Phase spatial light modulator LCOS-SLM." 22.06.2017, from https://www.hamamatsu.com/resources/pdf/ssd/e12_handbook_Icos_slm.pdf.

Hamers-Casterman, C., et al. (1993). Naturally occurring antibodies devoid of light chains. Nature, 363(6428): 446-448.

Harke, B. (2008). 3D STED Microscopy with Pulsed and Continuous Wave Lasers. Dissertation, University of Göttingen.

Harke, B., et al. (2008). Resolution scaling in STED microscopy. Opt Express, 16(6): 4154-4162.

Harke, B., Ullal, C. K., Keller, J. and Hell, S. W. (2008). Three-dimensional nanoscopy of colloidal crystals. Nano Lett, 8(5): 1309-1313.

Harris, K. M. and Stevens, J. K. (1989). Dendritic spines of CA 1 pyramidal cells in the rat hippocampus: serial electron microscopy with reference to their biophysical characteristics. J Neurosci, 9(8): 2982-2997.

Heilemann, M., et al. (2008). Subdiffraction-resolution fluorescence imaging with conventional fluorescent probes. Angew Chem, 47(33): 6172-6176.

Heim, R. and Tsien, R. Y. (1996). Engineering green fluorescent protein for improved brightness, longer wavelengths and fluorescence resonance energy transfer. Curr Biol, 6(2): 178-182.

Heimstadt, O. (1911). Das Fluoreszenzmikroskop. Z Wiss Mikrosk, 28: 330-337.

Hein, B., Willig, K. I. and Hell, S. W. (2008). Stimulated emission depletion (STED) nanoscopy of a fluorescent proteinlabeled organelle inside a living cell. P Natl Acad Sci USA, 105(38): 14271-14276.

Heine, J., et al. (2017). Adaptive-illumination STED nanoscopy. P Natl Acad Sci USA, 114(37): 9797-9802

Heintzmann, R. and Cremer, C. G. (1999). Lateral modulated excitation microscopy: Improvement of resolution by using a diffraction grating. Proc SPIE, 3568: 185-196.

Heintzmann, R., Jovin, T. M. and Cremer, C. (2002). Saturated patterned excitation microscopy - a concept for optical resolution improvement. J Opt Soc Am A, 19(8): 1599-1609.

Hell, S. and Stelzer, E. H. K. (1992). Properties of a 4Pi confocal fluorescence microscope. J Opt Soc Am A, 9(12): 2159-2166.

Hell, S. W. (1994). Improvement of lateral resolution in far-field fluorescence light microscopy by using two-photon excitation with offset beams. Opt Commun, 106(1-3): 19-24.

Hell, S. W. and Wichmann, J. (1994). Breaking the diffraction resolution limit by stimulated emission: stimulatedemission-depletion fluorescence microscopy. Opt Lett, 19(11): 780-782.

Hell, S. W. and Kroug, M. (1995). Ground-state-depletion fluorscence microscopy: a concept for breaking the diffraction resolution limit. Appl Phys B-Lasers O, 60(5): 495-497. 
Hell, S. W. (2003). Toward fluorescence nanoscopy. Nat Biotechnol, 21(11): 1347-1355.

Hell, S. W. (2007). Far-field optical nanoscopy. Science, 316(5828): 1153-1158.

Hell, S. W. (2009). Microscopy and its focal switch. Nat Methods, 6(1): 24-32.

Helmchen, F. and Denk, W. (2005). Deep tissue two-photon microscopy. Nat Methods, 2(12): 932-940.

Helmchen, F. (2009). Two-photon functional imaging of neuronal activity in In vivo optical imaging of brain function from Frostig, R. D.: CRC Press, Boca Raton, USA.

Henrich, M. B. (2014). The Potential of Semiconductor Lasers for STED Nanoscopy. Dissertation, University of Heidelberg.

Hense, A., et al. (2015). Monomeric Garnet, a far-red fluorescent protein for live-cell STED imaging. Sci Rep, 5: 18006.

Hering, H. and Sheng, M. (2001). Dendritic spines: structure, dynamics and regulation. Nat Rev Neurosci, 2(12): 880-888.

Herschel, J. (1845). On a case of superficial colour presented by a homogeneous liquid internally colourless. Phil Trans R Soc Lond, 135: 143-145.

Hess, S. T., Girirajan, T. P. K. and Mason, M. D. (2006). Ultra-high resolution imaging by fluorescence photoactivation localization microscopy. Biophys J, 91(11): 4258-4272.

Hofmann, M., Eggeling, C., Jakobs, S. and Hell, S. W. (2005). Breaking the diffraction barrier in fluorescence microscopy at low light intensities by using reversibly photoswitchable proteins. P Natl Acad Sci USA, 102(49): 17565-17569.

Holtmaat, A., et al. (2009). Long-term, high-resolution imaging in the mouse neocortex through a chronic cranial window. Nat Protoc, 4(8): 1128-1144.

Hooke, R. (1665). Micrographia: or, Some physiological descriptions of minute bodies, made by magnifying glasses with observations and inquiries thereupon. London, United Kingdom: The Royal Society.

Huganir, R. L. and Nicoll, R. A. (2013). AMPARs and Synaptic Plasticity: The Last 25 Years. Neuron, 80(3): 704-717.

Hussain, N. K. and Huganir, R. L. (2016). Structure and molecular organization of the postsynaptic density in Dendrites from Stuart, G., Spruston, N. and Häusser, M.: Oxford University Press, New York, USA.

Jabłoński, A. (1933). Efficiency of Anti-Stokes Fluorescence in Dyes. Nature, 131(3319): 839-840.

Kaech, S. and Banker, G. (2006). Culturing hippocampal neurons. Nat Protoc, 1(5): 2406-2415.

Karlsson Rosenthal, C. (2009). Milestone 1: The beginning. Nat Cell Biol, 11: S6.

Keller, J., Schönle, A. and Hell, S. W. (2007). Efficient fluorescence inhibition patterns for RESOLFT microscopy. Opt Express, 15(6): 3361-3371.

Kellner, R. R., Baier, C. J., Willig, K. I., Hell, S. W. and Barrantes, F. J. (2007). Nanoscale organization of nicotinic acetylcholine receptors revealed by stimulated emission depletion microscopy. Neuroscience, 144(1): 135-143.

Keppler, A., et al. (2003). A general method for the covalent labeling of fusion proteins with small molecules in vivo. Nat Biotechnol, 21(1): 86-89.

Kim, E. and Sheng, M. (2004). PDZ domain proteins of synapses. Nat Rev Neurosci, 5(10): 771-781.

Kistner, U., et al. (1993). SAP90, a rat presynaptic protein related to the product of the Drosophila tumor suppressor gene dlg-A. J Biol Chem, 268(7): 4580-4583.

Klar, T. A. and Hell, S. W. (1999). Subdiffraction resolution in far-field fluorescence microscopy. Opt Lett, 24(14): 954-956. 
Klar, T. A., Jakobs, S., Dyba, M., Egner, A. and Hell, S. W. (2000). Fluorescence microscopy with diffraction resolution limit broken by stimulated emission. P Natl Acad Sci USA, 97(15): 8206-8210.

König, K. (2000). Multiphoton microscopy in life sciences. J Microsc, 200(2): 83-104.

Kotlyar, V. V., et al. (2005). Generation of phase singularity through diffracting a plane or Gaussian beam by a spiral phase plate. J Opt Soc Am A, 22(5): 849-861

Kremers, G. J., Gilbert, S. G., Cranfill, P. J., Davidson, M. W. and Piston, D. W. (2011). Fluorescent proteins at a glance. J Cell Sci, 124(Pt2): 157-160.

Kubby, J. A. (2013). Adaptive Optics for Biological Imaging. CRC Press, Boca Raton, USA.

Kügler, S., Lingor, P., Schöll, U., Zolotukhin, S. and Bähr, M. (2003). Differential transgene expression in brain cells in vivo and in vitro from AAV-2 vectors with small transcriptional control units. Virology, 311(1): 89-95.

Kuzmenkov, A. I., et al. (2016). Fluorescent protein-scorpion toxin chimera is a convenient molecular tool for studies of potassium channels. Sci Rep, 6: 33314.

Lehmann, H. (1913). Das Lumineszenzmikroskop, seine Grundlagen und seine Anwendungen. Z Wiss Mikrosk, 30: 417-470.

Leica Microsystems (2014). Leica objectives - Superior optics for confocal and multiphoton research microscopy. Brochure: 1-24.

Lichtman, J. W. and Denk, W. (2011). The big and the small: challenges of imaging the brain's circuits. Science, 334: 618-623.

Liljeström, P. and Garoff, H. (1991). A new generation of animal cell expression vectors based on the Semliki Forest virus replicon. Bio/Technology, 9(12): 1356-1361.

Lin, M. Z., et al. (2009). Autofluorescent proteins with excitation in the optical window for intravital imaging in mammals. Chem Biol, 16(11): 1169-1179.

Liu, Y., et al. (2017). AgHalo: A Facile Fluorogenic Sensor to Detect Drug-Induced Proteome Stress. Angew Chem Int Ed, 56(30): 8672-8676.

Lodish, H., et al. (2000). Molecular Cell Biology, 4. New York, W. H. Freeman.

Los, G. V. and Wood, K. (2007). The HaloTag: a novel technology for cell imaging and protein analysis. Methods Mol Biol, 356: 195-208.

Los, G. V., et al. (2008). HaloTag: A Novel Protein Labeling Technology for Cell Imaging and Protein Analysis. ACS Chem Biol, 3(6): 373-382.

Lukinavičius, G., et al. (2013). A near-infrared fluorophore for live-cell super-resolution microscopy of cellular proteins. Nat Chem, 5(2): 132-139.

Lukinavičius, G., et al. (2014). Fluorogenic probes for live-cell imaging of the cytoskeleton. Nat Methods, 11(7): 731-733.

Lukinavičius, G., et al. (2015). SiR-Hoechst is a far-red DNA stain for live-cell nanoscopy. Nat Commun, 6: 8497.

Lukinavičius, G., et al. (2016). Fluorogenic Probes for Multicolor Imaging in Living Cells. J Am Chem Soc, 138(30): 9365-9368.

Lundstrom, K., Rotmann, D., Hermann, D., Schneider, E. M. and Ehrengruber, M. U. (2000). Novel mutant Semliki Forest virus vectors: gene expression and localization studies in neuronal cells. Histochemistry and Cell Biology, 115(1): 83-91.

Lundstrom, K., Abenavoli, A., Malgaroli, A. and Ehrengruber, M. U. (2003). Novel Semliki Forest virus vectors with reduced cytotoxicity and temperature sensitivity for long-term enhancement of transgene expression. Mol Ther, 7(2): 202-209. 
MacGillavry, H. D., Song, Y., Raghavachari, S. and Blanpied, T. A. (2013). Nanoscale scaffolding domains within the postsynaptic density concentrate synaptic AMPA receptors. Neuron, 78(4): 615-622.

Maglione, M. and Sigrist, S. J. (2013). Seeing the forest tree by tree: super-resolution light microscopy meets the neurosciences. Nat Neurosci, 16(7): 790-797.

Massensini, A. R., et al. (2002). Tracking sodium channels in live cells: confocal imaging using fluorescently labeled toxins. J Neurosci Meth, 116(2): 189-196.

Matela, G., et al. (2017). A far-red emitting fluorescent marker protein, mGarnet2, for microscopy and STED nanoscopy. Chem Commun, 53(5): 979-982.

Maurer, C., Jesacher, A., Bernet, S. and Ritsch-Marte, M. (2011). What spatial light modulators can do for optical microscopy. Laser Photonics Rev, 5(1): 81-101.

Minsky, M. (1961). Microscopy apparatus. US Patent 3013467 A.

Minsky, M. (1988). Memoir on inventing the confocal scanning microscope. Scanning, 10(4): 128-138.

Moffitt, J. R., Osseforth, C. and Michaelis, J. (2011). Time-gating improves the spatial resolution of STED microscopy. Opt Express, 19(5): 4242-4254.

Morozova, K. S., et al. (2010). Far-red fluorescent protein excitable with red lasers for flow cytometry and superresolution STED nanoscopy. Biophys J, 99(2): L13-L15.

Müller, V., et al. (2011). STED nanoscopy reveals molecular details of cholesterol-and cytoskeleton-modulated lipid interactions in living cells. Biophys J, 101(7): 1651-1660.

Muyldermans, S. J. (2001). Single domain camel antibodies: current status. J Biotechnol, 74(4): 277-302.

Nägerl, U. V., Willig, K. I., Hein, B., Hell, S. W. and Bonhoeffer, T. (2008). Live-cell imaging of dendritic spines by STED microscopy. P Natl Acad Sci USA, 105(48): 18982-18987.

Nair, D., et al. (2013). Super-resolution imaging reveals that AMPA receptors inside synapses are dynamically organized in nanodomains regulated by PSD95. J Neurosci, 33(32): 13204-13224.

Nassi, J. J., Cepko, C. L., Born, R. T. and Beier, K. T. (2015). Neuroanatomy goes viral! Front Neuroanat, 9: 80.

Nicoll, R. A., Tomita, S. and Bredt, D. S. (2006). Auxiliary subunits assist AMPA-type glutamate receptors. Science, 311(5765): 1253-1256.

Nienhaus, K. and Nienhaus, G. U. (2014). Fluorescent proteins for live-cell imaging with super-resolution. Chem Soc Rev, 43(4): 1088-1106.

Niethammer, M., Kim, E. and Sheng, M. (1996). Interaction between the C terminus of NMDA receptor subunits and multiple members of the PSD-95 family of membrane-associated guanylate kinases. J Neurosci, 16(7):2157-2163.

Nieto-Sampedro, M., Hoff, S. F. and Cotman, C. W. (1982). Perforated Postsynaptic Densities: Probable Intermediates in Synapse Turnover. P Natl Acad Sci USA, 79(18): 5718-5722.

Nimchinsky, E. A., Sabatini, B. L. and Svoboda, K. (2002). Structure and function of dendritic spines. Annu Rev Physiol, 64: 313-353.

Nomarski, G. (1955). Microinterféromètre différentiel à ondes polarisées. J Phys Radium, 16: 9S-11S.

O'Brien, R. J., Lau, L. F. and Huganir, R. L. (1998). Molecular mechanisms of glutamate receptor clustering at excitatory synapses. Curr Opin Neurobiol, 8(3): 364-369.

Ondrus, A. E., et al. (2012). Fluorescent saxitoxins for live cell imaging of single voltage-gated sodium ion channels beyond the optical diffraction limit. Chem Biol, 19(7): 902-912.

Osseforth, C., Moffitt, J. R., Schermelleh, L. and Michaelis, J. (2014). Simultaneous dual-color 3D STED microscopy. Opt Express, 22(6): 7028-7039. 
Palay, S. L. (1956). Synapses in the central nervous system. J Biophys Biochem Cy, 2(4): 193-202.

Palay, S. L. (1958). The morphology of synapses in the central nervous system. Exp Cell Res, 14(Suppl 5): 275-293.

Papa, M., Bundman, M. C., Greenberger, V. and Segal, M. (1995). Morphological analysis of dendritic spine development in primary cultures of hippocampal neurons. J Neurosci, 15(1): 1-11.

Papouin, T., Dunphy, J., Tolman, M., Foley, J. C. and Haydon, P. G. (2017). Astrocytic control of synaptic function. Philos TRoy Soc B, 372(1715): 20160154.

Patterson, G. H., Knobel, S. M., Sharif, W. D., Kain, S. R. and Piston, D. W. (1997). Use of the green fluorescent protein and its mutants in quantitative fluorescence microscopy. Biophys J, 73(5): 2782-2790.

Patton, B. R., Burke, D., Vrees, R. and Booth, M. J. (2015). Is phase-mask alignment aberrating your STED microscope? Methods Appl Fluores, 3(2): 024002.

Patton, B. R., et al. (2016). Three-dimensional STED microscopy of aberrating tissue using dual adaptive optics. Opt Express, 24(8): 8862-8876.

Pawley, J. B. (2006). Handbook of Biological Confocal Microscopy, 3rd edition. Springer, New York, USA.

Paxinos, G. and Franklin, K. (2007). The Mouse Brain in Stereotaxic Coordinates, 3rd edition. Elsevier, New York, USA.

Peel, A. L. and Klein, R. L. (2000). Adeno-associated virus vectors: activity and applications in the CNS. J Neurosci Meth, 98(2): 95-104.

Peters, A., Palay, S. L. and Webster, H. D. (1991). The fine structure of the nervous system: neurons and their supporting cells. Oxford University Press, New York, USA.

Prasher, D. C., Eckenrode, V. K., Ward, W. W., Prendergast, F. G. and Cormier, M. J. (1992). Primary structure of the aequorea-victoria green-fluorescent protein. Gene, 111(2): 229-233.

Rankin, B. R., et al. (2011). Nanoscopy in a living multicellular organism expressing GFP. Biophys J, 100(12): L63-L65.

Reichert, K. (1911). Das Fluoreszenzmikroskop. Phys Z, 12: 1010-1011.

Reindl, S. and Penzkofer, A. (1996). Higher excited-state triplet-singlet intersystem crossing of some organic dyes. Chem Phys, 211(1): 431-439.

Reuss, M., Engelhardt, J. and Hell, S. W. (2010). Birefringent device converts a standard scanning microscope into a STED microscope that also maps molecular orientation. Opt Express, 18(2): 1049-1058.

Richards, B. and Wolf, E. (1959). Electromagnetic diffraction in optical systems. II. Structure of the image field in an aplanatic system. Proc R Soc Lond A Mat, 253(1274): 358-379.

Richardson, W. H. (1972). Bayesian-Based Iterative Method of Image Restoration. J Opt Soc Am, 62(1): 55-59.

Riedl, J., et al. (2008). Lifeact: a versatile marker to visualize F-actin. Nat Methods, 5(7): 605-607.

Rothbauer, U., et al. (2006). Targeting and tracing antigens in live cells with fluorescent nanobodies. Nat Methods, 3(11): 887-889.

Rust, M. J., Bates, M. and Zhuang, X. (2006). Sub-diffraction-limit imaging by stochastic optical reconstruction microscopy (STORM). Nat Methods, 3(10): 793-796.

Saka, S. K., et al. (2014). Multi-protein assemblies underlie the mesoscale organization of the plasma membrane. Nat Commun, 25: 4509.

Schmidt, R., et al. (2008). Spherical nanosized focal spot unravels the interior of cells. Nat Methods, 5(6): 539-544.

Schneider, J., et al. (2015). Ultrafast, temporally stochastic STED nanoscopy of millisecond dynamics. Nat Methods, 12(9): 827-830. 
Schönle, A. (2006). "Imspector Image Acquisition \& Analysis Software, v0.10." from http://www.imspector.de.

Schrader, M., et al. (1995). Monitoring the excited state of a fluorophore in a microscope by stimulated emission. Bioimaging, 3(4): 147-153.

Shaner, N. C., Steinbach, P. A. and Tsien, R. Y. (2005). A guide to choosing fluorescent proteins. Nat Methods, 2(12): 905-909.

Sharonov, A. and Hochstrasser, R. M. (2006). Wide-field subdiffraction imaging by accumulated binding of diffusing probes. P Natl Acad Sci USA, 103(50): 18911-18916.

Shaw, P. J. and Rawlins, D. J. (1991). The point-spread function of a confocal microscope: its measurement and use in deconvolution of 3-D data. J Microsc, 163(2): 151-165.

Shcherbo, D., et al. (2007). Bright far-red fluorescent protein for whole-body imaging. Nat Methods, 4(9): 741-746.

Shcherbo, D., et al. (2009). Far-red fluorescent tags for protein imaging in living tissues. Biochem J, 418(3): 567-774.

Sheng, M. and Hoogenraad, C. C. (2007). The postsynaptic architecture of excitatory synapses: a more quantitative view. Annu Rev Biochem, 76: 823-847.

Sheppard, C. J. and Wilson, T. (1981). The theory of the direct-view confocal microscope. J Microsc, 124(Pt 2): 107-117.

Shevtsova, Z., Malik, J. M., Michel, U., Bahr, M. and Kügler, S. (2005). Promoters and serotypes: targeting of adenoassociated virus vectors for gene transfer in the rat central nervous system in vitro and in vivo. Exp Physiol, 90(1): 53-59.

Sidenstein, S. C., et al. (2016). Multicolour multilevel STED nanoscopy of actin/spectrin organization at synapses. Sci Rep, 6: 26725.

Sigrist, S. J. and Sabatini, B. L. (2012). Optical super-resolution microscopy in neurobiology. Curr Opin Neurobiol, 22(1): 86-93.

Staudt, T., et al. (2011). Far-field optical nanoscopy with reduced number of state transition cycles. Opt Express, 19(6): 5644-5657.

Stein, V., House, D. R., Bredt, D. S. and Nicoll, R. A. (2003). Postsynaptic density-95 mimics and occludes hippocampal long-term potentiation and enhances long-term depression. J Neurosci, 23(13): 5503-5506.

Stewart, M. G., et al. (2005). Chemically induced long- term potentiation increases the number of perforated and complex postsynaptic densities but does not alter dendritic spine volume in CA1 of adult mouse hippocampal slices. Eur J Neurosci, 21(12): 3368-3378.

Stokes, G. (1852). On the change of refractibility of light. Phil Trans R Soc Lond, 142: 463-562.

Sugiyama, Y., Kawabata, I., Sobue, K. and Okabe, S. (2005). Determination of absolute protein numbers in single synapses by a GFP-based calibration technique. Nat Methods, 2(9): 677-684.

Svoboda, K. and Block, S. M. (1994). Biological applications of optical forces. Annu Rev Bioph Biomol Struct, 23: 247-285.

Svoboda, K., Denk, W., Kleinfeld, D. and Tank, D. W. (1997). In vivo dendritic calcium dynamics in neocortical pyramidal neurons. Nature, 385(6612): 161-165.

Tang, A.-H., et al. (2016). A trans-synaptic nanocolumn aligns neurotransmitter release to receptors. Nature, 536(7615): 210-214.

Tønnesen, J., Nadrigny, F., Willig, K. I., Wedlich-Soldner, R. and Nägerl, U. V. (2011). Two-color STED microscopy of living synapses using a single laser-beam pair. Biophys J, 101(10): 2545-2552.

Tønnesen, J. and Nägerl, U. V. (2013). Superresolution imaging for neuroscience. Exp Neurol, 242: 33-40. 
Trachtenberg, J. T., et al. (2002). Long-term in vivo imaging of experience-dependent synaptic plasticity in adult cortex. Nature, 420(6917): 788-794.

Urban, N. T., Willig, K. I., Hell, S. W. and Nägerl, U. V. (2011). STED nanoscopy of actin dynamics in synapses deep inside living brain slices. Biophys J, 101(5): 1277-1284.

van Leeuwenhoek, A. (1673). A specimen of some observations made by a microscope. Phil Trans, 8(92-100): 6037-6038.

Vicidomini, G., et al. (2013). STED nanoscopy with time-gated detection: theoretical and experimental aspects. PLoS One, 8(1): e54421.

von Helmholtz, H. (1874). Die theoretische Grenze für die Leistungsfahigkeit der Mikroskope. Ann Phys Chem, (Jubelband, J. C. Poggendorff gewidmet): 557-584.

Wäldchen, S., Lehmann, J., Klein, T., van de Linde, S. and Sauer, M. (2015). Light-induced cell damage in live-cell super-resolution microscopy. Sci Rep, 5: 15348.

Wang, L., Jackson, W., Steinbach, P. and Tsien, R. (2004). Evolution of new nonantibody proteins via iterative somatic hypermutation. P Natl Acad Sci USA, 101(48): 16745-16749.

Weissleder, R. (2001). A clearer vision for in vivo imaging. Nat Biotechnol, 19(4): 316-317.

Westphal, V. and Hell, S. W. (2005). Nanoscale resolution in the focal plane of an optical microscope. Phys Rev Lett, 94(14): 143903.

Westphal, V., et al. (2008). Video-rate far-field optical nanoscopy dissects synaptic vesicle movement. Science, 320(5873): 246-249.

Whelan, D. R. and Bell, T. D. M. (2015). Image artifacts in Single Molecule Localization Microscopy: why optimization of sample preparation protocols matters. Sci Rep, 5: 7924.

Wildanger, D., Rittweger, E., Kastrup, L. and Hell, S. W. (2008). STED microscopy with a supercontinuum laser source. Opt Express, 16(13): 9614-9621.

Wildanger, D., Medda, R., Kastrup, L. and Hell, S. W. (2009). A compact STED microscope providing 3D nanoscale resolution. J MicrosC, 236(1): 35-43.

Wildanger, D., et al. (2012). Solid immersion facilitates fluorescence microscopy with nanometer resolution and sub-Ångström emitter localization. Adv Mater, 24(44): OP309-OP313.

Willig, K. I., et al. (2006). Nanoscale resolution in GFP-based microscopy. Nat Methods, 3(9): 721-723.

Willig, K. I., Rizzoli, S. O., Westphal, V., Jahn, R. and Hell, S. W. (2006). STED microscopy reveals that synaptotagmin remains clustered after synaptic vesicle exocytosis. Nature, 440(7086): 935-939.

Willig, K. I. and Barrantes, F. J. (2014). Recent applications of superresolution microscopy in neurobiology. Curr Opin Chem Biol, 20: 16-21.

Willig, K. I., et al. (2014). Nanoscopy of Filamentous Actin in Cortical Dendrites of a Living Mouse. Biophys J, 106(1): L01-L03.

Wilson, T. and Sheppard, C. J. R. (1984). Theory and practice of scanning optical microscopy. Academic Press, London, United Kingdom.

Winter, F. R., et al. (2017). Multicolour nanoscopy of fixed and living cells with a single STED beam and hyperspectral detection. Sci Rep, 7: 46492.

Xue, L., Karpenko, I. A., Hiblot, J. and Johnsson, K. (2015). Imaging and manipulating proteins in live cells through covalent labeling. Nat Chem Biol, 11(12): 917-923.

Yan, Q. and Bruchez, M. P. (2015). Advances in chemical labeling of proteins in living cells. Cell Tissue Res, 360(1): 179-194. 
Yang, G., et al. (2015). Genetic targeting of chemical indicators in vivo. Nat Methods, 12(2): 137-139.

Zernicke, F. (1934). Beugungstheorie des Schneidenverfahrens und seiner verbesserten Form, der Phasenkontrastmethode. Physica, 1(7): 689-704.

Zernicke, F. (1935). Das Phasenkontrastverfahren bei der mikroskopischen Beobachtung. Z technische Physik, 16: 454-457.

Zipfel, W. R., Williams, R. M. and Webb, W. W. (2003). Nonlinear magic: multiphoton microscopy in the biosciences. Nat Biotechnol, 21(11): 1369-1377. 


\section{A APPENDIX}

\section{A.1 Pictures of the STED nanoscope}

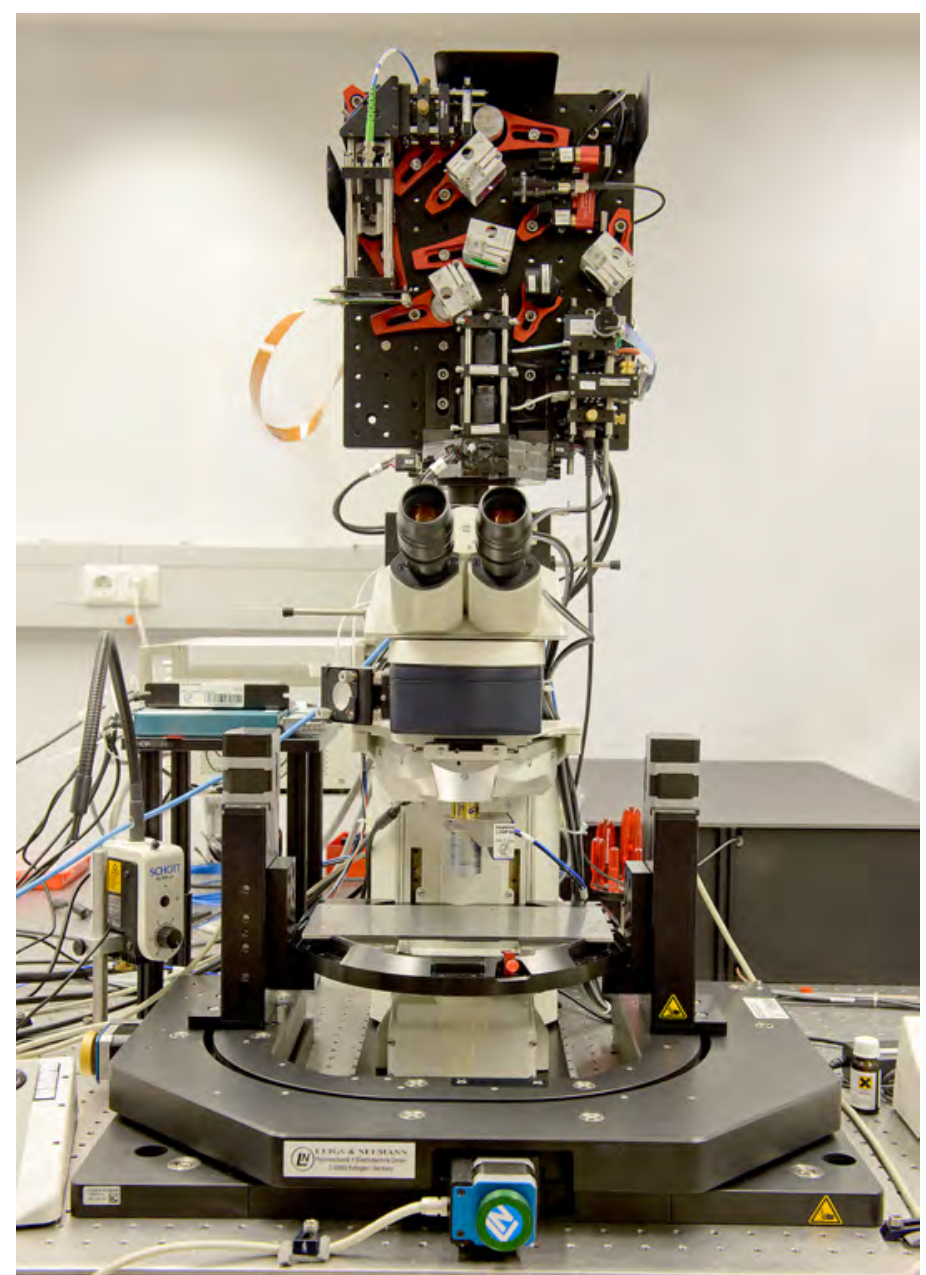

Figure A.1: Overview of the custom-built compact STED nanoscope

The minimized STED unit $(25 \mathrm{~cm} \times 30 \mathrm{~cm})$ was directly mounted to the camera port on top of a commercial microscope stand. The heavy and robust motorized translation stage was independently assembled around the microscope stand, providing enough space for the mouse and required support equipment (e.g. heating plate). Note that an earlier configuration (without the additional mechanical supports) is shown here to better see the details of the microscope. 


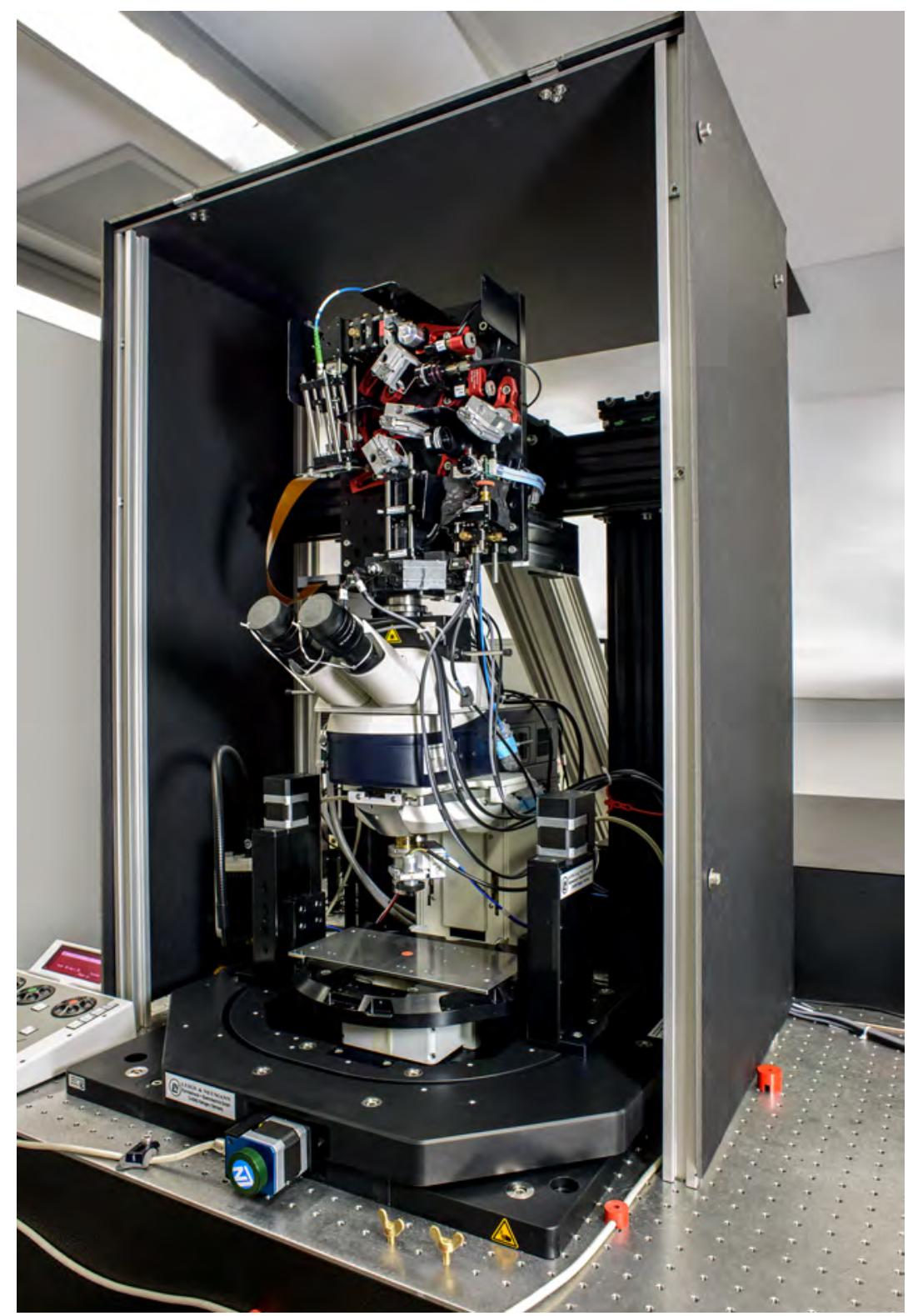

Figure A.2: Final configuration of the STED nanoscope with the additional mechanical supports

The STED unit on top of the microscope stand was stabilized against vibrations with respect to the microscope stand by implementing an additional mechanical construction, consisting of connected rails. A protective cover was assembled around the mechanical construction to block undesired light, acoustic noise, and air flow from the environment (here opened to allow a better perspective of the STED nanoscope).

\section{A.2 Analysis of gated and non-gated detection in STED nanoscopy}

For a comparison of the time-gated and non-gated detection, we acquired two images from $40 \mathrm{~nm}$ sized crimson bead samples on the same field-of-view $(10 \mu \mathrm{m} \times 10 \mu \mathrm{m})$. Imaging parameters were kept the same except for the time gate delay and time gate width of the detection. To analyze both images by comparing their effective PSFs, we processed the images with a MATLAB script to obtain the average intensity profile of several beads for both detection modes. In a first step, we identified the positions of single beads by finding local intensity maxima of a smoothed image and excluded too dim, too bright, or too dense clustered beads. The positions were estimated with sub-pixel accuracy after calculating the center-of-mass for every selected bead. In the next step, we superimposed the selected beads $(n=125)$ so that the calculated center-of-mass was used as the center for the overlaid patches. Image interpolation based on the nearest neighbor method was 
performed to calculate the overlaid image on a finer grid. As a result, we obtained averaged 2D PSFs for both detection modes. For the gated data, we approximated the average intensity profile with a single 2D circular Gaussian peak function. Due to the diffraction-limited background in the nongated data, we approximated this average intensity profile with two centered 2D circular Gaussian peak functions of different widths: one for the diffraction-unlimited intensity signal and one for the diffraction-limited background signal. The latter was caused by spontaneous emission from unquenched dye molecules while the depletion laser pulse did not yet act fully on the sample.

Figure A.3 compares the fitted lateral intensity profiles for the gated (green solid line) and non-gated (red solid line) effective PSFs. The fitted lateral intensity profile of the non-gated data is a superposition of the diffraction-unlimited intensity signal (red dashed line) and the diffractionlimited background signal (red dotted line). Time-gated detection reduces the entire signal intensity, where the pedestals are almost exclusively affected. No diffraction-limited background is apparent in the intensity profile of the gated data. To quantify the effect of time-gated detection in terms of fluorescence signal loss, we compared only the diffraction-unlimited intensity signals (gated STED: green solid line, non-gated STED: red dashed line). For gated STED, the fluorescence signal is decreased only by $2 \%$ in comparison to the non-gated (diffraction-unlimited) signal. As expected, the spatial resolution, determined by the FWHM, is the same. However, the actual benefit of using time-gated detection lies in the background reduction. The diffraction-limited background signal represents $44 \%$ of the total non-gated intensity signal and considerably impairs the image contrast. Due to the minor loss of fluorescence signal and significantly enhanced signal-to-background ratio, we decided to implement the time-gated detection in the custom-built pulsed STED nanoscope.

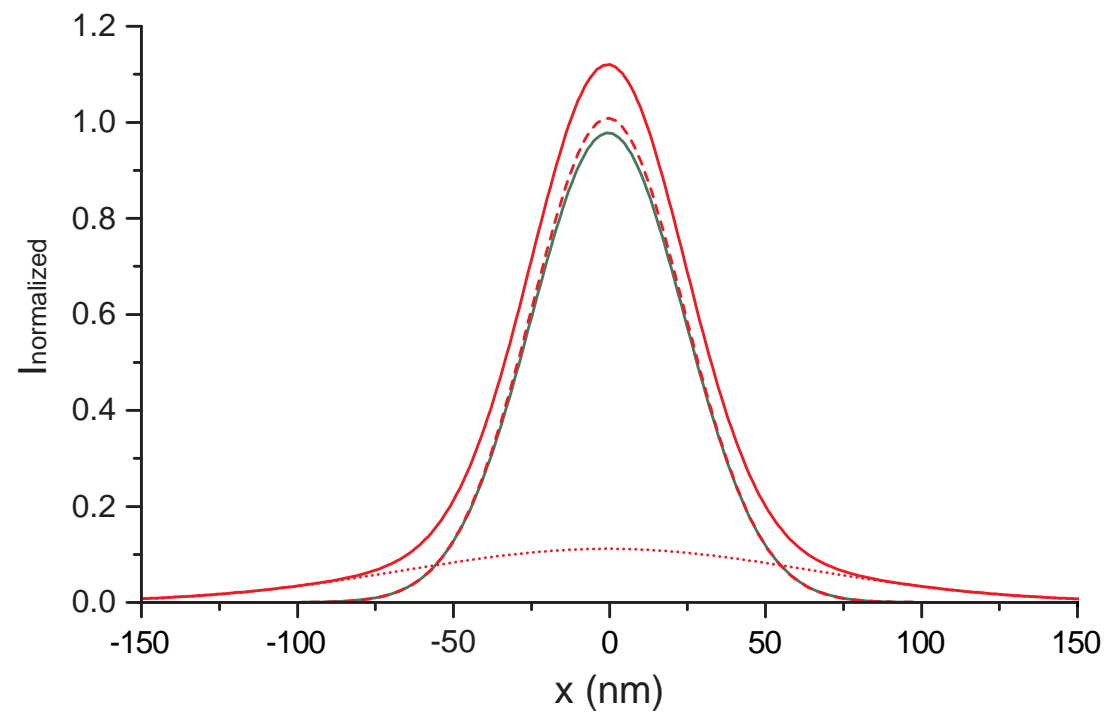

Figure A.3: PSF of gated and non-gated STED nanoscopy

Fitted lateral intensity distributions for the gated (green solid line) and non-gated (red solid line) effective PSF are shown. The latter is a superposition of the diffraction-unlimited intensity signal (red dashed line) and the diffraction-limited background signal (red dotted line). By using time-gated detection, the diffraction-unlimited signal is slightly reduced, whereas diffraction-limited signal is eliminated. The intensity distributions are normalized to the diffraction-unlimited intensity signal from the non-gated data. 


\section{A.3 Confocal PSF calculations}

To estimate the theoretical values of lateral and axial resolution in confocal mode of the STED nanoscope, we used a MATLAB routine ${ }^{44}$, based on Richards-Wolf diffraction integrals (Richards and Wolf, 1959). We calculated 3D PSFs by using the following parameters:

$\begin{array}{ll}\text { numerical aperture } & 1.3 \\ \text { excitation wavelengths } & 473 / 594 / 640 \mathrm{~nm} \\ \text { beam diameter } & \text { homogeneous infinite } \\ \text { excitation polarization } & \text { circular } \\ \text { emission wavelengths } & 525 / 623 / 685 \mathrm{~nm} \\ \text { lens immersion refractive index } & 1.45 \\ \text { pinhole size } & 1.2 \text { Airy units for } 685 \mathrm{~nm}\end{array}$

The specimen medium refractive index was neglected because structures attached to the coverslip were only considered for the experimental PSF measurements. Figure A.4 shows $x y$ - and $y z$-views of the calculated 3D PSFs for the three excitation-detection combinations with the relative FWHMs.
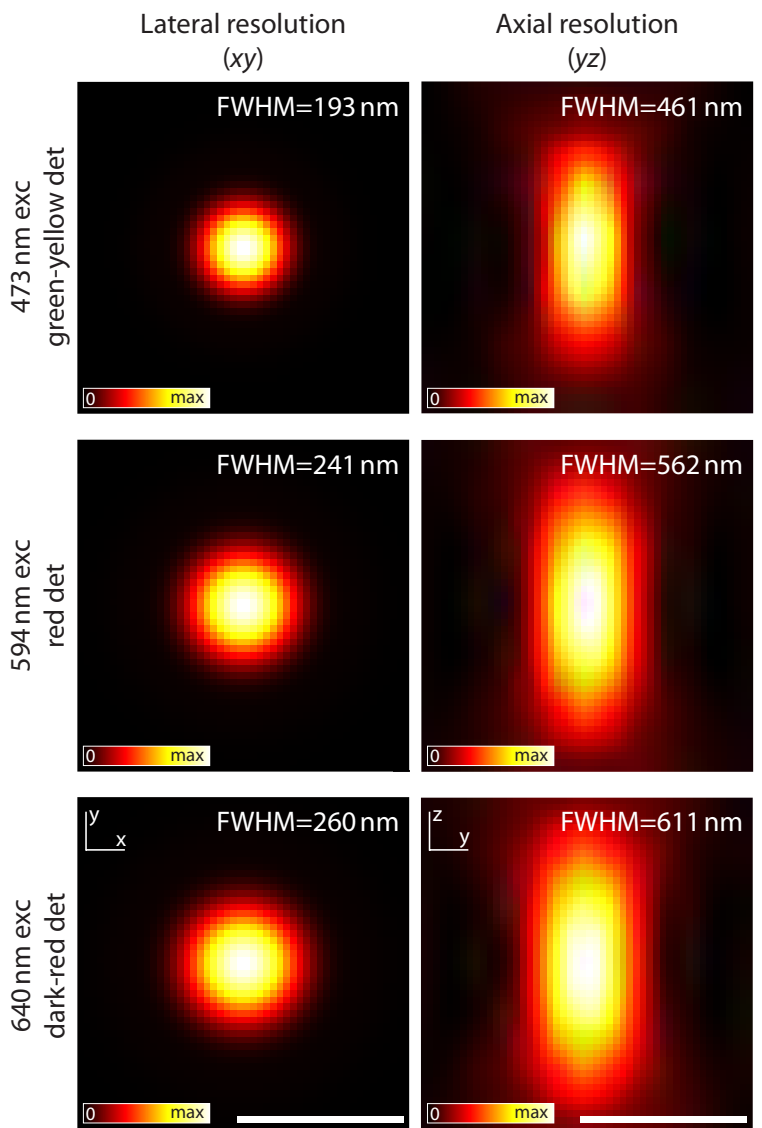

Figure A.4: Calculated intensity distributions in $x y$ - and $y z$-direction for each excitation-detection combination

Lateral (left column) and axial (right column) resolutions were determined by FWHM of a Gaussian curve fitting to the corresponding normalized intensity profiles (averaged over three pixels perpendicular to the direction of the profile) through the center of the calculated PSF. Scale bars: $500 \mathrm{~nm}$.

\footnotetext{
${ }^{44}$ Custom-written by Jan Keller-Findeisen, Max Planck Institute for Biophysical Chemistry, Göttingen, Germany
} 


\section{A.4 In vivo STED nanoscopy of PSD-95 distribution and substructure}

PSD-95-HaloTag with SiR-Halo (confocal/STED) Lifeact-YFP (confocal)
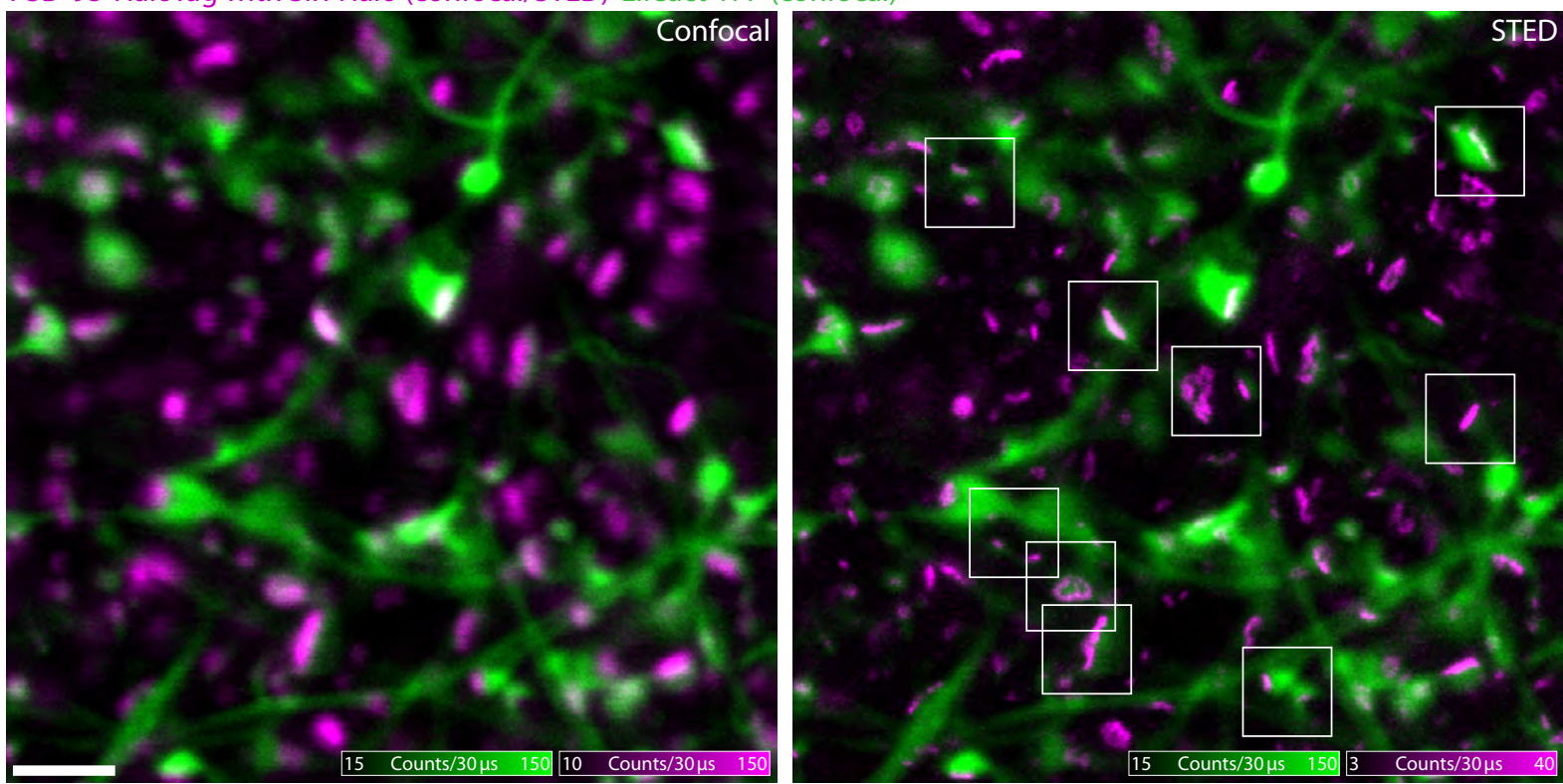

Figure A.5: In vivo STED nanoscopy of endogenous PSD-95 in dendrites in the molecular layer of the visual cortex of a mouse

Confocal (left) and STED (right) images of endogenous PSD-95-HaloTag (magenta) in cortical dendrites, stained with $750 \mathrm{nM}$ SiR-Halo-ligand. The STED and the confocal images were overlaid the same confocal image of Lifeact-YFP (green), which served as morphological reference labeling. All images were smoothed with a one pixel wide 2D Gaussian filter. Scale bar: $2 \mu \mathrm{m}$.

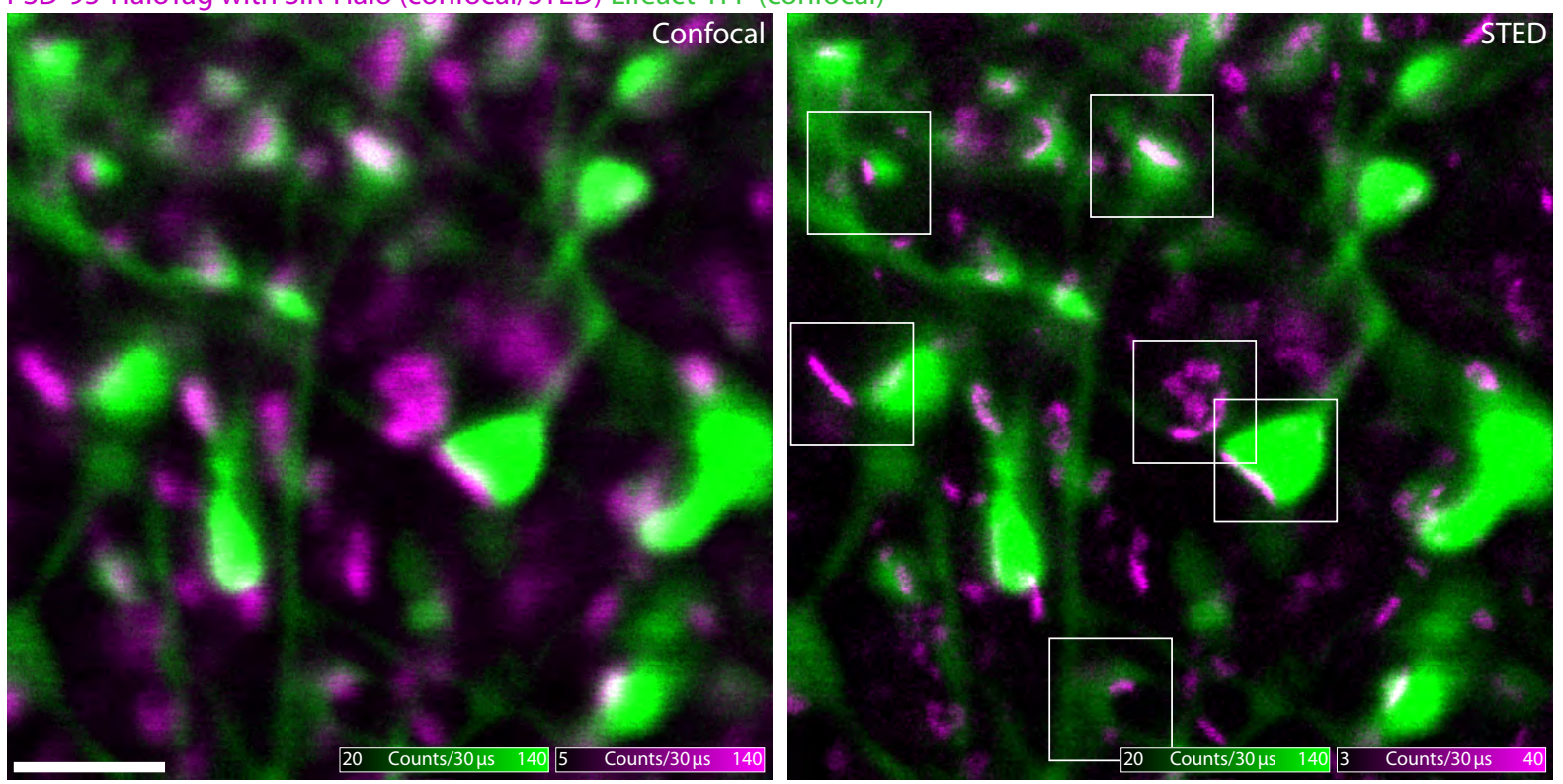

Figure A.6: In vivo STED nanoscopy of endogenous PSD-95 in dendrites in the molecular layer of the visual cortex of a mouse

Confocal (left) and STED (right) images of endogenous PSD-95-HaloTag (magenta) in cortical dendrites, stained with $750 \mathrm{nM}$ SiR-Halo-ligand. The STED and the confocal images were overlaid the same confocal image of Lifeact-YFP (green), which served as morphological reference labeling. All images were smoothed with a one pixel wide 2D Gaussian filter. Scale bar: $2 \mu \mathrm{m}$. 


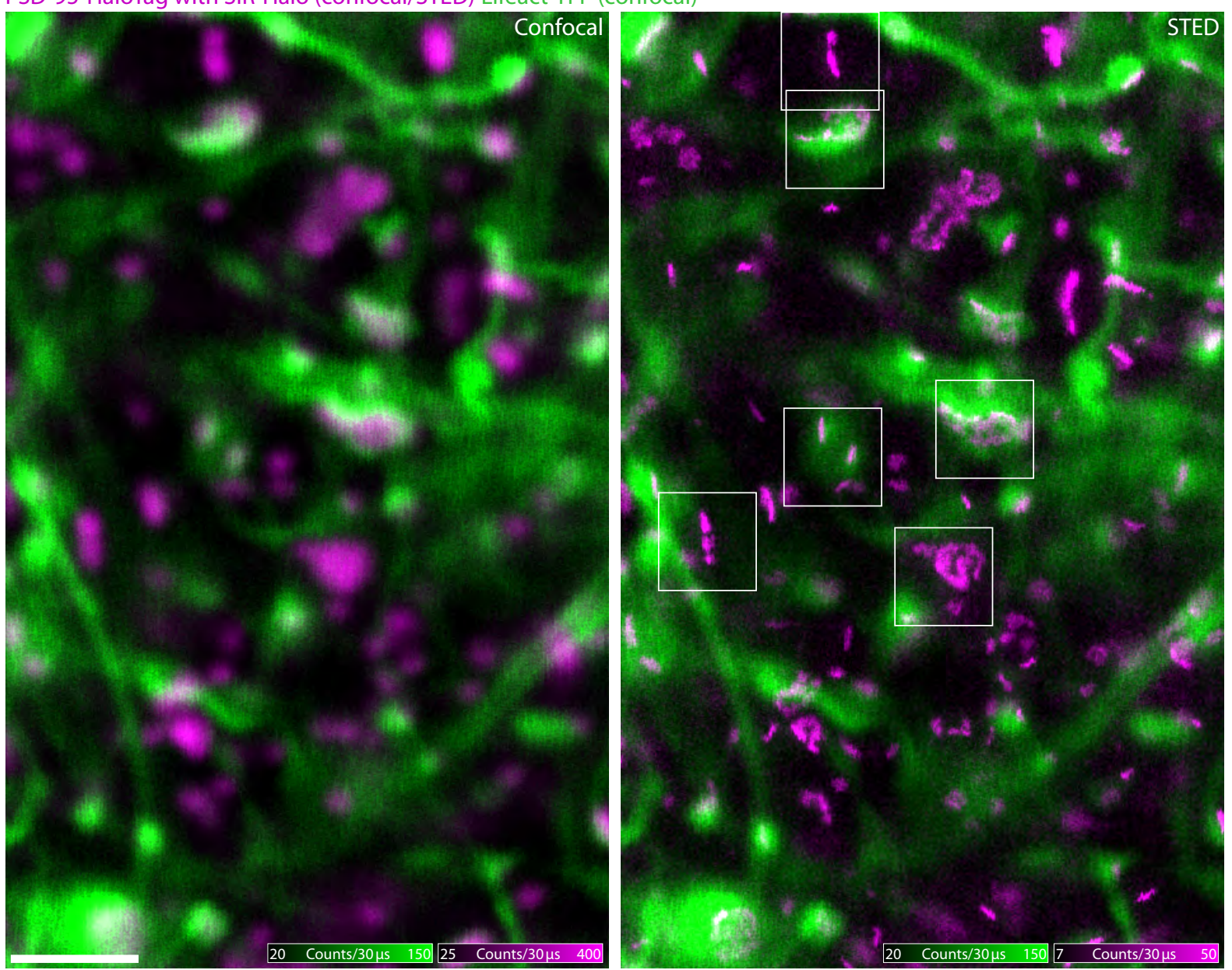

Figure A.7: In vivo STED nanoscopy of endogenous PSD-95 in dendrites in the molecular layer of the visual cortex of a mouse

Confocal (left) and STED (right) images of endogenous PSD-95-HaloTag (magenta) in cortical dendrites, stained with $750 \mathrm{nM}$ SiR-Halo-ligand. The STED and the confocal images were overlaid the same confocal image of Lifeact-YFP (green), which served as morphological reference labeling. All images were smoothed with a one pixel wide 2D Gaussian filter. Scale bar: $2 \mu \mathrm{m}$. 


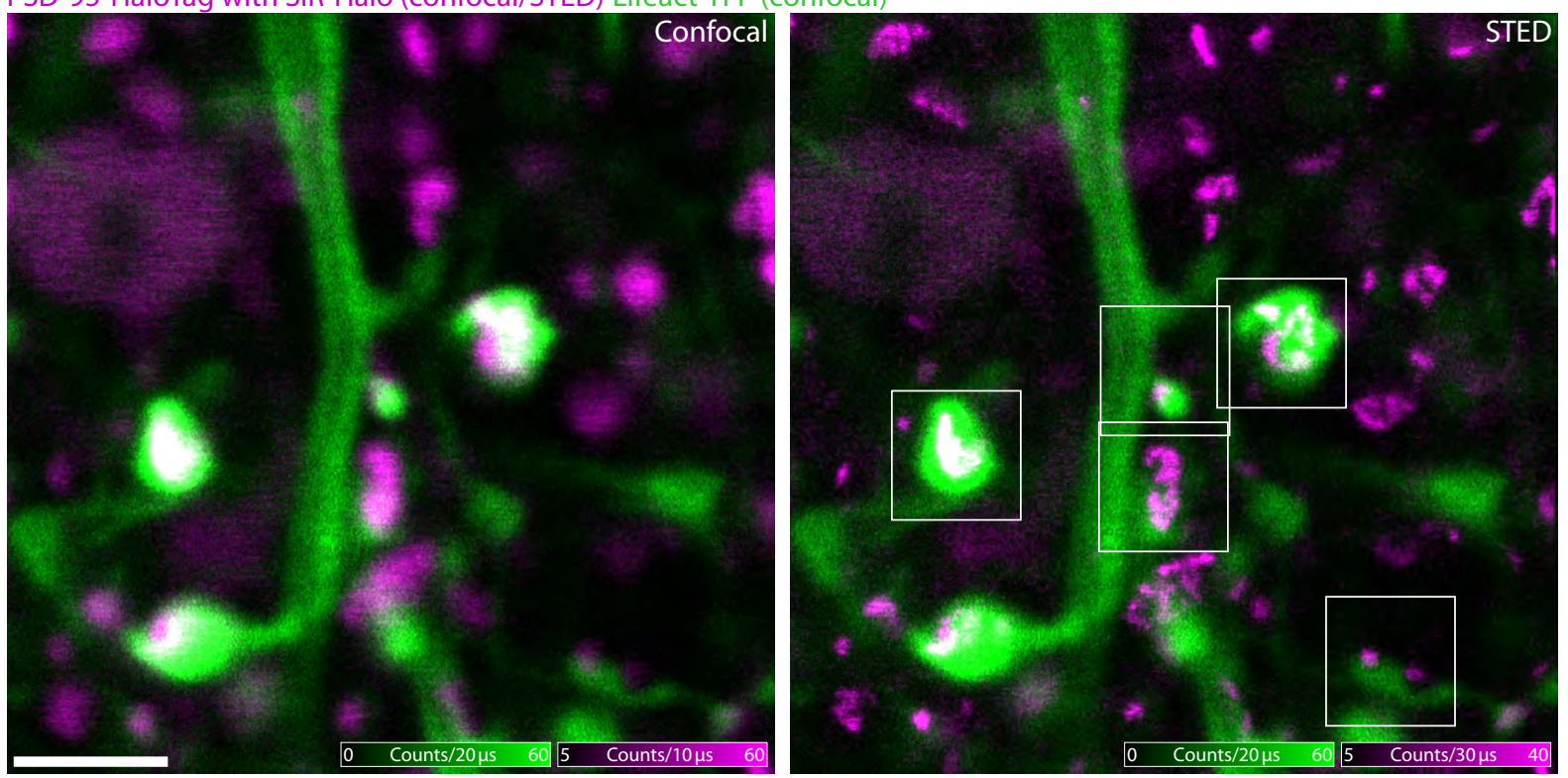

Figure A.8: In vivo STED nanoscopy of endogenous PSD-95 in dendrites in the molecular layer of the visual cortex of a mouse

Confocal (left) and STED (right) images of endogenous PSD-95-HaloTag (magenta) in cortical dendrites, stained with $750 \mathrm{nM}$ SiR-Halo-ligand. The STED and the confocal images were overlaid the same confocal image of Lifeact-YFP (green), which served as morphological reference labeling. All images were smoothed with a one pixel wide 2D Gaussian filter. Scale bar: $2 \mu \mathrm{m}$.

PSD-95-HaloTag with SiR-Halo (confocal/STED) Lifeact-YFP (confocal)

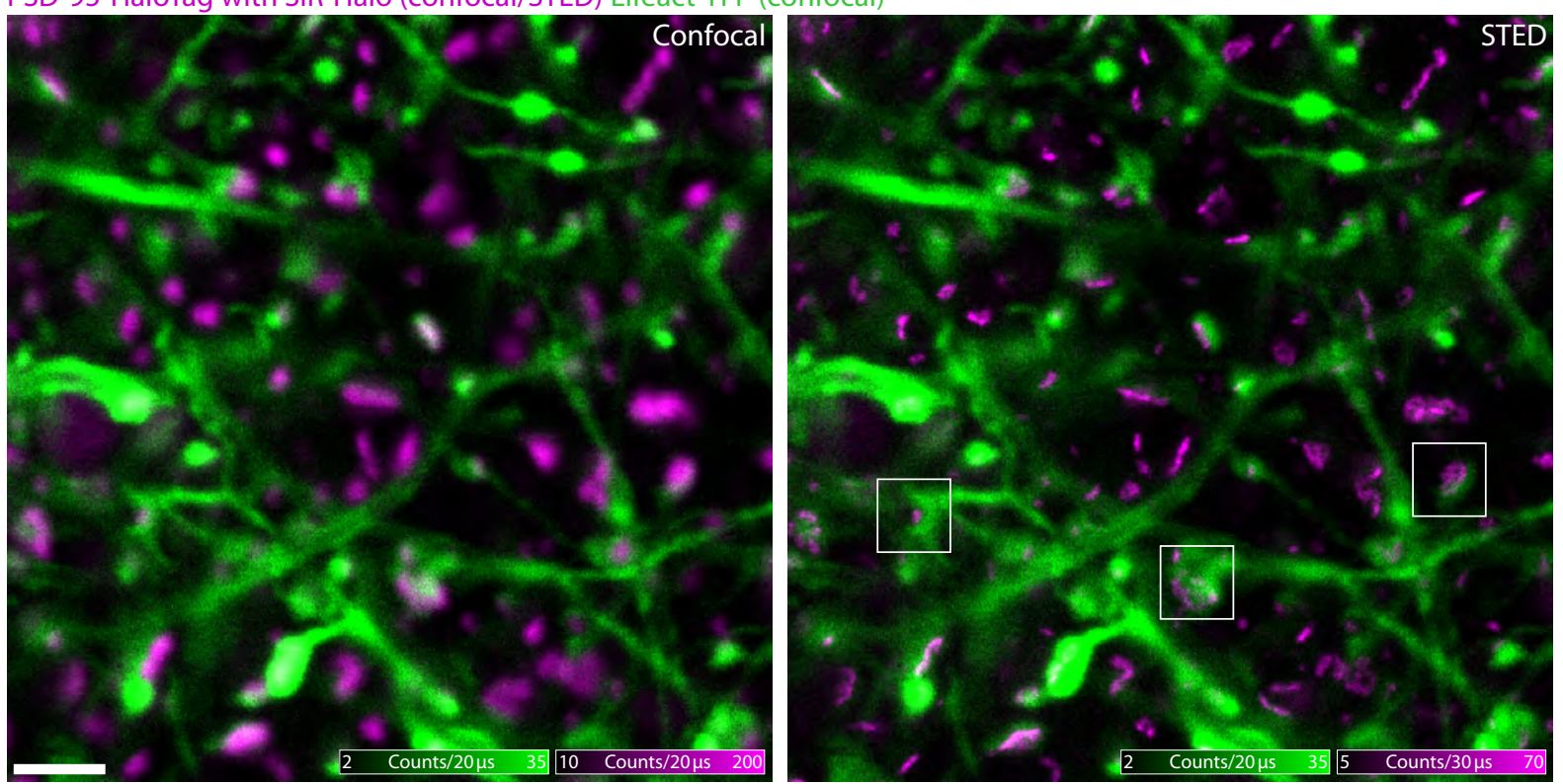

Figure A.9: In vivo STED nanoscopy of endogenous PSD-95 in dendrites in the molecular layer of the visual cortex of a mouse

Confocal (left) and STED (right) images of endogenous PSD-95-HaloTag (magenta) in cortical dendrites, stained with $750 \mathrm{nM}$ SiR-Halo-ligand. The STED and the confocal images were overlaid the same confocal image of Lifeact-YFP (green), which served as morphological reference labeling. All images were smoothed with a one pixel wide 2D Gaussian filter. Scale bar: $2 \mu \mathrm{m}$. 


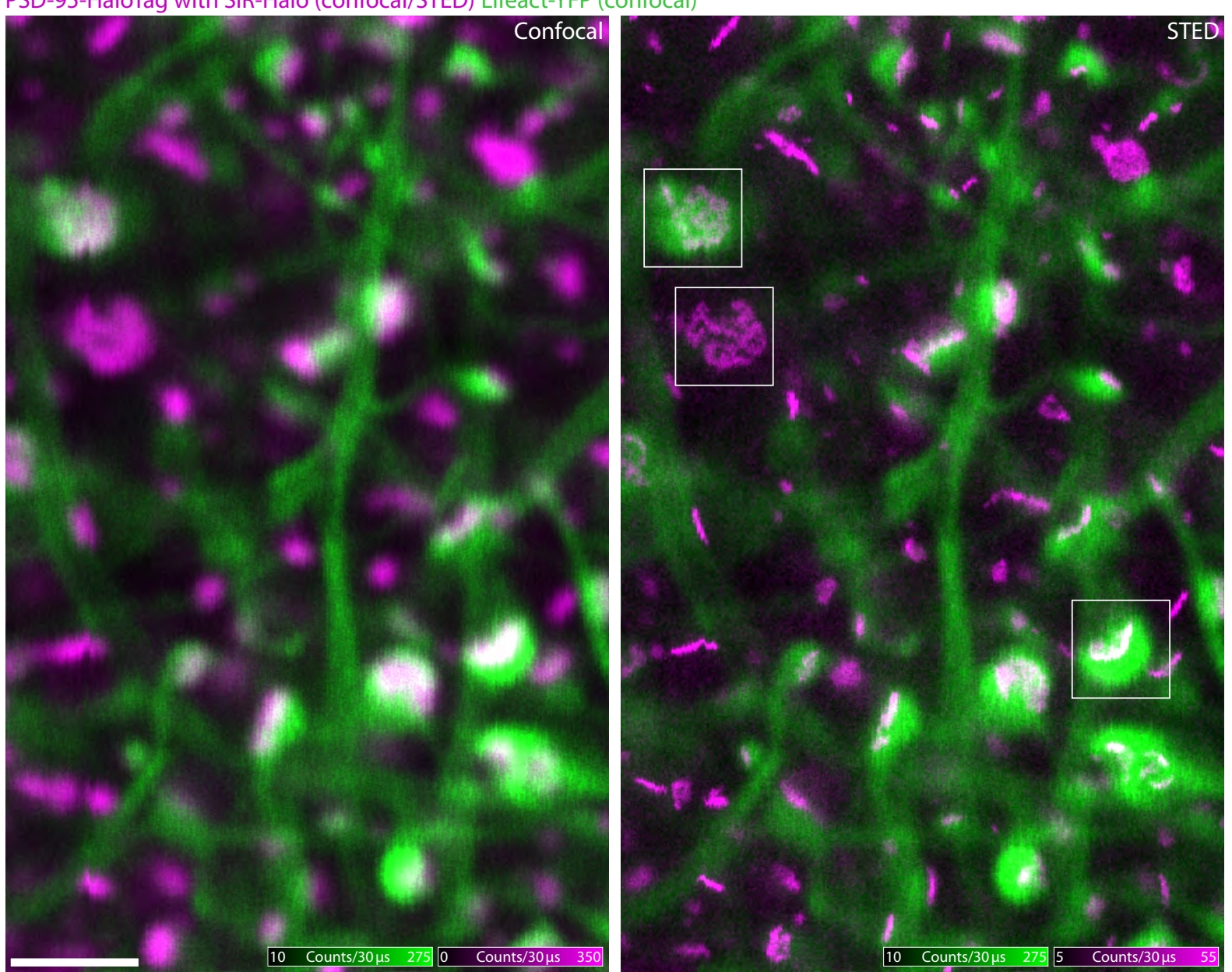

Figure A.10: In vivo STED nanoscopy of endogenous PSD-95 in dendrites in the molecular layer of the visual cortex of a mouse

Confocal (left) and STED (right) images of endogenous PSD-95-HaloTag (magenta) in cortical dendrites, stained with $750 \mathrm{nM}$ SiR-Halo-ligand. The STED and the confocal images were overlaid the same confocal image of Lifeact-YFP (green), which served as morphological reference labeling. All images were smoothed with a one pixel wide 2D Gaussian filter. Scale bar: $2 \mu \mathrm{m}$. 

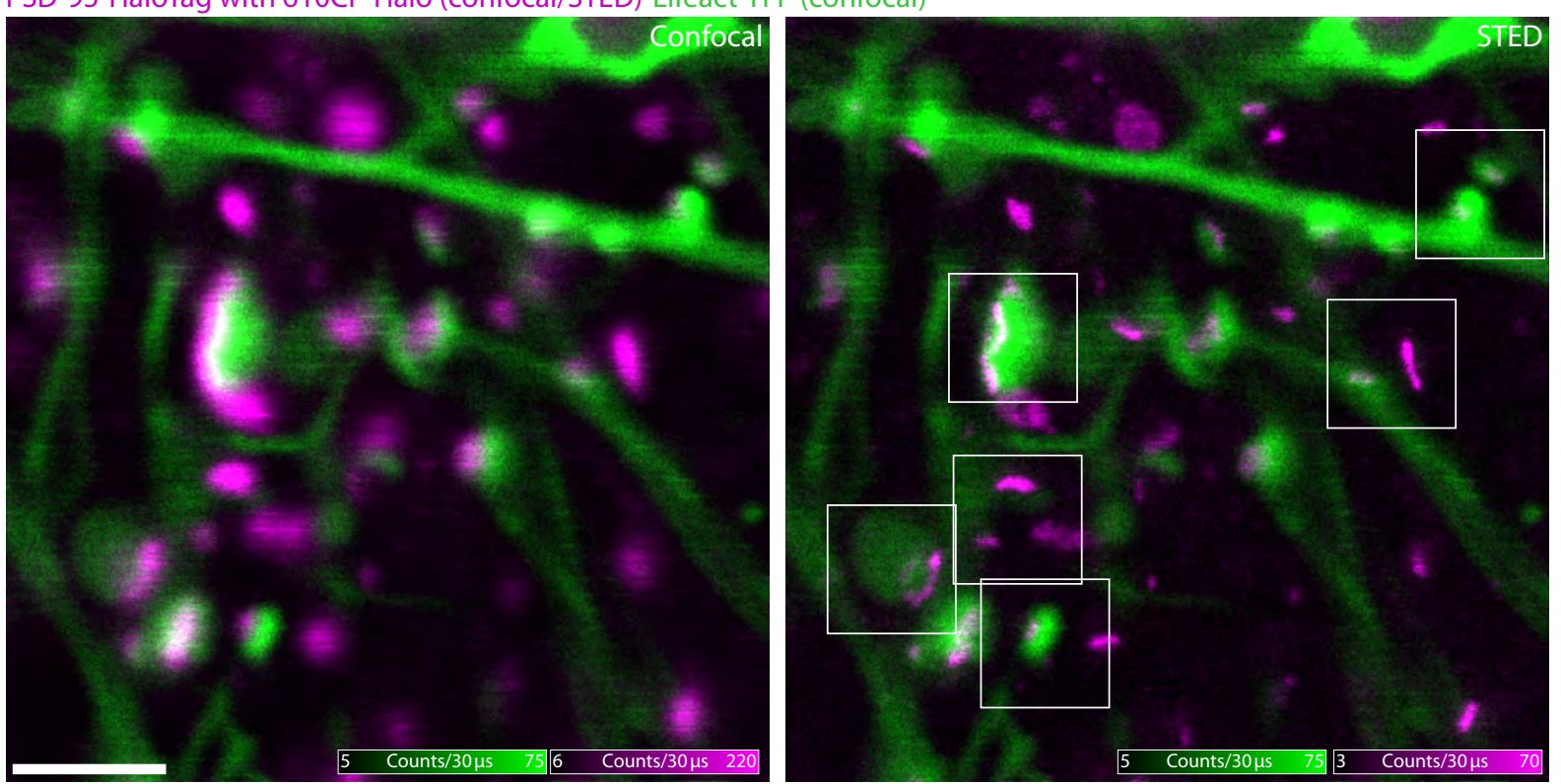

Figure A.11: In vivo STED nanoscopy of endogenous PSD-95 in dendrites in the molecular layer of the visual cortex of a mouse

Confocal (left) and STED (right) images of endogenous PSD-95-HaloTag (magenta) in cortical dendrites, stained with $750 \mathrm{nM} 610 \mathrm{CP}$-Halo-ligand. The STED and the confocal images were overlaid the same confocal image of Lifeact-YFP (green), which served as morphological reference labeling. All images were smoothed with a one pixel wide 2D Gaussian filter. Scale bar: $2 \mu \mathrm{m}$.

PSD-95-HaloTag with 610CP-Halo (confocal/STED) Lifeact-YFP (confocal)

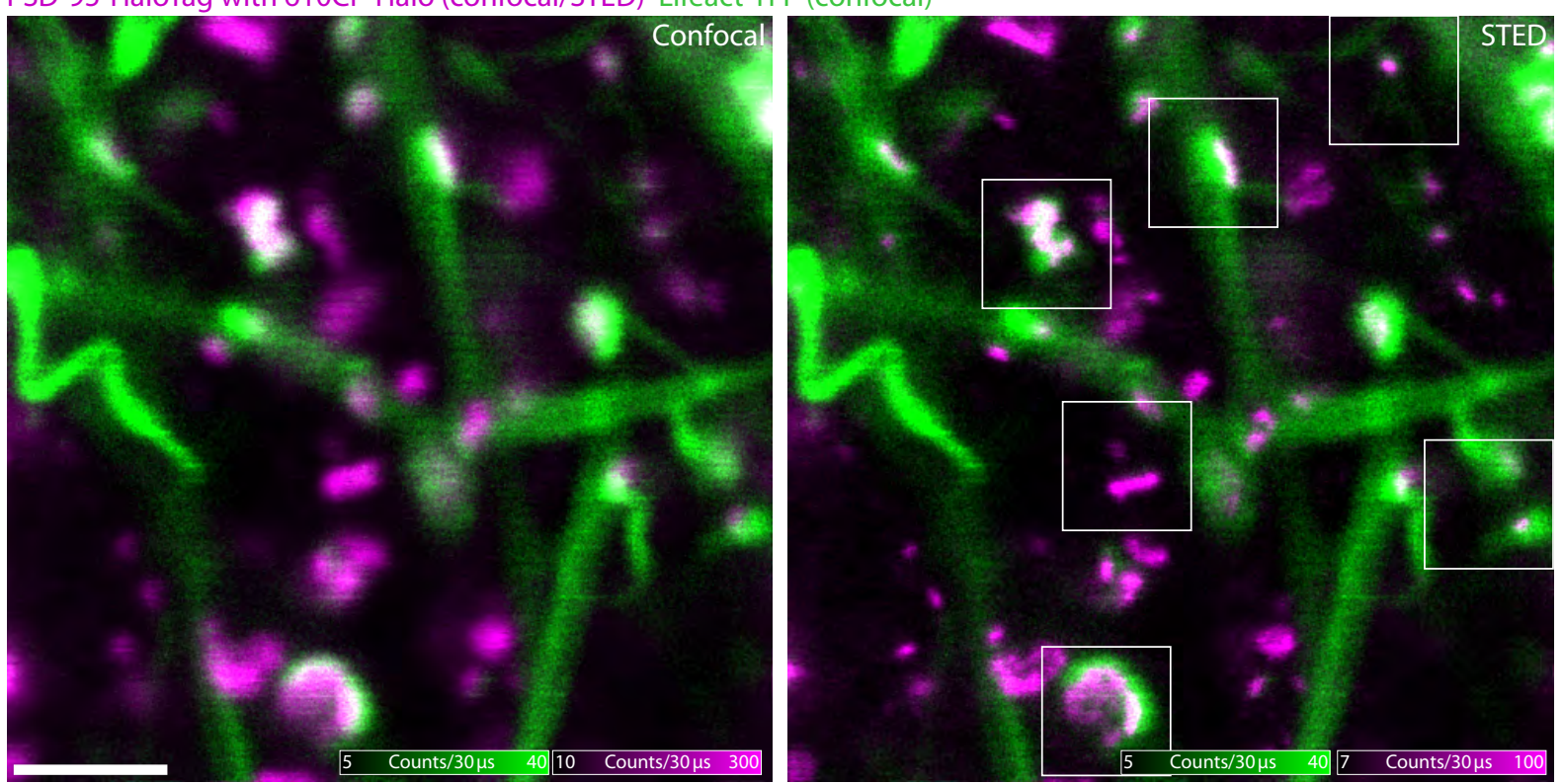

Figure A.12: In vivo STED nanoscopy of endogenous PSD-95 in dendrites in the molecular layer of the visual cortex of a mouse

Confocal (left) and STED (right) images of endogenous PSD-95-HaloTag (magenta) in cortical dendrites, stained with $750 \mathrm{nM} 610 \mathrm{CP}$-Halo-ligand. The STED and the confocal images were overlaid the same confocal image of Lifeact-YFP (green), which served as morphological reference labeling. All images were smoothed with a one pixel wide 2D Gaussian filter. Scale bar: $2 \mu \mathrm{m}$. 


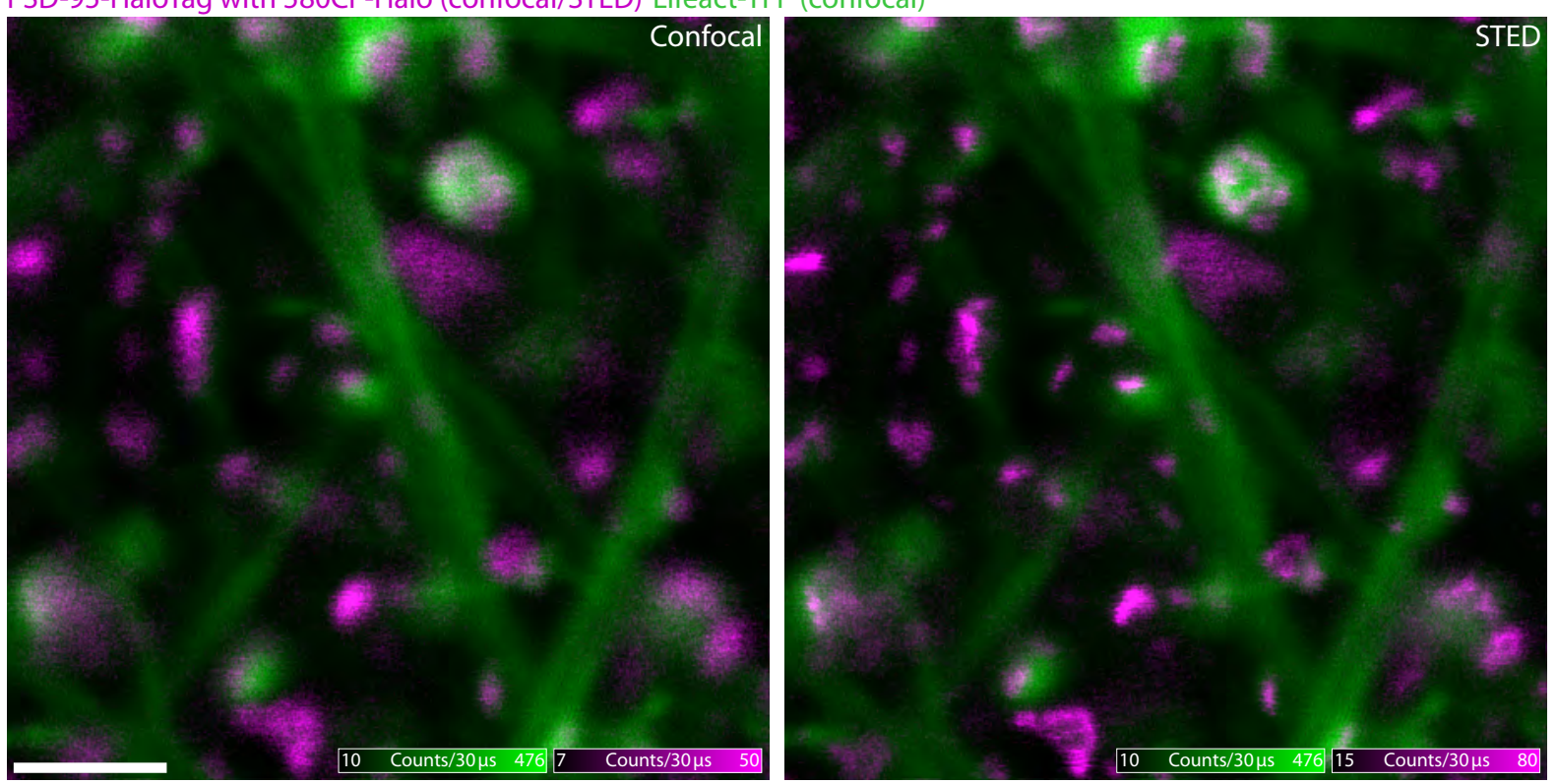

Figure A.13: In vivo STED nanoscopy of endogenous PSD-95 in dendrites in the molecular layer of the visual cortex of a mouse

Confocal (left) and STED (right) images of endogenous PSD-95-HaloTag (magenta) in cortical dendrites, stained with $750 \mathrm{nM} 580 \mathrm{CP}$-Halo-ligand. The STED and the confocal images were overlaid the same confocal image of Lifeact-YFP (green), which served as morphological reference labeling. All images were smoothed with a one pixel wide 2D Gaussian filter. Scale bar: $2 \mu \mathrm{m}$. 


\section{A.5 Imaging parameters}

The following table lists in detail the acquisition parameters of all acquired images within this thesis.

Table A.1: Overview of image acquisition parameters

\begin{tabular}{|c|c|c|c|c|c|c|c|c|c|}
\hline Figure & $\begin{array}{c}\text { Pixel size } \\
\text { in } x / y / z \\
\text { direction } \\
\mathrm{nm} / \mathrm{nm} / \mathrm{nm}\end{array}$ & $\begin{array}{c}\begin{array}{c}\text { Field-of- } \\
\text { view }\end{array} \\
\mu \mathrm{m} \times \mu \mathrm{m}\end{array}$ & $\begin{array}{c}\text { Dwell } \\
\text { time } \\
\mu \mathrm{s}\end{array}$ & $\begin{array}{c}\text { Fluorescent } \\
\text { label }\end{array}$ & $\begin{array}{c}\text { Excitation } \\
\text { power }{ }^{45} \\
\text { (wavelength) } \\
\mu \mathrm{W}(\mathrm{nm})\end{array}$ & $\begin{array}{c}\begin{array}{c}\text { STED } \\
\text { power } \\
45 \\
\mathrm{~mW}\end{array} \\
\end{array}$ & $\begin{array}{c}\begin{array}{c}\text { Detection } \\
\text { channel }\end{array} \\
\mathrm{nm}\end{array}$ & Gating & $\begin{array}{l}\text { Imaging } \\
\text { mode }^{46}\end{array}$ \\
\hline Figure 3.2 & $\begin{array}{l}20 / 20 / 0 \\
20 / 20 / 0 \\
20 / 20 / 0\end{array}$ & $\begin{array}{l}\text { Insets } \\
\text { from } \\
10 \times 10\end{array}$ & $\begin{array}{l}30 \\
30 \\
30\end{array}$ & $\begin{array}{l}\text { crimson } \\
\text { crimson } \\
\text { crimson }\end{array}$ & $\begin{array}{l}5(640) \\
5(640) \\
5(640)\end{array}$ & $\begin{array}{c}- \\
177 \\
177\end{array}$ & $\begin{array}{l}685 \pm 35 \\
685 \pm 35 \\
685 \pm 35\end{array}$ & $\begin{array}{l}\text { yes } \\
\text { yes } \\
\text { no }\end{array}$ & $\begin{array}{l}\text { frame-by- } \\
\text { frame }\end{array}$ \\
\hline Figure 3.3 & $\begin{array}{l}20 / 20 / 100 \\
20 / 20 / 100 \\
20 / 20 / 100\end{array}$ & $\begin{array}{l}9.2 \times 9.2 \\
10 \times 10 \\
10 \times 10\end{array}$ & $\begin{array}{l}20 \\
20 \\
10\end{array}$ & $\begin{array}{l}\text { yellow-green } \\
\text { crimson } \\
\text { crimson }\end{array}$ & $\begin{array}{l}30(473) \\
39(594) \\
29(640)\end{array}$ & $\begin{array}{l}- \\
- \\
-\end{array}$ & $\begin{array}{l}525 \pm 25 \\
623 \pm 12 \\
685 \pm 35\end{array}$ & $\begin{array}{l}\text { no } \\
\text { yes } \\
\text { yes }\end{array}$ & N/A \\
\hline Figure $3.4 a$ & $10 / 10 / 0$ & $5.2 \times 5.2$ & $3 \times 10^{47}$ & crimson & $29(640)$ & 0 & $685 \pm 35$ & yes & \multirow{2}{*}{$\begin{array}{l}\text { frame-by- } \\
\text { frame }\end{array}$} \\
\hline Figure $3.4 \mathrm{~b}$ & $10 / 10 / 0$ & $5.2 \times 5.2$ & $3 \times 10^{47}$ & crimson & $29(640)$ & 177 & $685 \pm 35$ & yes & \\
\hline Figure $3.5 \mathrm{a}$ & $10 / 10 / 0$ & $\begin{array}{c}\text { Inset from } \\
15 \times 15\end{array}$ & 30 & crimson & $29(640)$ & 0 & $685 \pm 35$ & yes & \multirow{4}{*}{$\begin{array}{l}\text { frame-by- } \\
\text { frame }\end{array}$} \\
\hline Figure $3.5 b$ & $10 / 10 / 0$ & $\begin{array}{c}\text { Inset from } \\
15 \times 15\end{array}$ & 30 & crimson & $29(640)$ & 20 & $685 \pm 35$ & yes & \\
\hline Figure $3.5 \mathrm{c}$ & $10 / 10 / 0$ & $\begin{array}{c}\text { Inset from } \\
15 \times 15\end{array}$ & 30 & crimson & $29(640)$ & 66 & $685 \pm 35$ & yes & \\
\hline Figure $3.5 \mathrm{~d}$ & $10 / 10 / 0$ & $\begin{array}{c}\text { Inset from } \\
15 \times 15\end{array}$ & 30 & crimson & $29(640)$ & 177 & $685 \pm 35$ & yes & \\
\hline Figure 3.6a & $20 / 20 / 0$ & $\begin{array}{c}\text { Inset from } \\
18.8 \times 16.8\end{array}$ & 20 & SiR-Halo & $5(640)$ & 0 & $685 \pm 35$ & yes & \multirow{4}{*}{$\begin{array}{l}\text { frame-by- } \\
\text { frame }\end{array}$} \\
\hline Figure $3.6 b$ & $20 / 20 / 0$ & $\begin{array}{c}\text { Inset from } \\
18.2 \times 12.9\end{array}$ & 20 & SiR-Halo & $5(640)$ & 20 & $685 \pm 35$ & yes & \\
\hline Figure $3.6 \mathrm{c}$ & $20 / 20 / 0$ & $\begin{array}{c}\text { Inset from } \\
12.3 \times 13.8\end{array}$ & 20 & SiR-Halo & $5(640)$ & 66 & $685 \pm 35$ & yes & \\
\hline Figure $3.6 \mathrm{~d}$ & $20 / 20 / 0$ & $\begin{array}{c}\text { Inset from } \\
17.4 \times 19.0\end{array}$ & 20 & SiR-Halo & $17(640)$ & 177 & $685 \pm 35$ & yes & \\
\hline Figure $3.8 \mathrm{Al}$ & $20 / 20 / 0$ & $\begin{array}{c}\text { Inset from } \\
6.3 \times 18.4\end{array}$ & 20 & mCardinal & $13(594)$ & 0 & $690 / 70$ & yes & \multirow{2}{*}{$\begin{array}{l}\text { frame-by- } \\
\text { frame } \\
\text { (STED first) }\end{array}$} \\
\hline Figure 3.8All & $20 / 20 / 0$ & $\begin{array}{c}\text { Inset from } \\
6.3 \times 18.4\end{array}$ & 20 & mCardinal & $13(594)$ & 39 & $690 / 70$ & yes & \\
\hline Figure 3.8AIII & $25 / 25 / 0$ & $\begin{array}{c}\text { Inset from } \\
15 \times 16.8\end{array}$ & 20 & mCardinal & $5(594)$ & 0 & $690 / 70$ & yes & \multirow{2}{*}{$\begin{array}{l}\text { frame-by- } \\
\text { frame } \\
\text { (STED first) }\end{array}$} \\
\hline Figure 3.8AIV & $25 / 25 / 0$ & $\begin{array}{c}\text { Inset from } \\
15 \times 16.8\end{array}$ & 20 & mCardinal & $5(594)$ & 53 & $690 / 70$ & yes & \\
\hline Figure $3.8 \mathrm{BI}$ & $25 / 25 / 0$ & $\begin{array}{c}\text { Inset from } \\
18.6 \times 18.8\end{array}$ & 10 & mNeptune2 & $22(594)$ & 0 & $690 / 70$ & yes & \multirow{2}{*}{$\begin{array}{l}\text { frame-by- } \\
\text { frame } \\
\text { (STED first) }\end{array}$} \\
\hline Figure 3.8BII & $25 / 25 / 0$ & $\begin{array}{c}\text { Inset from } \\
18.6 \times 18.8\end{array}$ & 20 & mNeptune2 & $22(594)$ & 66 & $690 / 70$ & yes & \\
\hline Figure $3.8 \mathrm{BIII}$ & $20 / 20 / 0$ & $\begin{array}{c}\text { Inset from } \\
23.5 \times 23.5\end{array}$ & 20 & mNeptune2 & $22(594)$ & 0 & $690 / 70$ & yes & \multirow{2}{*}{$\begin{array}{l}\text { frame-by- } \\
\text { frame } \\
\text { (STED first) }\end{array}$} \\
\hline Figure 3.8BIV & $20 / 20 / 0$ & $23.5 \times 23.5$ & 30 & mNeptune2 & $22(594)$ & 66 & $690 / 70$ & yes & \\
\hline Figure $3.8 \mathrm{Cl}$ & $20 / 20 / 0$ & $5.0 \times 8.9$ & 20 & tagRFP657 & $22(594)$ & 0 & $690 / 70$ & yes & \multirow{2}{*}{$\begin{array}{l}\text { frame-by- } \\
\text { frame } \\
\text { (STED first) }\end{array}$} \\
\hline Figure $3.8 \mathrm{CII}$ & $20 / 20 / 0$ & $5.0 \times 8.9$ & 20 & tagRFP657 & $22(594)$ & 20 & $690 / 70$ & yes & \\
\hline
\end{tabular}

\footnotetext{
${ }^{45}$ All given laser powers refer to the power entering the back aperture of the objective lens. In our custombuilt STED nanoscope, the back aperture of the objective lens was slightly over-illuminated. We measured the laser power before entering the objective lens by using a pinhole with a diameter of $8.25 \mathrm{~mm}$, which corresponds to the diameter of the aperture of the objective lens, in front of the power detector. According to specifications by Leica Microsystems, approximately $88 \%$ of the excitation and $90 \%$ of the depletion laser power entering the objective's back aperture are focused into the sample.

${ }^{46}$ Frame-by-frame: The images were recorded immediately after each other; line-by-line: Quasi-simultaneous acquisition of confocal and STED images was enabled by recording one line of the image in the STED mode followed by an acquisition in the confocal mode of the same line. Images were acquired in the order as they are listed.
}

${ }^{47}$ Each line of the frame was scanned three times and the counts were accumulated. 


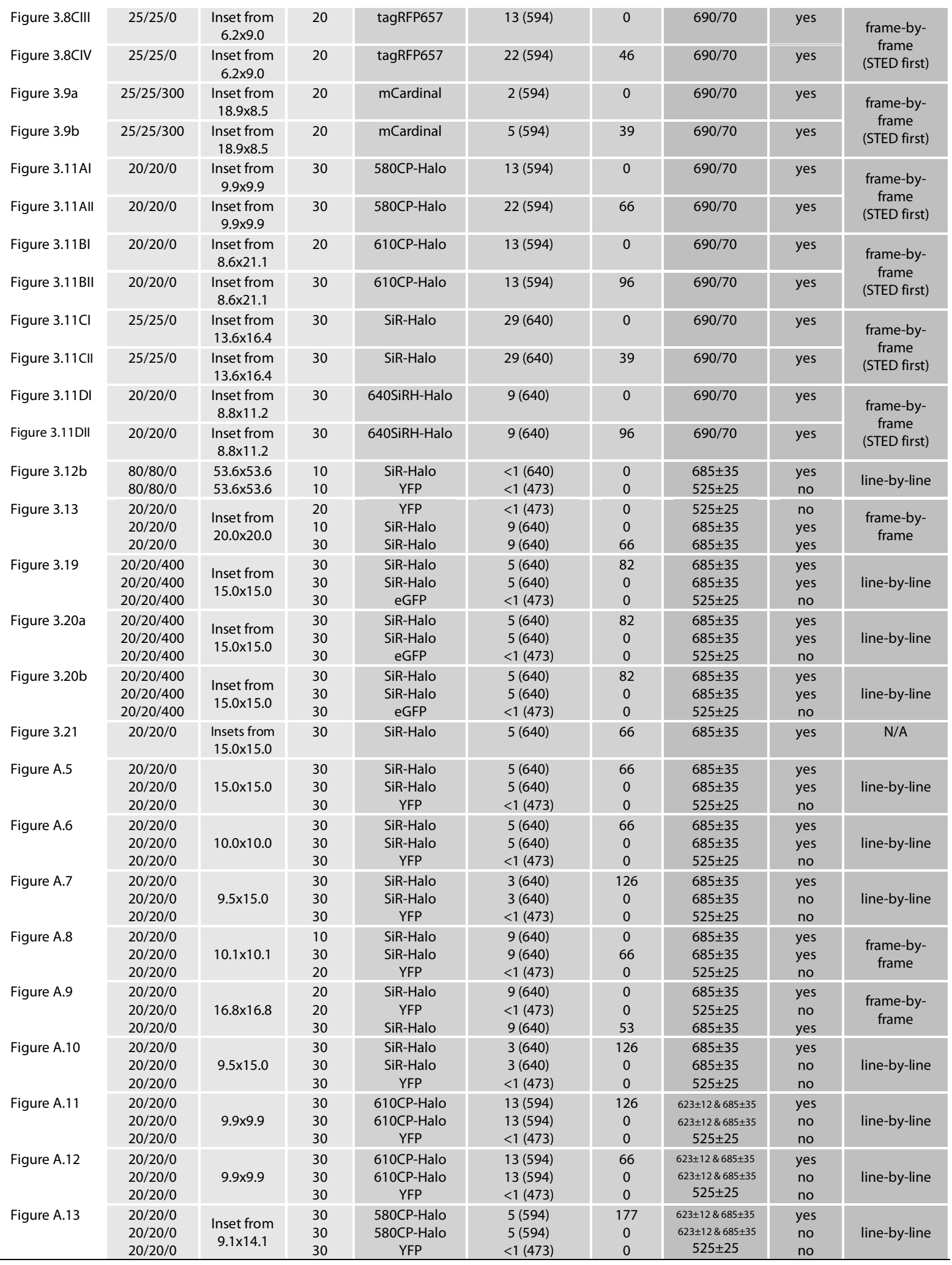




\title{
A.6 Plasmid sequences
}

\section{pAAV1/2-hSyn-Actin-Chromobody-mNeptune2}

\author{
: restriction sites \\ : Actin-Chromobody
}

: mNeptune2

cctgcaggcagctgcgcgctcgctcgctcactgaggccgcccgggcaaagcccgggcgtcgggcgacctttggtcgcccggcctcagtgagcgagcgagcgcgcagagagggagtgg ccaactccatcactaggggttcctgcggccgcacgcgtgtgtctagactgcagagggccctgcgtatgagtgcaagtgggttttaggaccaggatgaggcggggtgggggtgcctacctg acgaccgaccccgacccactggacaagcacccaacccccattccccaaattgcgcatcccctatcagagagggggaggggaaacaggatgcggcgaggcgcgtgcgcactgccagcttc agcaccgcggacagtgccttcgcccccgcctggcggcgcgcgccaccgccgcctcagcactgaaggcgcgctgacgtcactcgccggtcccccgcaaactccccttcccggccaccttggt cgcgtccgcgccgccgccggcccagccggaccgcaccacgcgaggcgcgagataggggggcacgggcgcgaccatctgcgctgcggcgccggcgactcagcgctgcctcagtctgc ggtgggcagcggaggagtcgtgtcgtgcctgagagcgcagtcgagaaggtaccGGATCCATGGCTCAGGTGCAGCTGGTGGAGTCTGGGGGAGGACTGA CGCAGGCAGGGGGCTCTCTGAGACTCTCCTGTGCAACCTCTGGACTAATCTTCAGTGCCTTTGGCATGGGCTGGTTCCGCCAGGCT CCAGGGAAGGAGCGTGAGTTGTAGGAGGTATTAACTGGAGGGGTAGTACAAACTATGGAGACTTCGTGAAGGGCCGATTCACC ATCTCCAGAGACAACGCCAAGAACACGGTGTATTTGCAGATGAACAACCTGAAACCTGAGGACACGGCCGTTIATTACTGCGCAG CACGTATGGTTCACAAAACCGAGTATGACTATTGGGGCGAGGGGACCCAGGTCACCGTCTCCTCAAGAGGTAGTGGTGCCACCG GTGGTACCCCGGGTCGACCTGCAGCCACTAGTGGTAGTGGTGCCATGGTGTCTAAGGGCGAAGAGCTGATTAAGGAGAACATGC ACATGAAGCTGTACATGGAGGGCACCGTGAACAACCACCACTTCAAGTGCACATCCGAGGGCGAAGGCAAGCCCTACGAGGGCA CCCAGACCGGCAGAATCAAGGTGGTCGAGGGCGGCCCTCTCCCCTTCGCCTTCGACATCCTGGCTACCTGCTTCATGTACGGCAG CAAGACCTTCATCAACCACACCCAGGGCATCCCCGATTTCTTAAGCAGTCCTTCCCTGAGGGCTTCACATGGGAGAGAGTCACCA CATACGAAGACGGGGGCGTGCTTACCGTTACCCAGGACACCAGCCTCCAGGACGGCTGCTTGATCTACAACGTCAAGCTCAGAG GGGTGAACTTCCCATCCAACGGCCCTGTGATGCAGAAGAAAACACTCGGCTGGGAGGCCAGTACCGAGACCCTGTACCCCGCTG ACGGCGGCCTGGAAGGCAGATGCGACATGGCCCTGAAGCTCGTGGGCGGGGGCCACCTGCACTGCAACCTGAAGACCACATAC AGATCCAAGAAACCCGCTAAGAACCTCAAGATGCCCGGCGTCTACTITGTGGACCGCAGACTGGAAAGAATCAAGGAGGCCGAC AATGAGACCTACGTCGAGCAGCACGAGGTGGCTGTGGCCAGATACTGCGACCTCCCTAGCAAACTGGGGCACAAACTTAATGGC ATGGACGAGCTGTACAAGTAAGAATTCgatatcaagcttatcgataatcaacctctggattacaaaatttgtgaaagattgactggtattcttaactatgttgctccttttacgcta tgtggatacgctgctttaatgcctttgtatcatgctattgcttcccgtatggctttcattttctcctccttgtataaatcctggttgctgtctctttatgaggagttgtggcccgttgtcaggcaacgtg gcgtggtgtgcactgtgtttgctgacgcaacccccactggttggggcattgccaccacctgtcagctcctttccgggactttcgctttccccetccetattgccacggcggaactcatcgccgcct gccttgcccgctgctggacaggggctcggctgttgggcactgacaattccgtggtgttgtcggggaaatcatcgtcctttccttggctgctcgcctgtgttgccacctggattctgcgcgggac gtccttctgctacgtccettcggccctcaatccagcggaccttccttcccgcggectgctgccggctctgcggcctcttccgcgtcttcgccttcgccctcagacgagtcggatctccctttgggc cgcctccccgcatcgataccgagcgctgctcgagagatctacgggtggcatccctgtgacccctccccagtgcctctcctggecctggaagttgccactccagtgeccaccagccttgtcctaa taaaattaagttgcatcattttgtctgactaggtgtccttctataatattatggggtggaggggggtggtatggagcaaggggcaagttgggaagacaacctgtagggectgcggggtctatt gggaaccaagctggagtgcagtggcacaatcttggctcactgcaatctccgcctcctgggttcaagcgattctcctgcctcagcctcccgagttgttgggattccaggcatgcatgaccaggc tcagctaatttttgttttttggtagagacggggtttcaccatattggccaggctggtctccaactcctaatctcaggtgatctacccaccttggcctcccaaattgctgggattacaggcgtgaac cactgctcccttccctgtccttctgattttgtaggtaaccacgtgcggaccgagcggccgcaggaacccctagtgatggagttggccactccctctctgcgcgctcgctcgctcactgaggccg ggcgaccaaaggtcgcccgacgcccgggctttgcccggg g gectcagtgagcgagcgagcgcgcagctgcctgcaggggcgcctgatgcggtattttctccttacgcatctgtgcggta tttcacaccgcatacgtcaaagcaaccatagtacgcgccetgtagcggcgcattaagcgcggcgggtgtggtggttacgcgcagcgtgaccgctacacttgccagcgccctagcgcccgct cctttcgctttcttcccttcctttctcgccacgttcgccggctttccccgtcaagctctaaatcgggggctccctttagggttccgatttagtgctttacggcacctcgaccccaaaaaacttgatttg ggtgatggttcacgtagtgggccatcgccctgatagacggtttttcgccctttgacgttggagtccacgttctttaatagtggactcttgttccaaactggaacaacactcaaccctatctcgggc tattcttttgatttataagggattttgccgatttcggcctattggttaaaaaatgagctgatttaacaaaaatttaacgcgaattttaacaaaatattaacgtttacaattttatggtgcactctcagtac aatctgctctgatgccgcatagttaagccagccccgacacccgccaacacccgctgacgcgccctgacgggcttgtctgctcccggcatccgcttacagacaagctgtgaccgtctccggga gctgcatgtgtcagaggttttcaccgtcatcaccgaaacgcgcgagacgaaagggcctcgtgatacgcctatttttataggttaatgtcatgataataatggtttcttagacgtcaggtggcact tttcggggaaatgtgcgcggaacccctatttgtttattttctaaatacattcaaatatgtatccgctcatgagacaataaccctgataaatgcttcaataatattgaaaaaggaagagtatgagtat tcaacatttccgtgtcgcccttattccctttttgcggcattttgccttcctgtttttgctcacccagaaacgctggtgaaagtaaaagatgctgaagatcagttgggtgcacgagtgggttacatcg aactggatctcaacagcggtaagatccttgagagttttcgccccgaagaacgttttccaatgatgagcacttttaaagttctgctatgtggcgcggtattatcccgtattgacgccgggcaagag caactcggtcgccgcatacactattctcagaatgacttggttgagtactcaccagtcacagaaaagcatcttacggatggcatgacagtaagagaattatgcagtgctgccataaccatgagtg ataacactgcggccaacttacttctgacaacgatcggaggaccgaaggagctaaccgctttttgcacaacatgggggatcatgtaactcgccttgatcgttgggaaccggagctgaatgaa gccataccaaacgacgagcgtgacaccacgatgcctgtagcaatggcaacaacgttgcgcaaactattaactggcgaactacttactctagcttcccggcaacaattaatagactggatggag gcggataaagttgcaggaccacttctgcgctcggcccttccggctggctggtttattgctgataaatctggagccggtgagcgtgggtctcgcggtatcattgcagcactggggccagatgg taagccctcccgtatcgtagttatctacacgacggggagtcaggcaactatggatgaacgaaatagacagatcgctgagataggtgcctcactgattaagcattggtaactgtcagaccaagt ttactcatatatactttagattgatttaaaacttcatttttaatttaaaaggatctaggtgaagatcctttttgataatctcatgaccaaaatcccttaacgtgagttttcgttccactgagcgtcagacc ccgtagaaaagatcaaaggatcttcttgagatccttttttctgcgcgtaatctgctgcttgcaaacaaaaaaaccaccgctaccagcggtggtttgtttgccggatcaagagctaccaactctttt tccgaaggtaactggcttcagcagagcgcagataccaaatactgtccttctagtgtagccgtagttaggccaccacttcaagaactctgtagcaccgcctacatacctcgctctgctaatcctgtt accagtggctgctgccagtggcgataagtcgtgtcttaccgggttggactcaagacgatagttaccggataaggcgcagcggtcgggctgaacggggggttcgtgcacacagcccagctt ggagcgaacgacctacaccgaactgagatacctacagcgtgagctatgagaaagcgccacgcttcccgaagggagaaaggcggacaggtatccggtaagcggcagggtcggaacagg agagcgcacgagggagcttccagggggaaacgcctggtatctttatagtcctgtcgggtttcgccacctctgacttgagcgtcgattttgtgatgctcgtcaggggggcggagcctatgga aaaacgccagcaacgcggcctttttacggttcctggccttttgctggecttttgctcacatgt 


\section{pAAV1/2-hSyn-Lifeact-tagRFP657}

\section{: restriction sites : : : Lifeact $\quad$ : tagRFP657}

cctgcaggcagctgcgcgctcgctcgctcactgaggccgcccgggcaaagcccgggcgtcgggcgactttggtcgcccggctcagtgagcgagcgagcgcgcagagagggagtgg ccaactccatcactaggggttcctgcggccgcacgcgtgtgtctagactgcagagggcctgcgtatgagtgcaagtgggtttaggaccaggatgaggcggggtgggggtgcctacctg acgaccgaccccgacccactggacaagcacccaacccccattccccaaattgcgcatccctatcagagagggggaggggaaacaggatgcggcgaggcgcgtgcgcactgccagcttc agcaccgcggacagtgccttcgcccccgcctggcggcgcgcgccaccgccgcctcagcactgaaggcgcgctgacgtcactcgccggtcccccgcaaactccccttcccggccaccttggt cgcgtccgcgccgccgccggcccagccggaccgcaccacgcgaggcgcgagataggggggcacgggcgcgaccatctgcgctgcggcgccggcgactcagcgctgcctcagtctgc ggtgggcagcggaggagtcgtgtcgtgcctgagagcgcagtcgagaaggtaccGGATCCAATGGGTGTCGCAGATTTGATCAAGAAATTCGAAAGCATCT CAAAGGAAGAAGGGGACGCACCGGTCGCCACCATGAGCGAGCTGATCACCGAGAACATGCACATGAAGCTGTACATGGAGGGC ACCGTGAACAACCACCACTTCAAGTGCACATCCGAGGGCGAAGGCAAGCCCTACGAGGGCACCCAGACCCAGAGAATCAAGGT GGTCGAGGGCGGCCCTCTCCCCTTCGCCTTCGACATCCTGGCTACCAGCTTCATGTACGGCAGTCACACCTTCATCAACCACACCC AGGGCATCCCCGACTTCTGGAAGCAGTCCTTCCCTGAGGGCTTCACATGGGAGAGAGTCACCACATACGAAGACGGGGGCGTGC TGACCGCTACCCAGGACACCAGCCTCCAGGACGGCTGCCTCATCTACAACGTCAAGATCAGAGGGGTGAACTTCCCATCCAACGC CCCTGTGATGCAGAAGAAAACACTCGGCTGGGAGGCCCACACCGAGATGCTGTACCCCGCTGACGGCGGCCTGGAAGGCAGAAC CGCGCTGGCCCTGAAGCTCGTGGGCGGGGGCCACCTGATCTGCAACTTCAAGACCACATACAGATCCAAGAAACCCGCTAAGAA CCTCAAGATGCCCGGCGTCTACTATGTGGACTACAGACTGGAAAGAATCAAGGAGGCCGACAAAGAGACCTACGTCGAGCAGCA CGAGGTGGCTGTGGCCAGATACTGCGACCTCCCTAGCAAACTGGGGCACAAGCTTAATTAAGAATTCgatatcaagcttatcgataatcaacctct ggattacaaaatttgtgaaagattgactggtattcttaactatgttgctccttttacgctatgtggatacgctgctttaatgcctttgtatcatgctattgcttcccgtatggctttcattttctcctccttg tataaatcctggttgctgtctctttatgaggagttgtggcccgttgtcaggcaacgtggcgtggtgtgcactgtgttgctgacgcaacccccactggttggggcattgccaccacctgtcagct cctttccgggactttcgctttcccctccctattgccacggcggaactcatcgccgcctgccttgcccgctgctggacaggggctcggctgttgggcactgacaattccgtggtgttgtcgggg aaatcatcgtcctttccttggctgctcgcctgtgttgccacctggattctgcgcgggacgtccttctgctacgtccettcggccctcaatccagcggaccttccttcccgcggcctgctgccggctc tgcggcctcttccgcgtcttcgccttcgccetcagacgagtcggatctccctttgggecgcctccccgcatcgataccgagcgetgctcgagagatctacgggtggcatccctgtgacccetcc ccagtgcctctcctggccctggaagttgccactccagtgcccaccagccttgtcctaataaaattaagttgcatcatttgtctgactaggtgtccttctataatattatggggtggaggggggtg gtatggagcaaggggcaagttgggaagacaacctgtagggcctgcggggtctattgggaaccaagctggagtgcagtggcacaatcttggctcactgcaatctccgcctcctgggttcaa gcgattctcctgcctcagcctcccgagttgttgggattccaggcatgcatgaccaggctcagctaatttttgttttttggtagagacggggtttcaccatattggccaggctggtctccaactcct aatctcaggtgatctacccaccttggcctcccaaattgctgggattacaggcgtgaaccactgctcccttcctgtccttctgattttgtaggtaaccacgtgcggaccgagcggccgcaggaa ccctagtgatggagttggccactccctctctgcgcgctcgctcgctcactgaggccgggcgaccaaaggtcgcccgacgcccgggctttgcccgggcggcctcagtgagcgagcgagcg cgcagctgcctgcaggggcgcctgatgcggtatttctccttacgcatctgtgcggtatttcacaccgcatacgtcaaagcaaccatagtacgcgcctgtagcggcgcattaagcgcggcgg gtgtggtggttacgcgcagcgtgaccgctacacttgccagcgcctagcgcccgctcctttcgctttcttcccttcctttctcgccacgttcgccggctttccccgtcaagctctaaatcgggggc tccctttagggttccgatttagtgctttacggcacctcgaccccaaaaaacttgatttgggtgatggttcacgtagtgggecatcgccctgatagacggtttttcgccctttgacgttggagtcca cgttctttaatagtggactcttgttccaaactggaacaacactcaaccctatctcgggctattcttttgatttataagggattttgccgatttcggcctattggttaaaaaatgagctgatttaacaaa aatttaacgcgaattttaacaaaatattaacgtttacaattttatggtgcactctcagtacaatctgctctgatgccgcatagttaagccagccccgacacccgccaacacccgctgacgcgccct gacgggcttgtctgctcccggcatccgcttacagacaagctgtgaccgtctccgggagctgcatgtgtcagaggttttcaccgtcatcaccgaaacgcgcgagacgaaagggcctcgtgata cgcctattttataggttaatgtcatgataataatggtttcttagacgtcaggtggcacttttcggggaaatgtgcgcggaacccctatttgtttattttctaaatacattcaaatatgtatccgctca tgagacaataaccctgataaatgcttcaataatattgaaaaaggaagagtatgagtattcaacatttccgtgtcgeccttattcccttttttgcggcattttgccttcctgtttttgctcacccagaaac gctggtgaaagtaaaagatgctgaagatcagttgggtgcacgagtgggttacatcgaactggatctcaacagcggtaagatccttgagagttttcgccccgaagaacgttttccaatgatga gcacttttaaagttctgctatgtggcgcggtattatcccgtattgacgccgggcaagagcaactcggtcgccgcatacactattctcagaatgacttggttgagtactcaccagtcacagaaaa gcatcttacggatggcatgacagtaagagaattatgcagtgctgccataaccatgagtgataacactgcggccaacttacttctgacaacgatcggaggaccgaaggagctaaccgctttttt gcacaacatgggggatcatgtaactcgccttgatcgttgggaaccggagctgaatgaagccataccaaacgacgagcgtgacaccacgatgcctgtagcaatggcaacaacgttgcgcaa actattaactggcgaactacttactctagcttcccggcaacaattaatagactggatggaggcggataaagttgcaggaccacttctgcgctcggcccttccggctggctggtttattgctgata aatctggagccggtgagcgtgggtctcgcggtatcattgcagcactggggccagatggtaagccctcccgtatcgtagttatctacacgacggggagtcaggcaactatggatgaacgaaa tagacagatcgctgagataggtgcctcactgattaagcattggtaactgtcagaccaagtttactcatatatactttagattgatttaaaacttcatttttaatttaaaaggatctaggtgaagatcct ttttgataatctcatgaccaaaatcccttaacgtgagttttcgttccactgagcgtcagaccccgtagaaaagatcaaaggatcttcttgagatcctttttttctgcgcgtaatctgctgcttgcaaac aaaaaaaccaccgctaccagcggtggtttgtttgccggatcaagagctaccaactctttttccgaaggtaactggcttcagcagagcgcagataccaaatactgtccttctagtgtagccgtagt taggccaccacttcaagaactctgtagcaccgcctacatacctcgctctgctaatcctgttaccagtggctgctgccagtggcgataagtcgtgtcttaccgggttggactcaagacgatagtta ccggataaggcgcagcggtcgggctgaacggggggttcgtgcacacagcccagcttggagcgaacgacctacaccgaactgagatacctacagcgtgagctatgagaaagcgccacgc ttcccgaagggagaaaggcggacaggtatccggtaagcggcagggtcggaacaggagagcgcacgagggagcttccagggggaaacgcctggtatctttatagtcctgtcgggtttcg ccacctctgacttgagcgtcgatttttgtgatgctcgtcaggggggcggagcctatggaaaaacgccagcaacgcggcctttttacggttcctggccttttgctggccttttgctcacatgt 


\section{pAAV1/2-hSyn-Lifeact-mGarnet}

\section{: restriction sites : : Lifeact : mGarnet}

cctgcaggcagctgcgcgctcgctcgctcactgaggccgcccgggcaaagcccgggcgtcgggcgacctttggtcgcccggctcagtgagcgagcgagcgcgcagagagggagtgg ccaactccatcactaggggttcctgcggccgcacgcgtgtgtctagactgcagagggcctgcgtatgagtgcaagtgggtttaggaccaggatgaggcggggtgggggtgcctacctg acgaccgaccccgacccactggacaagcacccaacccccattccccaaattgcgcatccctatcagagagggggaggggaaacaggatgcggcgaggcgcgtgcgcactgccagcttc agcaccgcggacagtgccttcgcccccgcctggcggcgcgcgccaccgccgcctcagcactgaaggcgcgetgacgtcactcgccggtcccccgcaaactccccttcccggccaccttggt cgcgtccgcgccgccgccggcccagccggaccgcaccacgcgaggcgcgagataggggggcacgggcgcgaccatctgcgctgcggcgccggcgactcagcgctgcctcagtctgc ggtgggcagcggaggagtcgtgtcgtgcctgagagcgcagtcgagaaggtaccGGATCCAATGGGTGTCGCAGATTTGATCAAGAAATTCGAAACATCT CAAAGGAAGAAGGGGACGCACCGGGAATGAACAGCCTGATCAAAGAAAACATGCGGATGAAGGTGGTGCTGGAAGGCAGCGT GAACGGCCACCAGTTCAAGTGCACCGGCGAGGGCGAGGGCAACCCCTACATGGGCACCCAGACCATGCGGATCAAAGTGATCG AGGGCGGACCTCTGCCCTTCGCCTTCGACATCCTGGCCACATCCTTCATGTACGGCAGCAAGACCTTCATCAAGTACCCCAAGGGC ATCCCCGATTCTTCAAGCAGAGCTTCCCCGAGGGCTTCACCTGGGAGAGAGTGACCAGATACGAGGACGGCGGCGTGATCACC GTGATGCAGGACACCAGCCTGGAAGATGGCTGCCTGGTGTACCATGCCCAGGTCAGGGGCGTGAATITCCCAGCAACGGCGCC GTGATGCAGAAGAAAACCAAGGGCTGGGAGCCCAACACCGAGATGATGTACCCCGCTGACGGCGGACTGAGAGGCTACAACCA CATGGCCCTGAAGGTGGACGGCGGAGGGCACCTGAGCTGCAGCCTGGTGACCACCTACCGGTCCAAGAAAACCGTGGGCAACAT CAAGATGCCCGGCATCCACGCCGTGGACCGGCGGCTGGAAAGGCTGGAAGAGTCCGACAACGAGATGTTCGTGGTGCAGCGGG AGCACGCCGTGGCCAAGTTCGCCGGCCTGGGCCGGAGGGTGAAAATCTAGGAATTCgatatcaagcttatcgataatcaacctctggattacaaaattt gtgaaagattgactggtattcttaactatgttgctccttttacgctatgtggatacgctgctttaatgcctttgtatcatgctattgcttcccgtatggctttcattttctcctccttgtataaatcctggtt gctgtctctttatgaggagttgtggcccgttgtcaggcaacgtggcgtggtgtgcactgtgtttgctgacgcaacccccactggttggggcattgccaccacctgtcagctcctttccgggactt tcgctttccccctccctattgccacggcggaactcatcgccgctgcttgcccgctgctggacaggggetcggetgttgggcactgacaattccgtggtgttgtcggggaaatcatcgtccttt ccttggctgctcgcctgtgttgccacctggattctgcgcgggacgtccttctgctacgtccettcggccctcaatccagcggaccttccttcccgcggcctgctgccggctctgcggectcttccg cgtcttcgccttcgecctcagacgagtcggatctccctttgggccgcctccccgcatcgataccgagcgetgctcgagagatctacgggtggcatccctgtgacccctccccagtgcctctcct ggccctggaagttgccactccagtgcccaccagccttgtcctaataaaattaagttgcatcattttgtctgactaggtgtccttctataatattatggggtggaggggggtggtatggagcaag gggcaagttgggaagacaacctgtagggcctgcggggtctattgggaaccaagctggagtgcagtggcacaatcttggctcactgcaatctccgcctcctgggttcaagcgattctcctgcc tcagcctcccgagttgttgggattccaggcatgcatgaccaggctcagctaattttgttttttggtagagacggggtttcaccatattggccaggctggtctccaactcctaatctcaggtgatc tacccaccttggcctcccaaattgctgggattacaggcgtgaaccactgctccttccetgtccttctgattttgtaggtaaccacgtgcggaccgagcggccgcaggaaccctagtgatgga gttggccactccctctctgcgcgctcgctcgctcactgaggccgggcgaccaaaggtcgcccgacgcccgggctttgcccgggcggcctcagtgagcgagcgagcgcgcagctgcctgc aggggcgcctgatgcggtatttctccttacgcatctgtgcggtatttcacaccgcatacgtcaaagcaaccatagtacgcgcctgtagcggcgcattaagcgcggcgggtgtggtggttac gcgcagcgtgaccgctacacttgccagcgccctagcgcccgctcctttcgctttcttcccttcctttctcgccacgttcgccggctttccccgtcaagctctaaatcgggggctccctttagggttc cgatttagtgctttacggcacctcgaccccaaaaaacttgatttgggtgatggttcacgtagtgggccatcgccctgatagacggtttttcgccctttgacgttggagtccacgttctttaatagtg gactcttgttccaaactggaacaacactcaacctatctcgggctattctttgatttataagggattttgccgatttcggcctattggttaaaaaatgagctgatttaacaaaaatttaacgcgaatt ttaacaaaatattaacgtttacaattttatggtgcactctcagtacaatctgctctgatgccgcatagttaagccagccccgacacccgccaacacccgctgacgcgccctgacgggcttgtctgc tcccggcatccgcttacagacaagctgtgaccgtctccgggagctgcatgtgtcagaggttttcaccgtcatcaccgaaacgcgcgagacgaaagggcctcgtgatacgcctattttatagg ttaatgtcatgataataatggtttcttagacgtcaggtggcacttttcggggaaatgtgcgcggaacccctatttgtttatttttctaaatacattcaaatatgtatccgctcatgagacaataaccct gataaatgcttcaataatattgaaaaaggaagagtatgagtattcaacatttccgtgtcgcccttattccctttttgcggcattttgccttcctgtttttgctcacccagaaacgctggtgaaagtaa aagatgctgaagatcagttgggtgcacgagtgggttacatcgaactggatctcaacagcggtaagatccttgagagttttcgccccgaagaacgtttccaatgatgagcacttttaaagttct gctatgtggcgcggtattatcccgtattgacgccgggcaagagcaactcggtcgccgcatacactattctcagaatgacttggttgagtactcaccagtcacagaaaagcatcttacggatgg catgacagtaagagaattatgcagtgctgccataaccatgagtgataacactgcggccaacttacttctgacaacgatcggaggaccgaaggagctaaccgctttttgcacaacatggggg atcatgtaactcgccttgatcgttgggaaccggagctgaatgaagccataccaaacgacgagcgtgacaccacgatgcctgtagcaatggcaacaacgttgcgcaaactattaactggcgaa ctacttactctagcttcccggcaacaattaatagactggatggaggcggataaagttgcaggaccacttctgcgctcggcccttccggctggctggtttattgctgataaatctggagccggtg agcgtgggtctcgcggtatcattgcagcactggggccagatggtaagccctcccgtatcgtagttatctacacgacggggagtcaggcaactatggatgaacgaaatagacagatcgctga gataggtgcctcactgattaagcattggtaactgtcagaccaagtttactcatatatactttagattgatttaaaacttcatttttaatttaaaaggatctaggtgaagatcctttttgataatctcatg accaaaatcccttaacgtgagttttcgttccactgagcgtcagaccccgtagaaaagatcaaaggatcttcttgagatcctttttttctgcgcgtaatctgctgcttgcaaacaaaaaaaccaccgc taccagcggtggtttgtttgccggatcaagagctaccaactcttttccgaaggtaactggcttcagcagagcgcagataccaaatactgtccttctagtgtagccgtagttaggccaccacttc aagaactctgtagcaccgcctacatacctcgctctgctaatcctgttaccagtggctgctgccagtggcgataagtcgtgtcttaccgggttggactcaagacgatagttaccggataaggcgc agcggtcgggctgaacggggggttcgtgcacacagcccagcttggagcgaacgacctacaccgaactgagatacctacagcgtgagctatgagaaagcgccacgcttcccgaagggag aaaggcggacaggtatccggtaagcggcagggtcggaacaggagagcgcacgagggagcttccagggggaaacgcctggtatctttatagtcctgtcgggtttcgccacctctgacttga gcgtcgattttgtgatgctcgtcaggggggcggagcctatggaaaaacgccagcaacgcggccttttacggttcctggccttttgctggccttttgctcacatgt 


\section{pAAV1/2-hSyn-eGFP}

: restriction sites

cctgcaggcagctgcgcgctcgctcgctcactgaggccgcccgggcaaagcccgggcgtcgggcgacctttggtcgcccggcctcagtgagcgagcgagcgcgcagagagggagtgg ccaactccatcactaggggttcctgcggccgcacgcgtgtgtctagactgcagagggcctgcgtatgagtgcaagtgggtttaggaccaggatgaggcggggtgggggtgcctacctg acgaccgaccccgacccactggacaagcacccaacccccattccccaaattgcgcatcccctatcagagagggggaggggaaacaggatgcggcgaggcgcgtgcgcactgccagcttc agcaccgcggacagtgccttcgcccccgcctggcggcgcgcgccaccgccgcctcagcactgaaggcgcgctgacgtcactcgccggtcccccgcaaactccccttcccggccaccttggt cgcgtccgcgccgccgccggcccagccggaccgcaccacgcgaggcgcgagataggggggcacgggegcgaccatctgcgctgcggcgccggcgactcagcgctgcctcagtctgc ggtgggcagcggaggagtcgtgtcgtgcctgagagcgcagtcgagaaggtaccGGATCCGATGGTGAGCAAGGGCGAGGAGCTGTTCACCGGGGTGGT GCCCATCCTGGTCGAGCTGGACGGCGACGTAAACGGCCACAAGTTCAGCGTGTCCGGCGAGGGCGAGGGCGATGCCACCTACG GCAAGCTGACCCTGAAGTTCATCTGCACCACCGGCAAGCTGCCCGTGCCCTGGCCCACCCTCGTGACCACCCTGACCTACGGCGT GCAGTGCTTCAGCCGCTACCCCGACCACATGAAGCAGCACGACTTCTTCAAGTCCGCCATGCCCGAAGGCTACGTCCAGGAGCGC ACCATCTTCTTCAAGGACGACGGCAACTACAAGACCCGCGCCGAGGTGAAGTTCGAGGGCGACACCCTGGTGAACCGCATCGAG CTGAAGGGCATCGACTTCAAGGAGGACGGCAACATCCTGGGGCACAAGCTGGAGTACAACTACAACAGCCACAACGTCTATATC ATGGCCGACAAGCAGAAGAACGGCATCAAGGTGAACTTCAAGATCCGCCACAACATCGAGGACGGCAGCGTGCAGCTCGCCGA CCACTACCAGCAGAACACCCCCATCGGCGACGGCCCCGTGCTGCTGCCCGACAACCACTACCTGAGCACCCAGTCCGCCCTGAGC AAAGACCCCAACGAGAAGCGCGATCACATGGTCCTGCTGGAGTTCGTGACCGCCGCCGGGATCACTCTCGGCATGGACGAGCTG TACAAGTAACTGGAATTCgatatcaagcttatcgataatcaacctctggattacaaaatttgtgaaagattgactggtattcttaactatgttgctccttttacgctatgtggatacgct gctttaatgcctttgtatcatgctattgcttcccgtatggctttcattttctcctccttgtataaatcctggttgctgtctctttatgaggagttgtggcccgttgtcaggcaacgtggcgtggtgtgca ctgtgtttgctgacgcaacccccactggttggggcattgccaccacctgtcagctcctttccgggactttcgctttccccetccctattgccacggcggaactcatcgccgcctgccttgcccgct gctggacaggggctcggctgttgggcactgacaattccgtggtgttgtcggggaaatcatcgtcctttccttggctgctcgcctgtgttgccacctggattctgcgcgggacgtccttctgcta cgtcccttcggccctcaatccagcggaccttccttcccgcggcctgctgccggctctgcggcctcttccgcgtcttcgecttcgccctcagacgagtcggatctccctttgggccgcctccccgc atcgataccgagcgctgctcgagagatctacgggtggcatccetgtgacccctccccagtgcctctcctggccctggaagttgccactccagtgcccaccagccttgtcctaataaaattaagtt gcatcattttgtctgactaggtgtccttctataatattatggggtggaggggggtggtatggagcaaggggcaagttgggaagacaacctgtagggcctgcggggtctattgggaaccaag ctggagtgcagtggcacaatcttggctcactgcaatctccgctcctgggttcaagcgattctcctgcctcagcctcccgagttgttgggattccaggcatgcatgaccaggctcagctaattttt gttttttggtagagacggggtttcaccatattggccaggctggtctccaactcctaatctcaggtgatctacccaccttggcctcccaaattgctgggattacaggcgtgaaccactgctcccttc cctgtccttctgattttgtaggtaaccacgtgcggaccgagcggccgcaggaaccctagtgatggagttggccactccctctctgcgcgctcgctcgctcactgaggccgggcgaccaaag gtcgcccgacgcccgggctttgcccgggcggcctcagtgagcgagcgagcgcgcagctgcctgcaggggcgcctgatgcggtattttctccttacgcatctgtgcggtatttcacaccgcat acgtcaaagcaaccatagtacgcgccctgtagcggcgcattaagcgcggcgggtgtggtggttacgcgcagcgtgaccgctacacttgccagcgccctagcgcccgctcctttcgctttcttc ccttcctttctcgccacgttcgccggcttccccgtcaagctctaaatcgggggctccetttagggttccgatttagtgctttacggcacctcgaccccaaaaaacttgatttgggtgatggttcac gtagtgggccatcgccctgatagacggttttcgccctttgacgttggagtccacgttctttaatagtggactcttgttccaaactggaacaacactcaaccctatctcgggctattcttttgatttat aagggattttgccgatttcggcctattggttaaaaaatgagctgatttaacaaaaatttaacgcgaattttaacaaaatattaacgtttacaattttatggtgcactctcagtacaatctgctctgatg ccgcatagttaagccagccccgacacccgccaacacccgctgacgcgccctgacgggcttgtctgctcccggcatccgcttacagacaagctgtgaccgtctccgggagctgcatgtgtcag aggttttcaccgtcatcaccgaaacgcgcgagacgaaagggcctcgtgatacgcctatttttataggttaatgtcatgataataatggtttcttagacgtcaggtggcacttttcggggaaatgt gcgcggaaccctatttgtttatttttctaaatacattcaaatatgtatccgctcatgagacaataaccctgataaatgcttcaataatattgaaaaaggaagagtatgagtattcaacatttccgtgt cgcccttattccctttttgcggcattttgccttcctgtttttgctcacccagaaacgctggtgaaagtaaaagatgctgaagatcagttgggtgcacgagtgggttacatcgaactggatctcaac agcggtaagatccttgagagttttcgccccgaagaacgttttccaatgatgagcacttttaaagttctgctatgtggcgcggtattatcccgtattgacgccgggcaagagcaactcggtcgcc gcatacactattctcagaatgacttggttgagtactcaccagtcacagaaaagcatcttacggatggcatgacagtaagagaattatgcagtgctgccataaccatgagtgataacactgcggc caacttacttctgacaacgatcggaggaccgaaggagctaaccgcttttttgcacaacatgggggatcatgtaactcgcttgatcgttgggaaccggagctgaatgaagccataccaaacg acgagcgtgacaccacgatgcctgtagcaatggcaacaacgttgcgcaaactattaactggcgaactacttactctagcttcccggcaacaattaatagactggatggaggcggataaagtt gcaggaccacttctgcgctcggcccttccggctggctggtttattgctgataaatctggagccggtgagcgtgggtctcgcggtatcattgcagcactggggccagatggtaagccctcccgt atcgtagttatctacacgacggggagtcaggcaactatggatgaacgaaatagacagatcgctgagataggtgcctcactgattaagcattggtaactgtcagaccaagtttactcatatatac tttagattgatttaaaacttcatttttaatttaaaaggatctaggtgaagatcctttttgataatctcatgaccaaaatcccttaacgtgagttttcgttccactgagcgtcagaccccgtagaaaaga tcaaaggatcttcttgagatcctttttttctgcgcgtaatctgctgcttgcaaacaaaaaaaccaccgctaccagcggtggtttgtttgccggatcaagagctaccaactctttttccgaaggtaact ggcttcagcagagcgcagataccaaatactgtccttctagtgtagccgtagttaggccaccacttcaagaactctgtagcaccgcctacatacctcgctctgctaatcctgttaccagtggctgct gccagtggcgataagtcgtgtcttaccgggttggactcaagacgatagttaccggataaggcgcagcggtcgggctgaacggggggttcgtgcacacagcccagcttggagcgaacgac ctacaccgaactgagatacctacagcgtgagctatgagaaagcgccacgcttcccgaagggagaaaggcggacaggtatccggtaagcggcagggtcggaacaggagagcgcacgag ggagcttccagggggaaacgcctggtatctttatagtcctgtcgggtttcgccacctctgacttgagcgtcgatttttgtgatgctcgtcaggggggcggagcctatggaaaaacgccagcaa cgcggcctttttacggttcctggccttttgctggccttttgctcacatgt 


\section{LIST OF FIGURES}

Figure 1.1: Jablonski energy diagram ...........................................................................................................................

Figure 1.2: Principle of STED nanoscopy ...........................................................................................................

Figure 2.1: Scheme of the custom-built dual-color far-red STED nanoscope for in vivo mouse imaging..15

Figure 2.2: Spectral ranges of the three detection channels...............................................................................18

Figure 2.3: Schematic of electronic control- and signal pathways ....................................................................19

Figure 2.4: Phase pattern displayed on the SLM ................................................................................................20

Figure 2.5: Flow chart of animal experiments ........................................................................................................21

Figure 2.6: Schematic of viral injection into the visual cortex of the left hemisphere.......................................23

Figure 2.7: Mouse head fixation ....................................................................................................................................25

Figure 2.8: Surgical preparation of the cranial window ..........................................................................................26

Figure 2.9: Schematic of fluorescent dye injection into the visual cortex of the left hemisphere................28

Figure 2.10: Normalized absorbance and emission spectra of selected fluorescent spheres .........................30

Figure 3.1: PSF measurements on $80 \mathrm{~nm}$ gold beads.........................................................................................38

Figure 3.2: Comparison of the image quality of $40 \mathrm{~nm}$ crimson beads by using different imaging

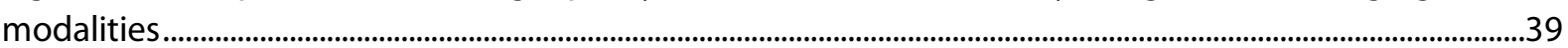

Figure 3.3: Confocal PSF estimation from images of $40 \mathrm{~nm}$ fluorescent beads ...............................................41

Figure 3.4: Images of $20 \mathrm{~nm}$ crimson beads with confocal and maximum STED resolution ..........................43

Figure 3.5: Resolution improvement of $20 \mathrm{~nm}$ crimson beads with increasing depletion laser power .....44

Figure 3.6: Resolution increase in STED nanoscopy of tubulin filaments in living Vero cells, labeled with

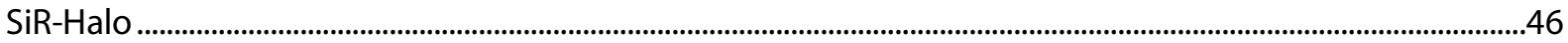

Figure 3.7: Specificity of the AAV-based expression of mNeptune2-labeled Actin-Chromobody in neurons of the visual cortex using the hSyn promotor .................................................................................................50

Figure 3.8: In vivo STED imaging of far-red fluorescent proteins in the visual cortex.......................................52

Figure 3.9: In vivo STED imaging of Lifeact-mCardinal in dendrites of pyramidal neurons of the visual

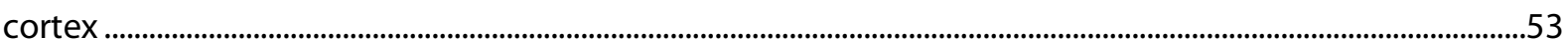

Figure 3.10: Specific and efficient in vivo labeling of Lifeact-Halo in neurons of the visual cortex using the

hSyn promotor .......................................................................................................................................................5

Figure 3.11: HaloTag-labeling of actin cytoskeletal organization in dendrites of the visual cortex in living

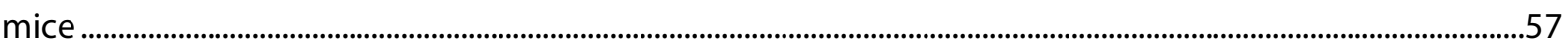

Figure 3.12: In vivo imaging of endogenous PSD-95 in the visual cortex.....................................................60

Figure 3.13: In vivo STED nanoscopy of endogenous PSD-95 in the molecular layer of the visual cortex 61

Figure 3.14: Gallery of round- or oval-shaped nanoscale organizations of PSD-95 in vivo...............................62

Figure 3.15: In vivo STED nanoscopy reveals an elliptical organization of PSD-95 ............................................63

Figure 3.16: Gallery of elongated nanoscale organizations of PSD-95 in vivo .....................................................64

Figure 3.17: Size analysis of the elongated organization of PSD-95...................................................................65

Figure 3.18: Gallery of the complex-shaped nanoscale organizations of PSD-95 in vivo ..................................66

Figure 3.19: Three-dimensional nanoscale organization of PSD-95 in vivo.........................................................67

Figure 3.20: Long-term time-lapse imaging reveals rearrangements of individual PSD-95 structures in a

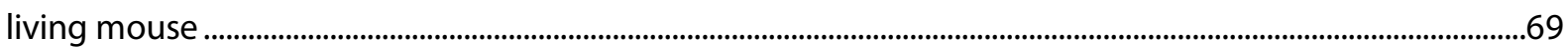

Figure 3.21: Short-term time-lapse imaging reveals rearrangements of individual PSD-95 structures in a

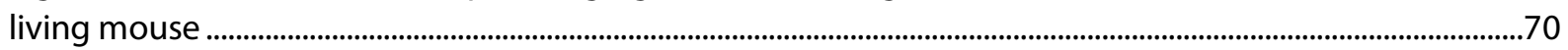

Figure A.1: Overview of the custom-built compact STED nanoscope................................................................90

Figure A.2: Final configuration of the STED nanoscope with the additional mechanical supports .............91

Figure A.3: PSF of gated and non-gated STED nanoscopy ……........................................................................92

Figure A.4: Calculated intensity distributions in $x y$ - and $y z$-direction for each excitation-detection

combination ..................................................................................................................................................93

Figure A.5: In vivo STED nanoscopy of endogenous PSD-95 in dendrites in the molecular layer of the visual cortex of a mouse.. 
Figure A.6: In vivo STED nanoscopy of endogenous PSD-95 in dendrites in the molecular layer of the visual cortex of a mouse.

Figure A.7: In vivo STED nanoscopy of endogenous PSD-95 in dendrites in the molecular layer of the visual cortex of a mouse

Figure A.8: In vivo STED nanoscopy of endogenous PSD-95 in dendrites in the molecular layer of the visual cortex of a mouse.

Figure A.9: In vivo STED nanoscopy of endogenous PSD-95 in dendrites in the molecular layer of the visual cortex of a mouse...

Figure A.10: In vivo STED nanoscopy of endogenous PSD-95 in dendrites in the molecular layer of the visual cortex of a mouse.

Figure A.11: In vivo STED nanoscopy of endogenous PSD-95 in dendrites in the molecular layer of the visual cortex of a mouse

Figure A.12: In vivo STED nanoscopy of endogenous PSD-95 in dendrites in the molecular layer of the visual cortex of a mouse. . .98

Figure A.13: In vivo STED nanoscopy of endogenous PSD-95 in dendrites in the molecular layer of the visual cortex of a mouse. 


\section{LIST OF TABLES}

Table 2.1: Adeno-associated viral vectors ........................................................................................................................22

Table 2.2: Fluorescent organic dyes.............................................................................................................................2

Table 2.3: Overview of selected fluorescent spheres ......................................................................................................29

Table 3.1: Characteristics of red to far-red fluorescent proteins..................................................................................48

Table 3.2: Characteristics of the used far-red fluorescent organic dyes .......................................................................54

Table A.1: Overview of image acquisition parameters .................................................................................................. 100 


\section{CONTRIBUTIONS}

This work was carried out in the Department of NanoBiophotonics at the Max Planck Institute for Biophysical Chemistry (MPI-BPC) in Göttingen, Germany. Stefan W. Hell initiated and supervised the project.

The first version of the STED nanoscope was designed and constructed by myself together with Johann Engelhardt and Joachim Fischer, both at the German Cancer Research Center (DKFZ) in Heidelberg, Germany. I carried out further developments of the setup, conducted the maintenance of alignment, and characterized the performance of the STED nanoscope.

Labeling strategies were developed and investigated by myself with assistance of Dirk Kamin. The in vivo STED imaging experiments were designed by myself together with Dirk Kamin. Mice surgery and preparation was executed by myself together with Dirk Kamin and Heinz Steffens. I performed in vivo STED imaging and carried out all data analysis. Fixed brain slices were prepared and imaged by Jasmine Hubrich. The PSD-95-HaloTag mouse line was provided by Seth G. N. Grant (Centre for Clinical Brain Sciences, Edinburgh University, Edinburgh, United Kingdom) to initiate a mouse colony at the MPI-BPC. Mice breeding was conducted by myself together with Dirk Kamin. Care of the mice was carried out by the animal facility of the MPI-BPC. Tanja Gilat and Jasmine Hubrich genotyped the transgenic mice. 


\section{ACKNOWLEDGMENTS}

This dissertation would not have been possible without the inspiration and support of a number of wonderful and talented individuals. To all of them, I would like to express my acknowledgement and appreciation for being part of this journey.

Foremost, I owe my deepest sense of gratitude to my advisor Stefan W. Hell for giving me the opportunity to work on this fascinating and challenging project. I thank him for his scientific guidance and for providing an excellent atmosphere for doing research. My $\mathrm{PhD}$ has been a thrilling experience in an inspiring working environment. Thank you so much!

Besides my advisor, I would like to thank my thesis committee members: Detlev Schild and Nils Brose for their interest in my project as well as their advices given on my work. I am also indebted to Sarah Köster, Michael Hörner, and Alexander Egner willing to participate in my examination board.

I would like to express my sincere thanks to Dirk Kamin and Nicolai T. Urban for being excellent mentors. You both helped me a lot getting the project started by sharing your broad knowledge in the field of super-resolution microscopy and neuroscience. Many comments and discussions contributed to the success of this work. I am glad that I could count on both of you during the last 3.5 years.

Most of the results described in this thesis would not have been achieved without the support of my project members Dirk Kamin and Heinz Steffens. Both shared the same passion and enthusiasm to push the limits of in vivo STED nanoscopy even further. While sitting together in a dark lab for hours, they provided the best possible company. I am especially grateful to Heinz for sharing his mice preparation expertise willingly and being so patient during the surgical training.

My sincere thanks also goes to Johann Engelhardt and Joachim Fischer, on whose support the STED setup was constructed within a short time. I really appreciate their scientific advices and fundamental knowledge in the field of super-resolution microscopy. I have learned a lot during my brief stay at the German Cancer Research Center (DKFZ) in Heidelberg and would like to thank the Optical Nanoscopy group for creating such a nice atmosphere. A special thanks also belongs to Johann for his software support, which he willingly provided, while sometimes at very short notice.

I also want to express my gratitude to the collaboration partners Seth G. N. Grant and Maximilian Kratschke from the University of Edinburgh for providing the transgenic PSD-95-HaloTag mouse and for their commitment to the success of imaging PSD-95 in vivo.

I greatly appreciate the support by Elisa D'Este for sharing her knowledge in the field of neuroscience and super-resolution microscopy. Thank you also for your energy, your addictive enthusiasm for science, for the great discussions, and all kinds of help and advice you gave me.

Special thanks go also to Dirk Kamin, Jan Keller, Elisa D'Este, Jaydev Jethwa, Nicolai T. Urban, Joachim Fischer, Johann Engelhardt, Heinz Steffens, Jasmine Hubrich, Mackenzie Lee, Sandro Kock, Beate Koch, and Anna Heye for critically reading parts of the manuscript and their helpful comments. 
I am indebted to many of my present and former colleagues of the NanoBiophotonics department for their assistance and providing a nice working atmosphere. I especially thank

Carola Gregor, Tanja Gilat, and Jasmine Hubrich for viral cloning, cell culture, and mouse genotyping

Alexey Butkevich and Grazvydas Lukinavičius for providing the organic dyes

Jaydev Jethwa for finding a solution for absolutely everything

Rainer Pick for technical and mechanical support

Marco Roose for software and hardware support

Jan Keller for his kind help with programming on the analysis of the setup performance

Katrin I. Willig, Sven-Christian Sidenstein, Klaus Gwosch, and Fabian Göttfert, who have shared their expertise in planning, adjusting and optimizing optical microscopes

Ulrike Böhm for scientific advices and many insightful conversations

Thanks to Ulrike Teichmann, Sarah Kimmina, Kirsten Kiel, and other colleagues from the MPIBPC animal facility for supporting the animal studies with their knowledge and taking good care of the mice.

I would also like to thank the colleagues from the MPI-BPC mechanical, electronics, and optics workshops, who have been kind enough to advise and help in their respective roles.

Many thanks go to my friends Maria K., Stefanie K., Isabelle J., Philipp A., Dirk K., Sebastian S., Dirk M., Tanja G., Jasmine H., Christian B., and Timo K. from the NanoBiophotonics department for all the great moments we have shared. All of you have been exceptionally supportive and helped me keeping my focus and motivation. I am particularly thankful to Stefanie K. and Isabelle J. for being such ambitious fitness partners.

I am also deeply grateful to my family for their love, support, and sacrifices through my life and especially during the last 3.5 years. Finally, I am tremendously thankful and delighted that Sandro shares his love and life with me. Thank you very much for believing in me. 